\title{
Dissecting the molecular function of the ubiquitin-like Atg8 during autophagosome biogenesis in S. cerevisiae
}

\author{
Doctoral Thesis \\ In partial fulfillment of the requirements for the degree \\ "Doctor rerum naturalium (Dr. rer. nat.)" \\ in the Molecular Medicine Study Program \\ at the Georg-August University Goettingen
}

submitted by

Anne Lisa Mitter

born in Hanover

Goettingen, 2017 
Member of the Thesis Committee and Supervisor:

Prof. Dr. Michael Thumm

Department of Cellular Biochemistry

Center for Biochemistry and Molecular Cell Biology

University Medical Center Goettingen

Humboldtallee 23

37073 Goettingen

Second member of the Thesis Committee:

Prof. Dr. Silvio O. Rizzoli

Department of Neuro- and Sensory Physiology

University Medical Center Goettingen

Humboldtallee 23

37073 Goettingen

Third member of the Thesis Committee:

Prof. Dr. Stefanie Pöggeler

Institute of Microbiology and Genetics

Department of Genetics of Eukaryotic Microorganisms

Georg-August University of Goettingen

Grisebachstraße 8

37077 Goettingen

Date of oral examination: 
Affidavit:

Here I declare, that my doctoral thesis entitled "Dissecting the molecular function of the ubiquitin-like Atg8 during autophagosome biogenesis in S. cerevisiae" has been written independently and with no other sources and aids than quoted.

Anne Lisa Mitter Goettingen, December 2017 


\section{Content}

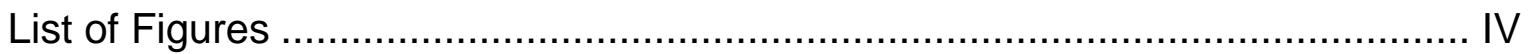

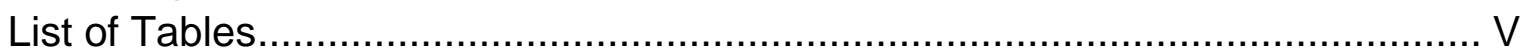

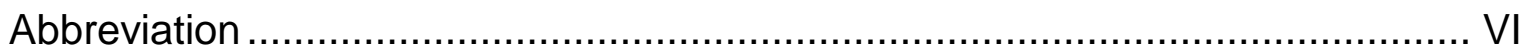

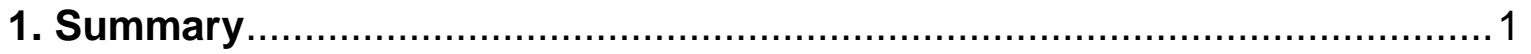

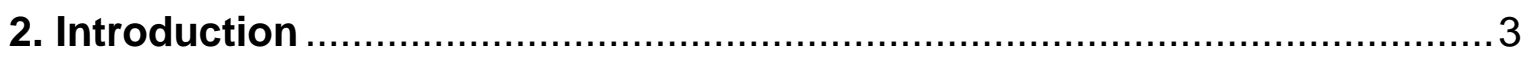

2.1 The model organism Saccharomyces cerevisiae ..................................... 3

2.2 Regulation of protein and vesicle transport in S. cerevisiae ....................... 4

2.2.1 Ypt/Rab GTPases are regulators of protein traffic ............................. 6

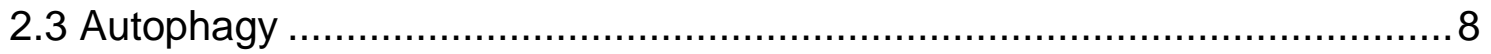

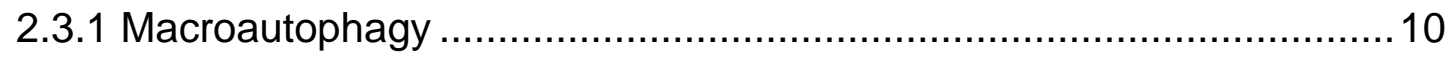

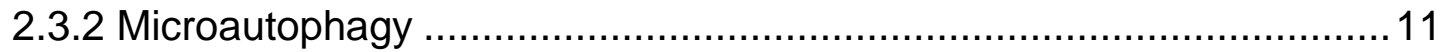

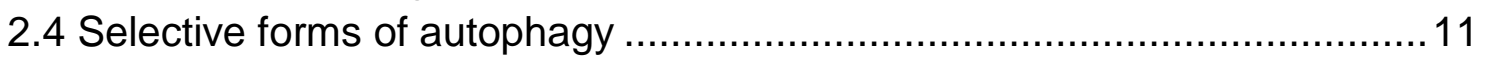

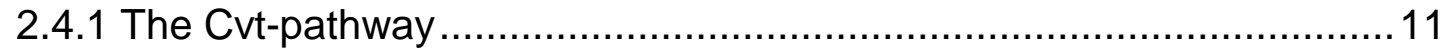

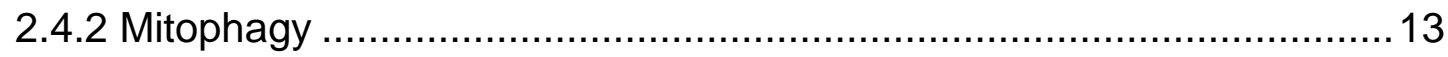

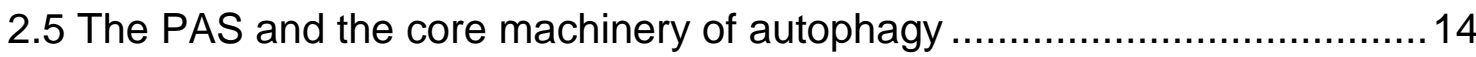

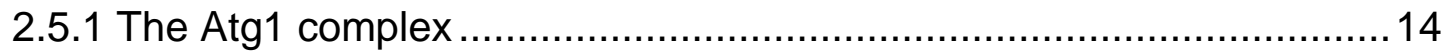

2.5.2 Phosphatidylinositol 3-kinase complex ………............................. 17

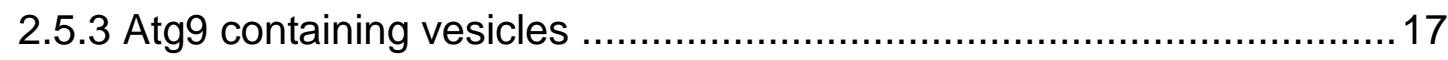

2.5.4 COPII mediated vesicle formation.................................................. 19

2.5.5 Two ubiquitin-like protein conjugation complexes..............................2

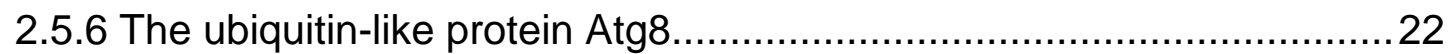

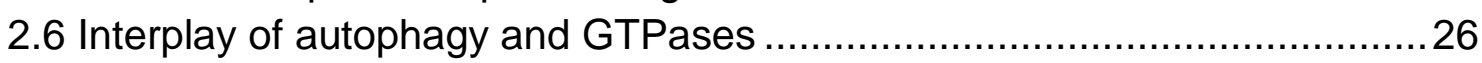

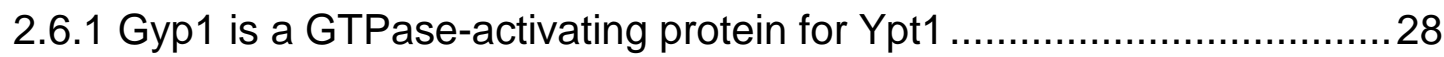

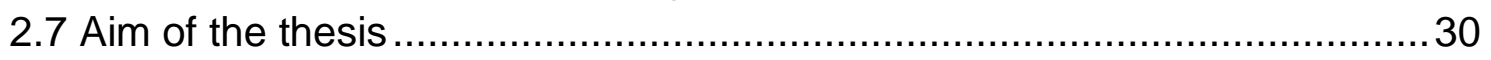

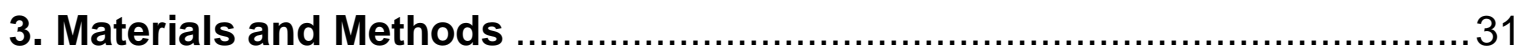

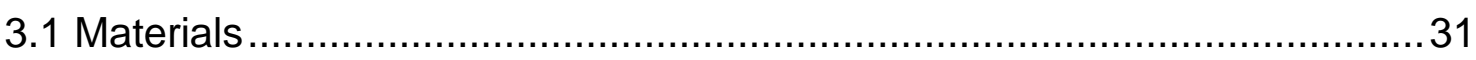

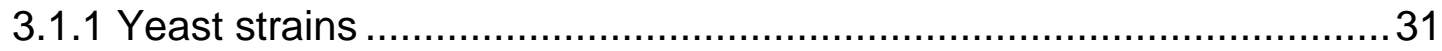

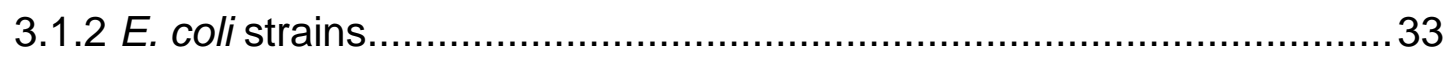

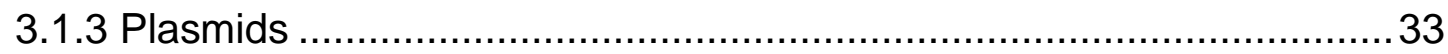

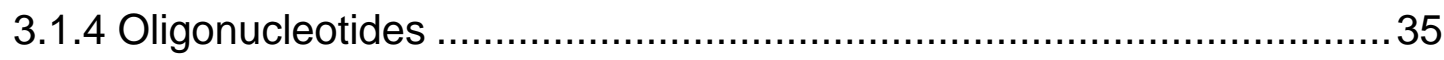

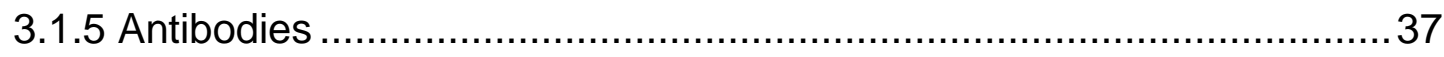

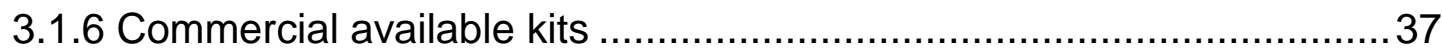

3.1.7 Chemicals, supplements, enzymes and protein purification systems .. 38

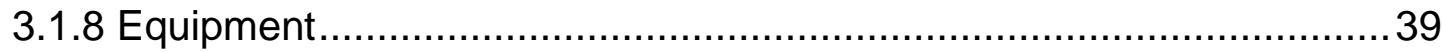

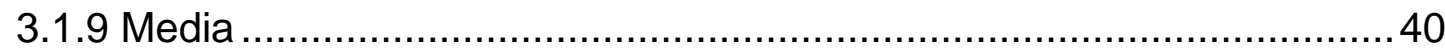

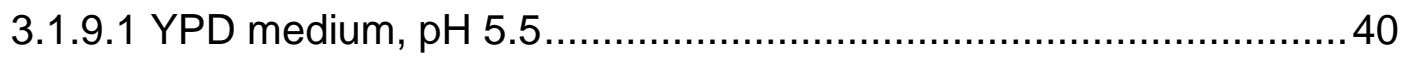

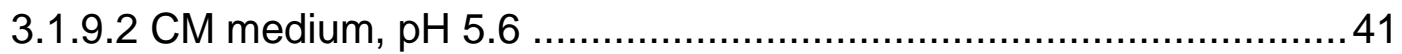

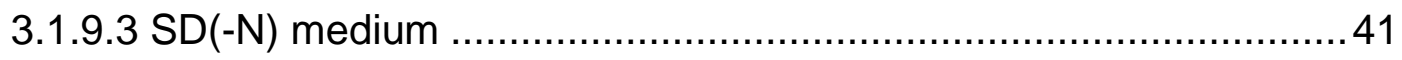

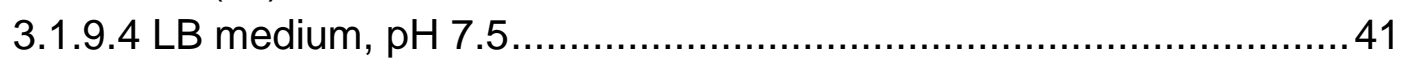




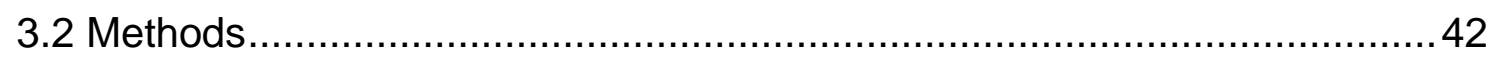

3.2.1 Cultivation and storage of $S$. cerevisiae .................................... 42

3.2.2 Cultivation and storage of E. coli........................................... 42

3.2.3 Molecular biological methods.............................................. 43

3.2.3.1 Determination of cell density ......................................... 43

3.2.3.2 Preparation of electrocompetent $E$. coli cells............................43 43

3.2.3.3 Determination of the DNA concentration ............................... 43

3.2.3.4 Chromosomal DNA extraction from yeast cells ........................ 43

3.2.3.5 Polymerase chain reaction (PCR) ....................................... 44

3.2.3.6 DNA agarose gel electrophoresis ..................................... 44

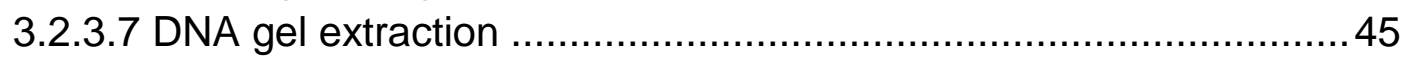

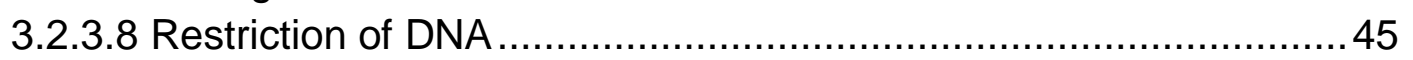

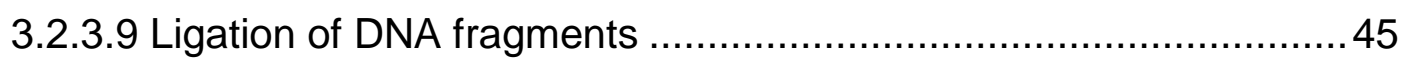

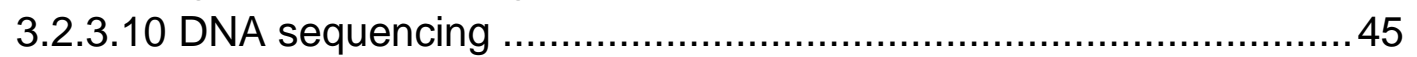

3.2.3.11 Molecular cloning ...................................................... 45

3.2.3.12 Site-directed mutagenesis of plasmids .............................. 46

3.2.3.13 Plasmid purification form $E$. coli ..................................... 46

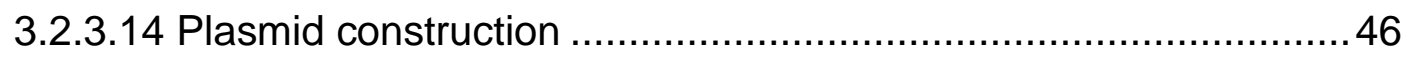

3.2.3.15 Yeast cell transformation with high efficiency ......................... 47

3.2.3.16 "Quick and dirty" transformation ...................................... 47

3.2.3.17 Construction of knock out strains .................................... 48

3.2.4 Direct fluorescence microscopy ........................................... 48

3.2.5 Split-ubiquitin assay ................................................................ 49

3.2.6 Monitoring of pApe1 maturation .......................................... 51

3.2.7 Induction and monitoring of mitophagy .................................... 51

3.2.8 Biochemical methods ......................................................... 51

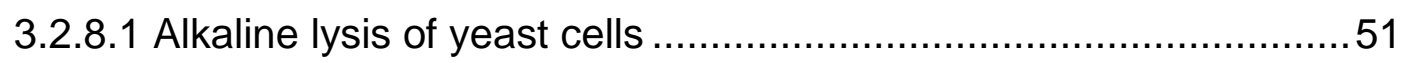

3.2.8.2 SDS-Polyacrylamide-Gel-Electrophoresis (SDS-PAGE) ............. 52

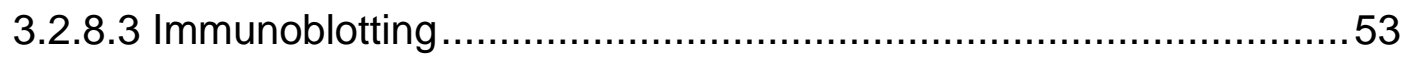

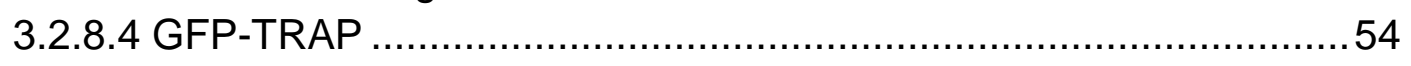

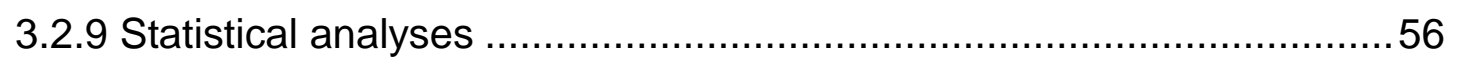

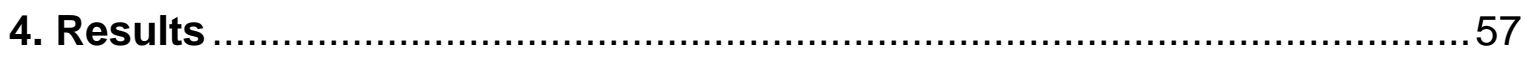

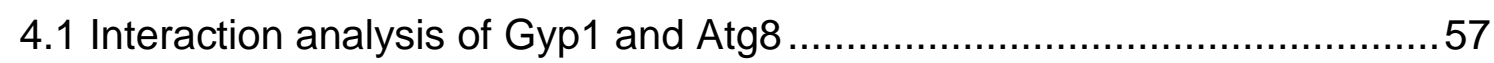

4.1.2 Atg8 preferentially interacts with Gyp1 ...................................... 57

4.1.3 Gyp1 interacts with the AIM binding sites of Atg8 .............................58

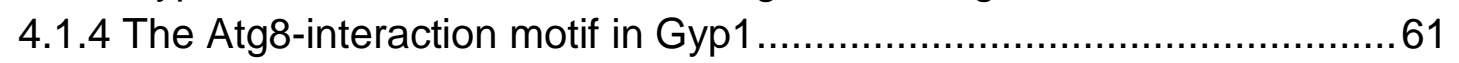

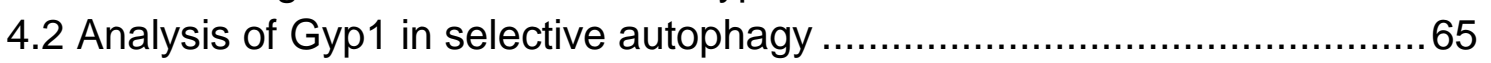

4.2.1 Gyp1 is needed for proper proceeding of the Cvt-pathway................65

4.2.2 Gyp1 is important for proper proceeding of post-log Mitophagy...........69

4.3 Gyp1 and Atg8 co-localize using fluorescence microscopy......................71

4.4 Analysis of the Cvt-pathway using fluorescence microscopy ...................73 
4.4.1 Localization of pApe1 and Atg11 at the PAS does not require Gyp1 ...73

4.4.2 Gyp1 plays a role in disassembly of the Ypt1-Atg1 complex .............. 75

4.4.3 Gyp1 is important for proper recruitment of Atg14 to the PAS .............78

4.4.4 Gyp1 is needed for proper localization of Atg8 at the PAS .................80 80

4.5 Analysis of Atg8 binding to specific cargo receptors............................ 83

4.5.1 Interaction of Atg8 and the cargo receptor for mitophagy Atg32 depends

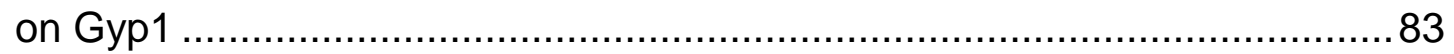

4.5.2 Binding of Atg8 to the cargo receptor Atg19 is reduced upon GYP1

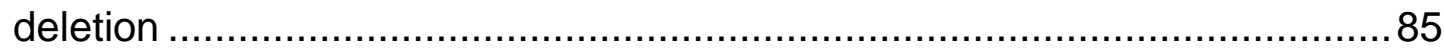

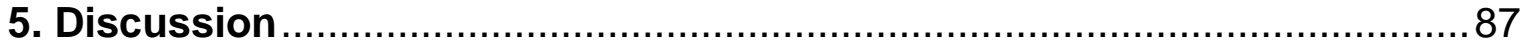

5.1 Identification of Gyp1 as an Atg8 interaction partner .............................. 88

5.2 Gyp1 contributes to selective forms of autophagy ............................... 92

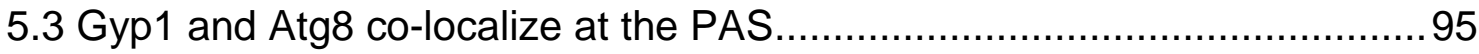

5.4 The role of Gyp1 in the Cvt-pathway ......................................... 97

5.4.1 The Cvt-complex assembles properly in the absence of Gyp1 ...........99 97

5.4.2 Gyp1 leads to efficient dissociation of the Ypt1-Atg1 complex.............99

5.4.3 Disassembly of the Ypt1-Atg1 complex is a prerequisite for Atg14 recruitment................................................................................. 100

5.4.4. GAP activity of Gyp1 is needed for recruitment of Atg8 to the PAS...101

5.5. Gyp1 is involved in cargo recognition by Atg8 ................................... 104

5.6 Gyp1 has a dual function during selective autophagy ....................... 106

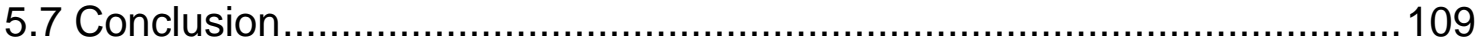

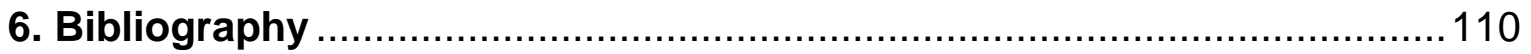

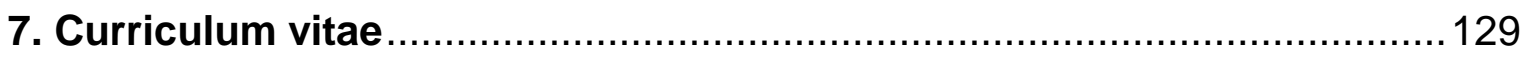




\section{List of Figures}

Figure 2.1: $\quad$ Life cycle of Saccharomyces cerevisiae 3

Figure 2.2: $\quad$ The cycling of Rabs 7

Figure 2.3: $\quad$ Types of autophagy (Yen and Klionsky, 2008) 9

Figure 2.4: The Cvt-pathway and unselective autophagy in yeast 12

Figure 2.5: Mitophagy in yeast (modified after Kanki et al., 2015) 13

Figure 2.6: $\quad$ The Atg1 kinase complex 15

Figure 2.7: Cycling of Atg9 containing vesicles (Yamamoto et al. 18 2012)

Figure 2.8: Role of COPII vesicles in autophagosome biogenesis 20 (Davies and Ferro-Novick, 2015)

Figure 2.9: The ubiquitin-like conjugation of Atg8 (modified from 21 Nakatogawa et al., 2009)

Figure 2.10: Atg8 interacts via AIMs and is initially located at both 25 sides of the forming phagophore

Figure 3.1: The split-ubiquitin system 50

Figure 3.2: $\quad$ Immunoblot setting (adapted from Mini Trans-Blot ${ }^{\circledR} \quad 53$ Electrophoretic Transfer Cell Instruction Manual)

Figure 3.3: $\quad$ GFP-TRAP procedure 54

Figure 4.1: Atg8 interacts preferentially with Gyp1 58

Figure 4.2: $\quad$ Binding of Gyp1 and Atg8 is reduced after mutating the $\quad 59$

Figure 4.3: $\quad$ Putative AIMs in Gyp1 62

Figure 4.4: Binding of Gyp1_AIM1 to Atg8 is reduced in vivo 64

Figure 4.5: $\quad$ pApe1 processing is impaired upon GYP1 deletion under $\quad 66$ nutrient-rich conditions

Figure 4.6: Gyp1 plays a role in proceeding of post-log mitophagy $\quad 70$

Figure 4.7: $\quad$ GFP-Atg8 and Gyp1 co-localize $\quad 72$

Figure 4.8: $\quad$ Analysis of the localization of Ape1-RFP and Cherry- $\quad 74$ Atg11 upon deletion of the GYP genes

Figure 4.9: $\quad$ Analysis of Atg1-GFP in the absence of Gyp1 77

Figure 4.10: Atg14-GFP localization is affected upon GYP1 deletion 78

Figure 4.11: Ypt1-Atg1 complex disassembly is a prerequisite for $\quad 79$

Figure 4.12: Proper Atg8 recruitment requires the Gyp proteins 80

Figure 4.13: The GAP activity of Gyp1 and its interaction with Atg8 are 82 needed for proper Atg8 localization at the PAS

Figure 4.14: The interaction of Atg8 and Atg32 is reduced upon 84 deletion of GYP1

Figure 4.15: Binding of Atg8 to Atg19 in the absence of Gyp1 analyzed 85 by the split-ubiquitin assay.

Figure 5.1: Gyp1 has dual functions in autophagy 107 


\section{List of Tables}

Table 3.1: $\quad$ Yeast strains used in this study 31

Table 3.2: E. coli strains used in this study 33

Table 3.3: $\quad$ Plasmids used in this study 33

Table 3.4: Oligonucleotides used in this study 35

Table 3.5: $\quad$ Antibodies used in this study 37

Table 3.6: Commercial available kits used in this study 37

Table 3.7: Chemicals, supplements, enzymes and protein 38 purification systems used in this study

Table 3.8: $\quad$ Equipment used in this study 39

Table 3.9: $\quad$ Filter sets for live cell imaging 49

Table 3.10: Contents of SDS Polyacrylamid gel used in a Bio-Rad 52

Table 3.11: $\quad$ GFP-TRAP buffers $\quad 55$

Table 3.12: $\quad$ GFP-TRAP conditions for different experimental setups $\quad 55$ 


\section{Abbreviation}

\begin{tabular}{|c|c|}
\hline - & without \\
\hline${ }^{\circ} \mathrm{C}$ & Degree in Celsius \\
\hline A & Ampere \\
\hline ade & Adenine \\
\hline AIM & Atg8-interacting motif \\
\hline Ams1 & Alpha-mannosidase 1 \\
\hline Arg & Arginine \\
\hline Ape1 & Aminopeptidase 1 \\
\hline APS & Ammonium persulfate \\
\hline $\operatorname{Arg}$ & Arginine \\
\hline Atg & Autophagy-related \\
\hline bp & base pair \\
\hline $\mathrm{c}$ & chromosomal \\
\hline $\mathrm{CM}$ & Complete minimal medium \\
\hline COP & Coat protein \\
\hline CPY & Carboxypeptidase $Y$ \\
\hline Cub & C-terminal part of ubiquitin \\
\hline Cvt & Cytoplasm-to-vacuole targeting \\
\hline DNA & Desoxyribonucleic acid \\
\hline E. coli & Escherichia coli \\
\hline EDTA & Ethylenediamintetraacetate-disodium salt \\
\hline ER & Endoplasmic reticulum \\
\hline FOA & 5-Fluoroorotic acid \\
\hline g & gramm \\
\hline g & gravity \\
\hline GAP & GTPase activating enzyme \\
\hline GABARAB & Gamma-aminobutyric acid receptor-associated protein \\
\hline GDI & Guanine nucleotide dissociation inhibitors \\
\hline GDP & Guanosine diphosphate \\
\hline GEF & Guanine nucleotide exchange factor \\
\hline GFP & Green fluorescent protein \\
\hline Gln & Glutamine \\
\hline Glu & Glutamic acid \\
\hline Gly & Glycine \\
\hline GST & Gluthathione-S-transferase \\
\hline GTP & Guanosine-5'-triphosphate \\
\hline Gyp1 & GTPase-activating protein for Ypt1 \\
\hline $\mathrm{h}$ & hour(s) \\
\hline $\mathrm{HA}$ & Human influenza hemagglutinin \\
\hline His & Histidine \\
\hline IM & Inner membrane \\
\hline KAN & Kanamycin \\
\hline kDA & kilodalton \\
\hline I & liter \\
\hline LE & Lysogney broth \\
\hline
\end{tabular}




$\begin{array}{ll}\text { Leu } & \text { Leucine } \\ \text { LIR } & \text { LC3-interacting region } \\ \text { Lys } & \text { Lysine } \\ \text { M } & \text { Molar } \\ \mathrm{m} & \text { mature } \\ \mathrm{m} & \text { meter } \\ \mathrm{m} & \text { milli } \\ \text { Met } & \text { Methionine } \\ \text { min } & \text { minute } \\ \mathrm{N} & \text { Nitrogen } \\ \mathrm{n} & \text { nano } \\ \text { NatNT2 } & \text { Nourseotricine } \\ \text { NHD } & \text { N-terminal helical domain } \\ \text { Nub } & \text { N-terminal part of ubiquitin } \\ \text { OD } 600 & \text { Optical density (600 nm) } \\ \text { OM } & \text { Outer membrane } \\ \text { PAS } & \text { Pre-autophagosomal structure / pre-autophagosomal } \\ \text { PE } & \text { assemby site } \\ \text { pH } & \text { Phosphatidyethanolamine } \\ \text { Phe } & \text { potential of hydrogen } \\ \text { PI3P } & \text { Phenylalanine } \\ \text { Ptdlns } & \text { Phosphatidylinositol 3-phosphate } \\ \text { PMSF } & \text { Phosphatidylinositol } \\ \text { pr/p } & \text { Phenylmethylsulfonylfluoride } \\ \text { Rab } & \text { precursor } \\ \text { RFP } & \text { Ras genes from rat brain } \\ \text { rpm } & \text { Red fluorescent protein } \\ \text { RT } & \text { revolutions per minute } \\ \text { S } & \text { Room temperature } \\ \text { S. cerevisiae } & \text { second } \\ \text { SD } & \text { Saccharomyces cerevisiae } \\ \text { SDS } & \text { Synthetic defined } \\ \text { TBC domain } & \text { Sodium dodecyl sulfate } \\ \text { TCA } & \text { Tre-2, Bub2, Cdc16 domain } \\ \text { TOR } & \text { Trichloroacetic acid } \\ \text { Trp } & \text { Target of rapamycin } \\ \text { Ura } & \text { Tryptophane } \\ \text { v/v } & \text { Uracile } \\ \text { V } & \text { volume per volume } \\ \text { w/v } & \text { Volt } \\ \text { WT } & \text { weight per volume } \\ \text { YPD } & \text { Wild type } \\ \mu & \text { Yeast peptone dextrose } \\ \text { B-ME } & \text { Beta-mercaptoethanol } \\ & \end{array}$




\section{Summary}

Autophagy is a degradative pathway that is conserved from yeast to mammals. Marcoautophagy is characterized by the formation of a unique double-membrane layered vesicle, the so-called autophagosome. Proteins and organelles are engulfed by the autophagosome and transported to the degradative compartment, which is the vacuole in yeast and plants or the lysosome in mammals. At the degradative compartment, the outer membrane of the autophagosome fuses with the membrane. Then the mono-layered inner vesicle, the autophagic body, is released and its contents are degraded. Autophagy can be a selective process that targets specific proteins or organelles to the degradative compartment by using specific cargo receptors. During unselective autophagy proportions of the cytoplasm are enclosed in a non-specific manner and degraded. The autophagic process is important to maintain cell viability especially under stress conditions and to adapt to changing environments. Degraded components can finally be reused to maintain nutrient supply for the cell. Autophagy plays a role in various processes in the cell like aging, cell homeostasis and host defense. Malfunction of the autophagic process is associated with various diseases like cancer or neurodegeneration. Thus, autophagy has to be tightly controlled. One element of autophagy that needs to be tigthly controlled is the membrane traffic to the PAS, which is required for the formation of the autophagosome.

Key regulators of intracellular membrane traffic are the Ypt/Rab GTPases (Ypts in yeast and Rabs in mammals), which mediate directed and specified intracellular vesicle transport through the cell to their final destination. Ypt/Rab GTPases are localized to different compartments and contribute to membrane identity. To fulfill their duties in the cell, Ypt/Rab GTPases cycle between a GTP-bound form, which activates the protein and allows binding to its effectors, and a GDP-bound form. The transition of both forms requires GEFs (guanine-nucleotide exchange factors) and GAPs (GTPase-activating proteins). Ypt1 is a yeast Rab GTPase that mediates vesicle transport and also plays a role in autophagy. Ypt1 interacts with Atg proteins and contributes to phagophore formation.

Previous to this study, Gyp1 has been identified as a potential interaction partner of Atg8 by mass spectrometry. Atg8 plays a role in elongation and closure of the phagophore and targets cargos to the autophagosome during selective forms of 
autophagy. Gyp1 is a GAP and known to negatively regulate the Rab GTPase Ypt1 by stimulating its GTPase activity. Three different GAPs, Gyp1, Gyp5 and Gyp8 have been shown to negatively regulate the Rab GTPase Ypt1 by stimulating its GTPase activity in vivo.

This study shows that Gyp1 affects the Cvt-pathway, demonstrated by analysis of pApe1 maturation, proper proceeding of post-log mitophagy and might also play a role during Atg8-dependent recognition of other targets. Two functions of Gyp1 are proposed. First, Gyp1 seems to regulate the efficient dissociation of the Ypt1Atg1 complex during initial steps of phagophore assembly. In the absence of Gyp1, this complex is enriched and the recruitment of downstream proteins is impaired, as shown for Atg14 in this study. This indicates that dissociation of Ypt1 and Atg1 is a prerequisite for recruitment of Atg proteins and proceeding of selective autophagy. For this process, the GAP activity of Gyp1 is needed. Gyp5 and Gyp8 might compensate the loss of Gyp1 as additional deletion of the two other Ypt1 GAPs leads to a more severe impairment of the Cvt-pathway. Singledeletion of both proteins causes no defect. Thus, Gyp1 might function as the main GAP during the Cvt-pathway.

The second proposed function of Gyp1 was detected in later steps of selective autophagy. Here, Gyp1 interacts in an AIM-dependent manner with Atg8. In the absence of Gyp1, the function of Atg8 is impaired, which was demonstrated in this study with the mitophagy receptor Atg32. Upon deletion of GYP1, Atg8 and Atg32 show a reduced binding, which indicates a role of Gyp1 during cargo recognition. The analysis of Gyp1 mutants revealed that this function is AIM-dependent but GAP activity independent.

Thus, this study proposes a dual role for Gyp1 during selective autophagy. First, it leads to the efficient dissociation of the Ypt1-Atg1 complex and later Gyp1 interacts with Atg8, which seems to be important for proper binding of Atg8 to selective cargo receptors. 


\section{Introduction}

\subsection{The model organism Saccharomyces cerevisiae}

The budding yeast Saccharomyces cerevisiae belongs to the family of the Sacchromycetae and is one of the best-studied eukaryotic model organisms. The unicellular organism has a round to oval shape and a size of about 5 to $10 \mu \mathrm{m}$ in diameter. There are two mating types, Mat a and $\alpha$, and the cells can either be haploid or diploid (Fig. 2.1). A diploid cell can undergo meiotic division, leading to sporulation. This occurs mostly upon nutrient limitation. During sporulation, an ascus containing four haploid spores, two of each mating type, is formed. Two haploid cells with different mating types can form a diploid cell. By formation of a bud, S. cerevisiae can also undergo mitotic division (Fig. 2.1). In a nutrient-rich environment, S. cerevisiae is able to double in about $90 \mathrm{~min}$ through mitotic division (Duina et al., 2014).

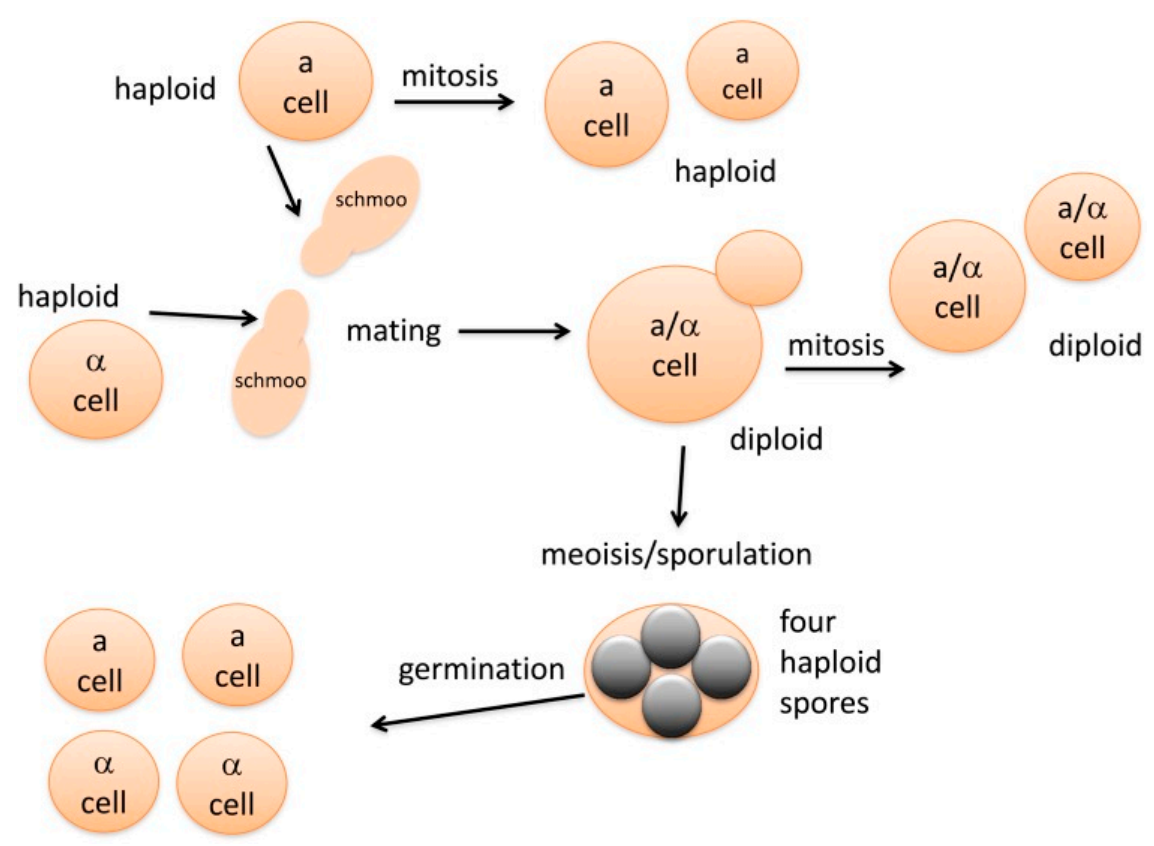

Figure 2.1: Life cycle of Saccharomyces cerevisiae

The two mating types Mat a and Mat $\alpha$ are haploid and bud by mitotic division. By mating, they generate diploid cells. Meiosis/sporulation leads to the formation of four haploid spores within the mother cell (Duina et al., 2014).

During the last decades, a variety of methods have been developed that allow to easily modify a gene or target allele with high precision (Longtine et al., 1998, Janke et al., 2004, Wach et al., 1997, Knop et al., 1999, Goffeau et al., 1996). 
Genes can be deleted or introduced by either directly editing the yeast genome or by utilizing plasmids. The full sequence of the genome is readily available. The genome consists of 16 chromosomes that comprise about 6000 genes. Shortly after publication of the complete yeast genome sequence, a set of deletions strains was established (Winzeler et al. 1998; Giaever et al., 2002). Today, a collection of knockout strains can be purchased from Euroscarf, American Type Culture Collection and Invitrogen (Scherens and Goffeau, 2004). Many of the S. cerevisiae genes are highly conserved even when compared to human homologs. Thus, findings obtained from the budding yeast can be highly relevant for research in higher eukaryotic cells. Homologs of many important genes in human biology had first been discovered and studied in yeast, making yeast an important and amenable model organism for biochemical and genetic research. In this regard, research areas like cancer and Parkinson's disease profit from this simple model organism (Billant et al., 2017; Tenreiro et al., 2017).

\subsection{Regulation of protein and vesicle transport in S. cerevisiae}

S. cerevisiae is a eukaryotic organism and has various membrane-enclosed compartments with specific functions. The transport of specialized vesicles is important for cargo trafficking between these different compartments. To maintain the functional organization in a yeast cell, this transport needs to be tightly controlled (Feyder et al., 2015). Vesicle enclosed proteins need to be transported to the acceptor compartment in a directed manner. Therefore, vesicles are selectively packed and recognized by their target membranes. The Golgi apparatus is responsible for sorting the synthesized proteins according to their transport specifications to the plasma membrane, to the external membrane for secretion and to the degradative compartment (Feyder et al., 2015). Some proteins can be transported directly through membrane contact sites between the Golgi and different organelles, while others need to be packed into vesicles for transport.

The synthesis of proteins for the secretory pathway is performed on the endoplasmic reticulum (ER). The ER is an organelle that forms tube-like structures and membrane enclosed sacs, building an often interconnected network. The smooth ER functions in lipid metabolism, detoxification and steroid 
hormone production. The rough ER is associated with ribosomes, which are responsible for protein synthesis (Schwarz and Blower, 2016). During their synthesis on the ER, proteins are translocated across the ER membrane while the polypeptide is still growing. From the ER, the proteins are transported to their destination in the cell (Lee et al., 2004). From the ER, COPII (coat protein complex II) vesicles target newly synthesized proteins to the Golgi apparatus and COPI vesicles recycle proteins back to the ER (Lee et al., 2004; Duden, 2013).

From the Golgi, proteins are transported e.g. to the plasma membrane via the secretory pathway or to endosomes from where they can be further transported to the vacuole. The vacuolar targeting pathway is best studied for the transport of carboxypeptidase $Y$ (CPY). CPY is transported from the Golgi to its final destination, the vacuole, through its specific membrane receptor Vps10. Vps10 targets CPY-containing vesicles to the endosome. Here, CPY-containing vesicles fuse with the endosomal compartment, Vps10 dissociates and is recycled to the Golgi. Fusion of the CPY-containing vesicle with the vacuole releases CPY, and it can be processed into its active form (Bowers and Stevens, 2005). Defects in protein targeting to the vacuole have been studied by screening mutants for their misdirected secretion of CPY into the extracellular medium instead of its correct targeting to the vacuole (Bonangelino et al., 2002).

The multivesicular body (MVB) pathway targets membrane proteins to the vacuole for degradation. Most of the proteins that are degraded by this pathway are monoubiquitinated. Ubiquitination, i.e. the addition of ubiquitin to a lysine residue of a target protein, serves as an entry signal for this pathway. Ubiquitin is recognized by the ESCRT (endosomal sorting complex required for transport) machinery, which mediates proper sorting of the target proteins into vesicles (Babst, 2011; Urbé, 2005; Katzmann et al., 2001). The MVB pathway is supposed to sense nutrient limitations and to secure cell survival under starvation together with autophagy (Müller et al., 2015).

The transport of proteins to the different organelles is tightly controlled. A class of proteins responsible for the control of protein traffic are the Rab (Ras genes from rat brain) proteins. Rab proteins are the largest family in the Ras-like GTPase 
superfamily. Their counterparts in yeast are the Ypt (Yeast protein transport) proteins (Segev, 2001; Lipatova et al., 2015).

\subsubsection{Ypt/Rab GTPases are regulators of protein traffic}

Ypt/Rab GTPases (guanosine triphosphatases) are known as master regulators of intracellular membrane traffic. GTPases are small proteins with a size of approximately 200 amino acids. They have been found in all eukaryotes where they regulate intracellular membrane trafficking pathways (Li and Segev, 2013). The GTPases localize to different compartments in a GTP-dependent manner, to contribute to membrane identity (Pfeffer et al., 2013). By recruiting effector proteins to defined membranes, Ypt/Rab GTPases facilitate a specified and directed transport of vesicles through the cell. Dysfunction of these proteins leads to a variety of diseases, ranging from rare hereditary diseases to mental retardation and infectious diseases (Seabra et al., 2002).

Ypt/Rab GTPases can act as molecular switches by cycling between a GTP- and a GDP-bound form (Fig. 2.2A). They can be activated by guanine-nucleotide exchange factors (GEFs) and inactivated by GTPase-activating proteins (GAPS). GAPs trigger the slow intrinsic GTP hydrolysis rate of their target GTPases. A GEF activates the GTPase protein by stimulating the release of guanosine diphosphate (GDP) to allow binding of guanosine triphosphate (GTP) (Fig. 2.2B), enabling the activated GTPase to bind to its effector protein (Lipatova et al., 2016).

Proteins that interact specifically with the GTP-bound form of the Ypt/Rab GTPase are defined as Rab effectors (Zhen and Stenmark, 2015) and a broad variety of proteins can function as such an effector like kinases, phosphatases, tethering factors etc. (Gillingham et al., 2014). Ypt/Rab GTPases exist in two pools: soluble and membrane-bound. By geranylgeranylation (mediated by Rab geranylgeranyl transferases), Ypt/Rab GTPases are associated to their target membranes (Leung et al., 2006). 
A

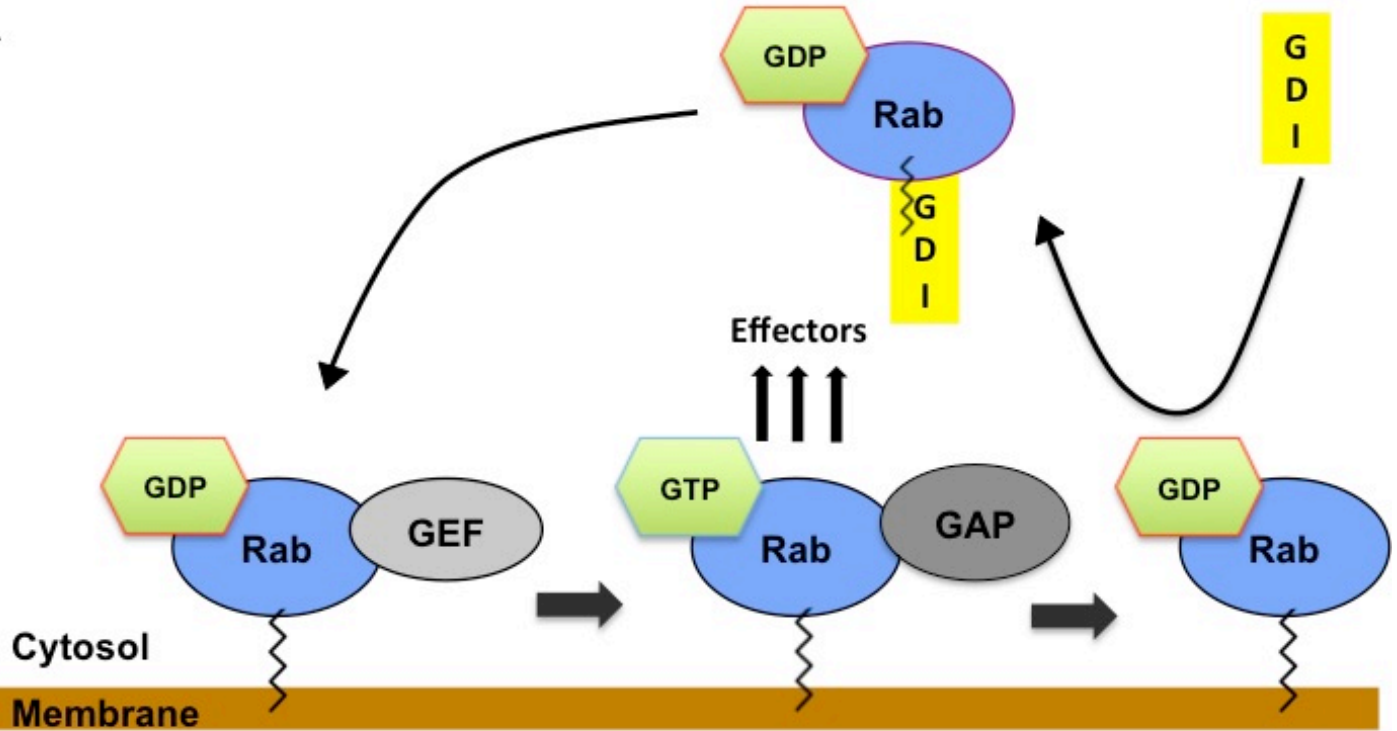

B

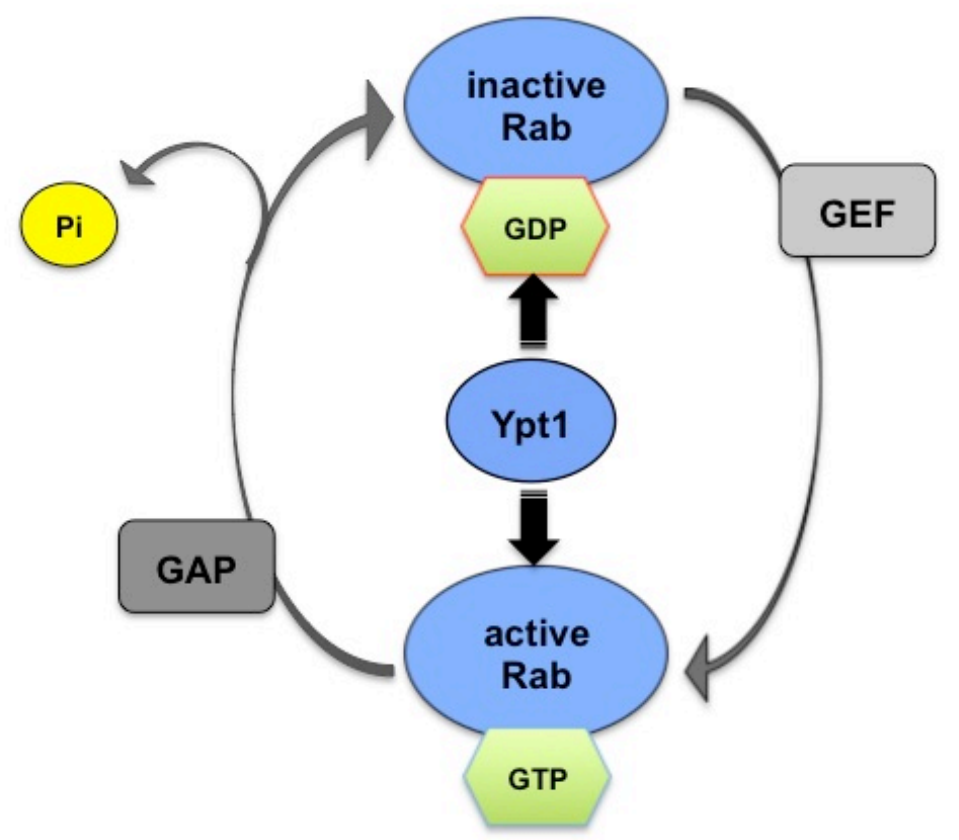

\section{Figure 2.2: The cycling of Rabs}

(A) Ypt/Rab proteins exist in soluble and membrane anchored populations. After dissociation of the GDIs (guanine-nucleotide dissociation inhibitors), the Rab can attach to the membrane by a geranylgeranyl group. The membrane-bound $\mathrm{Rab}$ is activated by a GEF (guanine-nucleotide exchange factor) and can interact with specific effectors. The Ypt/Rab is inactivated by GAPs (GTPase activating proteins), which hydrolyze the GTP to GDP.

(B) In the autophagic process, Ypt1 can be activated by its GEF TRAPPIII and switched off by its GAP. Thereby, Ypt1 is cycling from an activated GTP-bound form to an inactivated GDP-bound form. 
Guanine-nucleotide dissociation inhibitors (GDIs) prevent membrane binding of the soluble GTPase population (Pylypenko et al., 2006) and control the access of the GTPase to its regulatory elements, the GEFs and GAPs. GDIs are replaced by GDP dissociation factors (GDFs) to allow activation of the GTPase by its GEF (Fig. 2.2A). The GEF-mediated exchange of nucleotides makes the Rab resistant to extraction by the GDI. GDIs and GEFs thereby support the accumulation of Ypt/Rab GTPases on specific membranes. The system enables the assembly of unique membrane domain compositions that are limited in time. Newly synthesized Rab proteins are delivered to their destination membrane by Rab escort proteins (REPs) (Stenmark, 2009; Wandinger-Ness and Zerial, 2014).

In yeast, 11 Rabs have been identified, while humans have more than 70 (Pylypenko et al., 2017). Sequence analysis of Ypt/Rabs revealed high levels of conservation across different species that might even have evolved from an ancestral set of Rabs (Pereira-Leal and Seabra, 2001). The Rab Ypt1 is an essential protein that seems to mediate more than only one step in vesicle transport. It has been shown to regulate ER-to-Golgi and intra-Golgi transport and to play a role in autophagy (Jedd et al., 1995; Lipatova et al., 2012; Segev, 1991; Segev and Bernstein, 1987; Segev et al., 1988). This raised the idea that Ypt/Rabs and their respective effectors are specific for their particular compartment and not for the transport step (Lipatova et al., 2015). For example, Ypt1 and Ypt31/Ypt32 have been reported to be specific for the ER-derived membranes and the trans-Golgi (Segev, 2001).

\subsection{Autophagy}

Autophagy is a degradative process that is conserved among all eukaryotic cells. Proteins that are involved in the autophagic process are termed Autophagyrelated (Atg) proteins and in the last decades about 41 Atg proteins were identified (Farré et al., 2016; Yao et al., 2015). By the use of yeast genetics, a number of apg (autophagy) and aut (autophagocytosis) mutants have been identified as well as cvt (cytoplasm-to-vacuole targeting) mutants, which are defective in the transport of aminopeptidase 1 (Ape1) to the vacuole. Later analysis showed that these genes largely overlap, and the nomenclature has been unified as ATG (Klionsky et al., 2003). 


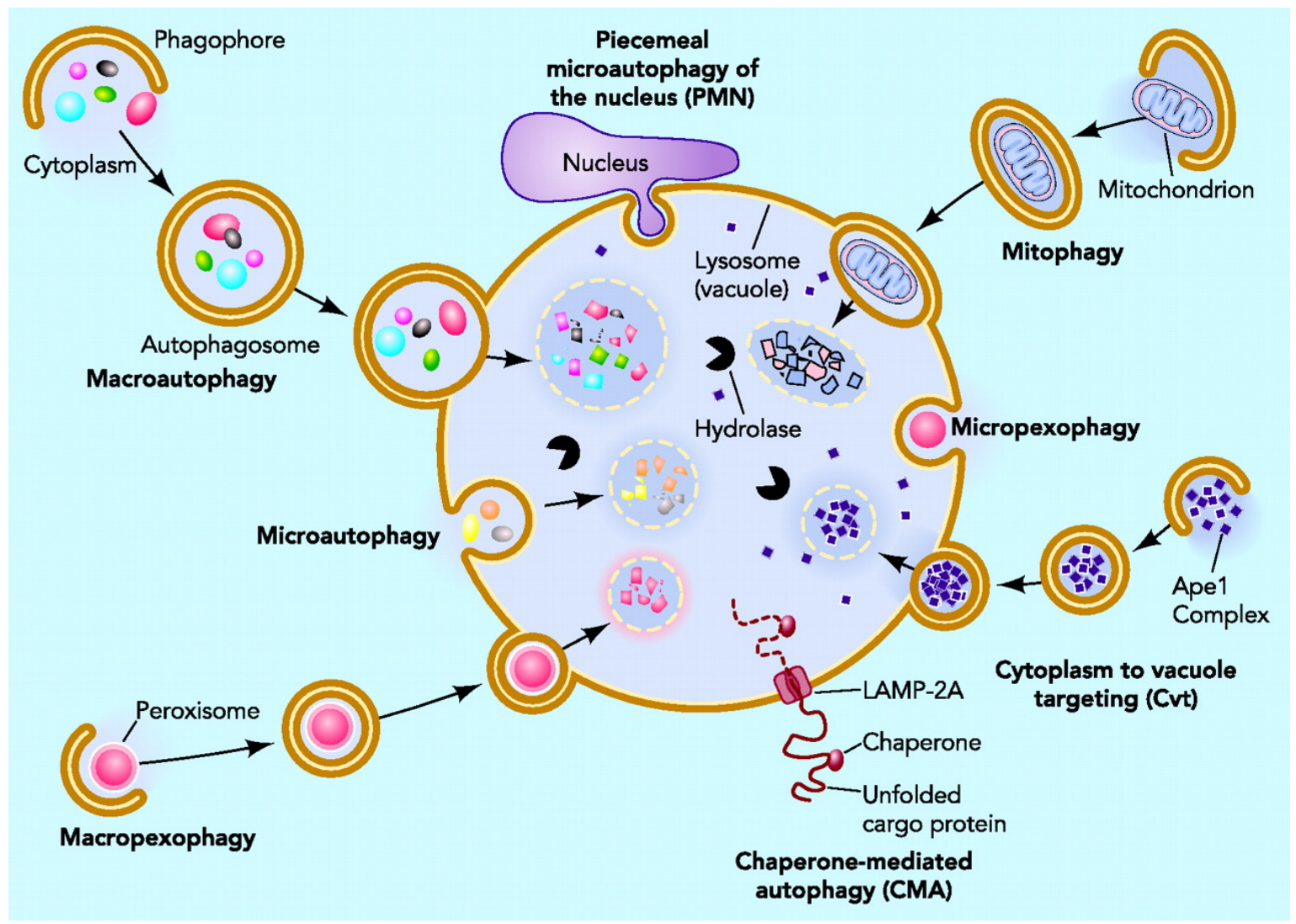

Figure 2.3: Types of autophagy (Yen and Klionsky, 2008)

During macroautophagy, proteins and organelles are selectively or unselectively enclosed by the forming autophagosome and transported to the vacuole in yeast or to the lysosome in mammals. There are different types of selective macroautophagy like macropexophagy, macromitophagy and the Cvt-pathway. During microautophagy, the cargo is directly engulfed by the vacuolar membrane. This form of autophagy has also various selective forms like piecemeal microautophagy of the nucleus (PMN). Chaperone-mediated autophagy is characterized by a special cargo recognition motive and only found in mammals up to now.

Autophagy is defined as a specific transport mechanism of proteins to the degradative organelles, which are the vacuole in yeast and plants and the lysosome in higher eukaryotes. Autophagy was initially studied as a response to stress conditions especially starvation, but it plays an important role in general cellular homeostasis (Choi et al., 2013). Autophagy is involved in adaption to stress conditions, aging, immunity, host defense and programmed cell death (Deretic et al., 2013; Filfan et al., 2017). The contribution in those processes leads also to several diseases that are associated with autophagy. Cancer, neurodegeneration, pathogen infection, myopathies and diabetes are known to be linked to an impaired degradation of superfluous or damaged proteins and thus 
they are linked to dysfunctions in the autophagic process (Jiang and Mizushima, 2014). There are different subtypes of autophagy: macroautophagy, microautophagy and chaperone-mediated autophagy (Fig. 2.3).

\subsubsection{Macroautophagy}

Macroautophagy (hereafter autophagy) is characterized by de novo formation of a double membrane-layered vesicle that transports its contents to the degrading compartment and can be selective and non-selective. First, a cup-shaped membrane, the phagophore, is formed, which expands and closes around its cargo to form the final double-membrane layered autophagosome (Yorimitsu and Klionsky, 2005). Proteins needed for the formation of the autophagosome assemble at the pre-autophagosomal structure (PAS). For the formation of autophagsomes, different membrane sources are under debate like the Golgi apparatus, the endoplasmic reticulum (ER), mitochondria and the plasma membrane (Lamb et al., 2013; Chan et al., 2013; Sanchez-Wandelmer et al., 2015). The ER was one of the first membrane sources under debate (Dunn, 1990) and later studies even identified a direct connection between the ER and the forming autophagosome in mammals. These studies also postulated that there should be other membrane sources besides the ER (Hayashi-Nishino et al., 2009; Ylä-Anttila et al., 2009). Thus, the origin of the autophagosomal membrane is one of many questions in the field that is still unanswered.

During autophagy, the outer membrane of the autophagosome fuses with the vacuole and releases the single membrane-layered vesicle, the autophagic body, into the vacuole. Here, the membrane is lysed and its contents are degraded or processed by vacuolar hydrolases. Under starvation, this process is highly upregulated, but also constitutively proceeds under growing conditions. Formation of the autophagosome takes 4-5 $\mathrm{min}$ in yeast and the estimated time for one autophagic cycle from initiation of the autophagosome to its degradation inside the vacuole is 7-9 $\min$ (Geng et al., 2008). The diameter of the autophagosome is about 0.3 to $0.9 \mu \mathrm{m}$ in yeast (Baba et al., 1997). Besides the unselective degradation of bulk cytoplasm, there are also selective variants of autophagy (Fig. 2.3), which are characterized by selective receptor proteins (Zaffagnini and Martens, 2016). Selective forms of autophagy (e.g. Cvt-pathway) exclude 
unspecific components and cytoplasm to form the transport vesicle closely around the cargo (Reggiori and Klionsky, 2013). Another form of autophagy is chaperonemediate autophagy (CMA), which mediates the degradation of a selective subset of soluble proteins. CMA is characterized by a special recognition motif KFERQ (lysine, phenylalanine, glutamic acid, arginine, glutamine) (Dice, 1990), which is recognized by a cytosolic chaperone that targets the protein to the surface of lysosomes where it binds to LAMP-2A (Lysosome-associated membrane protein 2) (Chiang et al., 1989). CMA has been detected in most mammalian cell types and seems to be absent in yeast (Kaushik and Cuervo, 2009).

\subsubsection{Microautophagy}

During microautophagy, the cargo is directly engulfed by the vacuolar membrane. This process can be selective or unselective and is like macroautophagy induced under nutrient limitation. Unselective microautphagy engulfs substrates by forming tubular invaginations. Selective forms of microautophagy are piecemeal of the nucleus (PMN), micropexophagy and micromitophagy (Li et al., 2012; Krick et al., 2008).

\subsection{Selective forms of autophagy}

\subsubsection{The Cvt-pathway}

The cytoplasm-to-vacuole targeting pathway (Cvt-pathway) transports resident hydrolases to the vacuole by using the autophagic machinery (Fig. 2.4). The precursor aminopeptidase I (pApe1 or prApe1 in figure 2.4), the best-studied cargo of the Cvt-pathway, is synthesized in the cytosol where it assembles into a dodecamer. pApe1 is synthesized with an N-terminal propeptide, which mediates the assembly of the dodecamers into large oligomers, the Ape1 complex. In contrast to unselective macroautophagy, the cargo is exclusively enclosed by the Cvt-vesicle (Sawa-Makarska et al., 2014). 


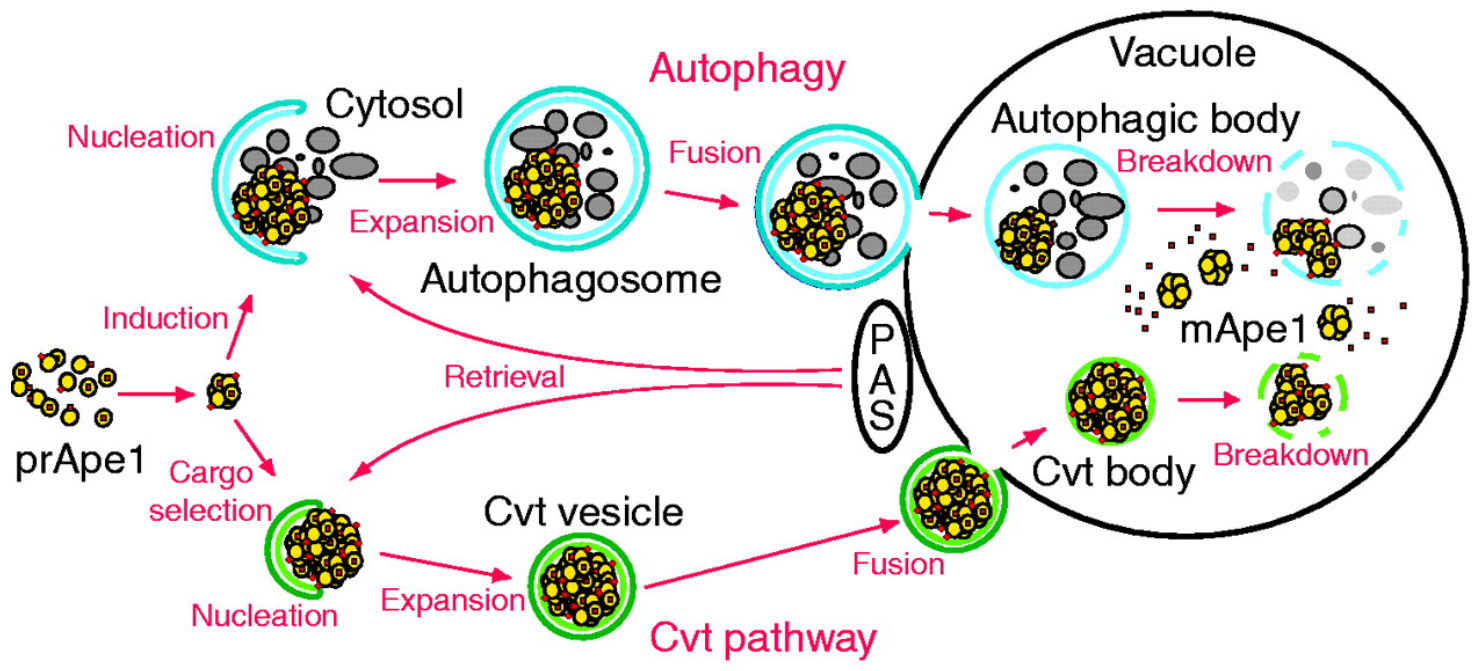

Figure 2.4: The Cvt-pathway and unselective autophagy in yeast (Klionsky, 2005)

Under nutrient-rich conditions, the precursor form of Ape1 (prApe1) is enclosed by the Cvt-vesicle and transported to the vacuole where it fulfills its function in the degradation of proteins. These vesicles are much smaller compared to autophagsomes and their targets are tightly enclosed by the isolation membrane. The Cvt-pathway uses the autophagic machinery. Upon starvation, prApe1 is transported by the autophagosome, which engulfs besides prApe1 other cytoplasmic components for degradation.

The Ape1 complex binds its receptor Atg19 that targets the complex to the PAS where pApe1, Ams1 (a-mannosidase) and Ape4 (aspartyl aminopeptidase) are enclosed by the Cvt-vesicle (Lynch-Day and Klionsky, 2010). It has been shown that Atg19 interacts with the cargo first, then with Atg11 and finally with Atg8, which links the complex to the phagophore (Scott et al., 2001; Yorimitsu and Klionsky, 2005; Shintani et al., 2002). Furthermore an independent and dual interaction of Atg19 with Atg11 and Atg8 is proposed (Chang et al., 2007). The Cvt-vesicle is transported to the vacuole where the outer membrane fuses with the vacuolar membrane to release the Cvt-body. In the vacuole, pApe1 is processed (by cleaving off its propeptide) into the mature form, mApe1 (Klionsky et al., 1992). pApe1 has a half-life of $45 \mathrm{~min}$ and is an ideal marker for monitoring autophagy (Klionsky et al., 1992). The size of Cvt-vesicles is about 140-160 nm in diameter and thus they are much smaller than an autophagosome (Baba et al., 1997). 


\subsubsection{Mitophagy}

Mitochondria are organelles that can be found in all eukaryotic organisms. Their main function is the generation of ATP (adenosine triphosphate) that provides energy for the cell. Furthermore, mitochondria are needed for biosynthesis of protein cofactors (Stehling and Lill, 2013), cellular differentiation and cell death (Li et al., 2017). For the production of ATP, an electrochemical proton gradient across the mitochondrial membrane is generated. The protons pass the ATP synthase complex, leading to ATP synthesis by oxidative phosphorylation. During this process, reactive oxygen species emerge as a by-product. Reactive oxygen species harm the mitochondria, thereby leading to apoptosis of the cell, neurodegeneration or cancer (Wu et al., 2016). Turnover of damaged and superfluous mitochondria is an important process to maintain cell viability and is termed mitophagy. Defects in mitophagy are linked to various diseases such as Parkinson's disease, heart failure or aging-related diseases (Yamaguchi et al., 2016). The basic mechanisms of mitophagy in S. cerevisiae are poorly described. A screen, analyzing yeast deletion strains, discovered Atg32 as a protein that affects only mitophagy but not unselective macroautophagy or other selective types of autophagy (Kanki et al., 2009; Okamoto et al., 2009).

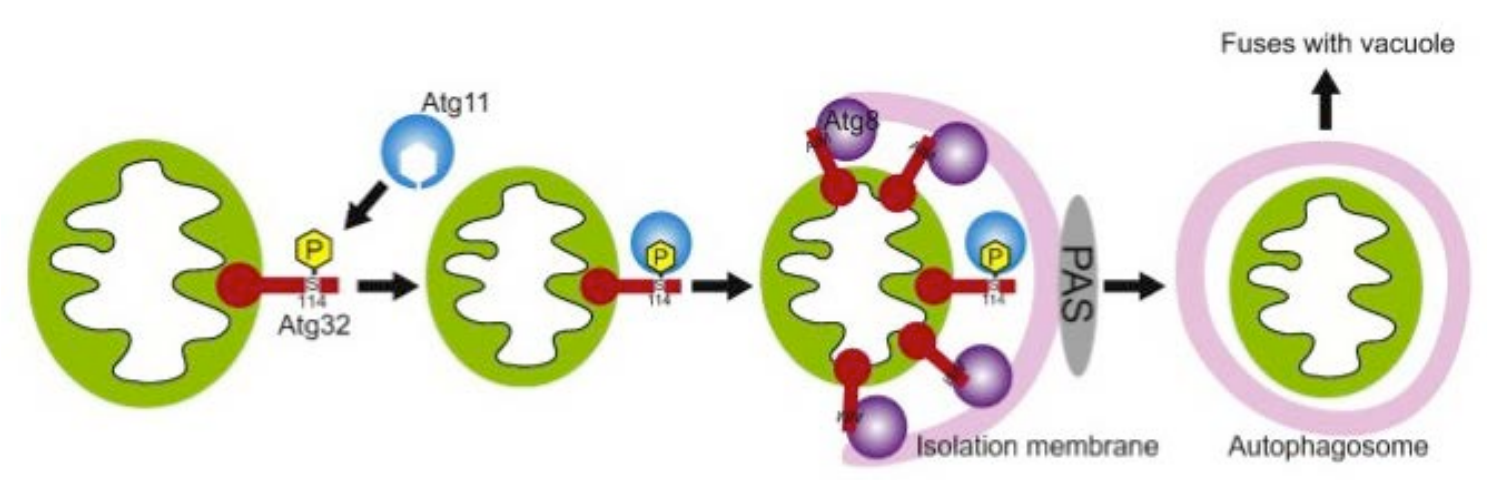

Figure 2.5: Mitophagy in yeast (modified after Kanki et al., 2015)

Atg11 targets the mitochondria to the PAS. Atg8 interacts with Atg32 in an AIM (Atg8 interacting motif)-dependent manner and anchors the mitochondria to the isolation membrane, which then encloses the mitochondria. Finally, the autophagosome fuses with the vacuole and the enclosed cargo is degraded.

Atg32 functions as selective receptor in mitophagy and is upregulated under respiratory growth conditions. It is located at the outer membrane of the mitochondria and exposes its $\mathrm{N}$-terminal domain to the cytosol and its $\mathrm{C}$-terminal 
domain to the intermembrane space (Fig. 2.5) (Okamoto et al., 2009). The cytosolic domain contains an Atg11 interaction domain and an Atg8 interacting motif (AIM) for binding to Atg8 (Farré et al., 2013). AlMs were detected in various selective autophagy receptors and are characterized by a specific sequence motif (Noda et al., 2010). Via these AIMs, Atg8 links the cargo receptors and their cargos to the forming autophagosome. Phosphorylation close to AIMs regulates the interaction of the cargo receptors to Atg8 and to the scaffold protein Atg11 (Farré et al., 2013). Upon mitophagy induction, Atg32 is phosphorylated. Especially the phosphorylation of serine 114 has a proposed role in the interaction of Atg32 and Atg11, but does not affect Atg8 interaction (Fig. 2.5) (Aoki et al., 2011).

\subsection{The PAS and the core machinery of autophagy}

The PAS (pre-autophagosomal structure or phagophore assembly site) is the proposed side where the phagophore assembles (Suzuki et al., 2001). The PAS is a constitutively formed structure and under growing conditions characterized by Atg11. By the use of fluorescence microscopy, the PAS has been defined as an accumulation of Atg proteins that localize close to the vacuole. Atg proteins are recruited to the PAS in a hierarchical manner (Suzuki et al., 2007) and they localize at the PAS, at least transiently. 18 Atg proteins have been described to belong to the core machinery. These proteins are needed for all kinds of autophagy (Suzuki and Ohsumi, 2010; Suzuki et al., 2017). Autophagy can be divided into functional subgroups: Atg1 complex, phosphatidylinositol 3-kinase complex, Atg9 vesicle associated complex and two ubiquitin-like conjugation systems. The subgroups will be described in the following chapters.

\subsubsection{The Atg1 complex}

The Atg1 complex consists of Atg1, Atg13, Atg17, Atg31 and Atg29 (Fig. 2.6A). Atg1 and Atg13 are recruited to the PAS upon starvation, while the Atg17-Atg31Atg29 complex is constitutively located at the PAS (Davies et al., 2015; Stjepanovic et al., 2014). Formation of the Atg1 complex is one of the earliest events after autophagy induction. Induction of autophagy is regulated by the target of rapamycin complex I (TORCl), which regulates Atg1. TORCl is highly conserved and consist of Tor1 and Tor2, Kog1, Lst8 and Tcp89 in yeast (Loewith 
and Hall, 2011). The TORCl negatively regulates autophagy in response to nitrogen levels in the cell. Under nutrient-rich conditions, the complex is active and hyperphosphorylates Atg13 and Atg1 (Kamada et al., 2010). Autophagy can be induced by starvation or treatment with rapamycin, a lipophilic antifungal agent, isolated from Streptomyces hygroscopicus. Rapamycin is able to induce autophagy even under nutrient-rich conditions by inhibiting TORCI (Vézina et al., 1975; Barbet et al., 1996). Protein kinase A (PKA) is a further regulator of autophagy that is able to sense the glucose level in the cell. PKA phosphorylates Atg13 similar to TORC1 but at different sites (Stephan et al., 2009).

A

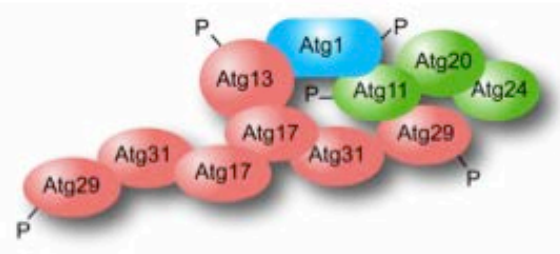

$\mathrm{B}$

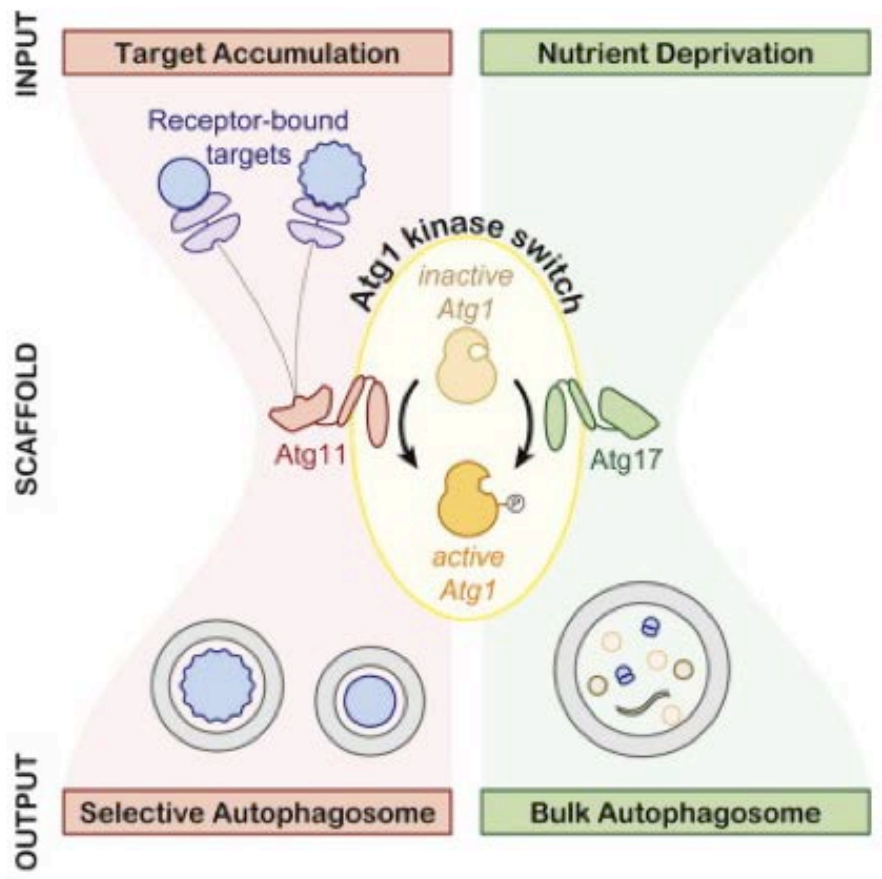

Figure 2.6: The Atg1 kinase complex

(A) Atg13 is phosphorylated under nutrient-rich conditions and gets dephosphorylated upon autophagy induction. Atg13 then interacts with Atg17, a scaffold protein that dimerizes and forms a ternary complex with Atg29 and Atg31. The Atg17-Atg31-Atg29 complex replaces the scaffold function of Atg11 under starvation conditions. The two sorting nexins Atg20 and Atg24 interact via Atg11 with the complex (Reggiori and Klionsky, 2013).

(B) Under nutrient-rich conditions, Atg1 is activated by a target protein bound to its receptor and the scaffold protein Atg11. This leads to induction of selective autophagy (Kamber et al., 2015). 
Atg1 is a serine/threonine kinase (Matsuura et al., 1997) that is able to autophosphorylate and to phosphorylate its targets. For optimal function of Atg1, Atg13 is required (Kamada et al. 2000). Atg13 is considered as a sensor for autophagy. Under nutrient-rich conditions Atg13 is hyperphosphorylated, while it gets dephosphorylated under starvation conditions (Scott et al. 2000). This dephosphorylation of Atg13 may lead to a high affinity interaction of Atg1 and Atg13 and thus to activation of the Atg1 complex (Kamada et al., 2000). Besides the model of interaction upon nitrogen starvation, other data show that Atg1 and Atg13 are always in a complex (Kraft et al., 2012). This finding is in line with the fact that the mammalian Atg1 homolog ULK1 is constantly assembled to Atg13. Atg13 interacts furthermore with Atg17. Atg17 is a S-like shaped scaffold protein that can dimerize and form a ternary complex with Atg29 and Atg31, whereby Atg31 bridges the other proteins (Fig. 2.6A) (Cao et al., 2009, Kabeya et al., 2009).

Atg17 interacts most likely directly with Atg13 but also interacts with Atg29 and Atg11 (Yorimitsu et al., 2005). The crystal structure of Atg17 revealed the assembly of the protein into a dimer that exhibits a coiled-coil domain and a curved scaffold structure. Atg1 and Atg17 might provide an initial structure for nucleation of the phagophore (Ragusa et al., 2012). The Atg17-Atg31-Atg29 complex is important for the recruitment of further Atg proteins and membrane sources like Atg9 vesicles (Mao et al, 2013; Stanley et al., 2014). In the absence of Atg17 and Atg11, assembly of other Atg proteins is not detectable (Suzuki et al., 2007).

Under growing conditions, Atg11 is the first component that localizes to the PAS and recruits the Atg1 complex. Upon autophagy induction, Atg11 is replaced by the Atg17-Atg31-Atg29 subcomplex that substitutes the scaffold function of Atg11 under starvation conditions (Cheong et al., 2007; Mao et al., 2013). Atg20 and Atg24 are two sorting nexins that interact via Atg11 with the complex and both proteins also bind phosphatidylinositol 3-phosphate.

The Atg1 kinase activity is essential for the Cvt-pathway, but repressed under nutrient-rich conditions by the nutrient-sensing pathway. Thus, the induction of the Cvt-pathway is mechanistically different from the induction of unselective and 
starvation-induced autophagy. The less catalytically active Atg1 might be sufficient for the formation of Cvt-vesicles. Another possibility is that cargo-bound receptors like pApe1-Atg19 use the interaction with the scaffold Atg11 to activate Atg1. Thus, selective targets of autophagy may activate Atg1 and bypass Atg1 repression (Fig. 2.6B) (Kamber et al., 2015).

\subsubsection{Phosphatidylinositol 3-kinase complex}

There are two phosphatidylinositol (PtdIns) 3-kinase complexes known that direct the synthesis of phosphatidylinositol 3-phosphate (PI3P) in yeast. Ptdlns 3-kinase complex I is essential for autophagy and PtdIns 3-kinase complex II is involved in endosomal trafficking, endocytosis and the vacuolar protein-sorting pathway (Kihara et al., 2001; Obara and Ohsumi, 2011). In autophagy, PI3P serves as a platform to recruit proteins for phagophore formation. Both complexes consist of Vps15, Vps34 and Vps30. Complex I additionally includes Atg38 (Araki et al., 2013) and Atg14. Atg14 targets the complex to the PAS where it is required for the localization of Atg proteins like Atg8, Atg2, Atg5 and Atg18 (Kihara et al., 2001; Shintani et al., 2001; Suzuki et al., 2001; Krick et al., 2006; Obara et al., 2006). In complex II, Vps38 replaces Atg14. Under logarithmic growth conditions, the level of PI3P at the PAS is very low. The recruitment of Atg14 to the PAS is currently under debate. It is reported that Atg14 is recruited to the PAS by the HORMA domain of Atg13 (Jao et al., 2013) but the HORMA domain of Atg13 is also reported to recruit Atg9 vesicles to the PAS (Suzuki et al., 2015). Nevertheless, Atg14 directs the Ptdlns 3-kinase complex I to the PAS (Obara et al., 2006).

\subsubsection{Atg9 containing vesicles}

One unanswered question in autophagy involves the origin of the membrane that builds the autophagosome. Several cellular structures and compartments like mitochondria or the ER are under debate to contribute to the membrane that is needed to form the autophagosome. A protein that is involved in the recruitment of membranes is the multispanning membrane protein Atg9 (Noda et al., 2000; Reggiori et al., 2004). Atg9 belongs to the core machinery of autophagy and is required for selective and unselective forms of autophagy. It is upregulated upon starvation and locates on highly mobile vesicles that seem to originate at the 
Golgi apparatus. Atg9 cycles between the PAS and peripheral structures during autophagy (Yamamoto et al., 2012; Mari et al., 2010). Mari et al. (2010) postulated that Atg9 locates in a novel compartment consisting of cytosolic clusters close to mitochondria, providing an Atg9 reservoir. Thus, the origin of Atg9 vesicles is still under debate but both authors agree that Atg9 translocates from the peripheral sites to the PAS. Upon induction of autophagy, Atg9 locates to the PAS where it contributes to nucleation of the phagophore and acts as part of the membrane precursor (Stanley et al., 2014) (Fig. 2.7). The cycling of Atg9 vesicles requires various proteins that regulate the anterograde and retrograde transport. Here, the Atg13-Atg1 signaling complex is needed, the PtdIns3-kinase complex, the Atg2-Atg18 complex, Atg23 and Atg27 (Reggiori et al., 2004; Backues et al., 2015; Legakis et al., 2007).

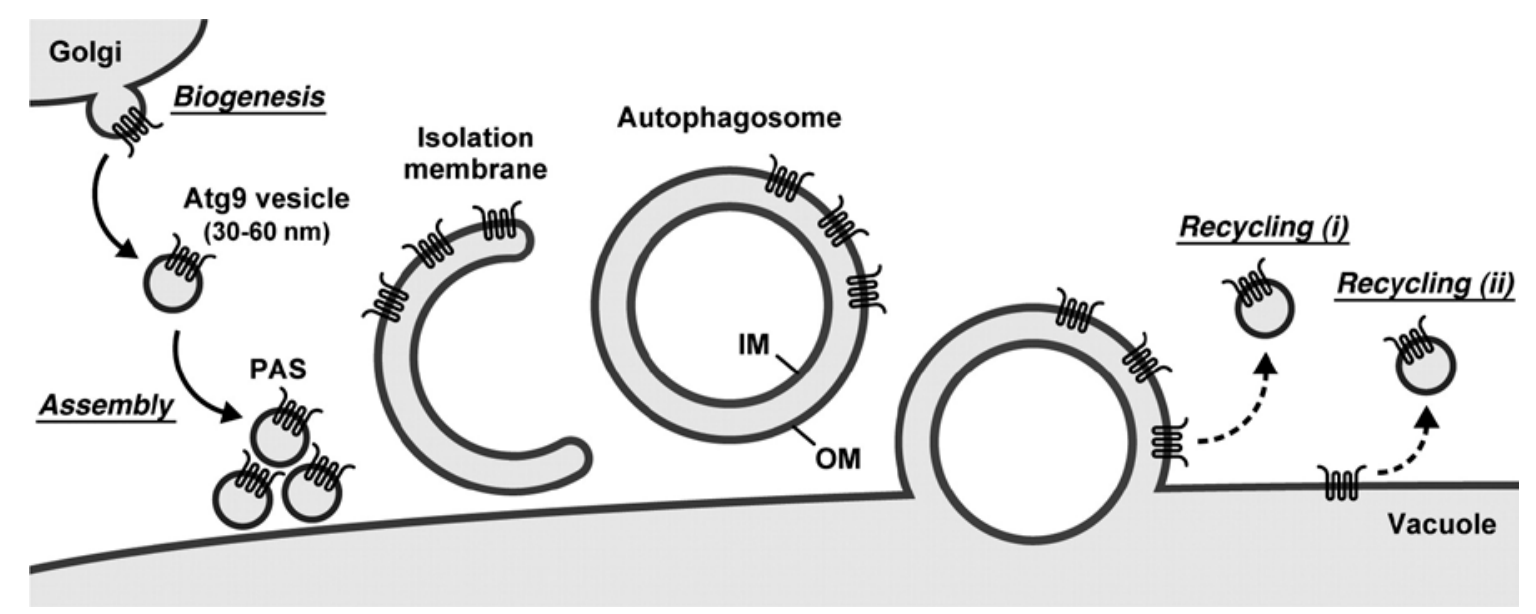

Figure 2.7: Cycling of Atg9 containing vesicles (Yamamoto et al. 2012)

The Atg9 protein is located on highly mobile vesicles that cycle from their origin to the PAS where they contribute to phagophore formation. The vesicles are removed and then recycled before the autophagosome fuses with the vacuole.

Studies using high-resolution microscopy revealed that three Atg9 vesicles assemble close to the vacuole and form the PAS (Yamamoto et al., 2012). Atg9 is not found on the completed autophagosome, indicating that it is removed prior to fusion with the vacuole (Noda et al., 2000). Calculation of the diameter of the Atg9 vesicles and the observation that the autophagic machinery is not supplied with further Atg9 vesicles in later steps of autophagy, led to the conclusion that they would only supply about $2 \%$ of the autophagosomal membrane (Yamamoto et al., 2012). Thus, additional membrane sources are needed. 
Atg9 furthermore co-localizes with the TRAPP (transport protein particle) III complex and directly interacts with its component Trs85. Ypt1 is not directly bound to Atg9, but located on Atg9 vesicles. Thus, Ypt1 is presumably transported to the PAS by Atg9 vesicles. TRAPPIII, the GEF of Ypt1, might be important to facilitate binding of Ypt1 to the membrane of Atg9 vesicles. Atg11 interacts with Ypt1 and Atg9 and might thereby target Atg9 vesicles to the PAS (Kakuta et al., 2012; Lipatova et al., 2012; He et al., 2006). Ypt1 furthermore interacts with Atg1, which uses Atg9 as substrate for phosphorylation. An Atg9 mutant protein that cannot be phosphorylated has been shown to cycle properly in cells but failed to recruit Atg18 and Atg8 (Papinski et al., 2014). Under nutrientrich conditions, Trs85 is important to recruit Atg14 and Atg8 to the PAS. This was not observed after rapamycin treatment (Kakuta et al., 2012).

\subsubsection{COPII mediated vesicle formation}

The role of Ypt1 has been intensively studied in targeting COPII (coat protein complex II) vesicles from the ER to the cis-Golgi (Barrowman et al., 2010; Davies and Ferro-Novick, 2015; Jedd et al., 1995). The formation of COPII vesicles is initiated by Sar1 at specialized domains of the ER, so-called ER-exit sites. Sar1 is a GTPase that becomes membrane anchored upon GTP-binding. It induces curvature of the membrane and recruits the inner COPII coat consisting of Sec23 and Sec24. These proteins then mediate recruitment of the outer shell consisting of Sec13 and Sec31 (Matsuoka et al., 1998). This leads to coat polymerization and budding of the COPII vesicle. After completing the full coat, Sar1 is inactivated by its GAP Sec23 and released from the vesicle. Due to release of Sar1, TRAPPI can bind to Sec23 and recruit and activate Ypt1 on the vesicle (Cai et al., 2007). Ypt1-GTP then recruits its effector Uso1, which links the vesicle to the cis-Golgi (Cao et al., 2000). At the Golgi, TRAPPI is displaced by Hrr25, which also phosphorylates Sec23 (Lord et al., 2011). These interactions thereby perform the directed transport of the COPII vesicles to the Golgi. After the vesicle has arrived at its destination, the outer coat is removed and recycled. Removing the outer coat is a prerequisite for membrane fusion that requires the formation of a SNARE (soluble N-ethylmaleimide-sensitive-factor attachment receptor) complex (Bhandari et al., 2013). 


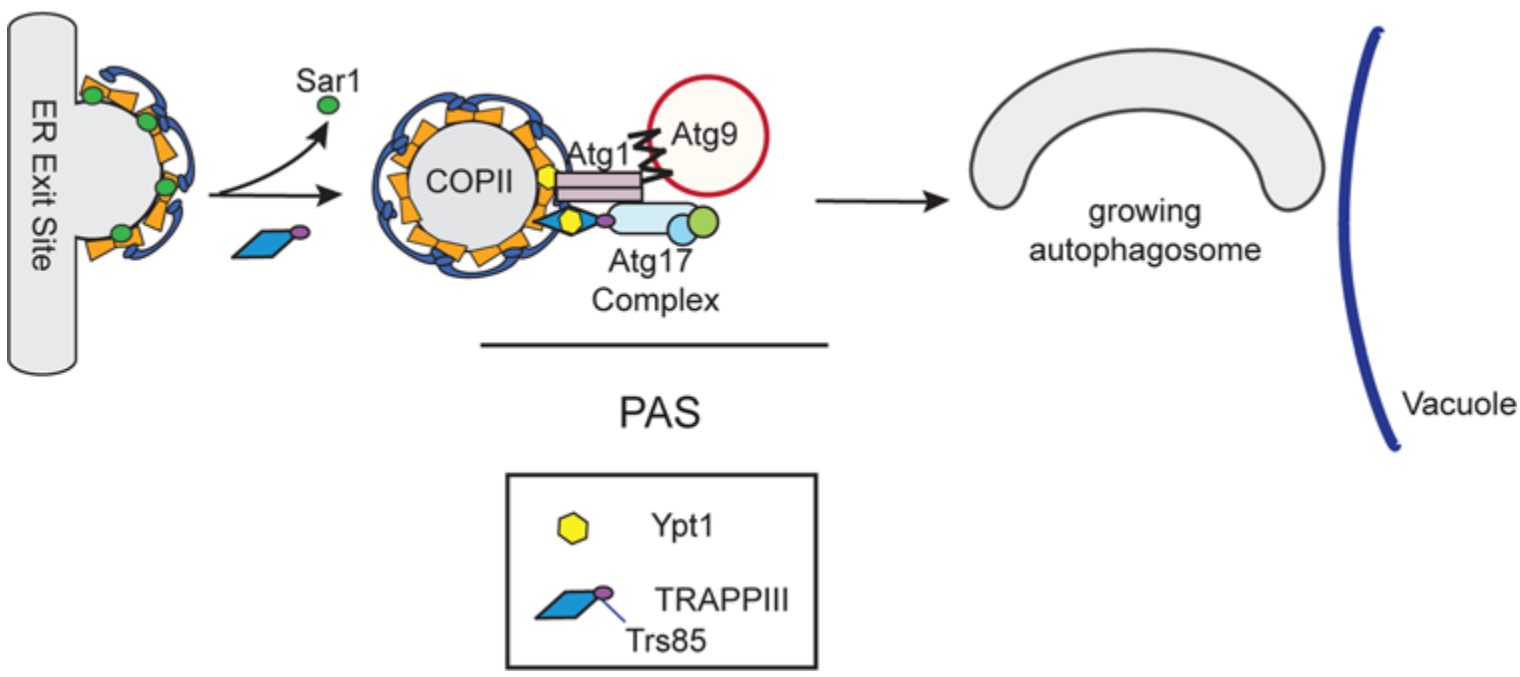

Figure 2.8: Role of COPII vesicles in autophagosome biogenesis (Davies and Ferro-Novick, 2015)

Sar1 initiates the formation of COPII vesicles at so-called ER-exit sites. The PAS is supposed to form close to ER-exit sites and the vacuole. Atg17 recruits the TRAPPIII complex to the PAS where it binds to the COPII subunit Sec23. Trs85, subunit of the TRAPPIII complex recruits and activates Ypt1, which plays a role in recruitment of Atg1. Atg1 may tether Atg9 and COPII vesicles and this may lead to initiation of phagophore formation.

COPII vesicles also appear to play a role in autophagy as they accumulate at the PAS when autophagy is blocked (Tan et al., 2013). Because Atg9 vesicles are not sufficient to generate a membrane needed for phagophore formation, other membrane sources are required. Subunits of the COPII vesicles have been demonstrated to interact with a variety of Atg proteins and the function of the COPII vesicles is supposed to be downstream of the Atg1 autophagy initiation complex (Graef et al., 2013). Many proteins that regulate COPII vesicle traffic from ER to Golgi are also involved in the regulation of autophagy like Sar1 and SNAREs but not the tether Uso1. Thus, COPII vesicles might be transported in autophagy by a similar mechanism but targeted to a different membrane by a different tether (Tan et al., 2013). It has been hypothesized that Atg1 functions as a tether and links the COPII vesicles to Atg9 vesicles (Davis and Ferro-Novick, 2015). Recently, an in vitro study demonstrated that Atg1 binds to curved membranes and is involved in tethering Atg9 vesicles (Rao et al., 2016). COPII vesicles are reported to contribute to autophagy only under starvation conditions (Wang et al., 2017). 


\subsubsection{Two ubiquitin-like protein conjugation complexes}

Atg8 and Atg12 are ubiquitin-like proteins, which are processed by the two ubiquitin-like protein conjugation complexes of autophagy (Gen and Klionsky, 2008). The Atg12-conjugate thereby functions in the formation of lipidated Atg8, which is important for elongation of the forming phagophore membrane (Fig. 2.9).

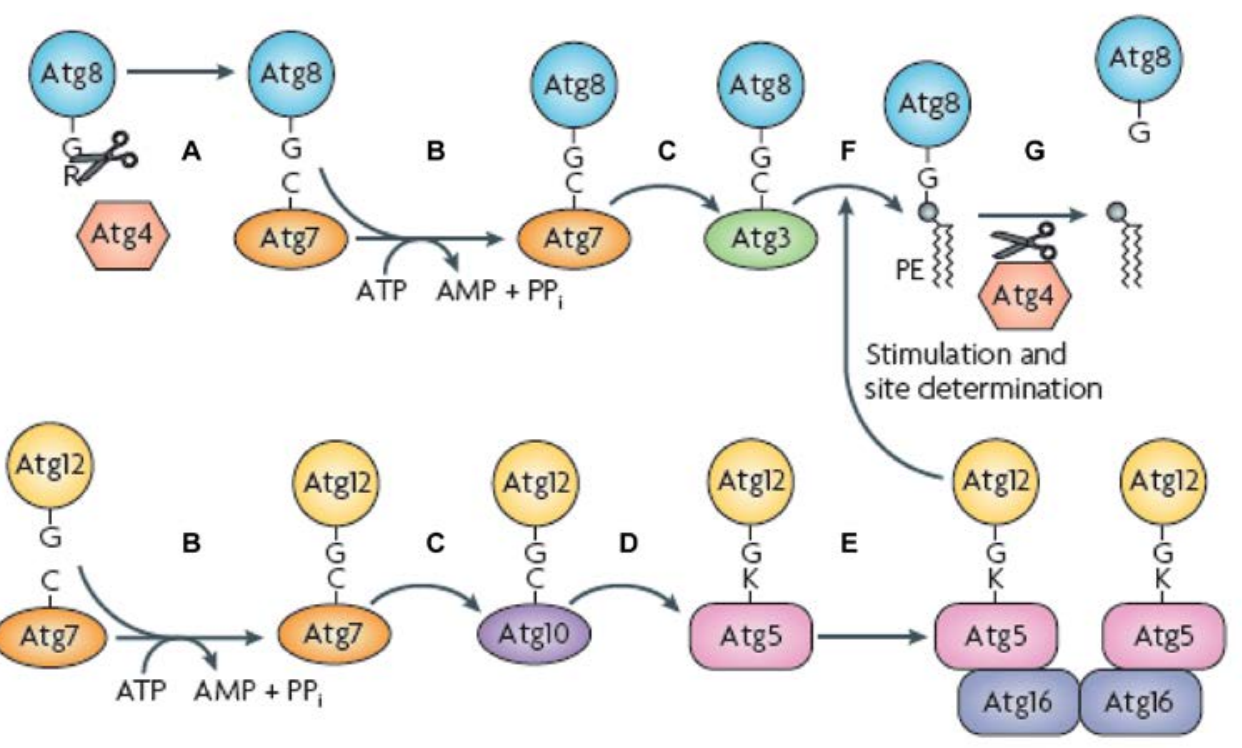

Figure 2.9: The ubiquitin-like conjugation of Atg8 (modified from Nakatogawa et al., 2009)

The conjugation of Atg8 to PE requires two ubiquitin-like conjugation processes. First, the C-terminal arginine of Atg8 is cleaved off by Atg4 (A) to allow activation by Atg7 (E1-like enzyme), which also activates Atg12 (B). Atg8 is transferred to Atg3 (E2-like enzyme) and Atg12 forms a complex with Atg10 (E2-like enzyme) (C) to become covalently linked to Atg5 (D). The complex of Atg12-Atg5/Atg16 has an E3-like function and stimulates the transfer of Atg8 to PE, thereby linking the two conjugation systems (F). To release Atg8 form the outer membrane of the autophagosome, Atg4 fulfills its second function and deconjugates Atg8 from PE (G). Then Atg8 can be reused (modified from Nakatogawa et al., 2009).

Atg8 is synthesized with a C-terminal arginine (R), which is removed by the cysteine protease Atg4 to allow Atg8 to interact via the terminal glycine (G) with the conjugation system (Kirisako et al., 2000). Atg7 (E1-like enzyme) activates the processed Atg8 in an ATP-consuming reaction and transfers Atg8 to Atg3, an E2like enzyme. Atg3 forms a covalent bond between the glycine residue of Atg8 and PE. Thereby, Atg8 is linked to both sides of the phagophore (Nakatogawa et al., 2007; Noda et al., 2008; Kaufmann et al., 2014). Similar to the first conjugation process, also Atg12 is activated by Atg7 (E1-like enzyme) and transferred to 
Atg10 (E2-like enzyme). Atg10 leads to the formation of a covalent bond between the C-terminal glycine of Atg12 and a lysine of Atg5. Compared to Atg8, the ubiquitin-like protein Atg12 is not processed before its activation (Mizushima et al., 1998). The resulting Atg12-Atg5 conjugate forms a complex with Atg16. The Atg12 Atg5/Atg16 complex acts as an E3-like enzyme for the final conjugation of Atg8 to PE. Atg16 is not needed for the E3-like function of the complex, but for the localization of the complex to autophagic membranes (Hanada et al., 2007; Noda et al., 2013). Interestingly, Atg8-PE can also be generated in the absence of the Atg12 Atg5/Atg16 complex (Cao et al., 2008).

\subsubsection{The ubiquitin-like protein Atg8}

Atg8 possesses an N-terminal helical domain and a C-terminal ubiquitin-like domain. It has several homologs in mammals that are grouped into three subfamilies: LC3 (light-chain 3), GABARAP ( $\gamma$-aminobutyric acid receptor-associated protein) and GATE-16 (Golgi-associated ATPase enhancer). LC3 is the bestcharacterized member of this family in mammals. LC3 is posttranslational processed into LC3-I (located in the cytosol) and LC3-II, which is conjugated to PE and associates with the autophagosomal membrane (Slobodkin and Elazar, 2013). Upon starvation, the expression of Atg8 is highly upregulated (about 10 times) and localization of Atg8 to the PAS increases. The amount of Atg8 determines the size of the autophagosome but does not limit the number of autophagosomes that are formed (Xie at al., 2008).

Besides its role in autophagosome formation, Atg8 is important for recruitment of cargos during selective forms of autophagy. Cargo receptors link the cargo to the isolation membrane by binding to the cargo and to Atg8 simultaneously (Stolz et al., 2014; Rogov et al., 2014). To mediate this binding, the cargo receptors contain highly conserved AIMs (Atg8 interacting motifs) that bind to the ubiquitinlike domain of Atg8 (Alemu et al., 2012). The position 0 of the motif requires an aromatic amino acid like tryptophan, phenylalanine or tyrosine and position +3 a hydrophobic residue like isoleucine, leucine or valine (Fig. 2.10A). Both positions are essential in contrast to adjacent residues, which are allowed to have more variability (Birgisdottir et al., 2013). The positions -1 and -2 are mostly acidic residues with aspartic acid or threonine and serine, which can be phosphorylated 
to increase the binding affinity and acidity. Position +1 of the core motif preferentially contains an acidic or hydrophobic residue (Fig. 2.10A) (Alemu et al., 2012). The consensus sequence is therefore defined as $[\mathrm{W} / \mathrm{F} / \mathrm{Y}] \mathrm{xx}[\mathrm{L} / / \mathrm{V}]$, where $x$ represents undefined amino acids. In mammals, these motifs are called LIRs (LC3-interacting regions) and interact with the Atg8 homologs LC3 and GABARAP (Fig. 2.10B). Potential AIM-like sequence motifs can be found in many proteins but not all of them are functional. Thus, other characteristics have been determined to identify the false positive AIMs. For example, the AIM is proposed to be a short linear motif in a disordered region (Popelka and Klinosky, 2015).

AIMs or LIRs bind to the two hydrophobic pockets in the ubiquitin-like domain of yeast Atg8 or its mammalian homologs (Fig. 2.10C). These hydrophobic pockets in Atg8, termed the W-site and the L-site, mediate the binding of Atg8 and its AIMcontaining interaction partner. Both pockets are composed of defined side chains and are responsible for the interaction with tryptophan/phenyalanine/tyrosine (Wsite) and leucine/isoleucine/valine (L-site) in the AIM (Noda et al., 2008; Alemu et al., 2012).

Various proteins were identified to interact with Atg8 via AlMs. Atg1 possesses an AIM in its center that interacts with Atg8. The interaction is thought to facilitate Atg1 linkage to the isolation membrane and its incorporation into the autophagosome. Mutation of these residues prevents enclosure of Atg1 by the autophagosome and led to defects in autophagy, indicating a function of Atg1 besides autophagy induction - in later steps of autophagy (Nakatogawa et al., 2012b; Kraft et al., 2012). The mammalian homolog of Atg1, ULK1/2 binds one of the Atg8 homologues GABARAB (gamma-aminobutyric acid receptor-associated protein) and might have a similar function at the phagophore in mammals (Kraft et al., 2012). The AlM-dependent interaction of Atg3 and Atg8 might promote Atg8 transfer to PE from the Atg3-Atg8 thioester intermediate (Yamaguchi et al., 2010). Atg19 is an example for a selective receptor that functions during the Cvt-pathway where it links Atg8 to the Cvt-vesicles. Atg32 has a similar function during mitophagy and links Atg8 to mitochondria (Okamoto et al., 2009). Further known receptors for selective autophagy are Atg34 (transport of Ams1), Atg36 
(pexophagy receptor), Atg39 (degradation of the ER and the nucleus) and Atg40 (degradation of the ER) (Farré and Subramani, 2016).

Under nutrient-rich conditions, the conjugation of Atg8 to PE is mediated by Atg21, which interacts with Atg16 and with the F5K6-motif in the N-terminal domain of Atg8. Depending on PI3P, Atg21 organizes efficient lipidation of Atg8 (Juris et al., 2015). On the outer surface of the autophagosome the Atg12Atg5/Atg16 complexes form a meshwork architecture-like membrane scaffold that seems to be stabilized by an AIM-dependent interaction of Atg8 with Atg12 (Fig. 2.10D). Atg8-PE/Atg12-Atg5 complexes are crosslinked by Atg16. This outer scaffold is supposed to support cargo delivery to the vacuole (Fig. 2.10E) (Kaufmann and Wollert, 2014).

Atg8 is linked to both sides of the phagophore. On the outer side, Atg8 is cleaved off in an Atg4-dependet cleavage step before fusion of the completed autophagosome with the vacuole. Thus, Atg4 functions not only in the processing of Atg8, but also in the deconjugation of Atg8 from PE and it's recycling from membranes. Different mechanisms are under debate how the activity of Atg4 is regulated to direct the liberating step of Atg8 and to prevent its cleavage from PE at the inner side of the forming autophagosome. Atg8 conjugation to PE might initially occur at different membranes in the cell. Atg4 might cleave Atg8 from PE from all this membranes except from those that form the autophagosomal membrane. Thereby, Atg4 would maintain a reservoir of unlipidated Atg8 that is conjugated to the autophagosome by PE and required for formation of the autophagosome (Nakatogawa et al., 2012a). Atg1 is reported to inhibit premature cleavage of Atg8-PE at the PAS by phosphorylating Atg4, which is then no longer able to process Atg8. Upon closure of the autophagosome and dissociation of the Atg1 complex, Atg 4 is no longer phosphorylated by Atg1 and able to cleave Atg8 from PE (Sánchez-Wandelmer et al., 2017). 


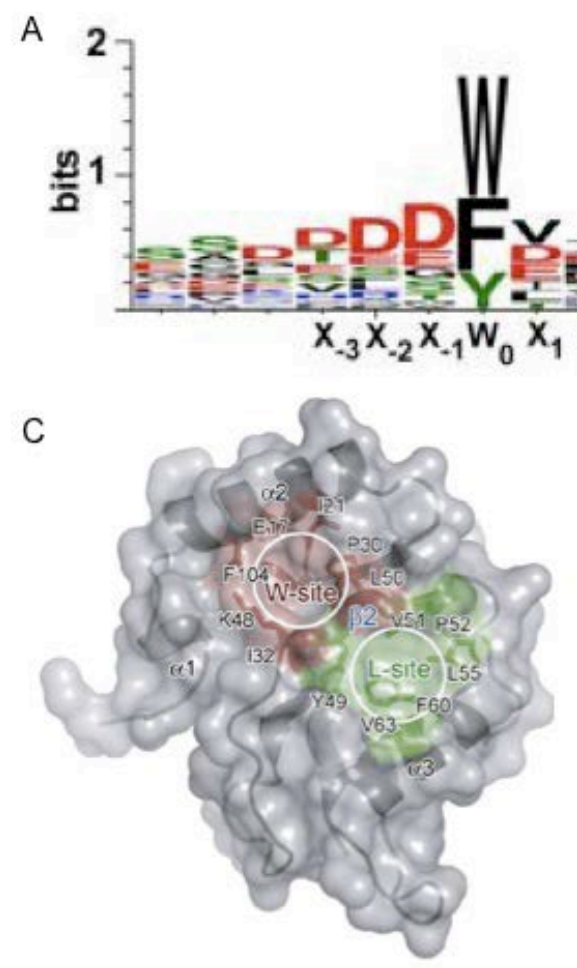

B

$\begin{array}{lll}\text { Atg19 } & \text { AlM sequence } & \text { Binding partner } \\ \text { Atg32 } & \text { SGSWELI } & \text { Atg8 } \\ \text { Atg3 } & \text { VGDWEDLQD } & \text { Atg8 } \\ \text { Atg8 } 8 \\ \text { P62 } & \text { TLTYDTLRF } & \text { LC3 } \\ \text { NBR1 } & \text { DDDWTHLSS } & \text { LC3 } \\ \text { SEDYIIILP } & \text { LC3 } \\ \text { CRT } & \text { EDDWDFLPP } & \text { GABARAP } \\ \text { CHC } & \text { TPDWIFLLR } & \text { GABARAP } \\ \text { Nix } & \text { NSSWVELPM } & \text { GABARAP } \\ \text { (PSSM) } & \text { DDDWVFVP } & \text { GABARAP } \\ \text { SEPA-1 } & \text { (TYGYQELDD) } & \text { LGG-1 }\end{array}$

D

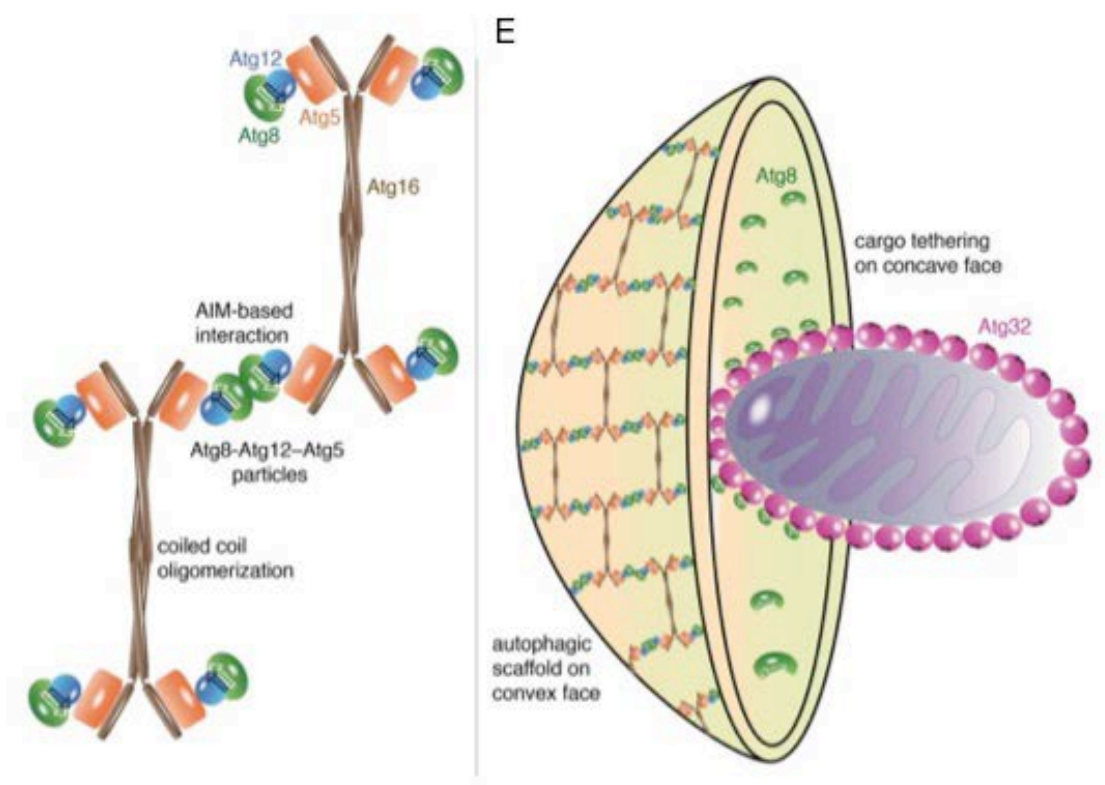

Figure 2.10: Atg8 interacts via AIMs and is initially located at both sides of the forming phagophore

(A) Many interaction partners of Atg8 interact with Atg8 in an AIM-dependent manner. The AIM is characterized by a sequence motif with an aromatic residue on position 0 that interacts with the W-site of Atg8 and a hydrophobic residue on position +3 that interacts with the L-site of Atg8. Further residues close to these positions were also statistically determined and their likeliness is indicated by the height of the letters (Alemu et al., 2012).

(B) Sequence alignment of identified AIMs that either bind Atg8 or its human homologs LC3 and GABARAP (Noda et al., 2010). 
(C) Structural analysis of Atg8 revealed two hydrophobic pockets (Noda et al., 2010). Via these pockets, Atg8 binds to its AIM-containing interaction partners.

(D) Atg8 is thought to interact in an AIM-dependent manner with Atg12, recruiting the Atg12 Atg5/Atg16 complex to the phagophore (Kaufmann et al., 2014).

(E) On the convex side of the phagophore, Atg8 contributes to scaffold formation and stability together with Atg12 Atg5/Atg16. On the concave side it functions as a cargo receptor by binding to Atg32 in an AIM-dependent manner (Kaufmann and Wollert, 2014).

Atg4 has furthermore two proposed AIMs (Atg8-interacting motif) that interact with Atg8. One AIM is supposed to bind Atg8 constitutively, the other one recognizes specifically Atg8-PE. Mutation of both AIMs leads to inability of Atg4 to cleave Atg8 from PE (Abreu et al., 2017). These findings are in line with the identification of a C-terminal LIR (LC3-interacting region) in mammalian Atg4B, which is also important for binding and cleavage of the mammalian Atg8 homologs LC3/GABARAP from PE (Skytte Rasmussen et al., 2017). The deconjugation process of Atg8 from PE seems to be important for several events in later stages of autophagy like disassembly of other Atg proteins from the PAS (Nair et al., 2012; Nakatogawa et al., 2012a).

After disassembly of Atg8 from PE on the outer side of the phagophore, the autophagic body can finally be released into the vacuole. The membrane is lysed and the contents can be degraded.

\subsection{Interplay of autophagy and GTPases}

Various membrane sources are currently under debate to contribute to the de novo formation of the autophagosome (Tooze, 2013; Mari et al., 2011). The specific and directed transport of membranes and vesicles that contribute to autophagy needs to be tightly controlled. Here, Ypt/Rab GTPases seem to play a key role (Szatmári and Sass, 2017).

Which GEFs activate Ypt1 for its different functions is currently highly under debate. So far, the TRAPPIII complex, defined by its specific subunit Trs85, has been considered to recruit Ypt1 to the phagophore during autophagy (Lynch-Day et al., 2010; Lipatova et al., 2012; Kakuta et al., 2012). Trs85 is assumed to direct the TRAPPIII complex to the phagophore, which also depends on Atg17. Ypt1 and Trs85 are further assumed to interact on Atg9-containing vesicles, thereby 
promoting PAS assembly (Lipatova and Segev, 2012). Two other TRAPP complexes activate Ypt1 on the other pathways: TRAPPI: ER-Golgi, TRAPPII: intra-Golgi (Barrowman et al., 2010). Recently, a new TRAPPIV complex has been identified that contains Trs33 (so far considered as a TRAPPI/II subunit) as specific component. TRAPPIV is also proposed to recruit Ypt1 to the PAS (Lipatova et al., 2016). TRAPPIII might be the main GEF of Ypt1 or the two TRAPP complexes might be required for different autophagic subtypes (Lipatova et al., 2016). This dual recruitment of Ypt1 could also explain the weak phenotype observed for the TRS85 deletion under starvation (Lynch-Day et al., 2010). Another recent study proposes that there are only two TRAPP complexes, TRAPPII and TRAPPIII (Thomas et al., 2017). In this model, TRAPPIII activates Ypt1 at the Golgi and during autophagy. Thus, Ypt1 might regulate multiple membrane trafficking events (Thomas et al., 2017). Ypt1 is an essential protein and only several mutant variants that impair distinct functions of the protein can be analyzed (Sclafani et al., 2010).

The function of Ypt1 seems to be conserved from yeast to mammals. The human homolog of Ypt1 is Rab1. Rab1 has two isoforms Rab1A and Rab1B that locate to the ER and the Golgi (Plutner et al. 1991; Saraste et al., 1995). Rab1 regulates membrane transport from the ER to the Golgi and between compartments of the Golgi (Nuoffer et al., 1994; Pind et al., 1994). Furthermore, Rab1 modulates the recruitment of COPI (Alvarez et al., 2003). Haubruck and colleagues (1989) replaced the yeast protein Ypt1 with the mouse protein Rab1A and showed that the mammalian homolog was able to replace the function of Ypt1 in yeast (although only by protein overexpression under the GAL10 promoter). In mammals, the formation of autophagososmes also depends -similar to yeast- on the Ypt1 homolog Rab1 (Zoppino et al., 2010). Rab1B localizes to mammalian Atg9 vesicles. Furthermore, Rab1B and the GTPase-deficient mutant of Rab1B co-localize with LC3, one of the mammalian Atg8 proteins. Co-localization of both proteins was increased upon starvation and silencing of Rab1B leads to decreased formation of autophagosomes (Zoppino et al., 2010).

In yeast, Ypt1 is known to interact with Atg11 in its GTP-bound form and this interaction seems to be important for PAS formation under growing conditions (Lipatova et al., 2012). The recruitment of Atg1 to the PAS also depends on Ypt1. 
An impaired function of Ypt1 (ypt1-2) decreases recruitment of Atg1 while overexpression leads to accumulation of Atg1 at the PAS (Wang et al., 2013). The binding of Ypt1 and Atg1 is direct and preferentially between Atg1 and the active, GTP-bound form of Ypt1. This led to the proposal that Ypt1 recruits Atg1 to Atg9 vesicles, which are needed for phagophore initiation (Wang et al., 2013). Atg1 may tether Atg9 vesicles to PAS membranes or to each other. The authors (Wang et al., 2013) assume that Ypt1 does not regulate the kinase activity of Atg1. The Atg1 kinase function is needed, in later steps of autophagy. This was shown by the formation of Atg9 vesicle clusters that form at the PAS when kinase activity of Atg1 is lost (Lipatova et al., 2012). Three GAP proteins are reported to stimulate the GTP hydrolysis of Ypt1: Gyp1, Gyp5 and Gyp8 in vivo (De Antoni et al., 2002; Du and Novick, 2001).

\subsubsection{Gyp1 is a GTPase-activating protein for Ypt1}

Gyp1 (GAP for Ypt protein 1) is a cis-Golgi GTPase-activating protein (GAP) for yeast Rab proteins (Fig. 2.2B). It is membrane-associated and localizes to the Golgi where it negatively regulates Ypt1 (Du and Novick, 2001; Thomas et al., 2017). GAPs trigger the hydrolysis of GTPases and thereby inactivate them. The known Rab GAPs in yeast seem to have overlapping substrate specificity. For example, Gyp1 acts nearly equal on the yeast Rab members Sec4, Ypt1, Ypt7 and Ypt51 in vitro (Albert et al., 1999; Du et al., 1998). These overlapping functions might be the reason why differentiation of GAP targets in yeast lysates is difficult. Besides Gyp1, the GAPs Gyp5 and Gyp8 were also reported to use Ypt1 as a substrate (De Antoni et al., 2002; Du and Novick, 2001). All three GAPs are located differently in the cell. Gyp5 is almost completely cytosolic, Gyp1 localizes to the Golgi and Gyp8 might be part of large protein complexes like the ER or the plasma membrane. Localization of Gyp8 is not clear up to now (De Antoni et al., 2002). Although there is a special distribution of the Gyp proteins, it is assumed that they can compensate each other's function, if one Gyp is absent. This might explain why there is no obvious phenotype in protein transport detectable if a single Gyp is absent.

The crystal structure of the GAP domain of Gyp1 has been published in 2000 (Rak et al., 2000). The catalytically active domain consists of 16 a-helices and has a $\mathrm{V}$-shape. There are six sequence segments that are moderately conserved 
among the Gyp proteins and form the catalytically active region. Among those six motifs, three motifs are highly conserved GYP fingerprint sequences (RxxxW, IXXDXxR and $Y x Q$ ), which are also helpful to identify possible members of the protein family. The Gyp5 protein has a size of $101.6 \mathrm{kDa}$ and its GYP domain has a C-terminal localization. Gyp8, with a size of $57.6 \mathrm{kDa}$, has an N-terminal GYP domain (De Antoni, 2002). Gyp1 has at least two arginines Arg286 (important for the structure of Gyp1) and Arg343 (important for its GAP activity) that are essential for the function of the protein (Rak et al., 2000). The exposed arginine residue (R343) in the IxxDxxR motif is critical for the GAP activity and termed arginine finger (Alberts et al., 1999; Rak et al., 2000). Together with a glutamine finger in the YXQ motif, Gyp1 accelerates the hydrolysis of GTP by a dual finger mechanism (Pan et al., 2006).

Most of the known yeast GAPs possess the conserved TBC (Tre-2, Bub2 and Cdc16) domain, which is important for the catalytic activity of the protein (Pan et al., 2006). The TBC domain of Gyp1 has been shown to bind to Rab1, the mammalian Ypt1 homolog, and to Rab33 (Pan et al., 2016). With the mammalian GAP TBC1D22A, Gyp1 shares $48 \%$ sequence identity and the catalytic residues in both proteins align closely (Tempel et al., 2008). So far, no interaction with Rab1 has been reported for this protein. In humans, TBC1D22A seems to be linked to recessive genetic epilepsy with febrile seizures plus (Belhedi et al., 2013) and has an altered gene expression profile in patients infected with the human immunodeficiency virus (Shapshak et al., 2011). TBC1D22A has not been linked to autophagy in mammals yet. 


\subsection{Aim of the thesis}

Autophagy is a conserved transport pathway. It is needed for the degradation of cytosolic components and organelles and enables the cell to adapt to changing environments. Various proteins contribute to the formation of the autophagsome, which engulfs and transports its cargo to the degradative compartment. A core component of the autophagic machinery is Atg8. This protein plays a role in elongation and closure of the autophagosome (Xie et al., 2008) and in cargo recognition (Noda et al., 2008). Atg8 has been identified as a putative interaction partner of Gyp1 by mass spectrometry analysis, prior to this study. Gyp1 is one of the GAPs of the Rab GTPase Ypt1 (De Antoni et al., 2002). These proteins are known for their important role in membrane traffic and autophagy (Pfeffer, 2017; Yang and Rosenwald, 2016).

The aim of this study was to verify the potential interaction of Gyp1 and Atg8 and to analyze the role of this interaction during autophagy in S. cerevisiae. Prior to this study, it was shown that Ypt1 plays a role in early steps of autophagy. Ypt1 is supposed to recruit Atg1 (Wang et al., 2013) and also to interact with Atg11 (Lipatova et al., 2012). One aim of the study was to analyze whether Gyp1 is the responsible GAP for Ypt1 during the Cvt-pathway.

Atg8 has a role in later steps of autophagy and regulates elongation and closure of the autophagosome. Thus the second aim of this study was to analyze a potential role of the Gyp1-Atg8 interaction in later steps of autophagy. 


\section{Materials and Methods}

\subsection{Materials}

\subsubsection{Yeast strains}

Table 3.1: Yeast strains used in this study

\begin{tabular}{|c|c|c|}
\hline $\begin{array}{l}\text { S. cerevisiae } \\
\text { strain }\end{array}$ & Genotype & Reference \\
\hline WCG4 & $\begin{array}{l}\text { WCG4a MAT a his 2-11,15 leu2 - } \\
3,112 \text { ura3 }\end{array}$ & Thumm et al., 1994 \\
\hline Sey 6210 & $\begin{array}{l}\text { Sey } 6210 \text { MAT a ura 3-52 leu 2- } \\
3,112 \text { his 3- } \Delta 200 \\
\text { lys 2-801 trp1- } \Delta 901 \text { suc } 2-\Delta 9 \text { mel } \\
\text { GAL }\end{array}$ & $\begin{array}{l}\text { G. F. von Mollard } \\
\text { (University Bielefeld) }\end{array}$ \\
\hline $\operatorname{atg} 1 \Delta$ & $\begin{array}{l}\text { WCG4a MAT a his 2-11,15 leu2 - } \\
3,112 \text { ura3 atg1 } \triangle: \text { KAN }\end{array}$ & Straub et al., 1997 \\
\hline $\operatorname{atg} 1 \Delta$ & $\begin{array}{l}\text { WCG4a MAT a his 2-11,15 leu2 - } \\
3,112 \text { ura3 atg1 } \Delta:: H I S\end{array}$ & This study \\
\hline $\operatorname{atg} 8 \Delta$ & $\begin{array}{l}\text { WCG4a MAT a his 2-11,15 leu2 - } \\
3,112 \text { ura3 atg8 } \triangle:: K A N\end{array}$ & $\begin{array}{l}\text { AG Thumm } \\
\text { (Goettingen) }\end{array}$ \\
\hline $\operatorname{atg} 32 \Delta$ & $\begin{array}{l}\text { WCG4a MAT a his 2-11,15 leu2 - } \\
3,112 \text { ura3 atg32A::natNT2 }\end{array}$ & $\begin{array}{l}\text { AG Thumm } \\
\text { (Goettingen) }\end{array}$ \\
\hline Gyp1-6xHA & $\begin{array}{l}\text { WCG4a MAT a his 2-11,15 leu2 - } \\
3,112 \text { ura3 GYP1-6xHA::natNT2 }\end{array}$ & $\begin{array}{l}\text { AG Thumm } \\
\text { (Goettingen) }\end{array}$ \\
\hline Gyp5-6xHA & $\begin{array}{l}\text { WCG4a MAT a his 2-11,15 leu2 - } \\
\text { 3,112 ura3 GYP5-6xHA::natNT2 }\end{array}$ & $\begin{array}{l}\text { AG Thumm } \\
\text { (Goettingen) }\end{array}$ \\
\hline Gyp8-6xHA & $\begin{array}{l}\text { WCG4a MAT a his 2-11,15 leu2- } \\
\text { 3,112 ura3 GYP8-6xHA::natNT2 }\end{array}$ & $\begin{array}{l}\text { AG Thumm } \\
\text { (Goettingen) }\end{array}$ \\
\hline gyp1s & $\begin{array}{l}\text { WCG4a MAT a his 2-11,15 leu2 - } \\
3,112 \text { ura3 gyp14::hphNT1 }\end{array}$ & $\begin{array}{l}\text { AG Thumm } \\
\text { (Goettingen) }\end{array}$ \\
\hline gyp1ム & $\begin{array}{l}\text { Sey } 6210 \text { MAT a ura 3-52 leu 2- } \\
3,112 \text { his } 3-\Delta 200 \\
\text { lys 2-801 trp1- } \Delta 901 \text { suc } 2-\Delta 9 \text { mel } \\
\text { GAL gyp } 1 \Delta:: h p h N T 1\end{array}$ & $\begin{array}{l}\text { AG Thumm } \\
\text { (Goettingen) }\end{array}$ \\
\hline gyp5 5 & $\begin{array}{l}\text { WCG4a MAT a his 2-11,15 leu2 - } \\
\text { 3,112 ura3 gyp54::natNT2 }\end{array}$ & $\begin{array}{l}\text { AG Thumm } \\
\text { (Goettingen) }\end{array}$ \\
\hline gyp8s & $\begin{array}{l}\text { WCG4a MAT a his 2-11,15 leu2 - } \\
3,112 \text { ura3 gyp } 8 \Delta:: K A N\end{array}$ & $\begin{array}{l}\text { AG Thumm } \\
\text { (Goettingen) }\end{array}$ \\
\hline gyp1 $1 \Delta$ atg $8 \Delta$ & 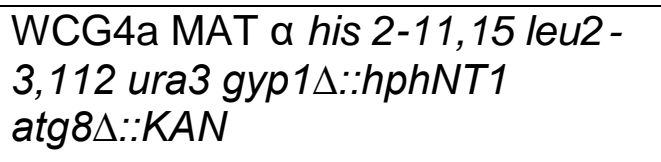 & $\begin{array}{l}\text { AG Thumm } \\
\text { (Goettingen) }\end{array}$ \\
\hline
\end{tabular}


WCG4a MAT a his 2-11,15 leu2 - AG Thumm

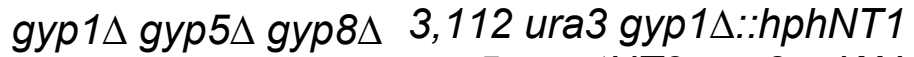
(Goettingen)

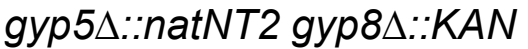

\begin{tabular}{|c|c|c|}
\hline gyp5 & 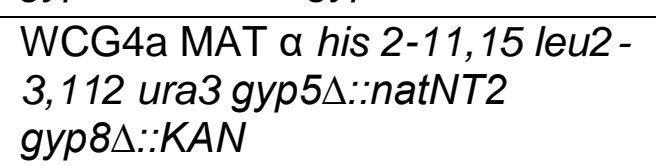 & $\begin{array}{l}\text { AG Thumm } \\
\text { (Goettingen) }\end{array}$ \\
\hline gyp1 & $\begin{array}{l}\text { WCG4a MAT a his } 2-11,15 \text { leu2 - } \\
3,112 \text { ura3 gyp } 1 \Delta:: \text { hphNT1 } \\
\text { gyp5s::natNT2 }\end{array}$ & $\begin{array}{l}\text { AG Thumm } \\
\text { (Goettingen) }\end{array}$ \\
\hline Atg14-GFP gyp1 $1 \Delta$ & $\begin{array}{l}\text { WCG4a MAT a his 2-11,15 leu2 - } \\
\text { 3,112 ura3 ATG14-GFP::HIS } \\
\text { gyp1 } 1:: \text { hphNT1 }\end{array}$ & $\begin{array}{l}\text { AG Thumm } \\
\text { (Goettingen) }\end{array}$ \\
\hline$g y p 1 \Delta \operatorname{atg} 1 \Delta$ & $\begin{array}{l}\text { WCG4a MAT a his } 2-11,15 \text { leu2 - } \\
3,112 \text { ura3 gyp } 1 \Delta:: \text { hphNT1 } \\
\operatorname{atg} 1 \Delta:: \text { HIS }\end{array}$ & This study \\
\hline Gyp1-6xHA & $\begin{array}{l}\text { WCG4a MAT } \alpha \text { his 2-11,15 leu2-- } \\
\text { 3,112 ura3 GYP1-6xHA::natNT2 }\end{array}$ & $\begin{array}{l}\text { AG Thumm } \\
\text { (Goettingen) }\end{array}$ \\
\hline Gyp5-6xHA & $\begin{array}{l}\text { WCG4a MAT a his 2-11,15 leu2 - } \\
\text { 3,112 ura3 GYP5-6xHA::natNT2 }\end{array}$ & $\begin{array}{l}\text { AG Thumm } \\
\text { (Goettingen) }\end{array}$ \\
\hline Gyp8-6xHA & $\begin{array}{l}\text { WCG4a MAT } \alpha \text { his 2-11,15 leu2-- } \\
\text { 3,112 ura3 GYP8-6xHA::natNT2 }\end{array}$ & $\begin{array}{l}\text { AG Thumm } \\
\text { (Goettingen) }\end{array}$ \\
\hline Gyp1-6xHA atg8 & $\begin{array}{l}\text { WCG4a MAT a his 2-11,15 leu2- } \\
3,112 \text { ura3 GYP1-6xHA::natNT2 } \\
\text { atg8 }:: \text { KAN }\end{array}$ & $\begin{array}{l}\text { AG Thumm } \\
\text { (Goettingen) }\end{array}$ \\
\hline Atg19-6xHA gyp1ム & $\begin{array}{l}\text { WCG4a MAT a his 2-11,15 leu2- } \\
\text { 3,112 ura3 ATG19-6xHA::natNT2 } \\
\text { gyp1A::hphNT1 }\end{array}$ & This study \\
\hline Atg14-GFP gyp1А & $\begin{array}{l}\text { WCG4a MAT a his 2-11,15 leu2 - } \\
\text { 3,112 ura3 ATG14-GFP::HIS } \\
\text { gyp1A::hphNT1 }\end{array}$ & $\begin{array}{l}\text { AG Thumm } \\
\text { (Goettingen) }\end{array}$ \\
\hline $\begin{array}{l}\operatorname{atg} 32 \Delta \text { pep } 4 \Delta \\
\text { gyp } 1 \Delta\end{array}$ & 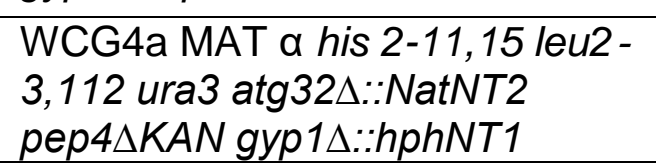 & This study \\
\hline $\begin{array}{l}\text { gyp } 1 \Delta \text { gyp } 5 \Delta \text { gyp } 8 \Delta \\
\text { atg } 8 \Delta\end{array}$ & $\begin{array}{l}\text { WCG4a MAT a his } 2-11,15 \text { leu2- } \\
3,112 \text { ura3 gyp } 1 \Delta:: \text { hphNT1 } \\
\text { gyp5 } 5:: \text { natNT2 gyp8 }:: \text { KAN } \\
\text { atg } 8 \Delta:: H I S\end{array}$ & This study \\
\hline
\end{tabular}




\subsubsection{E. coli strains}

Table 3.2: E. coli strains used in this study

\begin{tabular}{|c|c|c|}
\hline E. coli strain & Genotype & Reference \\
\hline $\mathrm{DH} 5 \alpha$ & $\begin{array}{l}F^{\prime}(\Phi 80 \text { ( } \triangle \text { lacZ) M15) } \Delta(\text { lacZYA- } \\
\text { argF) U169 recA1 endA1 hsdR17 } \\
r_{K}-m_{K}+\text { supE44 thi-1 gyrA relA }\end{array}$ & Hanahan, 1983 \\
\hline XL1 blue & $\begin{array}{l}\left.\text { endA1 gyrA96(nal }{ }^{R}\right) \text { thi-1 recA1 } \\
\text { relA1 lac glnV44 } F^{\prime}[:: T n 10 \text { proAB } \\
\text { lacla } \Delta\left(\text { lacZ)M15] hsdR17 }\left(r_{K^{-}} m_{K^{+}}\right)\right.\end{array}$ & Stratagene \\
\hline
\end{tabular}

\subsubsection{Plasmids}

Table 3.3: Plasmids used in this study

\begin{tabular}{|c|c|c|}
\hline Name & Genotype & Reference \\
\hline pRS313 & pRS313 CEN6 HIS3 cloning vector & Sikorski et al., 1989 \\
\hline pRS315 & pRS315 CEN6 LEU2 cloning vector & Sikorski et al., 1989 \\
\hline pRS316 & pRS316 CEN6 URA3 cloning vector & Sikorski et al., 1989 \\
\hline pFA6a-natNT2 & pFA6a-natNT2 & $\begin{array}{l}\text { Euroscarf (Janke et } \\
\text { al., 2004) }\end{array}$ \\
\hline pFA6a-HIS3MX6 & pFA6a-HIS3MX6 & $\begin{array}{l}\text { Longtine et al., } \\
1998\end{array}$ \\
\hline pUG23 & pUG23 CEN HIS3 & $\begin{array}{l}\text { AG Thumm } \\
\text { (Goettingen) }\end{array}$ \\
\hline pUG36 & $\begin{array}{l}\text { pUG36 CEN6 URA3 yEGFP3 N- } \\
\text { FUS }\end{array}$ & $\begin{array}{l}\text { AG Thumm } \\
\text { (Goettingen) }\end{array}$ \\
\hline MET25-Cherry & pUG36 CEN6 URA3 mCherry & Juris et al., 2015 \\
\hline Ape1-RFP & pRS315 CEN6 LEU2 Apel-RFP & $\begin{array}{l}\text { AG Thumm } \\
\text { (Goettingen) }\end{array}$ \\
\hline GFP-Atg8 & pRS316 CEN6 URA3 GFP-Atg8 & $\begin{array}{l}\text { AG Thumm } \\
\text { (Goettingen) }\end{array}$ \\
\hline GFP-Atg8 & pYES2 $2 \mu$ URA3 GAL-GFP-Atg8 & $\begin{array}{l}\text { AG Thumm } \\
\text { (Goettingen) }\end{array}$ \\
\hline GFP-Atg8 & pRS315 CEN6 LEU2 GFP-Atg8 & $\begin{array}{l}\text { AG Thumm } \\
\text { (Goettingen) }\end{array}$ \\
\hline GFP-Atg8_Y49A & $\begin{array}{l}\text { pYES2 } 2 \mu \text { URA3 GAL GFP-Atg8 } \\
\text { Y49A }\end{array}$ & $\begin{array}{l}\text { AG Thumm } \\
\text { (Goettingen) }\end{array}$ \\
\hline GFP-Atg8_L50A & $\begin{array}{l}\text { pYES2 } 2 \mu \text { URA3 GAL GFP-Atg8 } \\
\text { L50A }\end{array}$ & $\begin{array}{l}\text { AG Thumm } \\
\text { (Goettingen) }\end{array}$ \\
\hline GFP-Atg8_L50A & $\begin{array}{l}\text { pRS315 CEN6 LEU2 GFP- } \\
\text { Atg8_L50A }\end{array}$ & $\begin{array}{l}\text { AG Thumm } \\
\text { (Goettingen) }\end{array}$ \\
\hline GFP-Atg8_FK & $\begin{array}{l}\text { pYES2 } 2 \mu \text { URA3 GAL GFP-Atg8 } \\
\text { F5G K6G }\end{array}$ & $\begin{array}{l}\text { AG Thumm } \\
\text { (Goettingen) }\end{array}$ \\
\hline
\end{tabular}




\begin{tabular}{|c|c|c|}
\hline GFP-Atg8_ST & $\begin{array}{l}\text { pYES2 } 2 \mu \text { URA3 GAL GFP-Atg8 } \\
\text { S3AT4A }\end{array}$ & $\begin{array}{l}\text { AG Thumm } \\
\text { (Goettingen) }\end{array}$ \\
\hline 2xFlag-Atg8 & pRS313 CEN6 HIS3 2xFlag-Atg8 & This study \\
\hline Nui-Atg8 & pRS314 CEN6 TRP1 Niu-Atg8 & $\begin{array}{l}\text { AG Thumm } \\
\text { (Goettingen) }\end{array}$ \\
\hline GFP & pYES2 $2 \mu$ URA3 GAL GFP & $\begin{array}{l}\text { AG Thumm } \\
\text { (Goettingen) }\end{array}$ \\
\hline Atg1-GFP & pRS315 CEN LEU2 Atg1-GFP & Kraft et at., 2012 \\
\hline Gyp1 & pRS316 CEN6 URA3 Gyp1 & $\begin{array}{l}\text { AG Thumm } \\
\text { (Goettingen) }\end{array}$ \\
\hline Gyp1_AIM1 & $\begin{array}{l}\text { pRS316 CEN6 URA3 } \\
\text { Gyp1_W160A V164A }\end{array}$ & $\begin{array}{l}\text { AG Thumm } \\
\text { (Goettingen) }\end{array}$ \\
\hline Gyp1_R343K & pRS315 CEN6 LEU2 Gyp1_R343K & This study \\
\hline Gyp1_R343K & pRS316 CEN6 URA3 Gyp1_R343K & This study \\
\hline Gyp1_AIM1-6xHA & $\begin{array}{l}\text { pRS316 CEN6 URA3 Gyp1_- } \\
\text { W160A V164A-6xHA }\end{array}$ & $\begin{array}{l}\text { AG Thumm } \\
\text { (Goettingen) }\end{array}$ \\
\hline Gyp1_AIM2-6xHA & $\begin{array}{l}\text { pRS316 CEN6 URA3 Gyp1_F426A } \\
\text { L429A-6xHA }\end{array}$ & $\begin{array}{l}\text { AG Thumm } \\
\text { (Goettingen) }\end{array}$ \\
\hline Gyp1_AIM3-6xHA & $\begin{array}{l}\text { pRS316 CEN6 URA3 Gyp1_ } \\
\text { W625A L628A-6xHA }\end{array}$ & $\begin{array}{l}\text { AG Thumm } \\
\text { (Goettingen) }\end{array}$ \\
\hline Gyp1_AIM1-6xHA & $\begin{array}{l}\text { pRS315 CEN6 LEU2 Gyp1_W160A } \\
\text { V164A-6xHA }\end{array}$ & $\begin{array}{l}\text { AG Thumm } \\
\text { (Goettingen) }\end{array}$ \\
\hline Gyp1_AIM2-6xHA & $\begin{array}{l}\text { pRS315 CEN6 LEU2 Gyp1_F426A } \\
\text { L429A-6xHA }\end{array}$ & $\begin{array}{l}\text { AG Thumm } \\
\text { (Goettingen) }\end{array}$ \\
\hline Gyp1_AIM3-6xHA & $\begin{array}{l}\text { pRS315 CEN6 LEU2 Gyp1_W625A } \\
\text { L628A-6xHA }\end{array}$ & $\begin{array}{l}\text { AG Thumm } \\
\text { (Goettingen) }\end{array}$ \\
\hline Gyp1_AIM4-6xHA & $\begin{array}{l}\text { pRS315 CEN6 LEU2 Gyp1_F258A } \\
\text { I261A-6xHA }\end{array}$ & $\begin{array}{l}\text { AG Thumm } \\
\text { (Goettingen) }\end{array}$ \\
\hline Gyp1_AIM5-6xHA & $\begin{array}{l}\text { pRS315 CEN6 LEU2 Gyp1_W278A } \\
\text { I281A-6xHA }\end{array}$ & $\begin{array}{l}\text { AG Thumm } \\
\text { (Goettingen) }\end{array}$ \\
\hline Gyp1_AIM6-6xHA & $\begin{array}{l}\text { pRS315 CEN6 LEU2 Gyp1_W290A } \\
\text { L293A-6xHA }\end{array}$ & $\begin{array}{l}\text { AG Thumm } \\
\text { (Goettingen) }\end{array}$ \\
\hline Gyp1_AIM7-6xHA & $\begin{array}{l}\text { pRS315 CEN6 LEU2 Gyp1_W334A } \\
\text { I377A-6xHA }\end{array}$ & $\begin{array}{l}\text { AG Thumm } \\
\text { (Goettingen) }\end{array}$ \\
\hline Gyp1_R343K-6xHA & $\begin{array}{l}\text { pRS315 CEN6 LEU2 Gyp1_R343K- } \\
\text { 6xHA }\end{array}$ & This study \\
\hline Gyp1_R343K-6xHA & $\begin{array}{l}\text { pRS316 CEN6 URA3 } \\
\text { Gyp1_R343K-6xHA }\end{array}$ & This study \\
\hline Cherry-Atg11 & $\begin{array}{l}\text { pUG36 CEN6 URA3 mCherry- } \\
\text { Atg11 }\end{array}$ & $\begin{array}{l}\text { AG Thumm } \\
\text { (Goettingen) }\end{array}$ \\
\hline GFP-Atg32 & pUG36 CEN6 URA3 GFP-Atg32 & This study \\
\hline Ste14-Cub & $\begin{array}{l}\text { pRS313 CEN6 HIS3 MET25 Ste14- } \\
\text { Cub-RURA3 }\end{array}$ & $\begin{array}{l}\text { F. Reggiori } \\
\text { (University Medical } \\
\text { Center Utrecht) }\end{array}$ \\
\hline Nui-Ubc6 & $\begin{array}{l}\text { pRS314 CEN6 TRP1 CUP1 Nui- } \\
\text { Ubc6 }\end{array}$ & $\begin{array}{l}\text { AG Thumm } \\
\text { (Goettingen) }\end{array}$ \\
\hline
\end{tabular}




\begin{tabular}{lll} 
Atg19-Cub & $\begin{array}{l}\text { pRS313 CEN6 HIS3 MET25 Atg19- } \\
\text { Cub-RURA3 }\end{array}$ & $\begin{array}{l}\text { AG Thumm } \\
\text { (Goettingen) }\end{array}$ \\
\hline Cherry-Gyp1_AIM1 & mCherry-Gyp1_W160A V164A & This study \\
\hline $\begin{array}{l}\text { Cherry- } \\
\text { Gyp1_R343K }\end{array}$ & mCherry-Gyp1_R343K & This study \\
\hline mito-GFP & $\begin{array}{l}\text { pUG23 CEN6 HIS3 MET25 Suc9- } \\
\text { mtDHFR }\end{array}$ & $\begin{array}{l}\text { AG Thumm } \\
\text { (Goettingen) }\end{array}$
\end{tabular}

\subsubsection{Oligonucleotides}

Table 3.4: Oligonucleotides used in this study

\begin{tabular}{|c|c|}
\hline Name & Sequence ( $5^{\prime}$ to $\left.3^{\prime}\right)$ \\
\hline Cherry seq for & Ggcgcctacaacgtcaacatc \\
\hline pUG36 rev & gggacctagacttcaggttg \\
\hline GFP seq for & ggttctgttcaattagctgac \\
\hline hphNT1 r & CAATCGCGCATATGAAATCAC \\
\hline HIS 2r: & GCCTGGCGCTCCTGAACAG \\
\hline NatNT2 rev & CGATTCGTCGTCCGATTCGTC \\
\hline Atg8 seq Of & GGAGGCCGGTTATTTTCGG \\
\hline Atg8 seq $1 r$ & CTCTCCGACTCCGCCTTC \\
\hline Atg8 seq 1f & GAAGGCGGAGTCGGAGAG \\
\hline Atg8 seq $2 f$ & GGACGGGTTTTTGTATGTCAC \\
\hline Atg8 seq $2 r$ & GTGACATACAAAAACCCGTCC \\
\hline ATG8_KO-S2 & $\begin{array}{l}\text { CTATAATTTCGATTTTAGATGTTAACGCTTCATTTCTTT } \\
\text { TCATATAAAAGACTAATCGATGAATTCGAGCTCG }\end{array}$ \\
\hline ATG8_KO-S1 & $\begin{array}{l}\text { CTAATAATTGTAAAGTTGAGAAAATCATAATAAAAATA } \\
\text { ATTACTAGAGACATGCGTACGCTGCAGGTCGAC }\end{array}$ \\
\hline EcoR1-Atg8_fwd & AGTAGCAATgaattcCCGGAGGCCGGTTATTTTCGG \\
\hline 2xFlag-Atg8_r & $\begin{array}{l}\text { GACTTGGATCCCTTGTCGTCATCGTCTTTGTAGTCCT } \\
\text { TGTCGTCATCGTCTTTGTAG }\end{array}$ \\
\hline 2xFlag-Atg8_f & $\begin{array}{l}\text { GATGACGACAAGGACTACAAAGACGATGACGACAAG } \\
\text { GGATCCAAGTCTACATTTAAGTC }\end{array}$ \\
\hline GYP1_KO_hphNT1 & $\begin{array}{l}\text { CAACAACAACCACCAATACCGACCACTTAATAAAAGT } \\
\text { AACCATATAATGCGTACGCTGCAGGTCGAC }\end{array}$ \\
\hline GYP1_KO_hphNT1 & $\begin{array}{l}\text { GTTTTATATTATTACATACTATACAGTAAGTAAAATGA } \\
\text { ATAGGTCCGTTTAatcgatgaattcgagctcg }\end{array}$ \\
\hline GYP1_kr & CAGGTGTACCAAATCCACCG \\
\hline GYP1_kf & CACGATGAACTCGTTTAATGC \\
\hline Gyp1_forBamHI & tcaggatccATGGGTGTGAGATCCGC \\
\hline Gyp1_revSall & taggtcgacTTACAGCCAGTGCGACG \\
\hline $\begin{array}{l}\text { Gyp1_GFP_Sacl- } \\
\text { fwd }\end{array}$ & AGTAGGAATgagctcGGTGATCACTTAGGTAACAATC \\
\hline $\begin{array}{l}\text { Gyp1_GFP_HindIII- } \\
\text { rev }\end{array}$ & GGACAATTCAACGCGTCTGTG \\
\hline Gyp1_Sa & 1 \\
\hline
\end{tabular}




\begin{tabular}{|c|c|}
\hline cGYP1-HA-S2 (rev) & $\begin{array}{l}\text { GTTTTATATTATTACATACTATACAGTAAGTAAAATGA } \\
\text { ATAGGTCCGTTTAATCGATGAATTCGAGCTCG }\end{array}$ \\
\hline cGYP & CGAAGCCTTTATTTGGCAATCTCTTTACAAAGATGCTA \\
\hline & CGTCGCACTGGCTGCGTACGCTGCAGGTCGAC \\
\hline GYP1_AIM1. & $\begin{array}{l}\text { GACTTAGATGAGGATgcGAGTGCTGcTATTGACGATTA } \\
\text { TAATATGCC }\end{array}$ \\
\hline GYP1_Al & $\begin{array}{l}\text { GGCATATTATAATCGTCAATAgCAGCACTCgcATCCTC } \\
\text { ATCTAAGTC }\end{array}$ \\
\hline GYP1_AIM2 & $\begin{array}{l}\text { CAGATTTAGAAGCGGACACGgcTTGGTGTgcTACCAAA } \\
\text { TTGCTCGAAC }\end{array}$ \\
\hline GYP1_AIN & $\begin{array}{l}\text { GTTCGAGCAATTTGGTAgcACACCAAgcCGTGTCCGCT } \\
\text { TCTAAATCTG }\end{array}$ \\
\hline GYP1_AIM & $\begin{array}{l}\text { GCTTCTGAGCGAAGCCTTTATTgcGCAATCTgcTTACA } \\
\text { AAGATGCTACGTCG }\end{array}$ \\
\hline 1_Al & $\begin{array}{l}\text { CGACGTAGCATCTTTGTAAgcAGATTGCgcAATAAAGG } \\
\text { CTTCGCTCAGAAGC }\end{array}$ \\
\hline Gyp1_R3 & $\begin{array}{l}\text { GAGATAGATATACCGAagACAAATCCCCACATTCCCC } \\
\text { TC }\end{array}$ \\
\hline Gyp1_R3 & $\begin{array}{l}\text { GAGGGGAATGTGGGGATTTGTctTCGGTATATCTATCT } \\
\text { C }\end{array}$ \\
\hline P1_Se & CGGACAACCAGGCATATTAAGAC \\
\hline GYP1_Seq2for & CTCTAAGCACTGCAAGTAAC \\
\hline GYP1_Seq2rev & CAGCCAGTGCGACGTAGCATC \\
\hline P5_seq1f & CGATGACGAATCTGGTACTAC \\
\hline P5_seq2f & GGTCCTTCTGGACTCAAGTAG \\
\hline GYP5_seq3f & GGAAACCAGGATGACGCTTC \\
\hline GYP5_se & GCGCCTCTCTTTGTAGTTGG \\
\hline cGYP5-H & $\begin{array}{l}\text { CACAAATTGGAGAGGCACACAATGTGCACACAGCGC } \\
\text { AAAACTATACATCTAATCGATGAATTCGAGCTCG }\end{array}$ \\
\hline $\begin{array}{l}\text { cGYP5-HA-S3 } \\
\text { (fwd) }\end{array}$ & $\begin{array}{l}\text { CTAAGGCTAAAGGGAGGAAAGGCTGGACTGGTTTTA } \\
\text { AAAAAGTTTTTAAA CGTACGCTGCAGGTCGAC }\end{array}$ \\
\hline GYP5_KO_fw & $\begin{array}{l}\text { CAAAATAAAACAGCTCCTACCACCAGTGTAAAGTAGA } \\
\text { ACGTTAATAGAGCAATGCGTACGCTGCAGGTCGAC }\end{array}$ \\
\hline GYP5_KO_rev & $\begin{array}{l}\text { CACAAATTGGAGAGGCACACAATGTGCACACAGCGC } \\
\text { AAAACTATACATCTAatcgatgaattcgagctcg }\end{array}$ \\
\hline $\mathrm{P5} \_\mathrm{KO} \_\mathrm{kf}$ & GGATCGAACGTAATTGAGATTG \\
\hline P5_KO_kr & GGAAAGATTCTCTGCTGTTACG \\
\hline 3_ko_fwd & $\begin{array}{l}\text { GAGAAAATTTAGCAAATGACATAGAACAAGCCAATTA } \\
\text { ACAAAATACGATGcgtacgctgcaggtcgac }\end{array}$ \\
\hline gyp8_ko_rev & $\begin{array}{l}\text { ATATGAGTTTGTTTCTATGTCATGGGTATATAAGGTAT } \\
\text { ATTGTATGCTAatcgatgaattcgagctcg }\end{array}$ \\
\hline gyp8_seq1f & CTCTGGGAAGGGAAGATTATC \\
\hline $08 \_$seq2r & GACGCATTTTGCAATAGCAGG \\
\hline P8_seq1f & CGGGTATTGAGAAAGTACCC \\
\hline GYP8_seq2f & GCGAACAAACAAAGATGAACG \\
\hline cGYP & $\begin{array}{l}\text { CATATGAGTTTGTTTCTATGTCATGGGTATATAAGGTA } \\
\text { TATTGTATGCTAATCGATGAATTCGAGCTCG }\end{array}$ \\
\hline $\begin{array}{l}\text { CGYPE } \\
\text { (fwd) }\end{array}$ & $\begin{array}{l}\text { CTGGGTGGATCCGATTAGGGACATTTTGAAACTGGG } \\
\text { GCATCCAACTAGACGTACGCTGCAGGTCGAC }\end{array}$ \\
\hline
\end{tabular}




\begin{tabular}{ll} 
Atg1_kf & GGGGAAACAGAGAACAGTAC \\
\hline Atg1_kr & CATTGATTGATGACCTGCAAG \\
\hline atg1_ko_fw & $\begin{array}{l}\text { CCCCATATTTTCAAATCTCTTTTACAACACCAGACGAG } \\
\text { AAATTAAGAAAATGCGTACGCTGCAGGTCGAC }\end{array}$ \\
\hline atg1_ko_rev & $\begin{array}{l}\text { GCAGGTCATTTGTACTTAATAAGAAAACCATATTATGC } \\
\text { ATCACTTAATCGATGAATTCGAGCTCG }\end{array}$ \\
\hline pUG36- & AGTAGCAATgaattc ATGGTTTTGGAATACCAACAAAGG \\
\hline ATG32_Eco_f & ATTCCTACTTctcgagTTACAATAGAATATAACCCAGTG \\
\hline pUG36-ATG32_r & C \\
\hline Atg11-forBamHI & tcaggatcCATGGCAGACGCTGATGAATATAG \\
\hline Atg11-revXhol & TAGctcgagTCAAACTCCCTGGTATGAAACC
\end{tabular}

\subsubsection{Antibodies}

Table 3.5: Antibodies used in this study

\begin{tabular}{|c|c|c|}
\hline Antibody & Dilution & Source \\
\hline $\begin{array}{l}\text { anti-mouse- } \\
\text { HRPO-conjugate }\end{array}$ & $\begin{array}{l}\text { 1: } 10000 \text { in } 1 \% \\
\text { milk/TBST }\end{array}$ & Dianova, Hamburg \\
\hline $\begin{array}{l}\text { anti-rabbit- } \\
\text { HRPO-conjugate }\end{array}$ & $\begin{array}{l}\text { 1: } 5000 \text { in } 1 \% \\
\text { milk/TBST }\end{array}$ & Medac, Hamburg \\
\hline mouse-anti-GFP & $\begin{array}{l}\text { 1: } 2000 \text { in } 1 \% \\
\text { milk/TBST }\end{array}$ & Roche, Mannheim \\
\hline rabbit-anti-Ape1 & $\begin{array}{l}\text { 1: } 3000 \text { in } 1 \% \\
\text { milk/TBST }\end{array}$ & Eurogentech, Belgium \\
\hline mouse-anti-HA & $\begin{array}{l}\text { 1: } 10000 \text { in } \\
\text { TBST }\end{array}$ & Santa Cruz Biotechnology, Heidelberg \\
\hline mouse-anti-PGK & $\begin{array}{l}\text { 1: } 10.000 \text { in } 1 \% \\
\text { milk/TBST }\end{array}$ & Molecular Probes, Leiden, NL \\
\hline rabbit-anti-Ypt1 & $\begin{array}{l}\text { 1:1000 in } 1 \% \\
\text { milk/TBST }\end{array}$ & $\begin{array}{l}\text { Gift from Dr. Hans Dieter Schmitt (MPI for } \\
\text { Biophysical Chemistry, Goettingen) }\end{array}$ \\
\hline
\end{tabular}

\subsubsection{Commercial available kits}

Table 3.6: Commercial available kits used in this study

\begin{tabular}{ll} 
Name of the Kit & Source \\
\hline ECL Western Blotting Detection & Amersham Bioscience, GB \\
\hline NucleoSpin ${ }^{\circledR}$ Microbial DNA & Macherey-Nagel, Düren \\
\hline First Strand cDNA Synthesis Kit & Thermo Scientific \\
\hline QIAquick Gel Extraction Kit & Qiagen, Hilden \\
\hline QIAquick PCR Purification Kit & Qiagen, Hilden \\
\hline $\begin{array}{l}\text { QuikChange II Site-Directed } \\
\text { Mutagenesis Kit }\end{array}$ & Agilent Technologies, Santa Cruz, USA
\end{tabular}


QuikChange Lightning Site Directed Mutagenesis Kit

Wizard Plus SV Miniprep Kit
Agilent Technologies, Santa Cruz, USA

Promega, Hilden

\subsubsection{Chemicals, supplements, enzymes and protein purification systems}

Table 3.7: Chemicals, supplements, enzymes and protein purification systems used in this study

\begin{tabular}{|c|c|}
\hline Name of product & Source \\
\hline Bacto Agar & Becton Dickinson, Heidelberg \\
\hline Bacto Peptone & Becton Dickinson, Heidelberg \\
\hline Bacto Tryptone & Becton Dickinson, Heidelberg \\
\hline Bacto Yeast Extract & Becton Dickonson, Heidelberg \\
\hline Benzonase & Sigma, Deisenhofen \\
\hline clon NAT (nourseotricine) & Werner BioAgents, Jena \\
\hline $\begin{array}{l}\text { Complete }^{\mathrm{TM}} \text { protease inhibitor } \\
\text { (EDTA-free) }\end{array}$ & Roche, Mannheim \\
\hline $\begin{array}{l}\text { Deoxyadenosin-triphosphate } \\
\text { (dATP) }\end{array}$ & NEB, Frankfurt \\
\hline $\begin{array}{l}\text { Deoxycytidin-triphosphate } \\
\text { (dCTP) }\end{array}$ & NEB, Frankfurt \\
\hline $\begin{array}{l}\text { Deoxyguanosin-triphosphate } \\
\text { (dGTP) }\end{array}$ & NEB, Frankfurt \\
\hline $\begin{array}{l}\text { Deoxythymidin-triphosphate } \\
\text { (dTTP) }\end{array}$ & NEB, Frankfurt \\
\hline DNA-marker (1kb DNA-ladder) & NEB, Frankfurt \\
\hline ECL & USB, Santa Clara, CA \\
\hline GFP-TRAP & Chromotek, München \\
\hline Glass beads & Schütt, Goettingen \\
\hline Herring-sperm-DNA & Promega, Madison, USA \\
\hline Immersion oil & Applied Precision, USA \\
\hline Ligation buffer & NEB, Frankfurt \\
\hline $\begin{array}{l}\text { Difco yeast nitrogen base w/o } \\
\text { amino acids }\end{array}$ & Becton Dickinson, Heidelberg \\
\hline DNA polymerase (FideliTaq) & USB, Santa Clara, USA \\
\hline DNA polymerase (KOD) & Novagen, Darmstadt \\
\hline DNA polymerase (Taq) & NEB, Frankfurt \\
\hline DNA polymerase (Vent) & NEB, Frankfurt \\
\hline Phos-STOP & Roche \\
\hline $\begin{array}{l}\text { Precision Plus Protein All Blue } \\
\text { Standards }\end{array}$ & Biorad, München \\
\hline $\begin{array}{l}\text { Protease inhibitor cocktail } \\
\text { (bacteria) }\end{array}$ & Sigma, Deisenhofen \\
\hline RNAse A & Applichem, Darmstadt \\
\hline Restriction enzymes & NEB, Frankfurt \\
\hline
\end{tabular}


Skim milk powder

Granovita, Lüneburg

T4-Ligase NEB, Frankfurt

Difco Yeast nitrogen base w/o amino acids

Becton Dickinson, Heidelberg

Difco Yeast nitrogen base e/o amino acids and ammonium

Zymolyase T100

Becton Dickinson, Heidelberg

Seikagaku, Japan

\subsubsection{Equipment}

Table 3.8: Equipment used in this study

\begin{tabular}{|c|c|}
\hline Name of product & Source \\
\hline Agarose gel equipment & Bio-Rad Laboratories GmbH, München \\
\hline Bio RAD Mini-SUB Cell GT & Bio-Rad Laboratories GmbH, München \\
\hline Autoclave & $\begin{array}{l}\text { Adolf Wolf, SANOclav, Bad Überkingen- } \\
\text { Hausen }\end{array}$ \\
\hline Autoclave DX200 & Systec, Wettenberg \\
\hline Bench & $\begin{array}{l}\text { BDK Luft- und Reinraumtechnik } \mathrm{GmbH} \text {, } \\
\text { Sonnenbühl }\end{array}$ \\
\hline Blot Shaker GFL 3019 & GFL, Burgwedel \\
\hline Centrifuge 5804 & Eppendorf, Hamburg \\
\hline Centrifuge 5404R & Eppendorf, Hamburg \\
\hline Centrifuge 5415D & Eppendorf, Hamburg \\
\hline Centrifuge 5415R & Eppendorf, Hamburg \\
\hline Chemical balance & Sartorius, Goettingen \\
\hline Cuvettes no. 67.742 & Sarstedt, Nümbrecht \\
\hline $\begin{array}{l}\text { Cuvettes for electroporation; } \\
2 \mathrm{~mm}\end{array}$ & peqlab, Erlangen \\
\hline Electroporator 2510 & Eppendorf; Hamburg \\
\hline Freezer $\left(-20^{\circ} \mathrm{C}\right)$ & Liebherr, Bulle, $\mathrm{CH}$ \\
\hline Freezer $\left(-80^{\circ} \mathrm{C}\right)$ & Heareus, Hanau \\
\hline Glassbeads & Schütt, Goettingen \\
\hline Hood & $\begin{array}{l}\text { BDK Luft- und Reinraumtechnik, } \\
\text { Sonnenbrühl-Genkingen }\end{array}$ \\
\hline Incubator $\left(37^{\circ} \mathrm{C}\right)$ & Heraeus, Hanau \\
\hline Incubator 4200 & Innova, USA \\
\hline Incubator Thermomixer comfort & Eppendorf, Hamburg \\
\hline $\begin{array}{l}\text { Labshaker for diverse culture } \\
\text { sizes }\end{array}$ & A. Kühner, Birsfelden, Schweiz \\
\hline LAS 3000 Intelligent Dark Box & Fuji/Raytest, Benelux \\
\hline Magnetic stirrer MR 3001 & Heidolph, Kelheim \\
\hline $\begin{array}{l}\text { Microscope DeltaVision, } \\
\text { Olympus IX71 }\end{array}$ & Applied Precision, USA \\
\hline
\end{tabular}




\begin{tabular}{ll} 
Microscope slides (76x26mm) & Menzel-Gläser, Braunschweig \\
\hline Microscope cover slips & Menzel-Gläser, Braunschweig \\
\hline Microwave R-939 & Sharp, Hamburg \\
\hline $\begin{array}{l}\text { Multivortex IKA vibray VXR } \\
\text { basic }\end{array}$ & IKA, Staufen \\
\hline OmniTrays Nunc & SIGMA-ALDRICH, St. Louis, USA \\
\hline $\begin{array}{l}\text { Over head shaker Roto-Shake } \\
\text { Genie }\end{array}$ & Scientific Industries Inc, USA \\
\hline PCR Mastercycler gradient & Eppendorf, Hamburg \\
\hline pH meter pH537 & WTW, Weilheim \\
\hline Photometer & Eppendorf, Hamburg \\
\hline Pipettes & Eppendorf, Hamburg \\
\hline Pipette tips, petri dishes, ... & Sarstedt, Nümbrecht / Eppendorf, Hamburg \\
\hline PowerPac Basic Power Supply & Bio-Rad Laboratories GmbH, München \\
\hline PowerPac HC Power Supply & Bio-Rad Laboratories GmbH, München \\
\hline PVDF membrane Hybond-P & Amersham; GE healthcare, Freiburg \\
\hline Refrigerator (4 ${ }^{\circ}$ C) & Bosch, Stuttgart / Liebherr, Bulle, CH \\
\hline Rotor JA 10 & Beckmann, Krefeld \\
\hline Rotor JA 20 & Beckmann, Krefeld \\
\hline Rotor TLA-100.3 & Beckmann, Krefeld \\
\hline Rotor TLS-55 & Beckmann, Krefeld \\
\hline SDS-PAGE equipment BioRAD & Bio-Rad Laboratories GmbH, München \\
\hline Mini Protean cell & Whatman, GE healthcare, München \\
\hline Sterile filter & Eppendorf, Hamburg \\
\hline Thermomixer Comfort & Whatman Biometra, Goettingen \\
\hline Transilluminator TI 1 & Beckman, Krefeld \\
\hline Ultracentrifuge & Vacuubrand, Wertheim \\
\hline Vacuum pump & Thermo Electron, Karlsruhe \\
\hline Water bath SWB25 & Bio-Rad Laboratories GmbH, München \\
\hline Western Blot equipment & \\
\hline
\end{tabular}

\subsubsection{Media}

\subsubsection{YPD medium, pH 5.5}

YPD was used as a rich medium for yeast cells:

$1 \%$ Bacto $^{\circledR}$ Yeast Extract

$2 \%$ Bacto $^{\circledR}$ Pepton

$2 \%$ D-glucose 


\subsubsection{CM medium, pH 5.6}

Synthetic CM medium was used as yeast selection medium:

$0.67 \%$ Yeast Nitrogen Base w/o amino acids

$2 \%$ D-glucose*
$0.0117 \%$ L-alanine
$0.0117 \%$ L-methionine ${ }^{\star *}$
$0.0117 \%$ L-arginine
$0.0117 \%$ L-phenylalanine
$0.0117 \%$ L-asparagine
$0.0117 \%$ L-proline
$0.0117 \%$ L-aspartic acid
$0.0117 \%$ L-serine
$0.0117 \%$ L-cysteine
$0.0117 \%$ L-threonine
$0.0117 \%$ L-glutamine
$0.0117 \%$ tyrosine
$0.0117 \%$ L-glutamic acid
$0.0117 \%$ L-valine
$0.0117 \%$ L-glycine
$0.0117 \%$ myo-inositol
$0.0117 \%$ L-isoleucine
$0.0117 \%$ p-aminobenzoic acid

In dependence on selection conditions, the following supplements were added:
$0.3 \mathrm{mM}$ L-histidine
$0.3 \mathrm{mM}$ adenine
0.4 mM L-tryptophan
$1 \mathrm{mM}$ L-lysine
1.7 mM L-leucine
$0.2 \mathrm{mM}$ uracil

*In dependence on the intended growing condition (e.g. mitophagy) the carbon source D-glucose was replaced by $2 \%$ lactate or $2 \%$ galactose

**For overexpression experiments using a MET25 promoter L-methionine was excluded from the drop out mix.

\subsubsection{SD(-N) medium}

For starvation conditions of yeast, nitrogen free $\mathrm{SD}(-\mathrm{N})$ medium was used:

$0.67 \%$ Yeast Nitrogen Base w/o amino acids and w/o ammonium sulfate $2 \%$ D-glucose

\subsubsection{LB medium, pH 7.5}

For standard growth of $E$. coli cultures, LB-medium was used:

$1.0 \%$ Bacto $^{\circledR}$ Trypton

$0.5 \%$ Bacto $^{\circledR}$ Yeast extract

$0.5 \%$ sodium chloride 
For plasmid selection, $75 \mu \mathrm{g} / \mathrm{ml}$ ampicillin, $50 \mu \mathrm{g} / \mathrm{ml}$ kanamycin and/or $25 \mu \mathrm{g} / \mathrm{ml}$ chloramphenicol was added.

\subsubsection{SOC medium, pH 7.5}

SOC-medium was used during transformation of $E$. coli for cell regeneration:

$2 \%$ Bacto $^{\circledR}$ Trypton

$0.5 \%$ Bacto $^{\circledR}$ Yeast extract

$0.4 \%$ D-glucose

$10 \mathrm{mM}$ sodium chloride

$10 \mathrm{mM}$ magnesium sulfate

$10 \mathrm{mM}$ magnesium chloride

$2.5 \mathrm{mM}$ potassium chloride

\subsection{Methods}

\subsubsection{Cultivation and storage of S. cerevisiae}

For long-term storage of S. cerevisiae strains, a cryo-stock was prepared by using $30 \%$ sterile glycerol and yeast liquid culture (grown over night) in YPD medium in a 1:1 (650 $\mu \mathrm{l}$ each) proportion. Cultures were stored at $-80{ }^{\circ} \mathrm{C}$. For short-term storage, S. cerevisiae strains were stored on YPD plates at $4{ }^{\circ} \mathrm{C}$.

A liquid culture was prepared by inoculating the yeast strain from an agar plate in YPD medium or selection medium. Liquid cultures were grown at $30{ }^{\circ} \mathrm{C}$ with 220 rpm shaking. YPD medium was used as nutrient-rich medium containing $2 \%$ glucose. Synthetic selection medium CM, lacking the amino acids for selection, was used for selection of genetic markers. Cells were transferred using a sterile toothpick. Depending on the experimental setup, the liquid culture was used as a preculture to obtain the main culture. The main culture is a dilution from the preculture and used to adjust the desired $\mathrm{OD}_{600}$ and growth phase for the experiment. Cultures were incubated overnight (12-14 h) if not indicated otherwise.

\subsubsection{Cultivation and storage of E. coli}

The E. coli cell lines XL1 blue and $\mathrm{DH} 5 \alpha$ were used for all standard procedures. The cells were grown in LB medium, containing the respective antibiotic for 
plasmid selection. The liquid cultures were incubated at $37{ }^{\circ} \mathrm{C}$ and $220 \mathrm{rpm}$, overnight. In LB medium or on agar plates, E. coli was stored for 3-5 weeks at $4{ }^{\circ} \mathrm{C}$. For long term storage, a liquid culture was mixed with $60 \%$ sterile glycerol in a 1:1 (650 $\mu$ l each) ratio and stored at $-80^{\circ} \mathrm{C}$.

\subsubsection{Molecular biological methods}

\subsubsection{Determination of cell density}

To determine the cell density in liquid cultures, photometry was used. The culture was diluted by a factor of 10 and the $\mathrm{OD}_{600}$ (optical density measured at a wavelength of $600 \mathrm{~nm}$ ) was measured. A sample of the corresponding medium was used as a reference value. $1 \mathrm{OD}_{600}$ corresponds to $3 \times 10^{7}$ cells per $\mathrm{ml}$.

\subsubsection{Preparation of electrocompetent E. coli cells}

For the production of electrocompetent cells, $1 \mathrm{~L}$ of a main culture was incubated overnight in LB-medium. After reaching OD 600 0.5, cells were incubated for $10 \mathrm{~min}$ on ice, until they cooled down. Cells were pelleted for $8 \mathrm{~min}, 6500 \mathrm{rpm}$ at $4{ }^{\circ} \mathrm{C}$ and washed twice with ice-cold $\mathrm{dd}_{2} \mathrm{O}$ and once with ice-cold $10 \%(\mathrm{v} / \mathrm{v})$ glycerol. The pellet was resuspended in $2 \mathrm{ml}$ glycerol $(10 \%(\mathrm{v} / \mathrm{v}))$ and stored at $-80{ }^{\circ} \mathrm{C}$ in $40 \mu$ l aliquots.

\subsubsection{Determination of the DNA concentration}

The DNA concentration was determined by using the UV spectrophotometer (GE Healthcare). The amount of ultraviolet radiation absorbed by the bases was measured.

\subsubsection{Chromosomal DNA extraction from yeast cells}

For the isolation of the chromosomal DNA, $2 \mathrm{ml}$ of an overnight culture was

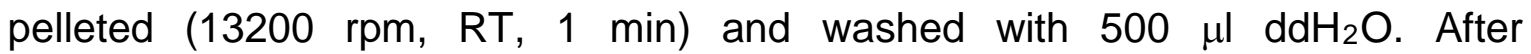
resupending the pellet in $200 \mu$ breaking buffer $(10 \mathrm{mM}$ Tris/ $\mathrm{HCl}, 100 \mathrm{mM} \mathrm{NaCl}, 1$ mM EDTA, $1 \%$ SDS, 2 \% Triton X-100), $200 \mu$ glass beads and $200 \mu \mathrm{l}$ phenol/chlorophorm were added. The compounds were mixed four times by alternating $1 \mathrm{~min}$ vortexing and $1 \mathrm{~min}$ incubation on ice. Finally $200 \mu \mathrm{ddH}_{2} \mathrm{O}$ were added. To remove cell debris and glass beads, the suspension was 
centrifuged for $10 \mathrm{~min}$ (RT, $13200 \mathrm{rpm}$ ), $300 \mu \mathrm{l}$ of the DNA-containing phase was transferred into a new cup and $1 \mathrm{ml}$ of $100 \%$ ethanol was added for precipitation of the DNA. Then the samples were incubated at $-20{ }^{\circ} \mathrm{C}$ for $10 \mathrm{~min}$. The supernatant was removed after a centrifugation step (13200 rpm, $4{ }^{\circ} \mathrm{C}, 10 \mathrm{~min}$ ) and incubated with $400 \mu \mathrm{lddH_{2 }} \mathrm{O}$ and $3 \mu \mathrm{l}$ RNase $\mathrm{A}(10 \mathrm{mg} / \mathrm{ml})$ at $37^{\circ} \mathrm{C}$ for $5 \mathrm{~min}$. After that a second precipitation step was performed. Therefore $1 \mathrm{ml}$ ice-cold ethanol (100\%) and $10 \mu \mathrm{l} 5 \mathrm{M}$ ammonium acetate were added and incubated at $20{ }^{\circ} \mathrm{C}$ for $20 \mathrm{~min}$. The DNA was pelleted (13200 rpm, RT, $10 \mathrm{~min}$ ) and resuspended in $30 \mu \mathrm{lddH_{2 }}$ O. Furthermore, the NucleoSpin ${ }^{\circledR}$ Microbial DNA (Macherey-Nagel) was used, according to the manufracturers instructions.

\subsubsection{Polymerase chain reaction (PCR)}

The polymerase chain reaction (PCR) was used to amplify DNA fragments for homologous recombination in yeast and the control of knockout strains. Two DNA oligomers (primers) that correspond to the complementary target region and flank the region of interest were designed. The PCR can be divided into 4 steps: initialization, denaturation, annealing and elongation. During cycles of repeated heating and cooling, the DNA was amplified by a DNA Polymerase. Depending on the needed accuracy and the length of the DNA product, different polymerases were used. Taq Polymerase was used for control PCRs (20 $\mu \mathrm{l}$ scale) and the KOD Hot Start DNA Polymerase for longer PCR fragments (50 $\mu \mathrm{l}$ scale). Genomic yeast DNA or plasmid DNA were used as template. The thermocycler program was adjusted to the size of the resulting DNA product (elongation time) and the annealing temperature of the primers.

\subsubsection{DNA agarose gel electrophoresis}

The DNA gel electrophoresis was used for analyzing of DNA fragments by size. Negatively charged DNA moves along the electric field to the anode. DNA samples were mixed with $10 \times$ sample buffer. Agarose gels consisted of $0.8 \%$ agarose in TAE buffer (40 mM Tris/acetate pH 8.2, 2 M EDTA, $0.144 \%$ acidic acid) and $1 \mu \mathrm{g} / \mathrm{ml}$ ethidium bromide for visualization of DNA by UV light. The DNA ladder Tri Dye 1kb (NEB) was used as a reference for size determination. 


\subsubsection{DNA gel extraction}

Using the "Qiagen Gel Extraction Kit" (Qiagen), DNA was purified after agarose gel electrophoreses. The kit was used according to manufactures advice.

\subsubsection{Restriction of DNA}

For the restriction of DNA, enzymes and buffers from NEB were used according to manufactures recommendations.

\subsubsection{Ligation of DNA fragments}

To ligate DNA fragments into a linearized vector, the T4-DNA-ligase (NEB) was used. DNA and vector were both prepared by DNA restriction and DNA gel extraction. In a total volume of $10 \mu \mathrm{l}, 1 \mu \mathrm{l}$ T4-ligase, $5 \mu \mathrm{l}$ DNA and $1 \mu$ l vector were incubated for $2 \mathrm{~h}$ at $25^{\circ} \mathrm{C}$ or at $16{ }^{\circ} \mathrm{C}$ overnight. The ligated vector was used for transformation in E. coli cells. The insert/vector ratio was calculated by the equation:

mass insert $(n g)=\underline{5 \times \text { mass plasmid } x \text { length insert }(\mathrm{bp})}$

length plasmid (bp)

\subsubsection{DNA sequencing}

New DNA-plasmids were verified by sequencing. GATC Biotech, Konstanz or Sequlab Sequence Laboratories, Goettingen performed the sequencing. DNA and respective primers were diluted according to recommendations of the service provider.

\subsubsection{Molecular cloning}

Molecular cloning was used to integrate recombinant DNA fragments into host organisms to direct their replication. By using PCR, the respective DNA fragment with two flanking restriction sites was generated. Accuracy of the PCR product was controlled by DNA electrophoresis and the respective product was purified using the gel extraction kit (Qiagen). The target plasmid together with the PCR product was then incubated with the respective restriction enzymes to generate restriction sites. Following the instruction protocol from the manufactures, a $50 \mu \mathrm{l}$ reaction was prepared and incubated for 2-3 hours at the recommended 
temperature. $1 \mu \mathrm{l} \mathrm{CIP} \mathrm{(alkaline} \mathrm{phosphatase,} \mathrm{calf} \mathrm{intestinal,} \mathrm{NEB)} \mathrm{was} \mathrm{added} \mathrm{to}$ dephosphorylate $5^{\prime}$ and $3^{\prime}$ ends of the vectors DNA to avoid religation. After purification of the reaction, the oligomers were verified by an agarose gel and purified using the gel extraction kit. For ligation, usually $0.5 \mu \mathrm{l}$ of T4-DNA-ligase, the PCR product and the target vector (10 $\mu$ l overall reaction volume) were incubated according to the manufacturers recommendation (NEB). The constructed plasmid was then transformed into electrocompetent cells.

\subsubsection{Site-directed mutagenesis of plasmids}

For site-directed mutagenesis of plasmids, the "QuikChange Lightning SiteDirected Mutagenesis Kit" (Aglient) was used. By constructing two complementary primers with exchanged nucleotides, desired point mutations were integrated into a DNA sequence. The kit was used according to the manufacturer's instructions. The introduced point mutations were verified by sequencing.

\subsubsection{Plasmid purification form E. coli}

For isolation of plasmid-DNA, the "Wizard Plus SV Kit" from Promega was used. E. coli cells were grown overnight in a liquid culture. The kit was used according to the manufacturer's instructions.

\subsubsection{Plasmid construction}

\subsection{2xFlag-Atg8 construct}

For pRS313-2xFlag-Atg8, Atg8 was amplified by the primers 2xFlag-Atg8_f and 2xFlag-Atg8_r using the overlapping extension PCR protocol. Plasmid DNA served as template. The resulting PCR product was cut with EcoRI and Xhol and ligated into pRS313.

\subsection{Gyp1 constructs}

For construction of pRS315-Gyp1_R343K, plasmid DNA was amplified using the primers Gyp1_R343K-fwd and Gyp1_R343K-rev. PCR was performed according to the QuikChange Lightning Site-Directed mutagenesis protocol. For construction of Cherry-Gyp1 (monomeric Cherry, hereafter Cherry), Gyp1 was amplified by PCR from chromosomal DNA using the primers Gyp1_forBamHI and 
Gyp1_revSall. The construct was ligated into pUG34-MET25_mCherry by BamHI and Sall. For construction of Cherry-Gyp1_R343K, Cherry-Gyp1 plasmid DNA was amplified using the primers Gyp1_R343K-fwd and Gyp1_R343K-rev. Mutagenesis was performed according to the QuikChange Lightning Site-Directed mutagenesis protocol.

\subsubsection{GFP-Atg32 construct}

Atg32 was amplified from plasmid DNA using the primers pUG36-Atg36_Eco_f and pUG36-Atg32_r. The PCR was performed according to the molecular cloning protocol. PCR product and plasmid were digested using the enzymes EcoRI and Xhol and ligated with the pUG36 vector.

\subsubsection{Yeast cell transformation with high efficiency}

The high efficiency transformation was used for chromosomal modifications of the yeast cells like gene knockouts or tagging of proteins. For this, $50 \mathrm{ml}$ YPD medium were inoculated with a yeast preculture grown to log phase. When the cells reached an $\mathrm{OD}_{600}$ of about 0.5 , they were diluted 1:10 with fresh YPD medium and incubated again until reaching an $\mathrm{OD}_{600}$ of 0.5 . Then the cells were

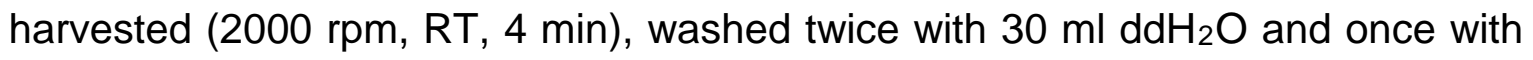
$2.5 \mathrm{ml}$ LiOAc-Sorb (100 mM lithium acetate, $10 \mathrm{mM}$ Tris, $1 \mathrm{mM}$ EDTA, $1 \mathrm{M}$ Dsorbitol, pH 8). After washing, the cell pellet was resuspended in 30-100 $\mu$ LiOAcSorb and incubated at $30{ }^{\circ} \mathrm{C}$ for $15 \mathrm{~min}$. After incubation, 40-100 $\mu \mathrm{l}$ cell suspension was incubated with $300 \mu \mathrm{l}$ PEG in Li-TE-buffer $(100 \mathrm{mM}$ lithium acetate, $10 \mathrm{mM}$ Tris/acetate $\mathrm{pH}$ 8.0, 1 mM EDTA, 40 \% PEG 3350), $5 \mu$ herringsperm DNA and $10 \mu \mathrm{l}$ of the respective DNA at $30^{\circ} \mathrm{C}$ for $30 \mathrm{~min}$ and at $42{ }^{\circ} \mathrm{C}$ for $15 \mathrm{~min}$. Cells were centrifuged (2000 rpm, RT, $5 \mathrm{~min}$ ) and resuspended in fresh YPD medium for recovery. After $2-3 \mathrm{~h}$ of incubation at $30{ }^{\circ} \mathrm{C}$, the cells were spread on an agar plate containing YPD and the respective selection.

\subsubsection{6 "Quick and dirty" transformation}

The "quick and dirty" transformation method was used to introduce plasmids isolated form E. coli into yeast. Therefore, $300 \mu \mathrm{l}$ PEG Li-TE-buffer $(100 \mathrm{mM}$ lithium acetate, $10 \mathrm{mM}$ Tris/acetate pH 8.0, 1 mM EDTA, 40 \% PEG 3350), $5 \mu \mathrm{l}$ 
herring-sperm DNA and 3-5 $\mu$ of the respective plasmid DNA were incubated at $30{ }^{\circ} \mathrm{C}$ for $30 \mathrm{~min}$ and at $42{ }^{\circ} \mathrm{C}$ for $15 \mathrm{~min}$. Cells were centrifuged (2000 rpm, RT, $5 \mathrm{~min}$ ), resuspended in $40-60 \mu \mathrm{ddH}_{2} \mathrm{O}$ and spread on a selection plate specific for the plasmid.

\subsubsection{Construction of knock out strains}

Deletion strains and chromosomal tagging were performed according to the protocols of Janke et al. (2004). For this, primers were designed consisting of a $\sim 45 \mathrm{bp}$ region homologous to the flanking region of the target gene and a $20 \mathrm{bp}$ region homologous to the selection gene. The plasmid containing the selection gene e.g. pFA6a-NatNT2, a nourseothricin cassette, was used as template for PCR amplification. The purified PCR product was then integrated by using the homologous recombination method (according to the protocol for high efficiency transformation of yeast). Thereby, the target gene was replaced with the selection gene or a chromosomal tag was integrated. After growth on selection plates, potential clones were verified by PCR.

\subsubsection{Direct fluorescence microscopy}

Direct fluorescence microscopy visualizes labeled proteins or plasmids in the cell. For this, the protein of interest is tagged either on the chromosome or on a plasmid with a protein that exhibits fluorescence when exposed to light in a defined range. The main cultures of the yeast strains were grown over night in the respective selection medium with or without addition of substances to enhance the amount or protein as for example methionine in a final concentration of 0.3 $\mathrm{mM}$ in case of a MET25 promoter.

The strain under investigation was dropped on a glass slide and covered by a cover slip. Analysis of the sample was performed using the DeltaVision Spectris fluorescence microscope (Olympus IX71, Applied Precision). Pictures were taken by a CoolSNAP HQ camera with the respective filter set for each fluorescent tag in the sample and an 100x objective. The yeast cell was sectioned into approximately 20 stacks, which were $0.2 \mu \mathrm{m}$ apart and placed in a way that covered the yeast cell from top to bottom. One fluorescent image was taken for each stack and one reference image from the middle of the sample. The obtained 
pictures were deconvoluted using the SoftWoRx software (Applied Science). Further editing and analyses were performed using Fiji and Illustrator software.

Table 3.9: Filter sets for live cell imaging

\begin{tabular}{l|l|l} 
Filter set & $\begin{array}{l}\text { Excitation wavelength } \\
(\mathbf{n m})\end{array}$ & $\begin{array}{l}\text { Emission wavelength } \\
(\mathbf{n m})\end{array}$ \\
\hline GFP & $475 / 28$ & $525 / 50$ \\
\hline mCherry & $575 / 25$ & $632 / 60$ \\
\hline Pol & $-50 / 28$ & $-50 / 0$
\end{tabular}

\subsubsection{Split-ubiquitin assay}

The Split-ubiquitin assay was used for the detection of protein-protein interactions. Yeast cells were transformed by using the vectors Cub and Nub. These vectors express the gene of interest, fused to either the N-terminal or the C-terminal half of ubiquitin (Müller and Johnsson, 2008). The Cub fragment was additionally attached to the Ura-3 reporter protein. Ura3 (orotidine 5-phosphate decarboxylase) is important for the synthesis of uracil. Ura3 converts 5Fluoroorotic acid (FOA) into 5-fluorouracil, which is toxic for the cell. This feature makes the Ura3 a positive and a negative reporter protein for the split-ubiquitin assay. If both proteins of interest interact with each other, the two parts of the ubiquitin protein can reassemble to create the ubiquitin protein. The reassemble leads to recognition of ubiquitin by ubiquitin specific proteases, which cleave the $\mathrm{R}$-Ura3p reporter. The free R-Ura3 protein exposes an $\mathrm{N}$-terminal (introduced) arginine residue, which leads to degradation of the protein according the $\mathrm{N}$-endrule and to uracil auxotrophy. Thus, cells expressing interacting proteins are able to grow on 5-FOA containing plates, but not on plates lacking uracil (Fig 3.1A). Cells expressing non-interacting proteins show the opposite growth behavior (Fig. 3.1B). 
A

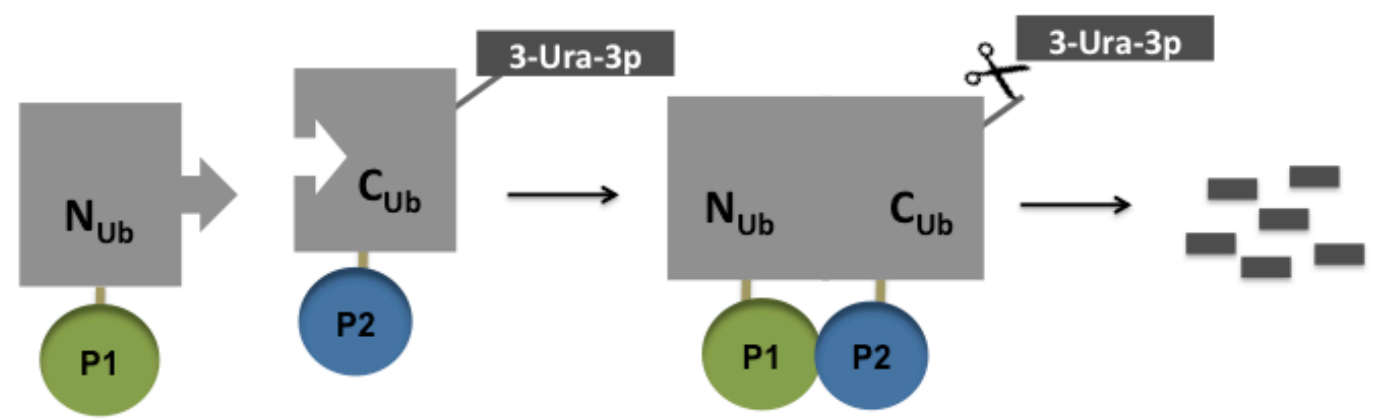

B

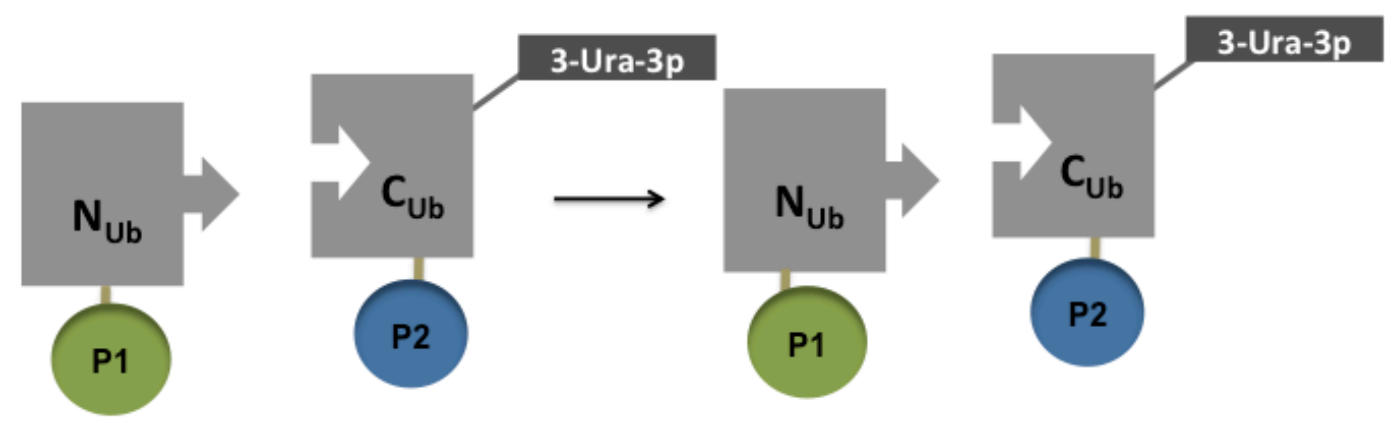

\section{Figure 3.1: The split-ubiquitin system}

(A) Two proteins of interest are fused to the $\mathrm{N}$-terminal and the C-terminal half of ubiquitin. Upon protein binding, the ubiquitin reassembles into a native-like protein that can be recognized by the ubiquitin-specific protease and a reporter protein R-Ura-3p, attached to the C-terminus of $\mathrm{C}_{\mathrm{Ub}}$, is cleaved. Thereby a C-terminal arginine is exposed and the R-Ura-3p is degraded. Cells expressing interacting $\mathrm{Nub} / \mathrm{C} \cup b$ fusion proteins are uracil auxotroph and resistant to 5-FOA.

(B) Cells expressing non-interacting proteins are able to grow in the absence of uracil but not in the presence of 5-FOA.

Based on a yeast overnight culture, a series of 10-fold dilutions from 10 to 10000 was prepared. The initial culture was set to $\mathrm{OD}_{600}=1.0$. From each dilution, $4 \mu \mathrm{l}$ were dropped on three solid plates. The first plate (CM w/o Trp and His) served as a control for growth of the strains and proper dilution. The FOA plate contained $\mathrm{CM}$ medium (w/o Trp and His), $250 \mu \mathrm{M}$ methionine, $100 \mu \mathrm{M} \mathrm{CuSO}_{4}$ and $1 \mathrm{mg} / \mathrm{ml}$ FOA, used for positive selection of plasmid interaction. The MV plate was made of the selection medium MV, lacking uracil (MV w/o Trp, His and Ura) and was used for negative selection of protein interaction. 


\subsubsection{Monitoring of pApe1 maturation}

The Cvt-pathway was monitored by maturation of pApe1 to mApe1 after its transport to the vacuole. For monitoring pApe1 maturation under nutrient-rich conditions, 2 OD 600 of a yeast culture with an OD600 between 2 and 3 were harvested and processed according to the alkaline lysis protocol. For Ape1 analysis under starvation conditions, cells were harvested (2000 rpm, RT, $4 \mathrm{~min}$ ), washed once with $2 \mathrm{ml} \mathrm{SD}-\mathrm{N}$ and then incubated in $2 \mathrm{ml} \mathrm{SD}-\mathrm{N}$, with a concentration of $10 \mathrm{OD}_{600}$ per ml. Samples were taken after 4 hours of incubation at $30{ }^{\circ} \mathrm{C}$, processed according to the alkaline lysis protocol and analyzed by immunoblotting. Ape1 was detected with Ape1 antibody.

\subsubsection{Induction and monitoring of mitophagy}

Cells expressing the mito-GFP marker Suc9-DHFR-GFP on a plasmid were grown in CM medium with $2 \%$ lactate and without methionine. Suc9-DHFR-GFP consists of the $N$. crassa subunit 9 of the $F_{1} F_{0}$ ATPase which is fused to mouse dihydrofolate reductase and GFP (Welter et al., 2013) and was expressed under a MET25 promoter. Mitophagy was monitored by the increase of free GFP over time as the mitochondria were degraded. The GFP tag is proteolytically resistant and can be observed by western blot analysis (Pfanner et al., 1987, Welter, 2011). For the observation of post-log mitophagy, a preculture was grown to an $\mathrm{OD}_{600}$ of $\approx 10$ (to transfer little as possible amounts of glucose to the main culture). Based on this preculture, a $10-15 \mathrm{ml}$ main culture was set to an OD600 of 0.5 or 0.75 . Samples were taken after 0, 24, 48 and $72 \mathrm{~h}$, processed according to the alkaline lysis protocol and analyzed by immunoblotting. To enhance protein separation, 6 $M$ urea was added to the seperation gel of the SDS-PAGE. For quantification of the immunoblots, the ratio of free GFP to mito-GFP was calculated.

\subsubsection{Biochemical methods}

\subsubsection{Alkaline lysis of yeast cells}

To analyze the protein composition in yeast cells, the alkaline lysis protocol was performed. 2 OD 600 of a cell culture were harvested (3000 rpm, RT, $5 \mathrm{~min}$ ) and

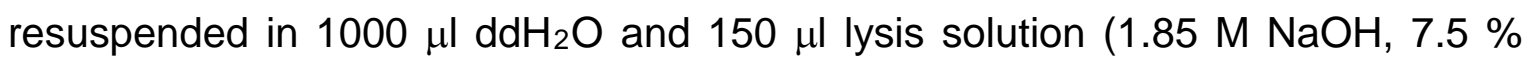


(v/v) B-mercaptoethanol). Samples were incubated on ice for $10 \mathrm{~min}$ and vortexted 4-6 times. Then, $150 \mu \mathrm{l}$ of a $50 \%$ TCA solution were added, samples were mixed and incubated on ice for $20 \mathrm{~min}$. To remove the lysis buffer, proteins were pelleted (13200 rpm, $10 \mathrm{~min}, 4^{\circ} \mathrm{C}$ ) and washed twice with $200 \mu \mathrm{l}$ acetone $\left(-20{ }^{\circ} \mathrm{C}\right)$. Then, samples were resuspended in $100 \mu \mathrm{l}$ SDS buffer $(116 \mathrm{mM}$ Tris/HCl pH 6.8, $12 \%$ (w/w) glycerol, $3.42 \%$ (w/v) SDS, $0.004 \%$ bromphenolblue, $2 \%$ ß-mercaptoethanol) and incubated on a shaker at $30{ }^{\circ} \mathrm{C}$ for 30 min. Samples were centrifuged (13200 rpm, RT, 2 min) and analyzed via SDSPAGE and immunoblotting.

\subsubsection{SDS-Polyacrylamide-Gel-Electrophoresis (SDS-PAGE)}

Using SDS-PAGE (sodium dodecyl sulfate polyacrylamide gelelectrophoresis) proteins were separated according to their electrophoretic mobility. Gels consisted of stacking (5\% acylamide) and separating (10/15 \% acylamide) parts. To enhance separation capacity of gels, $6 \mathrm{M}$ urea was added to the separation gel. This was done for the measurement of mitophagy. 10-15 $\mu$ sample was loaded on the gel as well as the All Blue Standard from Bio-Rad as reference. Electrophoresis was performed at $150 \mathrm{~V}$ using an electrophorese chamber (BioRad) and SDS page-running buffer (200 mM glycerol, $25 \mathrm{mM}$ Tris and $1 \%$ SDS in $\mathrm{H}_{2} \mathrm{O}$ ).

Table 3.10: Contents of SDS Polyacrylamid gel used in a Bio-Rad MiniProtean III electrophoresis chamber

\begin{tabular}{llll} 
Components & \multicolumn{2}{l}{ Separation Gel } & Stacking Gel \\
\hline & $10 \%$ PAA & $15 \%$ PAA & $5 \%$ PAA \\
\hline ddH2O & $1.9 \mathrm{ml}$ & $1.2 \mathrm{ml}$ & $3.0 \mathrm{ml}$ \\
\hline $1.5 \mathrm{M}$ Tris, $\mathrm{pH} 8.8$ & $1.25 \mathrm{ml}$ & $1.25 \mathrm{ml}$ & - \\
\hline $0.5 \mathrm{M}$ Tris, $\mathrm{pH} 6.8$ & - & - & $1.25 \mathrm{ml}$ \\
\hline Protogel & $1.8 \mathrm{ml}$ & $2.5 \mathrm{ml}$ & $1.8 \mathrm{ml}$ \\
\hline $10 \%(\mathrm{w} / \mathrm{v})$ SDS & $50 \mu \mathrm{l}$ & $100 \mu \mathrm{l}$ & $50 \mu \mathrm{l}$ \\
\hline $10 \%(w / v)$ APS & $50 \mu \mathrm{l}$ & $100 \mu \mathrm{l}$ & $50 \mu \mathrm{l}$ \\
\hline TEMED & $2.5 \mu \mathrm{l}$ & $5 \mu \mathrm{l}$ & $5 \mu \mathrm{l}$
\end{tabular}




\subsubsection{Immunoblotting}

To transfer proteins separated by SDS-PAGE from the polyacrylamide gel to a PVDF (Polyvinylidendifluorid) membrane, the immunoblotting (western blot) method was used. The wet-blot buffer consisted of $25 \mathrm{mM}$ Tris, $192 \mathrm{mM}$ glycine and $20 \%$ ethanol. The transfer was performed using a blotting chamber from BioRad, at $4{ }^{\circ} \mathrm{C}$ for at least $4 \mathrm{~h}$ or overnight. Each gel was blotted with $75 \mathrm{~mA}(1.2$ $\mathrm{mA}$ per $\mathrm{cm}^{2}$ ). The SDS gel and the membrane were placed between $2 \times 2$ Whatman papers and two sponge pads. Then the gel holder cassette was closed and placed into the chamber (Fig. 3.2).

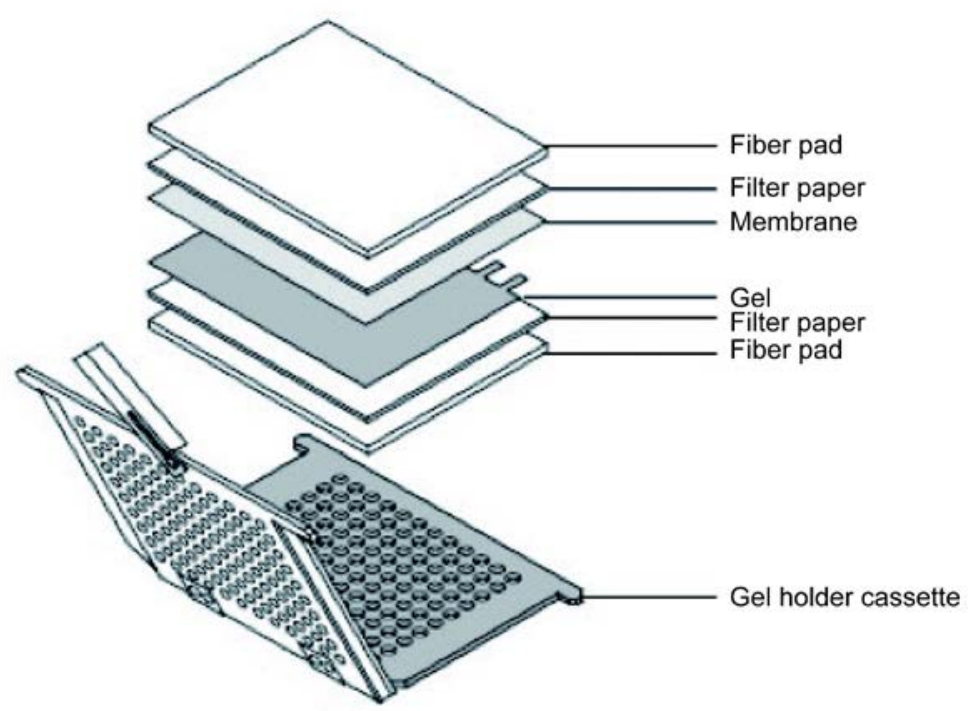

Figure 3.2: Immunoblot setting (adapted from Mini Trans-Blot ${ }^{\circledR}$ Electrophoretic Transfer Cell Instruction Manual)

By using the wet immunoblot, proteins are transferred from the polyacrylamide gel to a PVDF membrane that can be analyzed with the respective antibodies.

After the transfer, membranes were incubated in blocking solution (10\% (w/v) skim milk powder in TBST (20 mM Tris/HCl pH 7.6, $137 \mathrm{mM} \mathrm{NaCl}_{2}, 0.1 \%$ (w/v) Tween20) for $1 \mathrm{~h}$ at room temperature or at $4{ }^{\circ} \mathrm{C}$ overnight. Proteins in the milk powder thereby mask unspecific binding sites. To remove the blocking solution, the membrane was washed three times with $25 \mathrm{ml}$ TBST for $10 \mathrm{~min}$. Then, the primary antibody was added and incubated for at least $2 \mathrm{~h}$ at RT or at $4{ }^{\circ} \mathrm{C}$ overnight. The secondary antibody was diluted in TBST, optionally with $1 \%$ (w/v) skim milk powder, and incubated for $1 \mathrm{~h}$ on the washed membrane $(3 \times 10 \mathrm{~min}$, using $25 \mathrm{ml}$ TBST buffer). The membrane was analyzed using the $\mathrm{ECL}^{\mathrm{TM}}$ solution provided by USB. For the analysis, the membrane was washed 6 times ( $3 \mathrm{~min}$ ) with TBST and then incubated with the $\mathrm{ECL}^{\mathrm{TM}}$ solution for $4 \mathrm{~min}$. Signals were 
visualized using the LAS-3000 (Fujifilm). The AIDA software (Version 4.06.116) was used for further analyses and quantification. Removal of the antibodies was performed using $10 \%$ acetic acid for $10 \mathrm{~min}$. After washing and incubation in blocking solution, the membrane can be analyzed again with another antibody.

\subsubsection{GFP-TRAP}

The GFP-TRAP was used to detect protein-protein interactions in vivo. The bait protein was therefore expressed with a GFP tag that can attach to GFP beads via GFP binding proteins on the surface of the beads. Multiple washing steps then purified the beads together with the bound bait protein as well as interacting proteins or protein complexes.

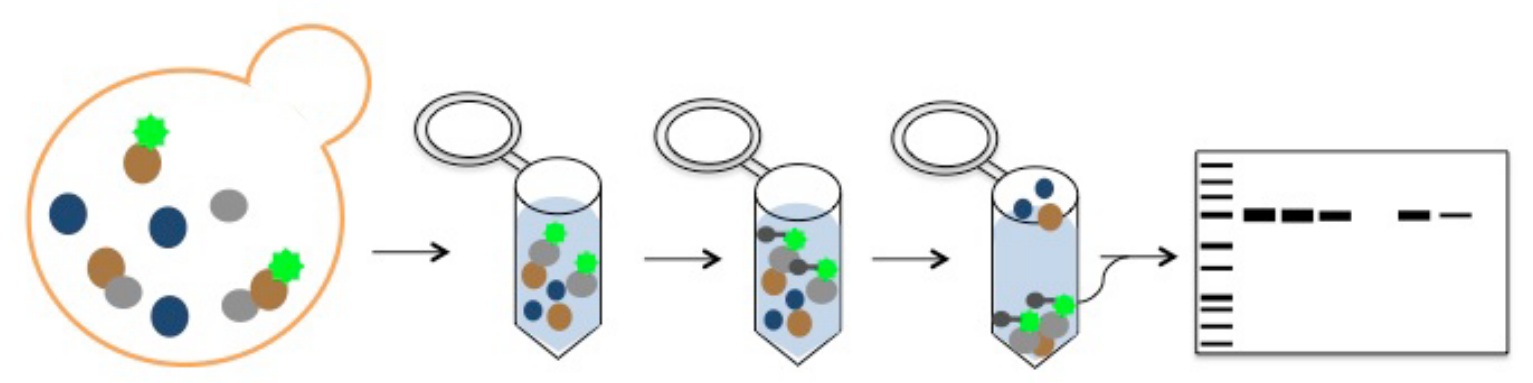

Figure 3.3: GFP-TRAP procedure

The GFP-TRAP approach was used to detect protein-protein interactions in vivo. For this, a bait protein was expressed with a GFP tag that is able to interact with the GFP-binding protein on the surface of the GFP-TRAP beads. The GFP-TRAP beads together with their interacting proteins are then purified and analyzed by immunoblot.

150 to $1000 \mathrm{OD}_{600}$ of cells were pelleted by centrifugation (2000 rpm, 5 min, 4 ${ }^{\circ} \mathrm{C}$ ), washed with $15 \mathrm{ml}$ cold PBS and resuspended in GFP-TRAP buffer. Cells were then incubated with $200 \mu \mathrm{l}$ glass beads (Schütt, Goettingen, Germany) on a shaker (Disruptor Genie ${ }^{\circledR}$ digital 230V, Schütt Labortechnik, Goettingen, Germany) for $30 \mathrm{~min}$. After opening, cells were centrifuged ( $5 \mathrm{~min}, 2700 \mathrm{xg}, 4^{\circ} \mathrm{C}$ ) to remove glass beads and cell debris, and the supernatant (Input) was incubated with GFP-TRAP beads (GFP-Trap_A, ChromoTek, Planegg-Martinsried, Germany) at $4{ }^{\circ} \mathrm{C}$ on an overhead shaker (Roto-Shake Genie, Scientific Industries Inc, USA). After $2 \mathrm{~h}$ incubation, the GFP-TRAP beads were washed 5-7 times using 600-800 $\mu$ l GFP-TRAP buffer (centrifugation step at $2000 \mathrm{xg}$ for $2 \mathrm{~min}$ between each washing step). Proteins were eluted in $25 \mu$ l GFP-TRAP buffer and 
$25 \mu \mathrm{l}$ 4x Laemmli. Resuspended beads were heated to $95^{\circ} \mathrm{C}$ for $10 \mathrm{~min}$ and 10 $25 \mu \mathrm{l}$ of the sample was loaded to a polyacrylamide gel and analyzed by immunoblotting.

Table 3.11: GFP-TRAP buffers

GFP-TRAP buffer PBS

\begin{tabular}{ll}
\hline PBS pH 7.4 & $1 \times$ \\
\hline Sorbitol & $0.2 \mathrm{M}$ \\
\hline MgCl2 & $5 \mathrm{mM}$ \\
\hline Triton X-100 & $1 \%$ \\
\hline Inhibitor Mix & $1 \times$ \\
\hline Complete EDTA-free & $1 \times$ \\
\hline PMSF & $1 \mathrm{mM}$
\end{tabular}

GFP-TRAP buffer Tris

\begin{tabular}{ll}
\hline Tris $-\mathrm{HCl} \mathrm{pH} 7.5$ & $50 \mathrm{mM}$ \\
\hline $\mathrm{NaCl}$ & $100 \mathrm{mM}$ \\
\hline Triton $\mathrm{X}-100$ & $0.2 \%$ \\
\hline Inhibitor Mix & $1 \mathrm{x}$ \\
\hline Complete EDTA-free & $1 \mathrm{x}$ \\
\hline PMSF & $1 \mathrm{mM}$ \\
\hline Phos-STOP & $1 \mathrm{x}$
\end{tabular}

Buffer conditions were adjusted according to the experimental setup. For the interaction studies of Atg8 and Gyp1 as well as Ypt1 and Atg1, GFP-TRAP buffer PBS was used. For analysis of Atg8 and Atg32, the GFP-TRAP buffer Tris was used. Depending on the protein abundance in the cell, the amount of harvested cells and GFP-TRAP beads was adjusted (Tab. 3.12). For western blot analysis, the input was diluted as indicated in table 3.12 (\% Input).

Table 3.12: GFP-TRAP conditions for different experimental setups

\begin{tabular}{lllll} 
Analyzed proteins & Promoter & $\begin{array}{l}\text { Cell amount } \\
\text { (OD600) }\end{array}$ & $\begin{array}{l}\text { GFP-TRAP } \\
\text { beads }\end{array}$ & Input \\
\hline Atg8 and Gyp1 & endogenous & 1000 & $5 \mu \mathrm{l}$ & 0.083 \\
\hline Atg8 and Gyp1 & pYES2 GAL & $150-250$ & $8 \mu \mathrm{l}$ & 2.5 \\
\hline Atg1 and Ypt1 & endogenous & 1000 & $5 \mu \mathrm{l}$ & 0.014 \\
\hline Atg8 and Atg32 & MET25 & $150-250$ & $5 \mu \mathrm{l}$ & 0.84
\end{tabular}




\subsubsection{Statistical analyses}

For the statitistical analysis of western blots and fluorescence microscopy the Graph Pad Prism 6 software was used. The error bars represent SEM (standard error of the mean) values. For fluorescence microscopy, pictures from at least 3 independent cultures were taken. The number of dots/cell was calculated from these images and analyszed by the unpaired two tailed t-tests. The number of pictures used for the calculation is indicated for each experiment. Statistical relevance is indicated as follows: not significant for $P>0.05$ (no asterisk), * for $P$ $<0.05,{ }^{* *}$ for $\mathrm{P}<0.01,{ }^{* *}$ for $\mathrm{P}<0.001$ and ${ }^{* \star * *}$ for $\mathrm{P}<0.0001$. Western blots were quantified using the AIDA software.

For the GFP-TRAP experiments, the ratio of bound fraction and input fraction of the co-preticipated protein was calculated and divided by the bound fraction of the bait. The WT was set to $100 \%$. The GFP-Atg32 protein was highly unstable and the bound fraction was not considered for the analysis. Western blots were furthermore analyzed for their statistical relevance. For this, a one sample t-test was used. 


\section{Results}

\subsection{Interaction analysis of Gyp1 and Atg8}

Atg8 is known to participate in various complexes during autophagy. To determine possible interaction partners that are functionally involved in those complexes, a mass spectrometry analysis was performed prior to this study. Recombinant Atg8 was tagged by GST and incubated with the crude extract of wild type (WT) yeast cells. Thereby, Gyp1 was identified as a potential interaction partner of Atg8. Gyp1 is a GTPase-activating enzyme for Ypt1 that has important functions in vesicle transport and compartment identity (Du and Novick, 2001; Pfeffer, 2017). Therefore, Gyp1 seemed to be a likely candidate that could be involved in Atg8complexes and was further analyzed.

\subsubsection{Atg8 preferentially interacts with Gyp1}

By using a GFP-TRAP approach, binding of Gyp1 and Atg8 was analyzed in vivo. The two GAPs Gyp5 and Gyp8 were included in many experiments as both proteins are reported to use Ypt1 as a substrate similar to Gyp1 (Lafourcade et al., 2004). GFP-ATG8 was expressed from a pYES2 plasmid/GAL promoter and the GAP proteins were chromosomally tagged by HA. Cells were grown to logarithmic phase before lysis and co-precipitation of the proteins. The proteins were detected in western blot analysis by GFP and HA antibody, respectively. Gyp1-HA and Gyp5-HA were present in almost equal protein amounts (Input) and the Gyp8-HA protein was slightly less abundant (Fig. 4.1A). Compared to Gyp1HA, co-precipitation of Gyp5-HA was not detectable in the bound fraction. The interaction of GFP-Atg8 and Gyp8-HA was only weak. All three GAPs did not bind to the GFP tag alone, indicating a specific interaction between Atg8 and Gyp1. To further analyze this experiment, the enrichment factor was calculated (bound value divided by input value). Comparing input and bound fraction of Gyp1-HA, an enrichment factor of $0.68 \pm 0.10$ was calculated. The enrichment factor was significantly lower for Gyp5-HA and Gyp8-HA with $0.01 \pm 0.01$ and $0.09 \pm 0.03$ (Fig. 4.1B). Thus, the interaction of Atg8 occured preferentially between Atg8 and Gyp1, was only weak for Atg8 and Gyp8 and hardly detectable for Atg8 and Gyp5. Next, the interaction sites of Atg8 and Gyp1 were analysed. 

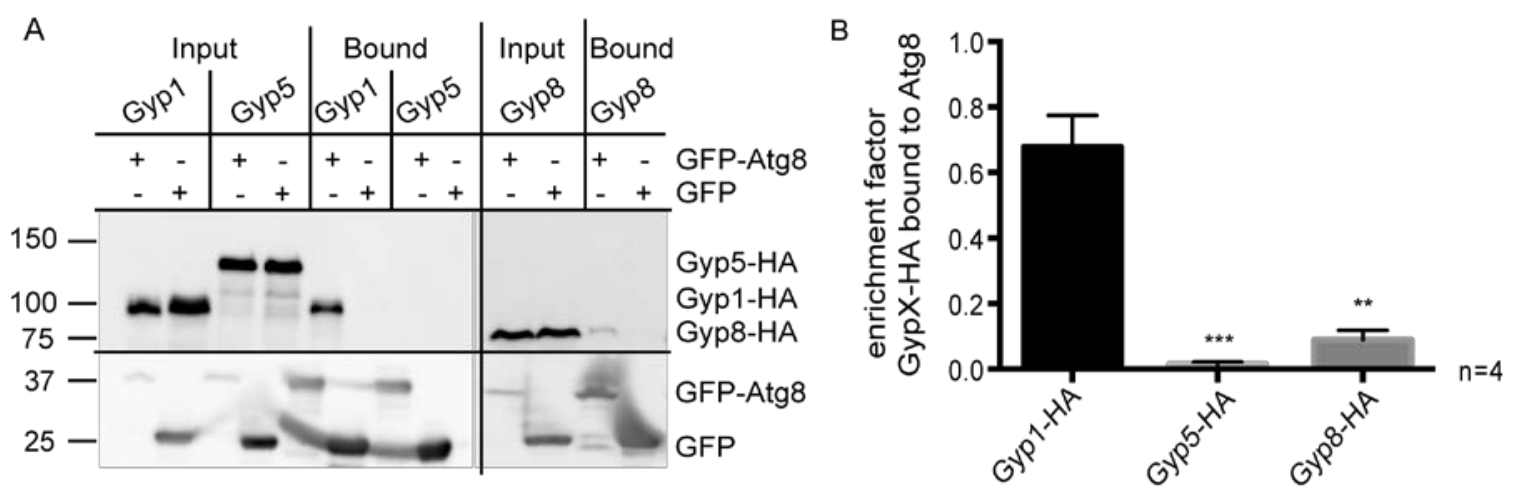

Figure 4.1: Atg8 interacts preferentially with Gyp1

(A) Western blot analysis of a GFP-TRAP approach show preferential binding of Atg8 and Gyp1. The experiments were performed after growing the cells to logarithmic growth phase. GFP-ATG8 was expressed from the GAL1 promoter on plasmid. GYP1, GYP5 and GYP8 were chromosomally tagged with HA. GFP-Atg8 was bound to the GFP-binding protein of the GFP-TRAP beads and Gyp1-HA was co-immunoprecipitated. GFP-Atg8 was detected by GFP antibody, Gyp1-HA by HA antibody. The molecular weight marker is depicted in kDa.

(B) Quantification and analyses of four independent GFP-TRAP experiments. The number of independent experiments is indicated by $\mathrm{n}$. The enrichment factor was calculated by dividing bound value by input value. Statistical analysis was performed using the one sample t-test, error bars represent SEM and asterisks represent $p$-values. Gyp1-HA is highly enriched in the GFP-TRAP bound fraction due to its binding to GFP-Atg8. Compared to Gyp1-HA, the interaction of GFP-Atg8 to Gyp5-HA and Gyp8-HA is significantly lower.

\subsubsection{Gyp1 interacts with the AIM binding sites of Atg8}

To further analyze and define the binding of Gyp1 and Atg8, potential binding sites in both proteins were identified and mutated. To identify a binding site for Gyp1 in Atg8, one residue in each hydrophobic pocket and two residues in the $\mathrm{N}$ terminal helical domain (NHD) of Atg8 were mutated. The hydrophobic pockets in Atg8 are composed of defined side chains and are required for binding of AIMs (Chapter 2.5.6). The Atg8_L50A mutation is located in the W-site, the Atg8_Y49A mutation in the L-site of the hydrophobic pockets in Atg8. The FK mutation (F5G K6G) as well as the ST mutation (S3A T4A) are located in the NHD of Atg8. The ST mutant served as a negative control, as this region is not conserved and no binding has been reported. 
A

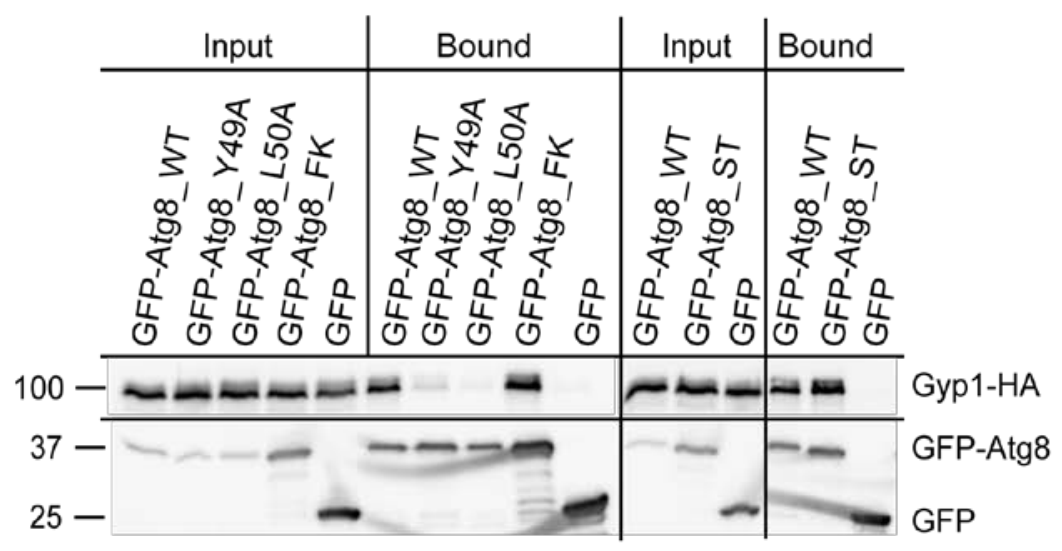

B

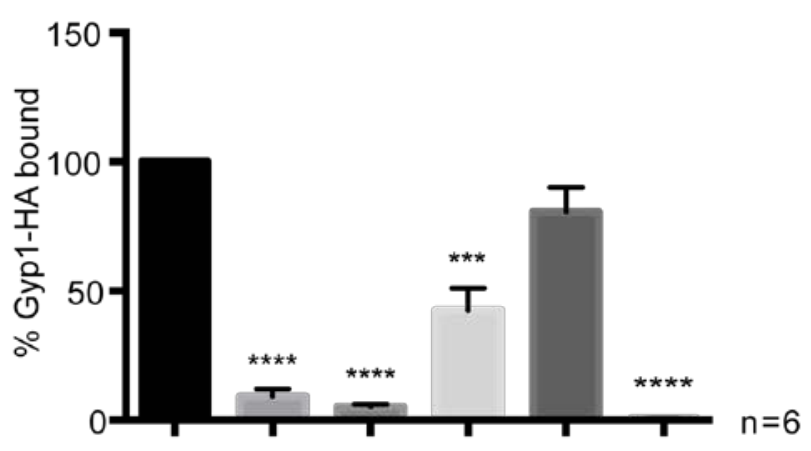

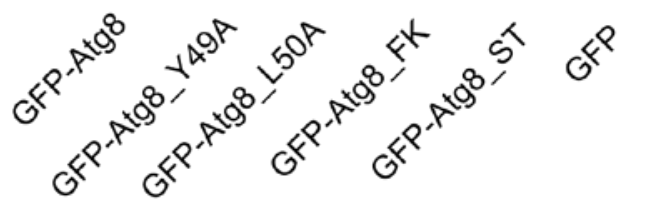

C
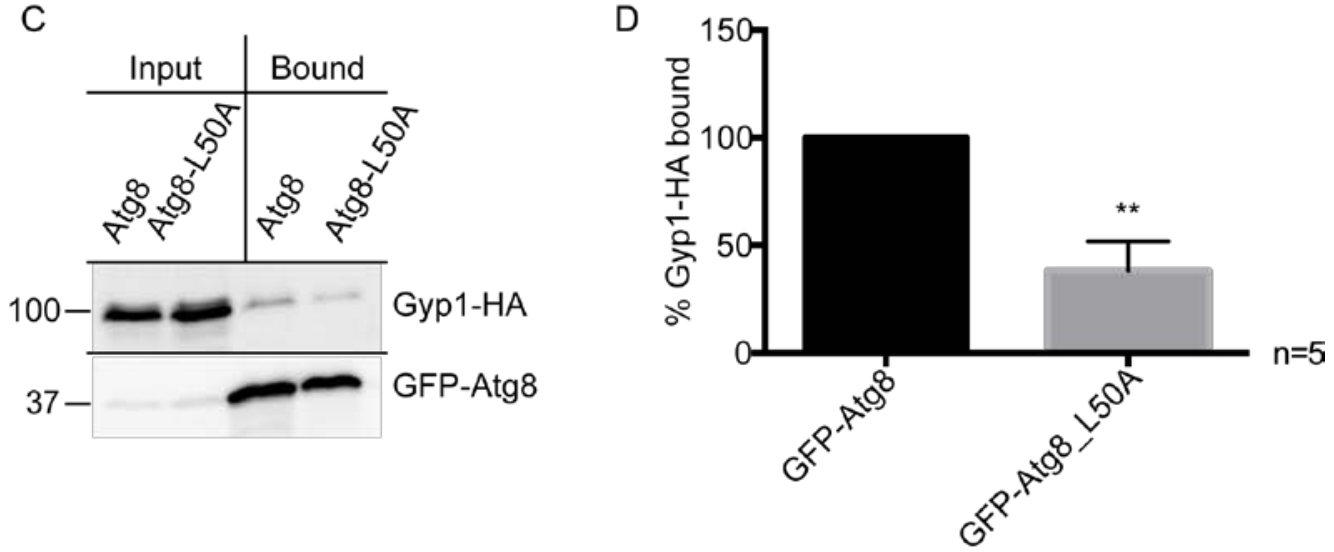

Figure 4.2: Binding of Gyp1 and Atg8 is reduced after mutating the AIM binding sites of Atg8

(A) Analysis of Gyp1-Atg8 interaction using a GFP-TRAP approach. Cells were grown to logarithmic growth phase and then lysed. GYP1 was chromosomally tagged by HA (cGYP1-HA). The GFP-ATG8 constructs were expressed on plasmid from the GAL1 promoter in CGYP1-HA cells. The molecular weight marker is depicted in $\mathrm{kDa}$.

(B) Quantification of six independent experiments revealed that binding of Gyp1HA to GFP-Atg8 was reduced in the GFP-Atg8_L50A and the GFPAtg8_Y49A mutants. These mutations are located in the AIM binding sites of 
Atg8. Binding of Gyp1-HA to GFP-Atg8_FK mutant, located in the NHD of Atg8, was also reduced. Statistics were performed using the one sample ttest. Error bars represent SEM and asterisks represent $p$-values.

(C) Analysis of GFP-Atg8 and Gyp1-HA by a GFP-TRAP approach with endogenous protein amounts. Cells were grown to logarithmic phased and used for the co-precipitation procedure. GYP1 was again chromosomally tagged by HA. GFP-ATG8 was expressed on plasmid from its endogenous promoter. The molecular weight marker is depicted in $\mathrm{kDa}$.

(D) The quantification of five independent experiments revealed that binding of Gyp1-HA to GFP-Atg8_L50A was significantly reduced compared to Atg8 WT. Statistics were performed as indicated in (A).

To test the mechanisms of Gyp1-Atg8 interaction, the known interaction sides in Atg8 were analyzed. Figure 4.2A represents a western blot analysis of the respective GFP-TRAPS. ATG8 mutants were expressed from the GAL1 promoter in the chromosomally tagged GYP1-HA strain. Cells were grown to logarithmic phase before lysis of the cells and co-precipitation of the proteins. After normalization, GFP-Atg8_WT was set to $100 \%$ (Fig.4.2B). Binding of Gyp1-HA to the GFP-Atg8_L50A mutant was significantly reduced $(9.24 \pm 2.88 \%)$. The GFPAtg8_Y49A mutant was reduced to $5.15 \pm 1.03 \%$ and the GFP-Atg8_FK mutant to $42.59 \pm 8.74 \%$. Binding to the GFP-Atg8_ST control was not significantly reduced $(80 \pm 9.57 \%)$. In the presence of the GFP tag alone, binding of Gyp1-HA was not detected (0.55 $\pm 0.18 \%)$ (Fig. 4.2B).

The results presented in Fig 2.4A and B were verified by expressing GFP-Atg8 from its endogenous promoter to confirm the results with approximately native protein quantities. The amount of cells was increased to $1000 \mathrm{OD}_{600}$ and GFPATG8 was expressed from a plasmid under its endogenous promoter. GFPAtg8_L50A was compared to wild type GFP-Atg8. Binding of Gyp1-HA to the GFP-Atg8_L50A mutant was reduced, indicated by analysis of the western blot (Fig. 4.2C). Quantification of five independent experiments revealed that binding was significantly decreased to $38 \pm 13 \%$, compared to wild type set to $100 \%$ after normalization (Fig. 4.2D). These results indicate an interaction of Gyp1 and Atg8 that is mediated by the AIM binding sites of Atg8. 


\subsubsection{The Atg8-interaction motif in Gyp1}

Chapter 4.1.3 revealed that the interaction of Atg8 and Gyp1 occurs via AIM binding sites of Atg8. Mutation of the respective residues Atg8_L50A and Atg8_Y49A led to a reduced interaction of the two proteins, shown by a GFPTRAP approach. As these AIM binding sites of Atg8 usually interact with AIMs, Gyp1 was analyzed for the respective motifs. By using the iLIR software (Kalvari et al., 2014), seven potential AIMs were identified and mutated by substitution of the indicated (in bold) amino acids with alanine (Fig. 4.3A). A sequence alignment (Fig. 4.3B) of Gyp1 shows that the predicted AIMs are conserved among other Saccharomycetes: Kluyveromyces lactis, Naumovozyma castellii, Candida glabrata and Saccharomyces kudriavzevii (Fig. 4.3B). AIM2, AIM5, AIM6 and AIM7 are located in the conserved TBC domain of Gyp1. 
A

B

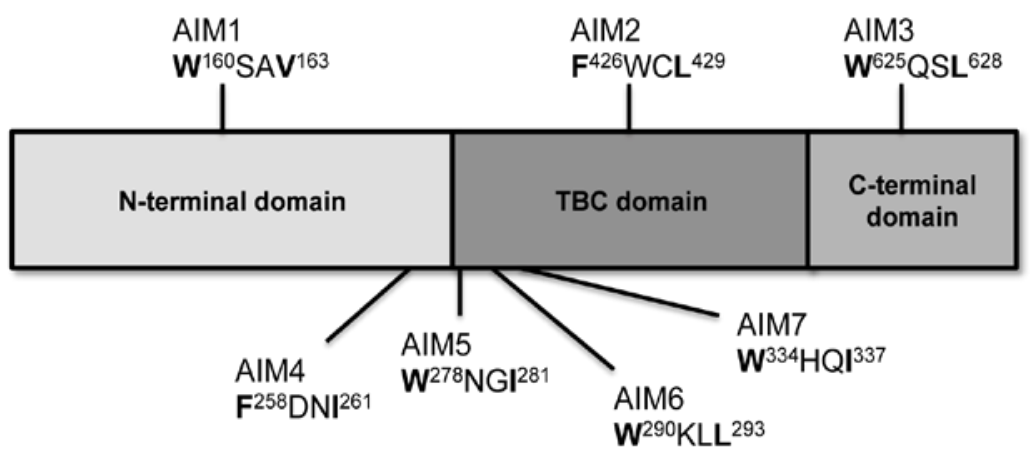

\begin{tabular}{|c|c|c|}
\hline 5. rerevisiae/ $/ 1-637$ & 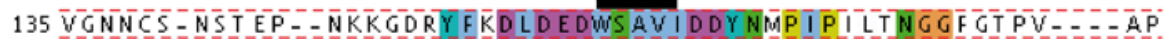 & \\
\hline So 516 & D DKYFK DLDEDWSAVIDDYNMPIPMIN & \\
\hline & GVDSPY - - A - & \\
\hline & AKISRKASE & \\
\hline & $V-\ldots V P$ & 188 \\
\hline-637 & $\bar{N} \bar{D} \bar{S} \overline{K K T} \bar{Q} \bar{L} \bar{E} \bar{I} \bar{N}$ & \\
\hline &.$--I \bar{N} L N R T$ & 170 \\
\hline & $E E-E L D A$ & 243 \\
\hline & QK-ELED & 254 \\
\hline & $\begin{array}{c}\text { DDPKKMQ L I EN } \\
\text { AIM6 }\end{array}$ & 244 \\
\hline 637 & 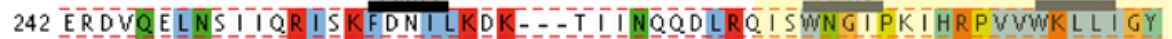 & \\
\hline & $T T Q V N L P E I R K L A W N G I P N$ & 228 \\
\hline & FDDI LKNK- - HI INQQQLRE I SW & 298 \\
\hline & VWWKLL I GY & 309 \\
\hline & WKL L I GY & 2 \\
\hline & QEGFLQRKRKEY & \\
\hline & QEQVLNRKRLEYK & \\
\hline & NPQIPLYQFK & 356 \\
\hline & NPHIPLYQFK & 36 \\
\hline & IDIXOK & 35 \\
\hline & $=2-1$ & \\
\hline & NS LKR I LY FWA & 344 \\
\hline & AS LQKI LYLWA & 414 \\
\hline & TDPLDY & 425 \\
\hline & 58 S VQNSLQR I LYLWA I & 41 \\
\hline 27 & $\mathrm{LT} \overline{\mathrm{KL}}$ & 4 \\
\hline & $\mathrm{LSKL}$ & 402 \\
\hline & Q & 472 \\
\hline & Q & 48 \\
\hline & $\vec{Q}$ & 4) \\
\hline & 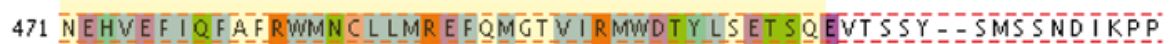 & \\
\hline & $Y$ LSE & 44 \\
\hline & $\gamma \mathrm{L}$ & 530 \\
\hline & Y & 538 \\
\hline & 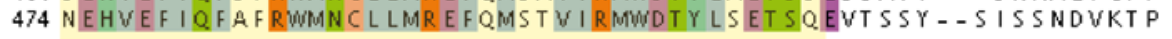 & 529 \\
\hline & $\bar{P} \bar{N} \overline{N A} \bar{E} \bar{E} \bar{S}$ & \\
\hline & RKSRTSSSCP. & 4 \\
\hline & SAT I T & 5 \\
\hline & ASMKSA ..... ESTKLSO & 58 \\
\hline & T EPR VANFATPT KD - FQSPT AALSSMTP - NT VEDSSKMRQS & 58 \\
\hline-6 & D̄ EMLLSEAF IW & 037 \\
\hline & I EMLLSEAY I WQ: & 54 \\
\hline & D I EMLLGEAY I WQS LYKDATS HWL & 642 \\
\hline$C a$ & T KDWDENE I EMLLSEAY I WOS & 642 \\
\hline & & \\
\hline
\end{tabular}

Figure 4.3: Putative AIMs in Gyp1

(A) Potential AIMs of Gyp1 were identified using the iLIR software (Kalvari et al., 2014). Seven potential AIMs were identified and mutated by substitution of the first and the last amino acid of the potential AIM with alanine (indicated in bold).

(B) Sequence alignment of Gyp1 reveals that the predicted AIMs are conserved also in other yeast species. 
The identified potential Gyp1 AIMs were mutated and then analyzed by the GFPTRAP approach, using GFP-Atg8 as bait. GYP1 wild type and the respective AIM mutants were expressed on plasmid from their endogenous promoters. GFPATG8 was expressed from the GAL1 promoter. Western blot analyses indicated a reduced binding of Gyp1_AIM1-HA to GFP-Atg8 (Fig. 4.4A). Quantification of eight independent experiments revealed a significant reduction in the interaction of Gyp1_AIM1-HA and GFP-Atg8 to $3.77 \pm 1.03 \%$ compared to Gyp1_WT-HA (set to $100 \%$ after normalization) (Fig. 4.4B). Binding of the Gyp1_AIM3-HA mutant protein to GFP-Atg8 is increased to $177.73 \pm 29.21 \%$. With exception of AIM1 and AIM3, all other AIM mutations in Gyp1 seemed to destabilize the protein as the levels were strongly reduced compared to the Gyp1 wild type protein (Fig. 4.4C). However, Gyp1_AIM2-HA was included in the GFP-TRAP analysis of Gyp1 and Atg8. Binding of Gyp1_AIM2-HA (64.82 $\pm 23.24 \%$ ) to GFPAtg8 was not significantly affected, although a clear interpretation of the data was difficult, due to instability of the protein.

In further studies, the binding of Atg8 and Gyp1 was analyzed using the Gyp1_R343K mutant, which has been reported to have no GAP activity (Albert et al., 1999). The GYP1_WT and the GYP1_R343K mutant were both expressed from their endogenous promoters and tagged by HA. GFP-ATG8 was expressed from the GAL1 promoter (Fig. 4.4D). Binding of Gyp1-HA and Gyp1_R343K-HA to GFP-Atg8 wild type was compared. After quantification and normalization of the western blots, interaction of Gyp1_R343K-HA and GFP-Atg8 showed a binding of $94.35 \pm 9.79 \%$, which was not significantly different from Gyp1_WT-HA, set to $100 \%$ (Fig. 4.4E). Gyp1_WT-HA interacts to $0.40 \pm 0.33 \%$ with the GFP-tag, used as negative control. These results indicate an AIM-dependent interaction that is independent from the GAP activity of Gyp1. 


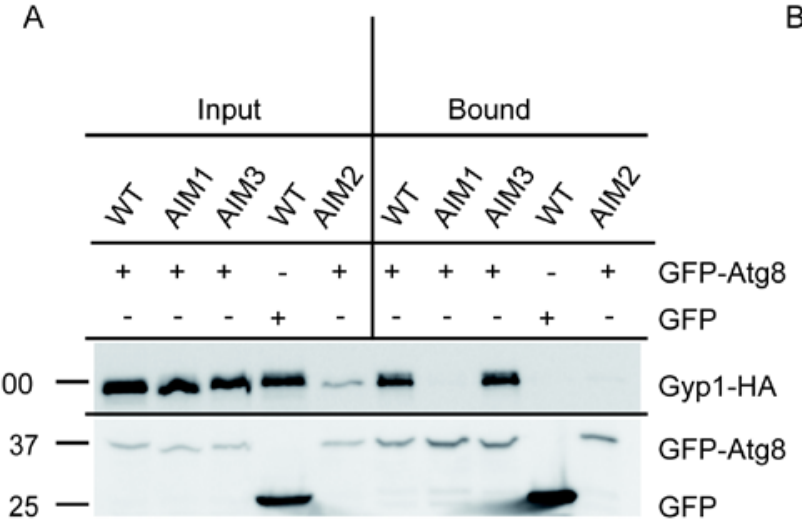

C
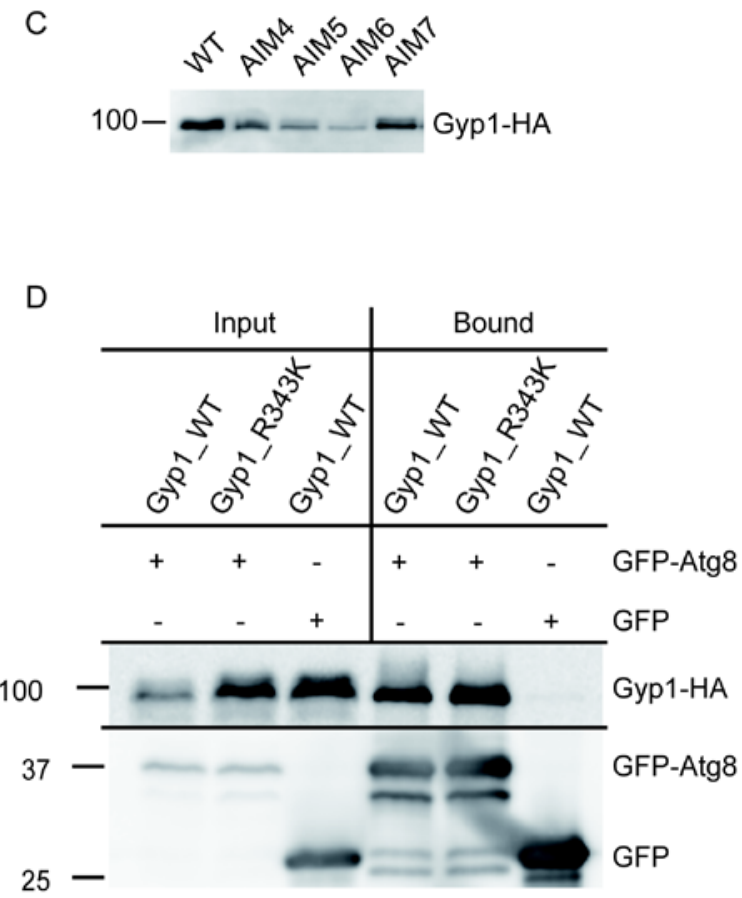

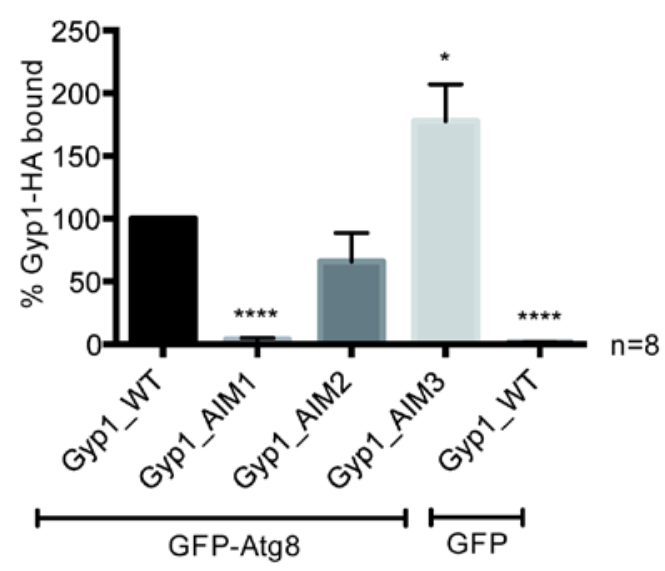

E

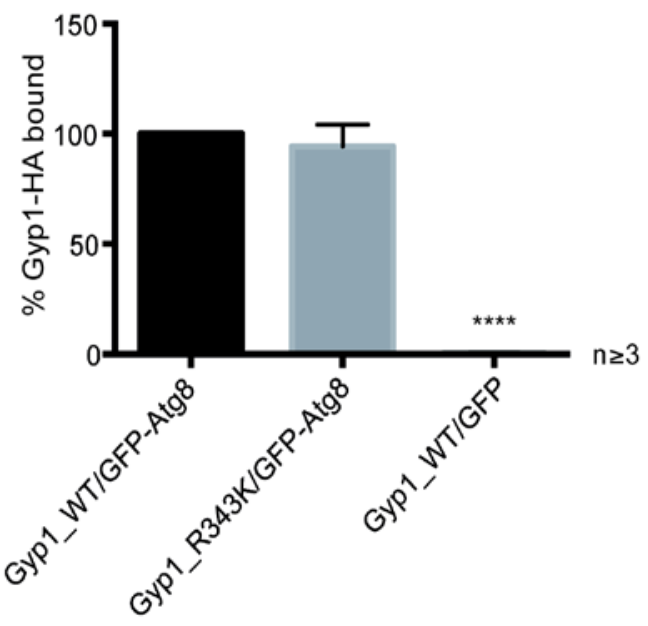

Figure 4.4: Binding of Gyp1_AIM1 to Atg8 is reduced in vivo

(A) Binding of Gyp1-HA and GFP-Atg8 was analyzed using a GFP-TRAP approach. Cells were grown to logarithmic phase and then lysed. GFP-ATG8 was expressed from the GAL1 promoter on plasmid (pYES2). GYP1 and the respective mutants were tagged by $\mathrm{HA}$ and expressed from their endogenous promoters in gyp1s atg8s cells. Mutation of Gyp1_AIM1-HA led to a decreased binding to GFP-Atg8. Mutation of AIM2 in Gyp1 seemed to destabilize the protein because less protein was detected by western blot analysis. Binding of Gyp1_AIM3-HA to GFP-Atg8 was increased. The molecular weight marker is depicted in $\mathrm{kDa}$.

(B) Analyses of eight experiments revealed that the reduced binding of Gyp1_AIM1-HA and GFP-Atg8 is highly significant. Mutation of AIM3 seemed to increase the interaction with GFP-Atg8. Gyp1_AIM2-HA did not significantly differ in binding to GFP-Atg8 compared to the Gyp1_WT-HA protein. Statistics were done using the one sample t-test. Error bars represent SEM and asterisks represent $p$-values. 
(C) Western blot analysis of Gyp1 AIM mutants. Cells were grown to logarithmic growth phase and lysed, according to the GFP-TRAP protocol. Mutation of AIM4 to AIM7 caused instability of the Gyp1 protein shown by lower protein levels. The experiments were performed as indicated in $(A)$. The molecular weight marker is depicted in $\mathrm{kDa}$.

(D) Western blot analysis of Gyp1_R343K-HA. The Gyp1_R343K-HA mutant, which lacks the GAP activity, interacted with GFP-Atg $\overline{8}$ in a wild type-like manner. These experiments were performed using the same conditions as in (A). The molecular weight marker is depicted in $\mathrm{kDa}$.

(E) The quantification of the western blot experiments revealed that binding of Gyp1_WT-HA and Gyp1_R343K-HA to GFP-Atg8 was not significantly different. Gyp1-HA did not bind to GFP tag alone. Statistics were performed as indicated in (B).

\subsection{Analysis of Gyp1 in selective autophagy}

Selective autophagy mediates the selective degradation of aggregated proteins, damaged organelles and invading pathogens. Aminopeptidase I (Ape1) is constitutively transported to the vacuole via the Cvt-pathway (Chapter 2.4.1). To investigate the role of Gyp1 and its predicted interaction with Atg8 in selective forms of autophagy, the Cvt-pathway and mitophagy were further analyzed.

\subsubsection{Gyp1 is needed for proper proceeding of the Cvt-pathway}

Proceeding of the Cvt-pathway was measured by maturation of pApe1. The premature form of Ape1 (pApe1) is transported to the vacuole where it is matured into mApe1. In the vacuole, mApe1 fulfills its function in the degradation of proteins. The processing of pApe1 can be monitored by a molecular weight shift of the protein in immunoblot analysis. Deletion of ATG1 completely blocks the Cvt-pathway and no mApe1 is detected. Thus, the $\operatorname{atg} 1 \Delta$ strain was used as a negative control. The GAPs Gyp5 and Gyp8 were included into this analysis. GYP1, GYP5 and GYP8 were deleted and analyzed by western blot as well as the knockout combinations of these proteins (Fig. 4.5A, C, E). Ape1 was detected on the western blot by an Ape1 antibody. The Ape1 maturation rate was determined by calculating the ratio of mApe1 from the total amount of Ape1. 
A

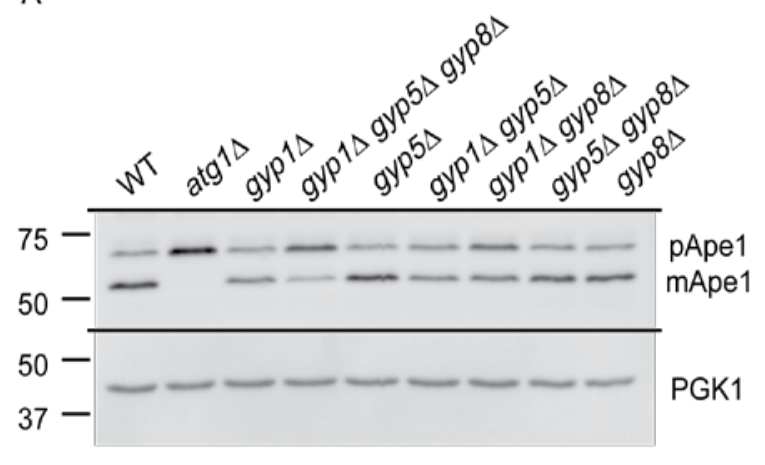

C
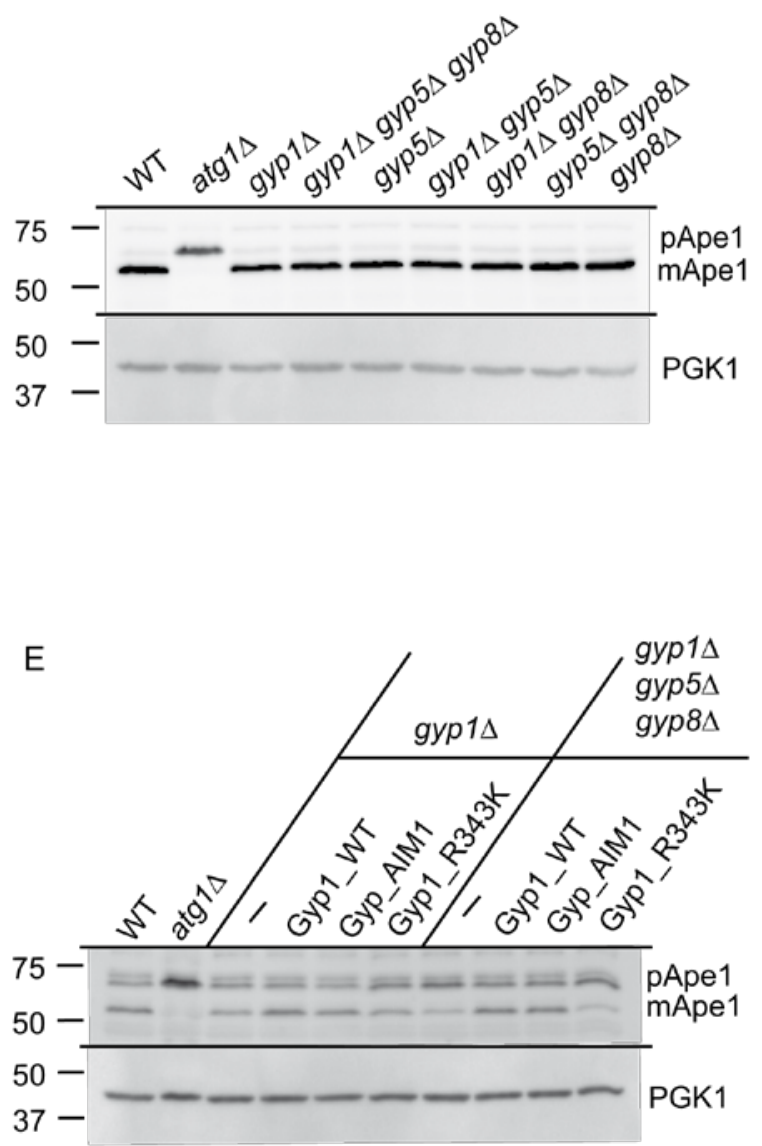

B

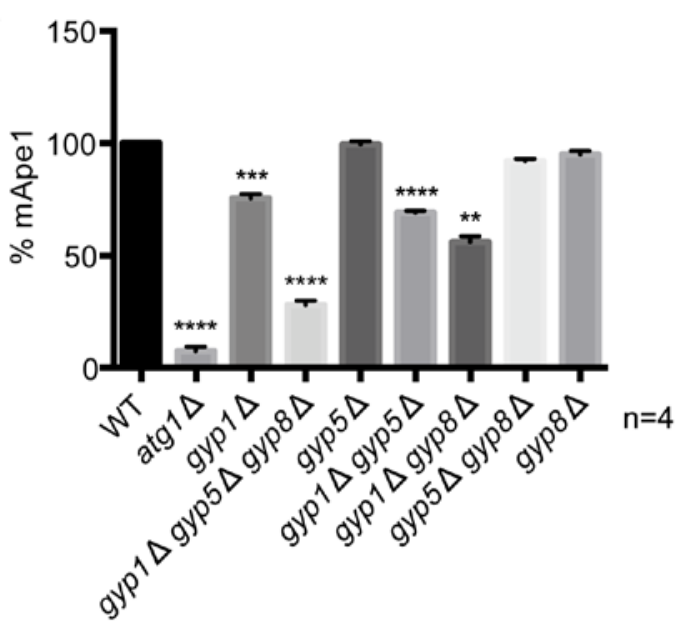

D
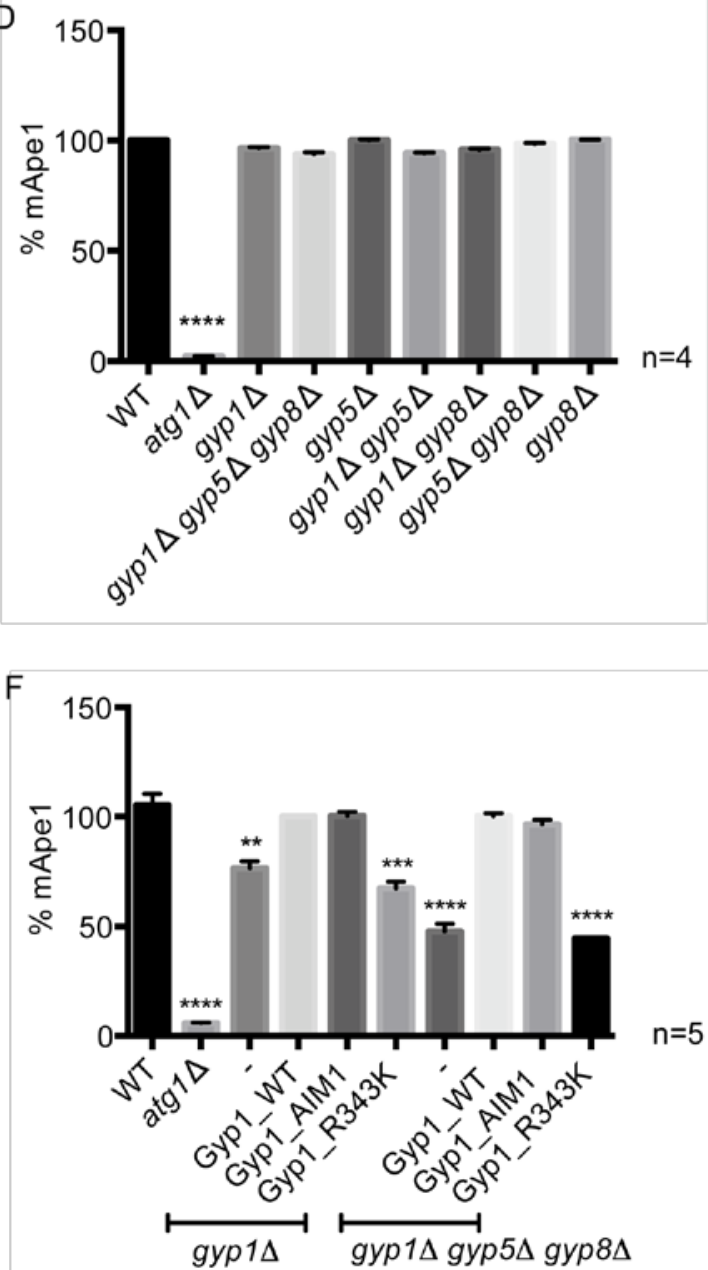

Figure 4.5: pApe1 processing is impaired upon GYP1 deletion under nutrient-rich conditions

(A) Analysis of pApe1 maturation under nutrient-rich conditions. The maturation rate of pApe 1 is reduced in gyp $1 \Delta$ cells and in gyp $1 \Delta$ gyp $5 \Delta$ gyp $8 \Delta$ cells. Cells were grown to logarithmic phase. Ape1 was detected with an Ape1 antibody and PGK1 (3-phosphoglycerate kinase 1) with a PGK1 antibody. WT and $\operatorname{atg} 1 \Delta$ cells were used as control. The molecular weight marker is depicted in $\mathrm{kDa}$. 
(B) Quantification of four independent pApe1 maturation experiments. Single deletion of GYP5 and GYP8 caused no reduction, but upon additional deletion of GYP1, the pApe1 maturation rate declined. The most severe phenotype was observed in gyp1 $1 \Delta y p 5 \Delta$ gyp $8 \Delta$ cells. The pApe1 maturation rate was determined by calculating the ratio of mApe1 from the total amount of Ape1. Gyp1_WT was set to $100 \%$. Statistics were done using the one sample t-test. Error bars represent SEM and asterisks represent $p$-values.

(C) Analysis of pApe1 maturation by western blot after induction of autophagy. Induction of autophagy resulted in wild type levels of pApe1 maturation in all strains under investigation. Samples were taken after $4 \mathrm{~h}$ incubation of the cells in nitrogen-lacking medium (SD-N).

(D) The western blot analysis (C) of four independent experiments was quantified. Statistical analysis with the unpaired one sample t-test revealed no significant differences between the GYP deletion strains. Statistics were performed as indicated in (B).

(E) The pApe1 maturation rate of the Gyp1 mutant proteins Gyp1_AIM1 and

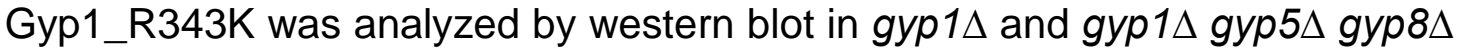
cells under logarithmic growth conditions.

(F) Maturation of pApe1 proceeds at wild type level after mutation of AIM1 in Gyp1, but is reduced in the GAP activity mutant Gyp1_R343K.

The pApe1 maturation rate of the wild type strain was set to $100 \%$ after normalization. The GYP1 deletion resulted in a reduced pApe1 maturation rate of $75.62 \pm 1.82 \%$ (Fig. 4.5B). Deletion of GYP5 and GYP8 caused no significant

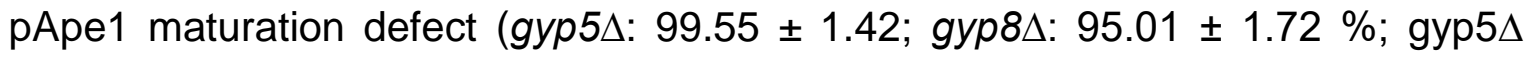
gyp8 $\Delta: 92.11 \pm 0.99 \%)$. Additional deletion of GYP1 slightly increased the pApe1

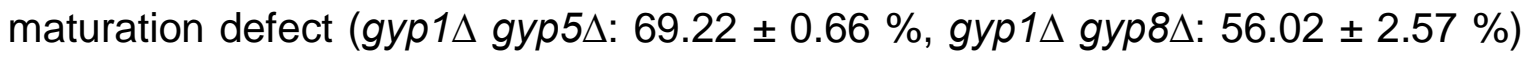
and deletion of all three GAPs caused a reduction in pApe1 maturation to $28.05 \pm$ $1.88 \%$ (Fig. 4.5B). These results indicated that Gyp1 is important for proper recruitment of pApe1 to the vacuole. Furthermore, Gyp1 might be the major GAP for this pathway, as a deletion of GYP5 or GYP8 as well as the double deletion of both genes had no significant phenotype. The severe pApe1 maturation defect in

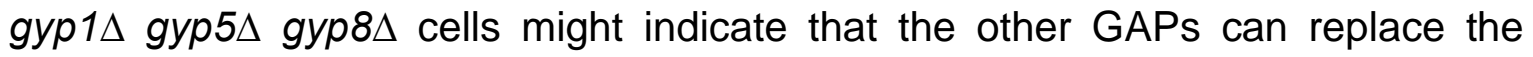
function of Gyp1 at least partially.

Under starvation conditions, the transport of pApe1 is taken over by unselective macroautophagy and the Cvt-complex is engulfed by the autophagosome (Baba et al., 1997). To induce starvation, cells were shifted to nitrogen-lacking medium and incubated for $4 \mathrm{~h}$. The observed phenotype under nutrient-rich conditions was absent under these conditions (Fig. 4.5C). Only the pApe1 maturation rate of the $\operatorname{atg} 1 \Delta$ negative control was significantly reduced to $2.26 \pm 0.08 \%$ compared to the 
WT set to $100 \%$ (Fig. 4.5D). The pApe1 maturation rate of the gyp1 $1 \Delta$ strain was reduced to $96.51 \pm 0.05 \%$, gyp $1 \Delta$ gyp $5 \Delta$ gyp $8 \Delta$ to $93.83 \pm 0.83 \%$, gyp $1 \Delta$ gyp5 to $94.25 \pm 0.25 \%$, gyp $1 \Delta$ gyp $8 \Delta$ to $95.87 \pm 0.42 \%$ and gyp5 gyp $8 \Delta$ to $98.45 \pm$ $0.43 \%$. Analysis of gyp $5 \Delta$ cells and gyp $8 \Delta$ cells led to values of $100.14 \pm 0.37 \%$ and $100.38 \pm 0.09 \%$ compared to WT (Fig. 4.5D). These differences in pApe1 maturation were not significant compared to the wild type. This led to the suggestion that the Gyp proteins and especially Gyp1 might be involved in selective forms of autophagy under nutrient-rich conditions but not in unselective autophagy.

To further analyze the molecular basis of the observed phenotype, a complementation analysis was performed. GYP1_WT, GYP1_AIM1 and GYP1_R343K were expressed untagged on a plasmid from their endogenous

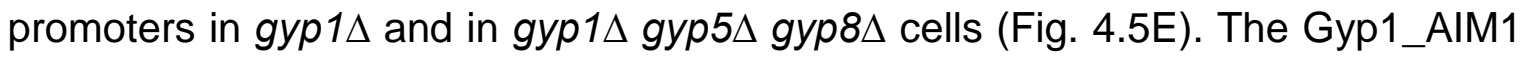
mutant is impaired in its interaction with Atg8 and the Gyp1_R343K mutant lacks

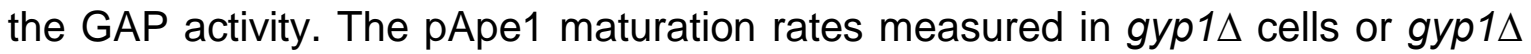
gyp5 $5 y$ gy 8 cells, expressing the GYP1_WT plasmid, were set to $100 \%$ (Fig. 4.5F). To examine the functionality of the plasmid-expressed GYP1_WT, pApe1 maturation was measured in a wild type strain expressing an empty plasmid. This wild type strain (with an pApe1 maturation of $105.5 \pm 5.18 \%$ ) as well as the

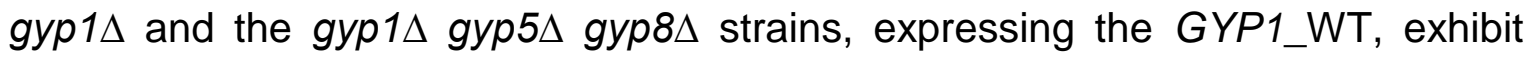
similar pApe1 maturation rates, indicating a functional plasmid-expressed GYP1_WT.

The Gyp1_R434K mutant had a reduced pApe1 maturation rate of $67.37 \pm 3.05 \%$ in gyp $1 \Delta$ cells and a reduction to $44.57 \pm 0.51 \%$ in gyp $1 \Delta$ gyp5 $5 y p 8 \Delta$ cells (Fig. 4.5F). GYP1_AIM1 in both strains revealed no significant reduction in pApe1

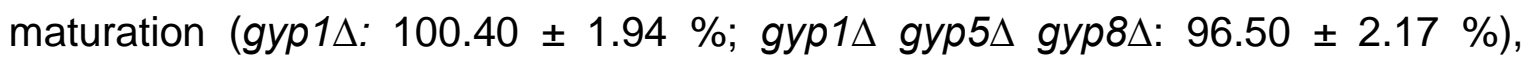
indicating that the GAP activity of Gyp1 is responsible for the pApe1 maturation phenotype and not the defect in binding of Gyp1 to Atg8. The $\operatorname{atg} 1 \Delta$ strain was used as a negative control where pApe1 maturation is blocked. As expected the pApe1 maturation rate was reduced to $5.75 \pm 0.30 \%$ in this strain (Fig. 4.5F). 


\subsubsection{Gyp1 is important for proper proceeding of post-log Mitophagy}

Mitophagy was measured by using the mito-GFP Su9-DHFR plasmid. Mitophagy leads to the degradation of the non-GFP part of the marker protein. The GFP-tag is cleaved off in the vacuole as mitophagy proceeds and the amount of free GFP can be analyzed on western blot by detection with a GFP antibody. The ATG32 deletion strain was used as a negative control. Atg32 functions as a receptor in mitophagy and is upregulated under respiratory growth conditions. Upon deletion of ATG32, mitophagy is blocked (Kanki et al., 2009).

On plasmids, GYP1, GYP1_AIM1 and GYP1_R343K were expressed untagged from the GYP1 promoter; the plasmid-encoded mito-GFP was expressed from the MET25 promoter. Samples were taken after $0 \mathrm{~h}, 48 \mathrm{~h}$, and $72 \mathrm{~h}$ of incubation in lactate medium without methionine to induce expression of the mito-GFP marker (Fig. 4.6A). For calculation of the mitophagic rate, the amount of free GFP was divided be the amount of mito-GFP. Therefore the $48 \mathrm{~h}$ samples were used. The sample expressing the wild type GYP1 in gyp1 $1 \Delta$ cells was set to $100 \%$ (Fig. 4.6B). The Gyp1_WT protein led to wild type levels of mitophagy, compared to the wild type strain expressing an empty plasmid. This indicated a functional plasmidencoded GYP1. The ATG32 deletion strain displayed the expected reduction in the mitophagic rate to $2.5 \pm 0.5 \%$. Deletion of GYP1 (gyp1 $1 \Delta$ cells expressing an empty plasmid), reduced the mitophagic rate to $51.0 \pm 4.1 \%$. The Gyp1_AIM1 mutant resulted in a reduction to $80.75 \pm 4.1 \%$ and the Gyp1_R343K mutant caused a reduction to $62.0 \pm 3.8 \%$ (Fig. 4.6B). These results indicate that the Gyp1 GAP activity as well as the binding of Gyp1 to Atg8 might contribute to proper proceeding of mitophagy. 


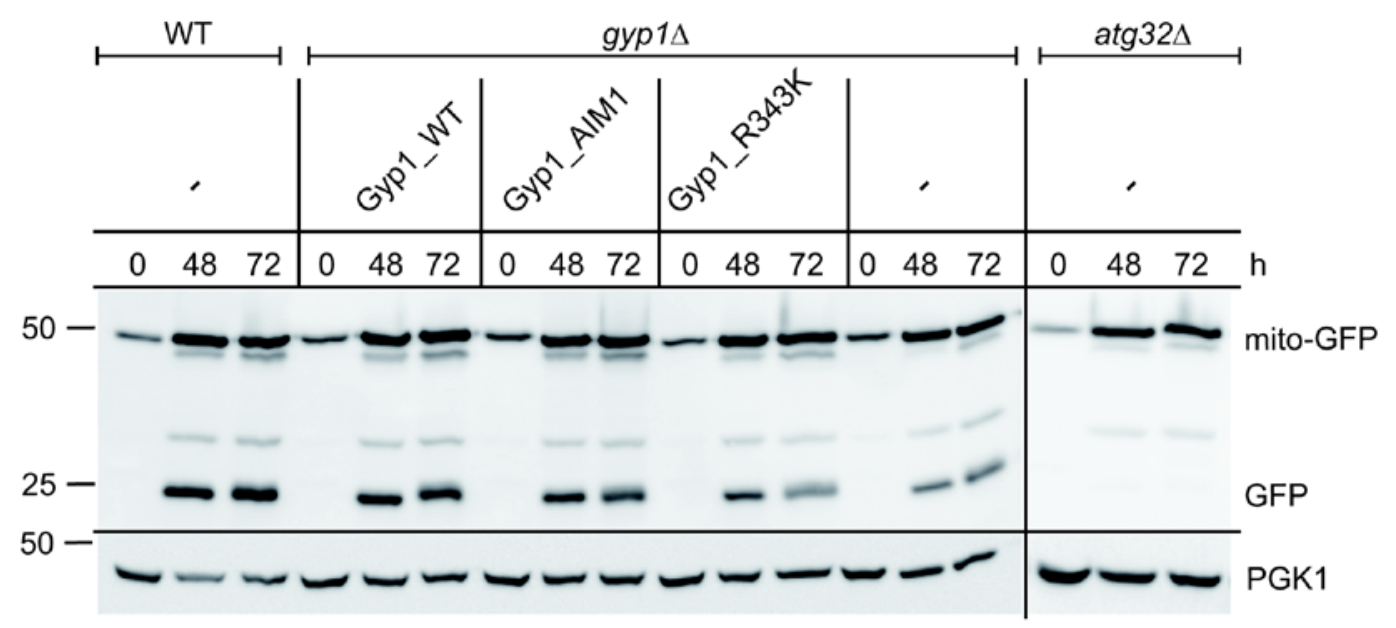

B

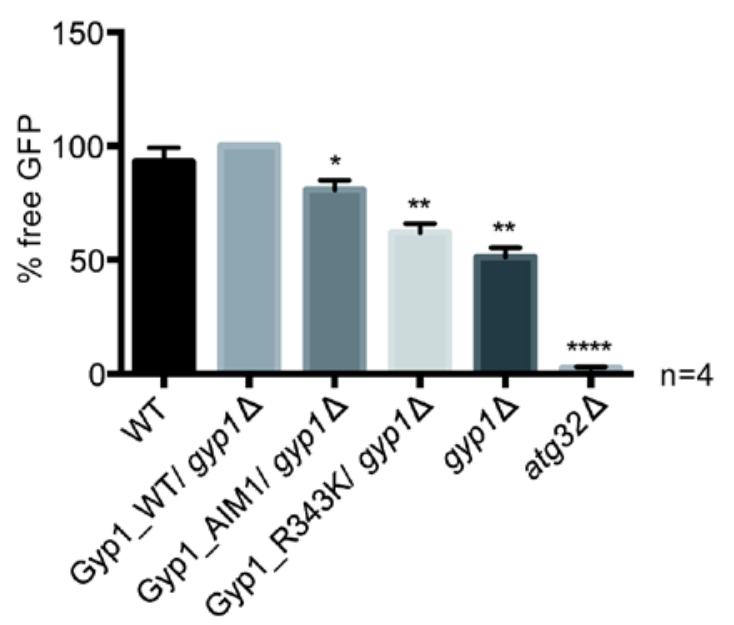

Figure 4.6: Gyp1 plays a role in proceeding of post-log mitophagy

(A) The mitophagic rate was monitored by expression of the mito-GFP plasmid together with the respective GYP1 plasmids under investigation or empty plasmids (-). Upon degradation of the mito-GFP protein in the vacuole, free GFP is released. Therefore, free GFP indicates the proceeding of mitophagy and was detected by western blot. Cells were grown in lactate medium for $72 \mathrm{~h}$. Samples were taken after $0 \mathrm{~h}, 48 \mathrm{~h}$ and $72 \mathrm{~h}$. A GFP antibody detected the mito-GFP protein and the free GFP. A PGK1 antibody detected PGK1 as a loading control. The molecular weight marker is depicted in $\mathrm{kDa}$.

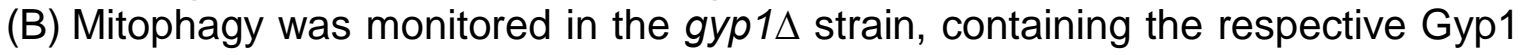
proteins under investigation. Upon deletion of GYP1, the mitophagic rate was reduced and could not be completely restored by the mutant proteins Gyp1_AIM1 and Gyp1_R343K. The mitophagic rate of Gyp1_WT was set to $100 \%$ and the statistical analysis was performed using the one sample t-test. Error bars represent SEM and asterisks represent $p$-values. For the calculation of the mitophagic rate, the $48 \mathrm{~h}$ values were used. Free GFP was divided by the amount of mito-GFP. 


\subsection{Gyp1 and Atg8 co-localize using fluorescence microscopy}

To support the detected interaction of Gyp1 and Atg8, the proteins were analyzed under growing conditions by using the Delta Vision microscope (Fig. 4.7A). GFPATG8 was expressed from its endogenous promoter; Cherry-GYP1 was expressed from the MET25 promoter $(0.3 \mathrm{mM}$ methionine was added to the medium) in the atg $8 \Delta$ gyp1 $\Delta$ strain. GFP-Atg8 co-localized to $28.62 \pm 2.63 \%$ with one of multiple Cherry-Gyp1 dots (Fig. 4.7B). Gyp1 is reported to localize to the Golgi (Du and Novick, 2001), indicating that the dots that do not co-localize with GFP-Atg8 represent the Golgi. The Cherry-Gyp1_AIM1 mutant (26.10 $\pm 4.11 \%)$ and the Cherry-Gyp1_R343K mutant (22.14 $\pm 6.70 \%)$ showed a wild type-like colocalization rate with GFP-Atg8 (Fig. 4.7B). Functionality of the Cherry-Gyp1 plasmid was analyzed by western blot (Fig. 4.7C). Compared to the wild type strain set to $100 \%$, the gyp1D strain expressing the Cherry-GYP1 plasmid showed no significant reduction in pApe1 maturation (95.75 $\pm 2.72 \%)$. The pApe1 maturation rate in the gyp1 1 strain was reduced to $48.75 \pm 2.01 \%$ (Fig. 4.7D). This indicates functionality of Cherry-Gyp1. Atg8 can be used as a PAS marker (Suzuki et al., 2010; Kirisako et al., 1999). Thus, the interaction of Gyp1 and Atg8 likely occurs at the PAS. 


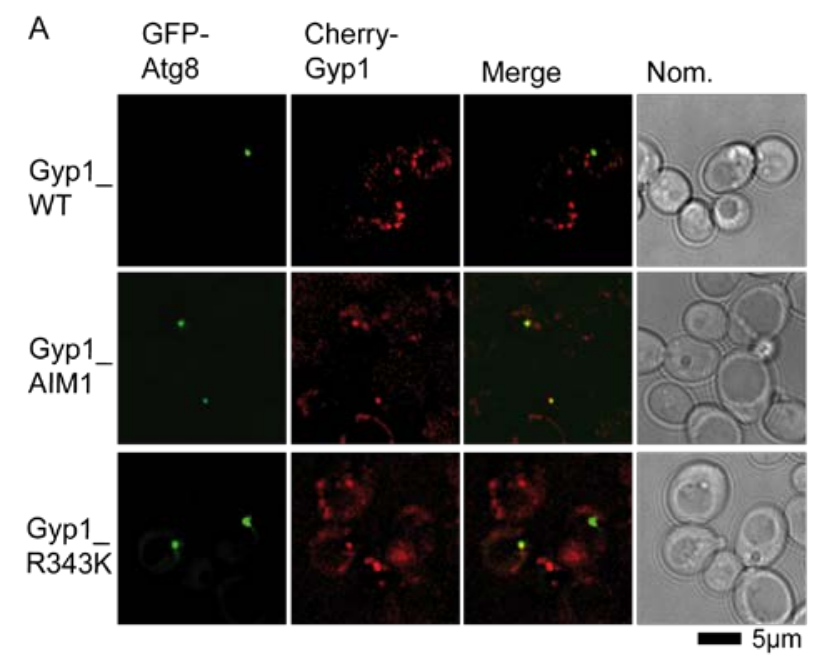

B
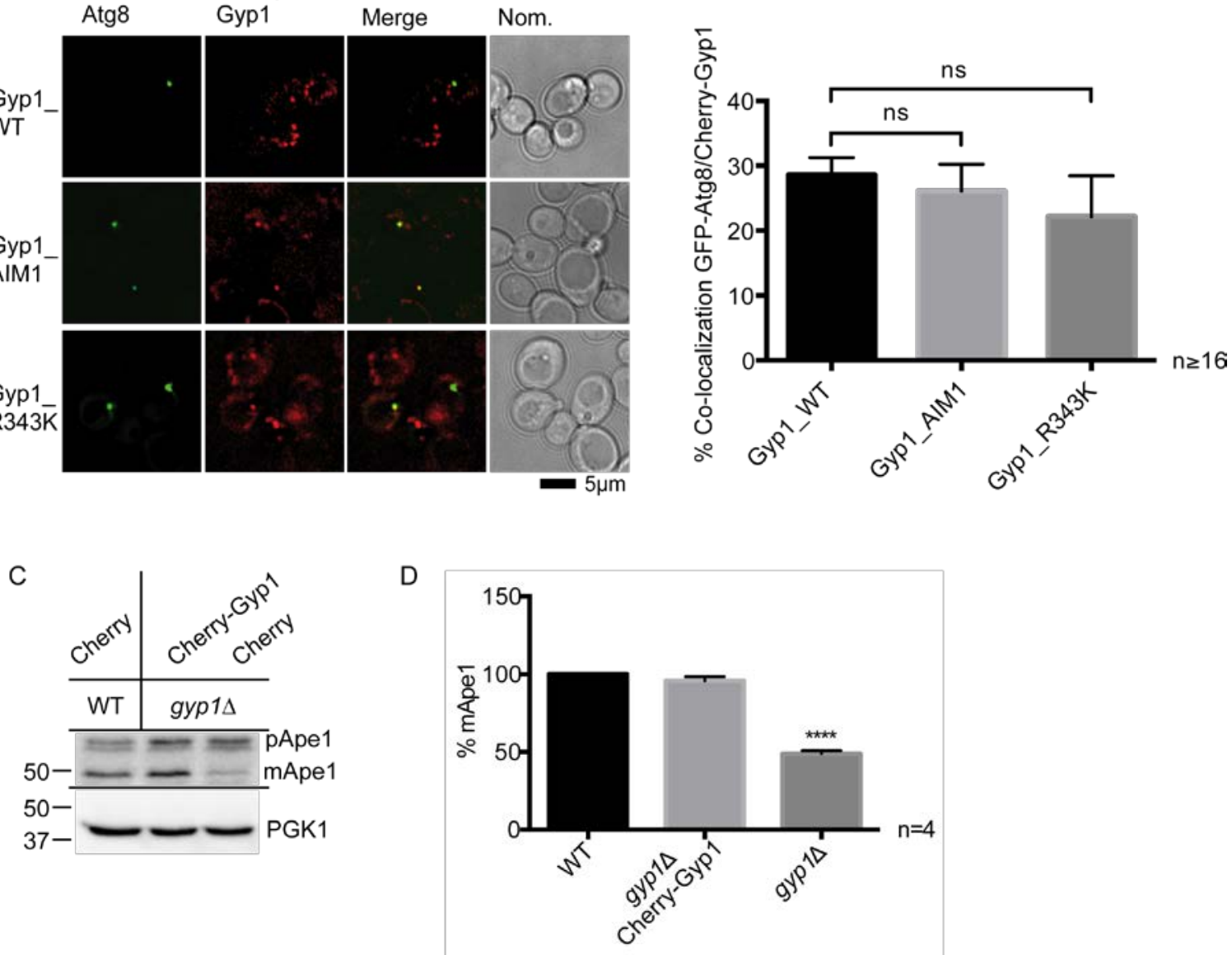

Figure 4.7: GFP-Atg8 and Gyp1 co-localize

(A) Cherry-Gyp1 and GFP-Atg8 dots were analyzed for co-localization, using the Delta Vision microscope. The cells were grown to logarithmic phase. For the experiment the atg8 $8 y$ gy $1 \Delta$ strain was used. Cherry-GYP1 was expressed from the MET25 promoter and GFP-ATG8 was expressed from its endogenous promoter.

(B) The percentage of Cherry-Gyp1 dots that co-localize with GFP-Atg8 was determined. A subfraction of multiple Cherry-Gyp1 dots co-localized with GFP-Atg8. There were no significant differences detectable in the colocalization rate of GFP-Atg8 and Cherry-Gyp1_AIM1 or CherryGyp1_R343K. Cherry-Gyp1_WT dots/cell were set to $100 \%$. Statistics were performed using the unpaired two tailed t-test. $\mathrm{n}$ indicates the number of pictures used for the analysis. Pictures were taken in at least three independent experiments. Error bars represent SEM.

(C) The Cherry-Gyp1 protein was tested for its functionality by measurement of the pApe1 maturation rate. Cells were grown to logarithmic phase. The Cherry plasmid was experessed in WT and gyp1s cells and compared to the CherryGYP1 plasmid, expressed in gyp1s cells. The molecular weight marker is depicted in kDa.

(D) Quantification of four experiments resulted in similar amounts of mApe1 compared to wild type, indicating functionality of Cherry-Gyp1. The pApe1 maturation rate of the wild type strain was set to $100 \%$. Statistics were calculated using the one sample t-test. Error bars represent SEM and asterisks represent $p$-values. 


\subsection{Analysis of the Cvt-pathway using fluorescence microscopy}

Results of the GFP-TRAP approach indicated that Gyp1 and Atg8 interact (Chapter 4.3). Furthermore, this interaction is likely to occur at the PAS, as demonstrated by fluorescence microscopy (Chapter 4.3). This interaction might be important during selective forms of autophagy as a phenotype was observed for the Cvt-pathway under growing conditions and for mitophagy (Chapter 4.2.1 and 4.2.2). To further analyze the role of Gyp1 during selective autophagy, different components of the autophagic machinery were analyzed.

\subsubsection{Localization of pApe1 and Atg11 at the PAS does not require Gyp1}

To determine at which step Gyp1 is needed, the assembly of the Cvt-complex was further analyzed in atg $1 \Delta$, gyp $1 \Delta$ and gyp $1 \Delta$ gyp5 gyp8s strains. Proper assembly of the Cvt-complex was monitored by formation of Ape1-RFP dots in the cells. APE1-RFP was expressed from a single copy plasmid under its endogenous promoter and analyzed by fluorescence microscopy (Fig. 4.8A). Compared to the wild type strain (0.28 \pm 0.02 dots/cell), the number of Ape1-RFP dots per cell did not change significantly in the gyp1 $0.25 \pm 0.02$ dots/cell) and the gyp $1 \Delta$ gyp $5 \Delta$ gyp8 8 (0.32 \pm 0.02 dots/cell) strain. The atg1 $\Delta$ strain showed an increased number of Ape1-RFP dots per cell $(0.36 \pm 0.02)$, which can be explained by a block in pApe1 transport into the vacuole upon ATG1 deletion (Fig. 4.8B). 
A

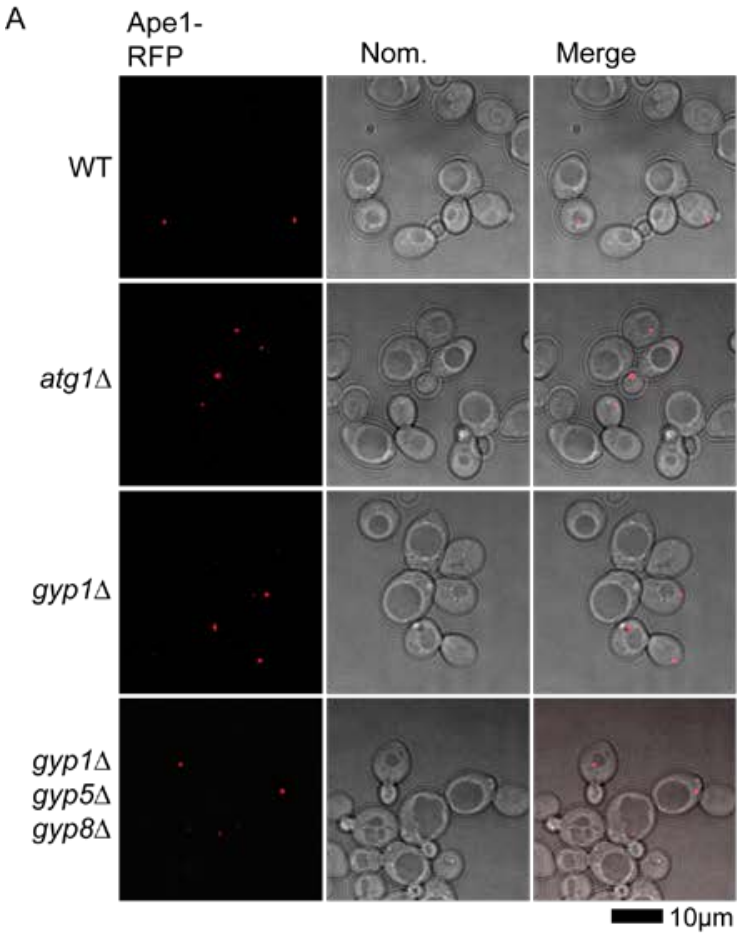

C

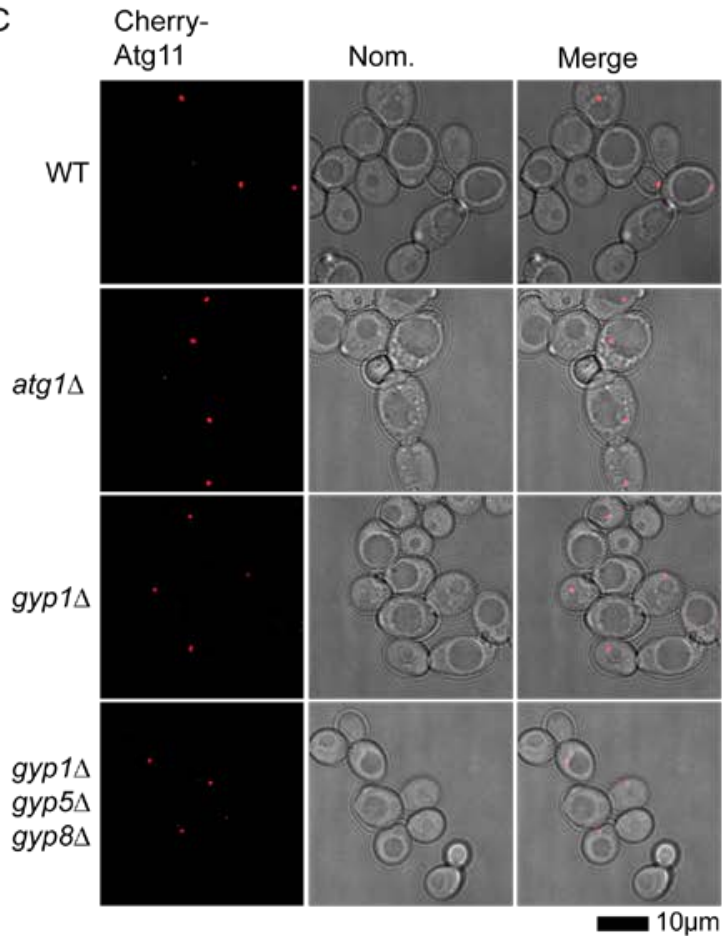

B

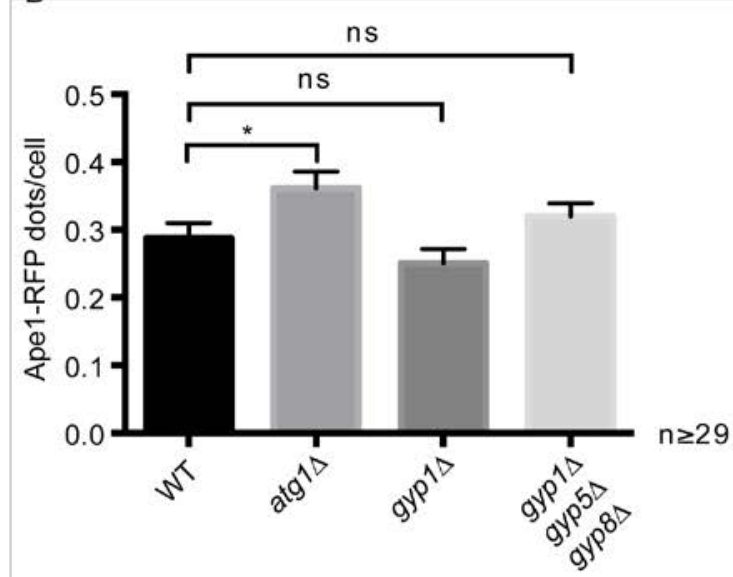

D

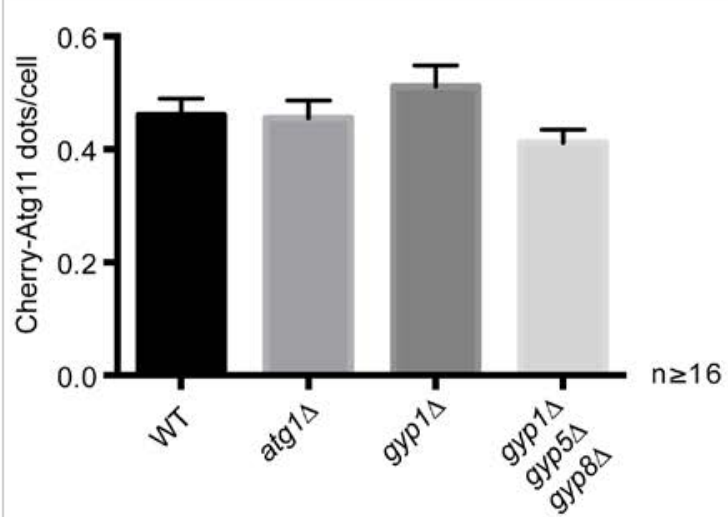

Figure 4.8: Analysis of the localization of Ape1-RFP and Cherry-Atg11 upon deletion of the GYP genes

(A) Ape1-RFP was visualized in the indicated strains by the Delta Vision microscope. Cells were grown to logarithmic phase. APE1-RFP was expressed from a plasmid under its endogenous promoter.

(B) The number of Ape1-RFP dots/cell did not differ significantly in the gyp1 $\Delta$ and the gyp1 gyp5 $\Delta$ gyp8 $\Delta$ strain compared to the WT strain. Statistics were performed using the unpaired two tailed t-test. $\mathrm{n}$ indicates the number of pictures used for the analysis. Pictures were taken in at least three 
independent experiments. Error bars represent SEM and asterisks represent p-values.

(C) Cherry-Atg11 dots/cell were also analyzed under logarithmic growth conditions using the Delta Vision microscope. Cherry-ATG11 was expressed from the MET25 promoter and $0.3 \mathrm{mM}$ methionine was added to the medium.

(D) The number Cherry-Atg11 dots/cell was not significantly different in WT cells, gyp $1 \Delta$ and gyp $1 \Delta$ gyp5 gyp8s cells, indicating proper assembly of the Cvtcomplex and assembly with Atg11. $\mathrm{n}$ indicates the number of pictures taken for the analysis. Pictures were taken in at least three independent experiments. Statistics were performed as indicated in (B).

During Cvt-vesicle formation, the specific scaffold protein Atg11 is recruited and assembles with the Cvt-complex (Yormitsu and Klionsky, 2005). Atg11 is important for biogenesis of the Cvt-vesicles and has been reported as an effector of Ypt1 (Lipatova et al., 2012). Cherry-ATG11 was expressed from the MET25 promoter (0.3 $\mathrm{mM}$ methionine was added to the medium). Cells were grown to logarithmic growth phase and then analyzed using the Delta Vision microscope (Fig. 4.8C). The number of Cherry-Atg11 dots per cell revealed no significant changes comparing the wild type cells with $0.46 \pm 0.03$ dots/cell to atg $1 \Delta$ cells (0.44 \pm 0.03 dots/cell), gyp $1 \Delta$ cells (0.51 \pm 0.04 dots/cell) and gyp $1 \Delta$ gyp $5 \Delta$ gyp $8 \Delta$ cells (0.41 \pm 0.02 dots/cell) (Fig. 4.8D).

Thus, an improper recruitment or localization of Atg1 and Atg11 was not detectable after deletion of GYP1 or of GYP1, GYP5 and GYP8. This indicates proper assembly of the Cvt-complex and assembly with Atg11.

\subsubsection{Gyp1 plays a role in disassembly of the Ypt1-Atg1 complex}

Atg1 has been previously discovered as an interaction partner of Ypt1 (Wang et al., 2013), which is negatively regulated by Gyp1 (Du and Novick, 2001). To monitor Atg1, the gene was tagged by GFP and expressed under control of its endogenous promoter from a plasmid. Distribution of the Atg1-GFP protein was examined by fluorescence microscopy (Fig. 4.9A). The atg1s strain (expressing ATG1-GFP from plasmid) was used as wild type sample and analyzed together with atg1 $1 \Delta y p 1 \Delta$ and atg $1 \Delta$ gyp $1 \Delta$ gyp5 $\Delta$ gyp8 $\Delta$ cells. Compared to the wild type control with $0.32 \pm 0.02$ dots/cell, both, atg1 $\operatorname{gyp} 1 \Delta$ (0.43 \pm 0.02 dots/cell) and $\operatorname{atg} 1 \Delta$ gyp $1 \Delta$ gyp5 gyp8 cells (0.42 \pm 0.02 dots/cell) showed an increase in 
Atg1-GFP dots/cell (Fig. 4.9B). This indicates that the Atg1 function might be impaired by deletion of GYP1 and thereby progress in PAS assembly.

To further examine the observed effect of Atg1-GFP accumulation at the PAS upon deletion of GYP1, GFP-TRAPs were performed. Here, the complex of Ypt1 and Atg1 was analyzed. ATG1-GFP was expressed from a plasmid in atg1 1 and $\operatorname{atg} 1 \Delta$ gyp1s. Proteins were analyzed under logarithmic growth conditions (Fig. 4.9C). A Ypt1 antibody was used to detect endogenous Ypt1 that co-precipitated with Atg1-GFP. The amount of co-precipitated Ypt1 increased upon GYP1 deletion (Fig. 4.9C). Compared to the WT (normalized and set to $100 \%$ ) the amount of co-precipitated of Ypt1 increased to $172.1 \pm 27.77 \%$ in gyp1 $\Delta$ cells (Fig. 4.9D). Atg1 has been reported to interact preferentially with the GTP-bound form of Ypt1 (Wang et al., 2013). This interaction might be stabilized after deleting the respective GAP, GYP1 that causes the formation of Ypt1-GDP. Gyp1 might be needed for proper disassembly of the Ypt1-Atg1 complex. 
A

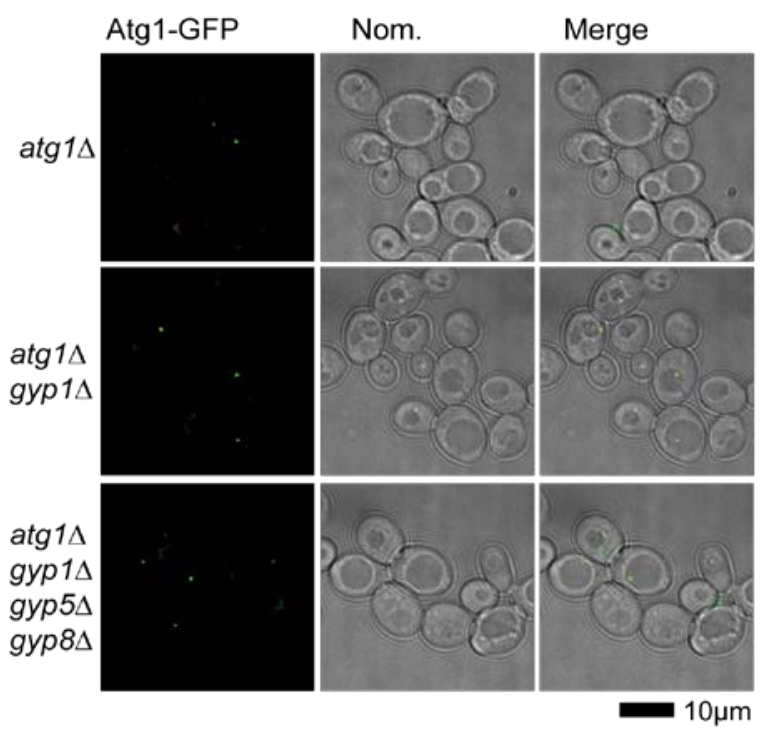

C

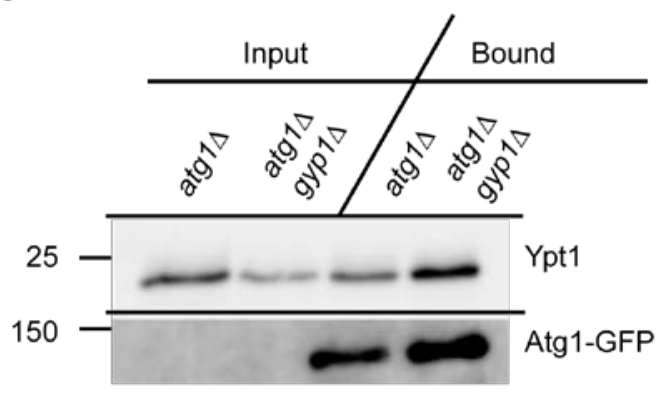

B

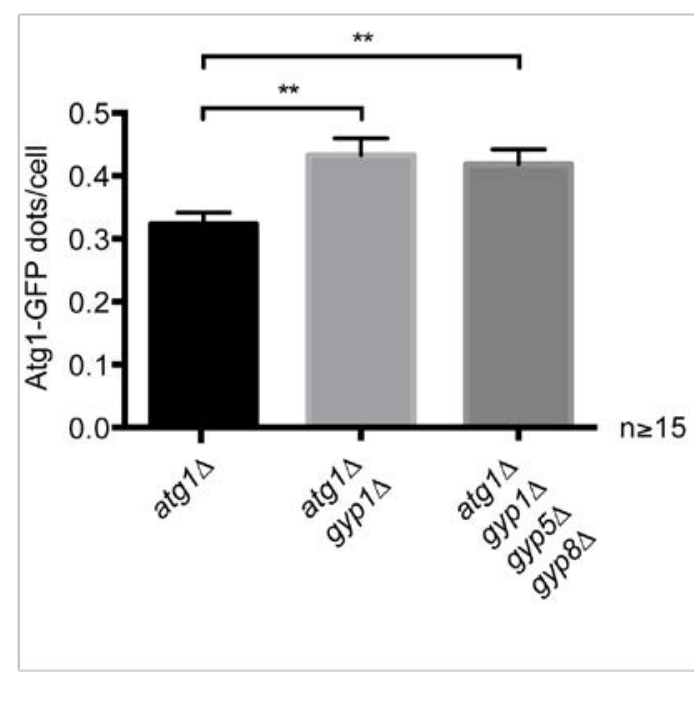

D

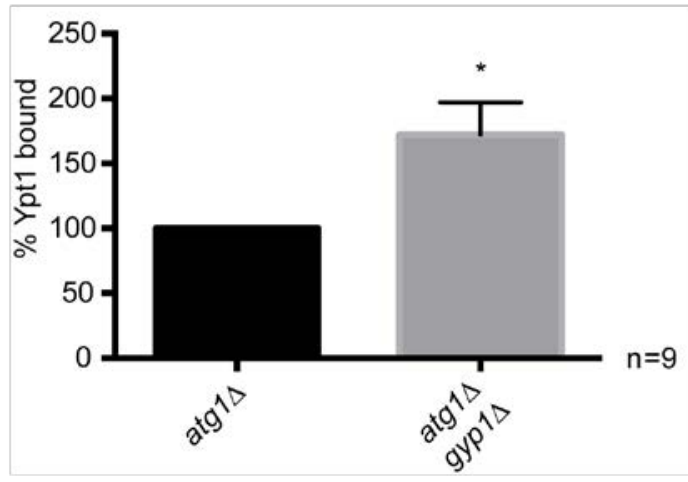

Figure 4.9: Analysis of Atg1-GFP in the absence of Gyp1

(A) ATG1-GFP was expressed on a plasmid from its endogenous promoter in $\operatorname{atg} 1 \Delta$, atg1 $1 \Delta y p 1 \Delta$ and $\operatorname{atg} 1 \Delta$ gyp $1 \Delta$ gyp5 gyp $8 \Delta$ cells and visualized by fluorescence microscopy. Cells were grown to logarithmic phase.

(B) Deletion of GYP1 as well as additional deletion of GYP5 and GYP8 led to an increase in Atg1-GFP dots/cell. Statistics were performed using the unpaired two tailed t-test. $\mathrm{n}$ indicates the number of pictures used for the analysis. Pictures were taken in at least three independent experiments. Error bars represent SEM and asterisks represent $p$-values.

(C) By using the GFP-TRAP approach, the Ypt1-Atg1 complex was analyzed in the atg1 $1 \Delta y p 1 \Delta$ strain. Ypt1 was detected with a Ypt1 antibody, Atg1-GFP with a GFP antibody. Cells were grown to logarithmic phase. The molecular weight marker is depicted in $\mathrm{kDa}$.

(D) The amount of Ypt1 that co-precipitated with Atg1-GFP increased upon deletion of GYP1. Statistics were done using the one sample t-test from nine independent experiments (indicated by $n$ ). The error bar represents SEM and the asterisk represents a $p$-value. 


\subsubsection{Gyp1 is important for proper recruitment of Atg14 to the PAS}

After Atg1 recruitment, PAS formation proceeds by recruitment of the autophagyspecific class III PI3-kinase complex. A specific component of this complex is Atg14 (Kihara et al., 2001). Impaired disassembly of the Ypt1-Atg1 complex could affect the recruitment of Atg14, leading to decreased levels of PI3P at the PAS.

A

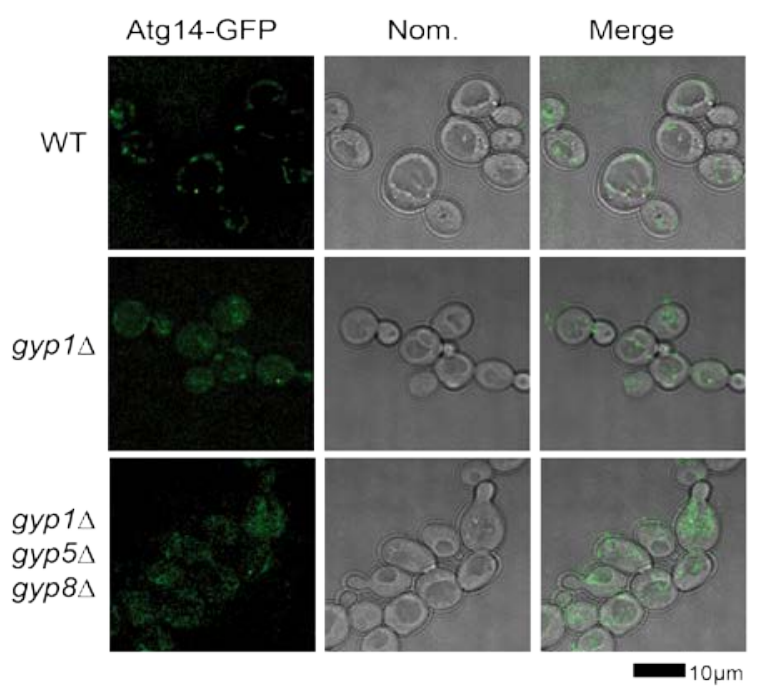

B

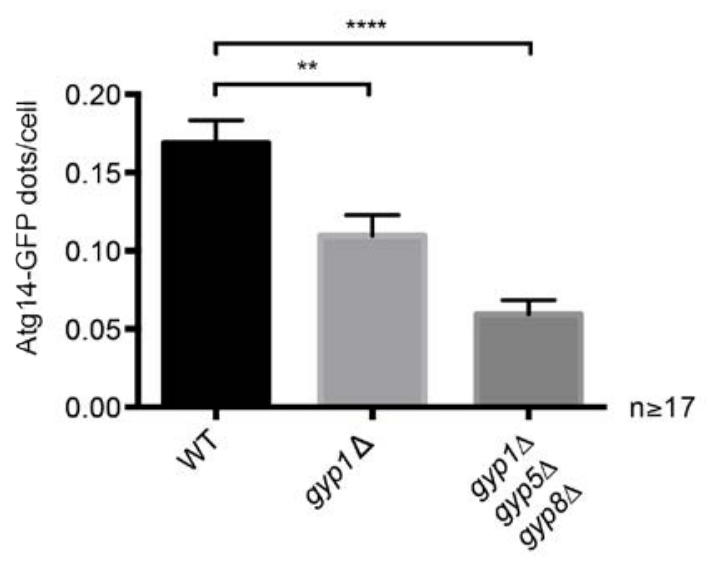

Figure 4.10: Atg14-GFP localization is affected upon GYP1 deletion

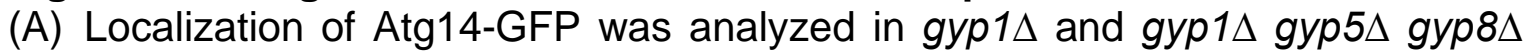
cells via fluorescence microscopy under logarithmic growth conditions. ATG14 was chromosomally tagged with GFP.

(B) Compared to the wild type, the number of Atg14-GFP dots/cell were significantly reduced in both deletions strains. Statistics were performed using the unpaired two tailed t-test. $\mathrm{n}$ indicates the number of pictures used for the analysis. Pictures were taken in at least three independent experiments. The error bars represent SEM and the asterisks represent $p$-values.

The localization of chromosomally tagged Atg14-GFP was examined in wild type,

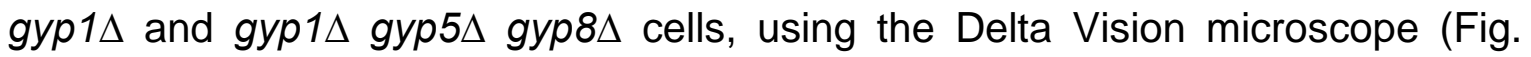
4.10A). The number of Atg14-GFP dots/cell was determined under logarithmic growth conditions. In gyp1s cells, the number of Atg14-GFP dots/cell was

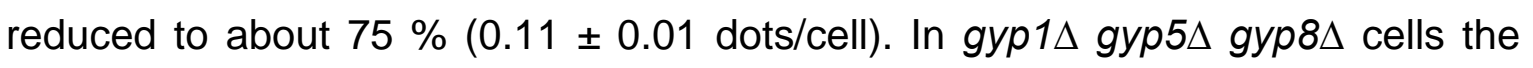
number of Atg14-GFP dots/cell was reduced to about $35 \%$ (0.06 0.01 dots/cell) (Fig. 4.10B). This indicates that Atg14-GFP is not properly located to the PAS in the absence of Gyp1. The defect in Atg14 recruitment is further increased in

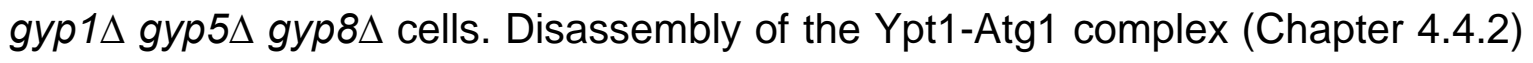
might be a prerequisite for proper Atg14 recruitment to the PAS. 
To further analyze recruitment of Atg14, its localization was examined by expressing the two GYP1 mutants on plasmids in chromosomally tagged ATG14-

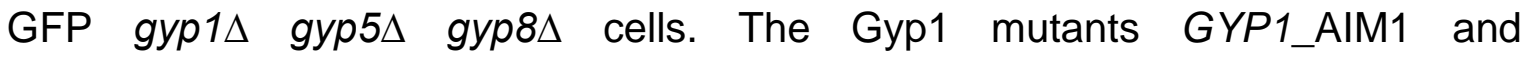
GYP1_R343K were plasmid-expressed from their endogenous promoters (Fig. 4.11A) and analyzed under logarithmic growth conditions. Gyp1_AIM1 (0.12 \pm 0.01 dots/cell) compared to Gyp1_WT (0.14 \pm 0.01 dots/cell) showed no significant reduction in the number of Atg14-GFP dots/cell (Fig. 4.11B). In cells containing the Gyp1_R343K mutant, the number of Atg14-GFP dots/cell was significantly reduced to $57 \%(0.08 \pm 0.01$ dots/cell).

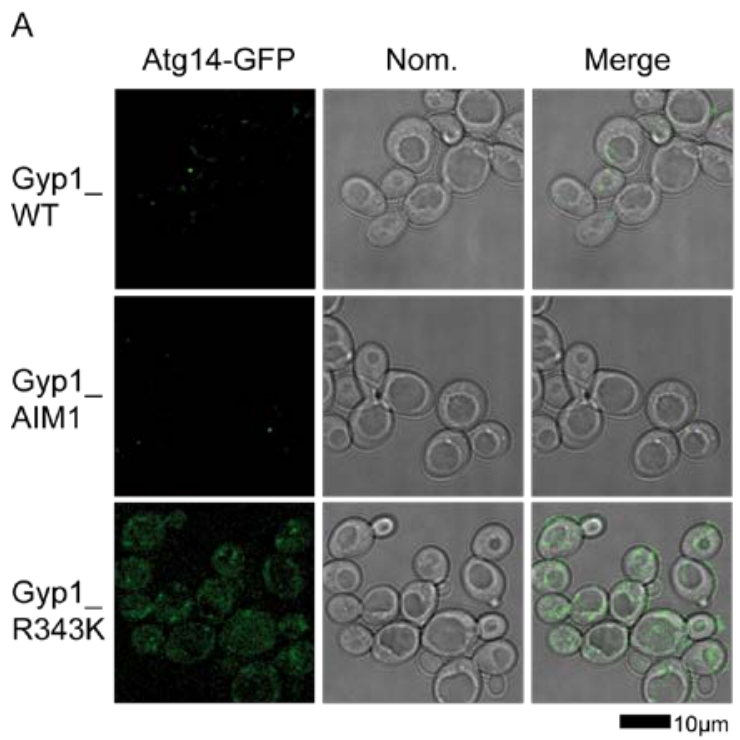

B

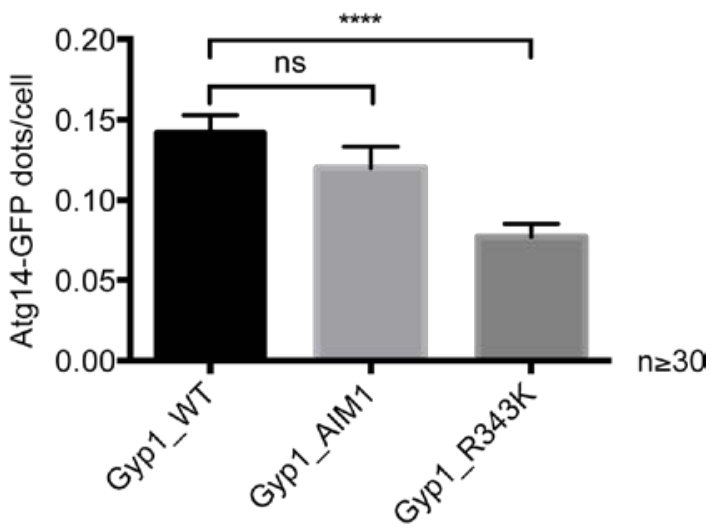

Figure 4.11: Ypt1-Atg1 complex disassembly is a prerequisite for proper Atg14 recruitment

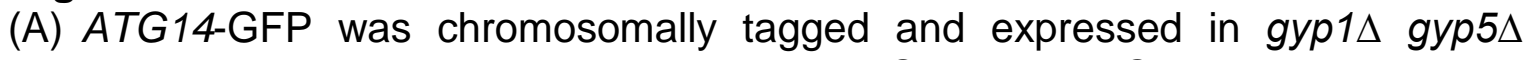
gyp8 cells together with the untagged GYP1_WT, GYP1_AIM1 and the GYP1_R343K plasmid and visualized by fluorescence microscopy. Cells were monitored under logarithmic growth conditions.

(B) A reduction in the number of Atg14-GFP dots/cell was observed in cells containing the Gyp1_R343K mutant and wild type-like levels were observed in cells containing Gyp1_AIM1 mutant. Statistics were done using the unpaired two tailed t-test and $\mathrm{n}$ indicates the number of pictures used for the analysis. Pictures were taken in at least three independent experiments. The error bars represent SEM and the asterisks represent p-values.

The exclusive reduction of the number of Atg14-GFP dots/cell in the Gyp1_R343K mutant cells indicates that Atg14-GFP recruitment depends on the GAP activity of Gyp1. The GAP activity of Gyp1 presumably plays a role in the efficient disassembly of Ypt1 and Atg1 (Chapter 4.4.2), which seems to be a prerequisite 
for Atg14 recruitment. Proper Atg14 localization is needed for Atg8 recruitment (Suzuki et al., 2007), which was examined in the next step.

\subsubsection{Gyp1 is needed for proper localization of Atg8 at the PAS}

The class III PI3-kinase complex, containing Atg14, is needed for further recruitment of proteins e.g. Atg8 to the PAS. Chapter 4.4.3 revealed that the recruitment of Atg14-GFP is reduced in cells containing the Gyp1_R343K mutant. The number of GFP-Atg8 dots/cell was determined under logarithmic growth conditions, by using the Delta Vision microscope (Fig. 4.12A).

A

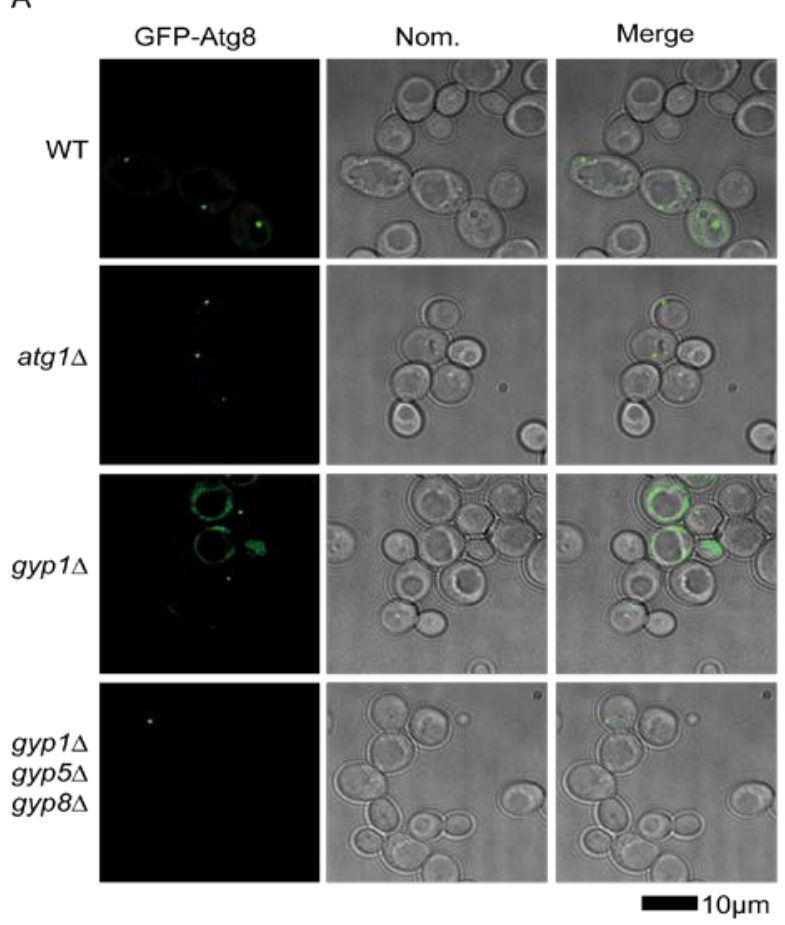

B

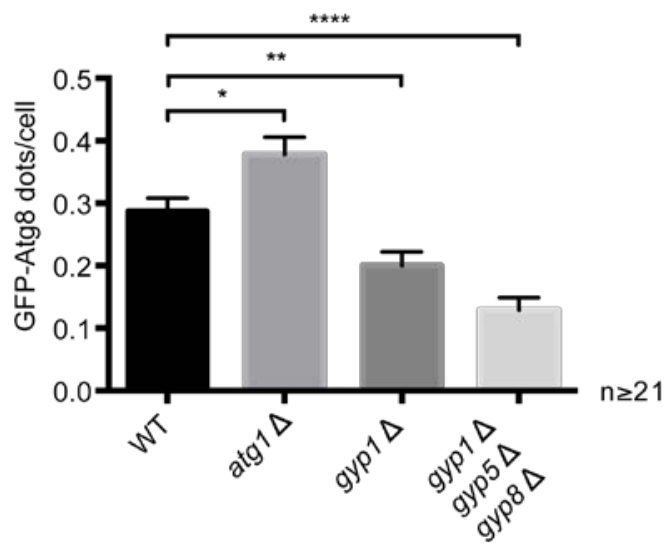

Figure 4.12: Proper Atg8 recruitment requires the Gyp proteins

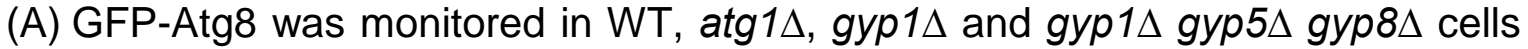
by using the Delta Vision microscope. Cells were analyzed under growth conditions.

(B) Deletion of GYP1 as well as deletion of GYP1, GYP5 and GYP8 caused a reduction in the number of GFP-Atg8 dots/cell. The deletion strains were compared to the WT strain using the unpaired two tailed t-test. $\mathrm{n}$ indicates the number of pictures used for the analysis. Pictures were taken in at least three independent experiments. Error bars represent SEM and the asterisks represent $p$-values.

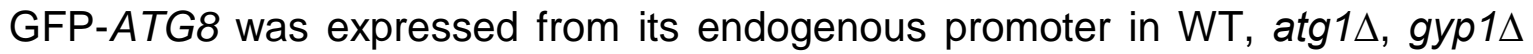

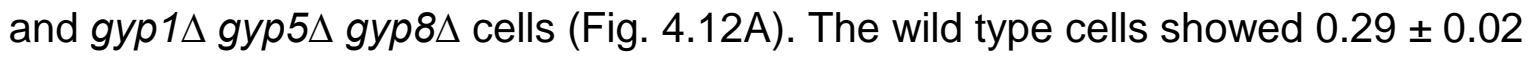
GFP-Atg8 dots/cell. gyp1 1 cells (0.2 \pm 0.02 , dots/cell; $69 \%$ compared to WT) as 
well as gyp1 $1 \Delta$ gyp5 gyp8 8 cells ( $0.13 \pm 0.02$ dots/cell; $45 \%$ compared to WT) displayed a reduction in the number of Atg8 dots/cell (Fig. 4.12B). The atg1 $\Delta$ strain, used as a control showed an expected increase in the number of GFPAtg 8 to $0.38 \pm 0.03$ dots/cell (Fig. 4.12B). This indicates that proper localization of Atg8 depends on the presence of Gyp1.

Whether this Atg8 localization depends on the GAP activity or on the interaction of Atg8 with Gyp1 was examined by expressing Cherry-GYP1_WT, Cherry-

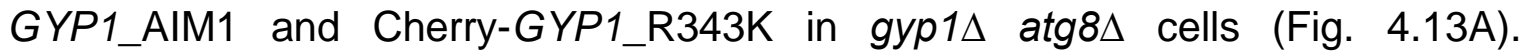
Furthermore, untagged GYP1_WT, GYP1_AIM1, GYP1_R343K and an empty plasmid were expressed in gyp $1 \Delta$ gyp5 $5 y$ gy $8 \Delta$ atg $8 \Delta$ cells (Fig. 4.13C).

In gyp1 1 atg $8 \Delta$ cells containing the Cherry-Gyp1_WT, $0.33 \pm 0.02$ GFP-Atg8 dots/cell were counted (Fig. 4.13B). The number of GFP-Atg8 dots/cell in gyp1 $\Delta$ atg $8 \Delta$ cells was not significantly decreased in the presence of the CherryGyp1_AlM1 mutant $(0.23 \pm 0.02$ dots/cell, $86 \%)$ compared to the CherryGyp1_WT (0.27 \pm 0.02 dots/cell, set to $100 \%)$. Only the strain containing CherryGyp1_R343K showed a significant reduction in the number of GFP-Atg8 dots/cell with $0.14 \pm 0.01$ dots/cell, which corresponds to a reduction of about $52 \%$ compared to Cherry-Gyp1_WT (Fig. 4.13B). 
A

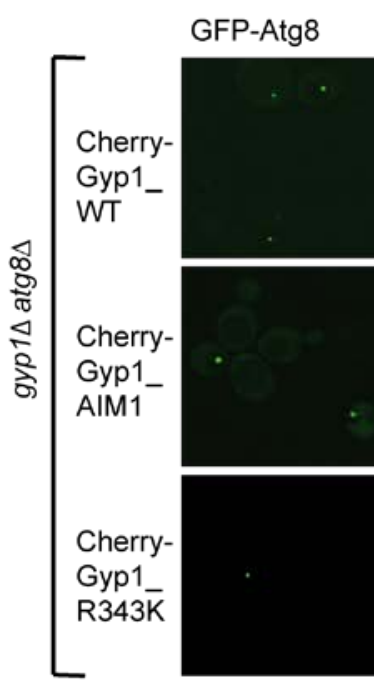

C

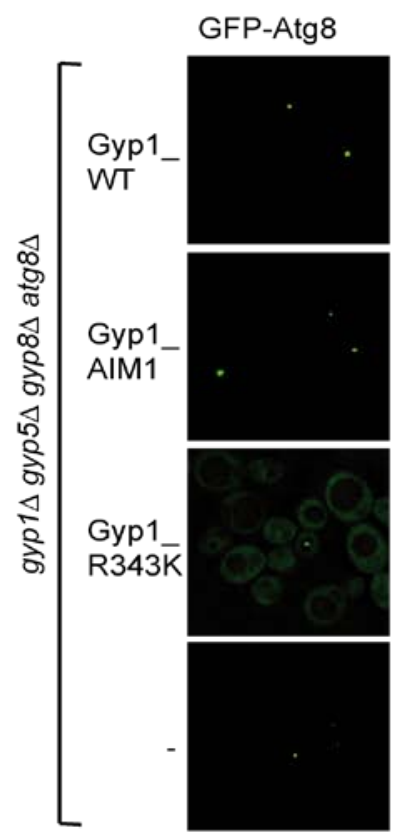

Nom.

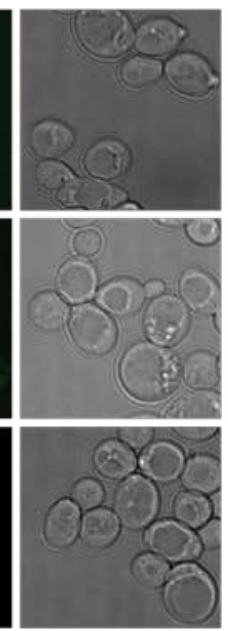

Merge

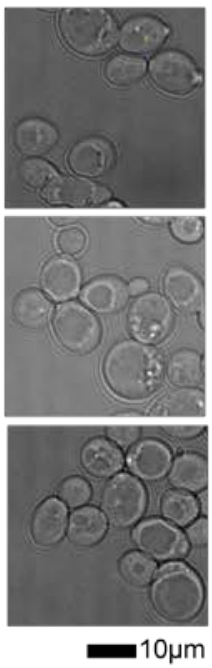

$-10 \mu \mathrm{m}$

\section{Nom.}
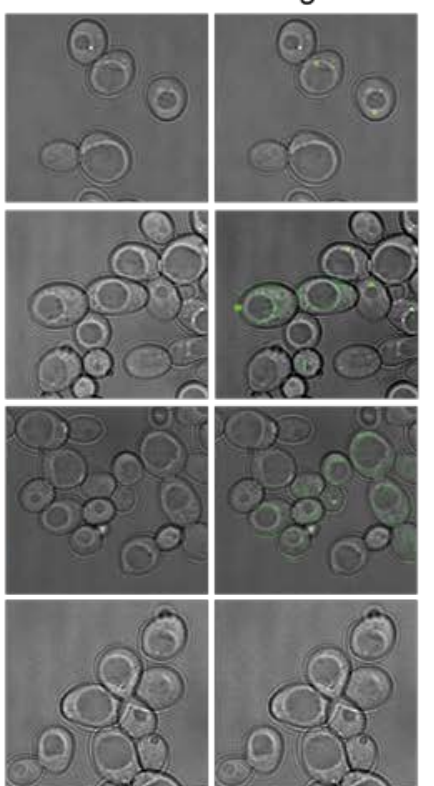

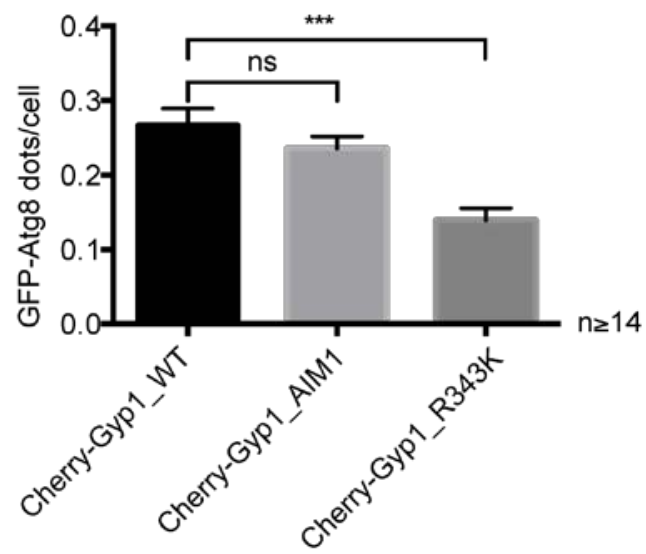

$\mathrm{D}$

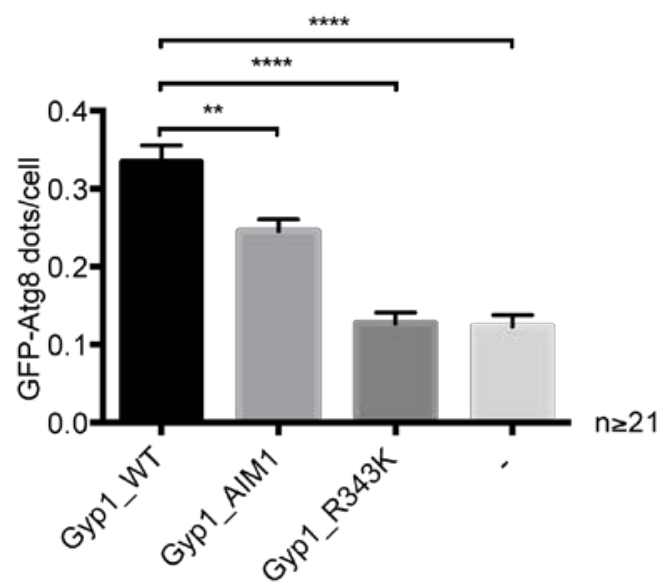

Figure 4.13: The GAP activity of Gyp1 and its interaction with Atg8 are needed for proper Atg8 localization at the PAS

(A) The localization of GFP-Atg8 was examined under logarithmic growth conditions in atg8 $8 y$ gy $1 \Delta$ cells, using the Delta Vision microscope.

(B) GFP-ATG8 was expressed together with Cherry-GYP1_WT, CherryGYP1_AIM1 or Cherry-GYP1_R343K in the gyp1D atg8 $\Delta$ strain. Cherry-GYP1 constructs were expressed from the MET25 promoter; GFP-ATG8 was expressed from its endogenous promoter. The number of GFP-Atg8 dots/cell was reduced in the strain containing the Cherry-Gyp1_R343K and showed WT-levels in the strain containing Cherry-Gyp1_AIM1. Statistics were done using the unpaired to tailed t-test. $\mathrm{n}$ indicates the number of pictures used for the analysis. Pictures were taken in at least three independent experiments. The error bars represent SEM and the asterisks represent $p$-values. 
(C) GYP1 (untagged) and GFP-ATG8 plasmids were expressed from their endogenous promoters in the gyp1 $1 \Delta$ gyp5 $5 y p 8 \Delta$ atg $8 \Delta$ strain and the localization of GFP-Atg8 was analyzed. Cells were monitored under logarithmic growth conditions.

(D) The number of GFP-Atg8 dots/cell was reduced in the two strains containing the Gyp1_AIM1 and Gyp1_R343K mutants. Statistics and analysis were performed as indicated in (B).

In gyp1 1 gyp5 gyp8 8 atg8 8 cells (Fig. 4.13C), the presence of Gyp1_AlM1 led to a decrease in the number of GFP-Atg8 dots/cell to $0.25 \pm 0.02$ or about $76 \%$ compared to wild type Gyp1 (0.33 \pm 0.02 dots/cell, set to $100 \%$ ) (Fig. 4.13D). The Gyp1_R343K mutant lacking the GAP activity of Gyp1 caused a further decrease in the number of GFP-Atg8 dots/cell to $0.13 \pm 0.01$ or $39 \%$, which is almost the amount of GFP-Atg8 dots/cell in the GYP1 deletion cells with $0.12 \pm 0.02$ dots/cell (36\%).

Thus, the Gyp1_R343K mutant has an effect on GFP-Atg8 localization in both deletion strains, whereas the Gyp1_AIM1 mutant only leads to a decreased number of GFP-Atg8 dots/cell upon additional deletion of GYP5 and GYP8.

\subsection{Analysis of Atg8 binding to specific cargo receptors}

Analysis of Atg14-GFP (Chapter 4.4.3) revealed that the Gyp1_AlM1 mutant does not impair Atg14-GFP recruitment to the PAS. Thus, it is likely that the PI3P levels are normal in this mutant. The results also showed that the number of Atg8 dots/cell at the PAS was wild type-like in the Gyp1_AIM1 mutant (Chapter 4.4.4). Thus, the function of Gyp1_AlM1 might be needed for later steps of autophagy after proper recruitment of Atg8. In later steps of autophagy, Atg8 interacts with cargo receptors. Thus, the interaction of Atg8 to the cargo receptors Atg19 (Cvtpathway) and Atg32 (mitophagy) was investigated next.

\subsubsection{Interaction of Atg8 and the cargo receptor for mitophagy Atg32 depends on Gyp1}

The binding of Atg32, the cargo receptor for mitophagy, to Atg8 was analyzed using the GFP-TRAP approach. For this, GFP-ATG32 and 2xFlag-ATG8 were

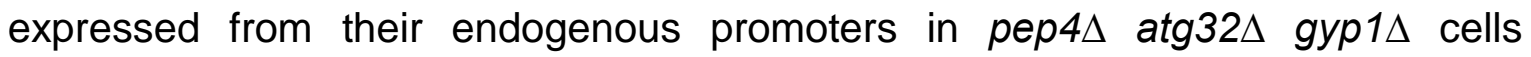
together with the GYP1_WT, GYP1_AIM1 and the GYP1_R343K mutant plasmids 
or an empty plasmid. The cells were shifted to $2 \%$ lactate medium and incubated for $24 \mathrm{~h}$ to $30 \mathrm{~h}$ to induce mitophagy. Figure 4.14A represents western blot analysis of 2xFlag-Atg8 after high and low exposure. To differentiate the input fraction of Flag-Atg8, low exposure conditions were used. Visibility of the bound fraction was enhanced by high exposure conditions. The amount of Gyp1_WT was set to $100 \%$. In cells containing the Gyp1_AIM1 mutant, binding of 2x-FlagAtg8 to GFP-Atg32 was decreased to $68.95 \pm 11.71 \%$, in the Gyp1_R343K mutant to $49.95 \pm 7.35 \%$ and in the gyp1s to $64.10 \pm 8.38 \%$. Thus, Gyp1 might be involved the binding of Atg8 to cargo receptors. The Atg8-Atg32 interaction in the Gyp1_R343K mutant is most likely affected due to the deficient dissociation ability of the Ypt1-Atg1 complex, which already affected earlier steps of autophagy.
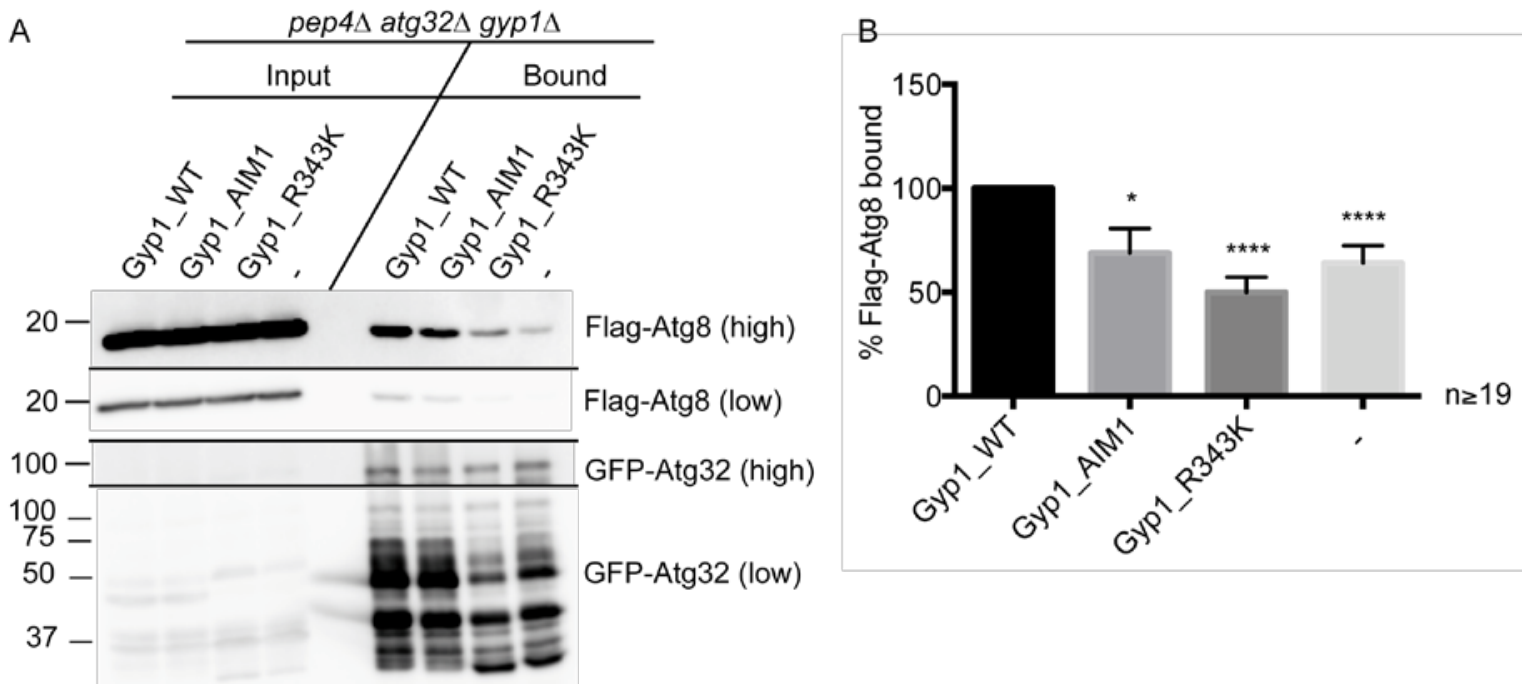

Figure 4.14: The interaction of Atg8 and Atg32 is reduced upon deletion of GYP1

(A) The interaction of Atg8 and the mitophagy receptor Atg32 was analyzed under mitophagy-inducing conditions using a GFP-TRAP approach. A Flag antibody was used to detect 2xFlag-Atg8; a GFP antibody for GFP-Atg32. Protein interaction was analyzed in pep $4 \Delta$ atg $32 \Delta$ gyp $1 \Delta$ cells. The molecular weight marker is depicted in $\mathrm{kDa}$.

(B) The interaction of Atg8 and Atg32 is significantly reduced in the pep4 $\Delta$ atg $32 \Delta$ gyp1 $1 \Delta$ cells. Compared to the wild type Gyp1, Gyp1_AIM1 and Gyp1_R343K, impaired the Atg8-Atg32 interaction. Statistics were done using the one sample t-test for 19 independent experiments. The error bars represent SEM and the asterisks represent $p$-values. 


\subsubsection{Binding of Atg8 to the cargo receptor Atg19 is reduced upon GYP1 deletion}

Gyp1 might play a more general role in the process of cargo recognition by Atg8. Therefore, after showing a reduced interaction of Atg8 and Atg32 in gyp1 1 cells, (Chapter 4.5.1) another receptor for autophagy was tested. Atg19 is the receptor protein for the Cvt-pathway and required for the transport of the hydrolases Ape1 and Ams1 to the PAS where the Cvt-vesicles are formed. The split-ubiquitin assay was used to examine the binding of Atg19 and Atg8 after GYP1 deletion. Atg19 was fused to the C-terminal and Atg8 to the N-terminal half of ubiquitin. Plasmidencoded GYP1 was expressed untagged from its endogenous promoter in wild type and gyp1 $1 \Delta$ cells. The proteins Ste14 and Ubc6 were used as control and analyzed in wild type cells. The indicated strains were spotted in 10 -fold dilution steps on plates for growth control (-Ura -Leu -His) and selection (+FOA and -Ura). The control cells containing Ste14-Cub and Nui-Ubc6 as positive control and Ste14-Cub with an empty vector as negative control showed the expected growth pattern (Fig. 4.15). Cells of the positive control grew on +FOA plates but not on Ura plates. Cells expressing only Ste14, therefore lacking the N-terminal part of ubiquitin, grew on -Ura plates but not on +FOA plates (Müller and Johnsson, 2008).

\begin{tabular}{|c|c|c|c|c|c|c|c|c|c|c|c|c|}
\hline Cub & Nub & Gyp1 & strain & -Trp -His -Le & & & $+\mathrm{F}$ & & & & & \\
\hline Ste14 & Ubc6 & - & WT & $0<\Delta$ & : & 0 & 0 & 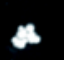 & 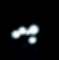 & & & \\
\hline Ste14 & - & - & WT & 006 & 8 & $\bullet$ & 6 & & & & s & \% \\
\hline Atg19 & Atg 8 & WT & 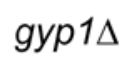 & $00 \%$ & 3 & ○. & 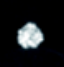 & * & $\cdot$ & 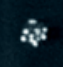 & $\therefore$ & - \\
\hline $\operatorname{Atg} 19$ & Atg8 & - & 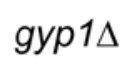 & 00 & $\bullet$ & 0 & $\%$ & $\because$ & . & s & $\because$ & . \\
\hline Atg19 & - & - & gyp1А & $00 \%$ & 8 & & & & & 0 & & 2 \\
\hline
\end{tabular}

Figure 4.15: Binding of Atg8 to Atg19 in the absence of Gyp1 analyzed by the split-ubiquitin assay

The respective strains were spotted on plates for growth control (-Ura -Leu -His) and selection (+FOA and -Ura) in 10-fold dilution steps. The proteins Ste14 and Ubc6 were used as control and analyzed in wild type cells. The interaction of Atg8 and the receptor for the Cvt-pathway, Atg19, is reduced in the GYP1 deletion strain (lane 4) compared to Gyp1_WT (lane 3) indicated by growth on the +FOA plate or the -Ura plate. 
Visual evaluation of the split-ubiquitin assay revealed a reduced binding of Atg8 and Atg19 in gyp1A cells compared to cells expressing the GYP1_WT (Fig. 4.15). These results further support the involvement of Gyp1 in the Cvt-pathway. Here Gyp1 might influence the binding of Atg8 to the cargo-receptor Atg19, comparable to its role in the interaction of Atg8 and Atg32 during mitophagy. The observed phenotype might also be caused by the defect in disassembly of the Ypt1-Atg1 complex and needs further investigation. 


\section{Discussion}

Autophagy is an important process for degradation and recycling of cellular components to maintain cellular homeostasis. It is evolutionary conserved and characterized by the de novo formation of a unique double-membrane layered vesicle, the so-called autophagosome that transports its enclosed contents to the vacuole for degradation. Autophagy is initiated by assembly of the autophagic machinery at the PAS. Here, the phagophore, a double-layered membrane structure is formed that elongates to engulf cytoplasmic components or organelles. Upon closure of the phagophore around its targets, the structure is termed autophagosome (Feng et al., 2013; Reggiori and Klionsky, 2013; Farré and Subramani, 2016). The degradation processes involve vacuolar hydrolases, which also need to be transported to the vacuole. One transport pathway for hydrolases also utilizes the autophagic machinery and is termed the Cvt-pathway (Lynch-Day and Klionsky, 2010). Initially, autophagy has been defined as a nonselective degradation pathway activated by nutrient depletion and stress. Knowledge about autophagy and its broad cargo spectrum increased over the past decades and revealed new insights into selective forms of autophagy (Zaffagnini and Martens, 2016). Today, various autophagic cargo receptors are identified that target intracellular protein aggregates, organelles and pathogens to the vacuole in yeast or the lysosome in mammals for degradation.

Over the past years, the role of Ypt/Rab proteins as autophagy regulators became more and more evident (Kern et al, 2015). More than 70 Rab proteins are identified in mammals and about ten of them have been shown to participate also in autophagy (Szatmári and Sass, 2014). Ypt/Rab proteins contain a conserved nucleotide-binding domain to bind GTP (activation) as well as GDP (inactivation). Active $Y p t / R a b$ proteins are attached to the membrane, to which they recruit their effector proteins. The inactive Ypt/Rab proteins are soluble in the cytoplasm and membrane binding is prevented by GDP dissociation inhibitors (GDIs). Ypt/Rabs can be activated by nucleotide exchange factors (GEFs) that simulate the exchange of GDP to GTP. Inactivation is stimulated by GTPase-activating proteins (GAPs) (Bos et al., 2007; Nottingham and Pfeffer, 2009). The PAS is a highly dynamic protein complex with numerous proteins that assemble and disassemble to the structure. Formation of the phagophore needs the supply of 
membranes and proper vesicle transport. Over the last years, GTPases attracted increasing attention also in the field of autophagy, as they are key regulators in vesicle traffic (Kern et al., 2015; Hutagalung and Novick, 2011).

Ypt1 is a GTPase and known to play a role in autophagy by regulation of Atg1 and Atg11 and involvement in Atg9 vesicle trafficking. Atg1 and Atg11 interact with Ypt1 preferentially in its GTP-bound form (Lipatova et al., 2012; Wang et al., 2013). One GAP of Ypt1 is Gyp1, a cis-Golgi GTPase-activating protein and has been reported to inactivate only Ypt1 in vivo, but a variety of proteins in vitro namely Sec4, Ypt7 and Ypt51 (Du et al., 1998). Gyp1 was identified as a potential interaction partner of Atg8 by mass spectrometry. This study proposes Gyp1 as a potential regulator of autophagy that seems to be important for two different steps during autophagosome formation.

\subsection{Identification of Gyp1 as an Atg8 interaction partner}

Gyp1 was formerly identified as a potential interaction partner of Atg8 by mass spectrometry analysis (Dr. R. Krick, personal communication, 2015). Gyp1 is known for its role as a GAP for Ypt1. The GTP hydrolysis of Ypt1 is mediated preferentially by Gyp1 in vivo but also by two other GAPs Gyp5 and Gyp8. These three GAPs are located to different compartments in the cell (Du and Novick; Lafourcade et al., 2004), but as they have an overlapping function in Ypt1 inactivation, they were included into most analyses. By using a GFP-TRAP approach, it was shown that Atg8 preferentially interacts with Gyp1 whereas binding to Gyp5 and Gyp8 was only weak (Fig.4.1A and B).

This study shows that the interaction of Gyp1 with Atg8 is mediated by the AIMbinding sites in Atg8 (Fig. 4.2A and B) and that Gyp1 binds to Atg8 in an AIMdependent manner (Fig. 4.4A and B). For Gyp1, the iLIR software predicted seven AIMs (Fig. 4.3), but only one AIM mutant (AIM1 $\mathbf{W}^{160}$ SAV ${ }^{163}$ ) clearly affected binding to Atg8. This results point to a direct interaction of both proteins. Mutation of Gyp1_AIM3 leads to an increase in binding of Gyp1 to Atg8 to $177 \%$ (Fig. 4.4 A and B). Mutating this motif might increase the affinity of Gyp1 to Atg8. Gyp1 might also bind to another interaction partner via this motif and binding of this protein is a prerequisite for dissociation of Atg8. Gyp1_AIM1 and Gyp1_AIM3 are furthermore the only mutant proteins, which are not reduced in their protein 
level detected by western blot (Fig. 4.4C). The instability of the other mutant proteins avoided their analysis as potential AIMs. The AIM mutants might be destabilized because the mutated amino acids contribute to protein stability, proper Gyp1 localization in the cell or its interaction with other proteins. Nevertheless, it might be still possible that these predicted AIMs contribute to the interaction of Gyp1 with Atg8. This might be further analyzed under more physiological conditions e.g. in intact cells. Furthermore, it is possible that the iLIR software might have not predicted all potential AIMs in Gyp1. The existence of additional AIMs in Gyp1 could also explain the lack of phenotype in the Cvtpathway or the partial defect in mitophagy. For example, the Cvt receptor Atg19 contains multiple cryptic AIMs that contribute to the Atg19-Atg8 interaction (SawaMakarska et al., 2014).

Gyp1 might interact with Atg8 additionally via the F5K6 site in Atg8. Mutating these residues reduced binding of Gyp1 to Atg8 to $43 \%$ compared to wild type (Fig. 4.2B). The FK-motif is part of a flexible arm in Atg8 and might interact directly with Gyp1 or support the interaction indirectly. The R343 site is crucial for the GAP activity of Gyp1 but not for its binding to the substrate Ypt1 (Du and Novick, 2000). Mutating the R343 site (to Gyp1_R343K) in Gyp1 does not influence binding of Gyp1 to Atg8 (Fig. 4.4E and D).

The GFP-TRAP, for identification of the binding sites, was performed using different Atg8 protein concentrations. The experiment was either performed using GFP-ATG8 expressed from a GAL1 (pYES2 plasmid) promoter (leading to overexpression of the protein) or using GFP-ATG8 expressed from a low-copy plasmid under its endogenous promoter, leading to more native protein concentrations. Both approaches were performed to compensate the particular disadvantages of each experimental setup. Overexpressed GFP-Atg8_L50A leads to a reduction to $9 \%$ in binding of Gyp1-HA (Fig. 4.2B), indicating that the GFP-Atg8 protein concentration is high enough to saturate the GFP-TRAP beads. In the experiment using the endogenous ATG8 promoter, binding of Gyp1 to Atg8_L50A is reduced to $38 \%$ compared to wild type (Fig. 4.2D).

The surface of the GFP-beads consists of a GFP-binding protein, which is supposed to interact with the GFP-tag attached to the protein of interest. The GFP-TRAP beads have to be saturated with GFP, otherwise proteins might attach 
unspecifically to the surface of the beads. Unspecific binding can be observed when an empty plasmid without GFP-tag is expressed in the cell or when the concentration of the GFP-tagged protein is low. This can be a reason for the more obvious reduction in binding of Atg8_L50A and Gyp1 in the experiments using the GAL1 promoter. The lower GFP-Atg8 concentration in the experiment with the endogenous promoter is not able to saturate the GFP-TRAP beads. This might lead to unspecific binding of Gyp1-HA to the beads.

For the experiment with the overexpressed GFP-ATG8, a negative control was used. The GFP-tag alone was expressed form the GAL1 promoter and is able to interact with the GFP-TRAP beads. Thereby, unspecific binding of proteins to the GFP-TRAP beads is avoided and unspecific binding to the GFP-tag alone can be analyzed. Such a negative control was not used for the experiment with endogenous concentrations of GFP-Atg8 and could be included into further experiments. Furthermore, this experiment might be optimized by e.g. by using higher protein amounts to reduce the presumed background caused by unspecific protein interactions with the beads.

In the GFP-TRAP approach, other proteins are still present in the yeast cell lysate that is incubated with the GFP-TRAP beads. Another Atg8 interaction partner might bridge the interaction of Atg8 and Gyp1 and the detected interaction might therefore not be direct. Atg8 is known to interact with Atg1 in an AIM-dependent manner (Nakatogawa et al., 2012b) and Atg1 is known for its interaction with Ypt1 at the PAS, which is likely regulated by Gyp1 (Lipatova et al., 2012; Kramer et al., 2017). Thus, Atg8 and Gyp1 are likely together at the PAS and Ypt1/Atg1 could be candidates to bridge their binding. The identification of an AIM points to a direct interaction of Gyp1 and Atg8.

Only one AIM-containing protein can bind to Atg8 in the same complex. AIMcontaining proteins compete for the AIM binding site in Atg8 and thereby exclude their simultaneous binding as it has been shown for Atg32, which competes with Atg12-Atg5 for Atg8-PE interaction (Kaufmann et al., 2014). During autophagy, Atg8 dependent interactions link a variety of proteins to the phagophore. Atg8 thereby not only binds the cargo receptors to the inner side of the phagophore but also interacts with the scaffold complex on the outside of the phagophore (Atg12), its conjugation machinery (Atg3 and Atg4) or Atg1 (Kraft et al., 2012; Nakatogawa 
et al., 2012b; Yamaguchi et al., 2010; Abreu et al., 2017; Kaufmann et al., 2014). Thus the AIM-binding sites of Atg8 are required for the various functions of Atg8.

There are various different approaches to detect AIMs and to enhance the accuracy of their determination (Popelka and Klionsky, 2015; Kalvari et al., 2014; Dinkel et al., 2014). In Gyp1, seven potential AIMs were predicted, indicating a frequent occurrence of this motif in Gyp1. AIMs have also been identified in a high frequency in other Atg proteins, using different approaches for their identification (Popelka and Klionsky, 2015). Thus, Atg2 has 22 predicted AIMs, Atg5 has six and Atg20 has five AIMs using the ELM algorithm. Atg32 has a predicted AIM in its intermembrane space region, which is unlikely to bind cytosolic Atg8 (Popelka and Klionsky, 2015). None of these predicted AIMs have so far been identified as functional. Thus, the prediction of an AIM alone is not sufficient to identify an interaction partner of Atg8.

A possibility to confirm the direct interaction of Atg8 and Gyp1 is to eliminate all interaction partners that could bridge the binding of Gyp1 and Atg8. This was achieved by performing a recombinant pulldown assay by Dr. Krick (personal communication, 2015). His-Gyp1 and GST-Atg8 were purified from in E. coli and used for an interaction analysis in the absence of further proteins (according to the protocol in Juris et al., 2015). This assay demonstrated that both proteins interact directly and that this interaction is mediated by the AIM1 in Gyp1 (HisGyp1_AIM1 showed only $3 \%$ binding to GST-Atg8 compared to wild type). Data of these experiments are not presented in this thesis but should be considered as additional information as they support a direct and AIM-dependent interaction of Gyp1 and Atg8. Taken together, these experiments show a direct interaction of Gyp1 and Atg8 in an AIM-dependent manner.

This study identified Gyp1 as a novel interaction partner of Atg8. Binding of the two proteins is mediated in an AIM-dependent manner and mutation of the respective residues either in Gyp1 or Atg8 leads to reduced interaction levels. This interaction is independent of the R343 site in Gyp1, which is important for its GAP activity. Further aims might be the optimization of the GFP-TRAP approach with endogenous protein concentrations and the identification of additional AIMs 
or regulatory motifs that influence the interaction of Gyp1 and Atg8. To further investigate the role of Atg8-Gyp1 interaction, proceeding of selective autophagy was examined.

\subsection{Gyp1 contributes to selective forms of autophagy}

The putative role of Gyp1 in autophagy was examined by monitoring pApe1

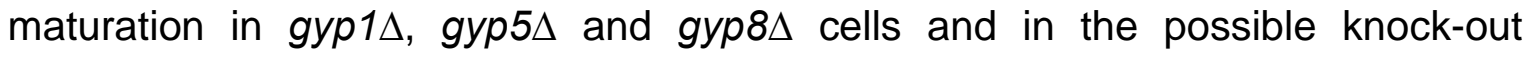
combinations. The results of these experiments reveal a function of Gyp1 in the Cvt-pathway, as maturation of pApe1 is reduced to about $75 \%$ in the GYP1 deletion cells. Single deletion of GYP5 and GYP8 has no effect on pApe1 maturation, but deletion of the proteins in addition to GYP1 caused a reduced pApe1 maturation rate. Deletion of all three GAPs leads to the most severe phenotype with a reduction in pApe1 maturation to $28 \%$ (Fig. 4.5A and B). As single deletion of GYP5 and GYP8 do not show a defect in pApe1 maturation, Gyp1 might be the major GAP for the Cvt-pathway. Additional deletion of GYP5 and GYP8 in gyp1 $1 \Delta$ cells increased the defect in pApe1 maturation and thus the two GAPs might be able to replace at least partially the function of Gyp1.

The three GAPs were reported to stimulate GTP hydrolysis of Ypt1 in vivo (Laufouraced et al., 2004; De Antoni et al., 2002). In the absence of these GAPs, Ypt1 still has a low intrinsic GTP hydrolysis rate of $0.002 \mathrm{~mol}$ of GTP per mol of Ypt1/min at a temperature of $30{ }^{\circ} \mathrm{C}$. Saturating the Ypt1-GAP activity leads to an increase to $0.108 \mathrm{~mol} / \mathrm{mol} / \mathrm{min}$ (Richardson et al., 1998). Thus, the residual intrinsic GTP-hydrolysis of Ypt1 is also present upon deletion of all three GAPs and might lead to the slight pApe1 maturation in the triple knockout cells in vivo. Although it cannot be excluded yet, that there are other GAPs that also stimulate Ypt1.

The observed pApe1 maturation phenotype was detected under nutrient-rich conditions and was absent after four hours of nitrogen starvation (Fig. 4.5C and D). A study from Kramer et al. (2017) also reported a pApe1 maturation phenotype under nutrient-rich conditions, which is in line with the findings of this study. The authors furthermore show a gyp1s phenotype under nitrogen starvation measured by the Pho8 $\Delta 60$ assay. This assay measures the activity of the alkaline phosphatase Pho8 with a modified C-terminal region. The protein 
becomes soluble in the cytosol due to the modification and can be used to monitor nonselective autophagy (Noda and Klionsky, 2008).

In this study, a pApe1 maturation phenotype was absent under starvation conditions (Fig. 4.5C and D). The pApe1 maturation assay might be not a suitable method to determine an autophagy phenotype under nitrogen starvation. During the Cvt-pathway, pApe1 is closely surrounded by the Cvt-vesicle and cytoplasm is excluded. Upon starvation, the transport of pApe1 is taken over by macroautophagy and pApe1 is engulfed by the much larger autophagosome together with other materials destined for degradation. Expression of pApe1 is induced 19-fold upon starvation and so is the capacity for its sequestration (Scott et al., 1996). Thus, the mechanism of pApe1 transport to the vacuole differs depending on the nutrient supply. Gyp1 might be needed only under nutrient-rich conditions or the phenotype is masked due to higher amounts of pApe1 under starvation conditions. Furthermore, pApe1 maturation was measured after $4 \mathrm{~h}$ starvation. Here, pApe1 transport to the vacuole and its maturation might have reached a final level and pApe1 might be fully processed. Thus, further studies might include the analysis of pApe1 maturation after a shorter period of starvation or using a pulse-chase assay.

The role of Gyp1 in pApe1 maturation was further examined under nutrient-rich conditions. Therefore, GYP1_WT and its two mutants GYP1_R343K and GYP1_AIM1 were expressed from a low copy plasmid and pApe1 maturation was analyzed by western blot. A reduction in pApe1 maturation was only detectable in the Gyp1_R343K mutant but not in the Gyp1_AIM1 mutant in gyp1s cells and gyp $1 \Delta$ gyp5 5 gyp8 $\Delta$ cells (Fig 4.5E and F). Thus, the GAP activity of Gyp1 seems to be important for the Cvt-pathway but not the interaction of Gyp1 and Atg8. The reduction of pApe 1 maturation is only reduced to about $75 \%$ in gyp $1 \Delta$ cells. Thus, measurement of a phenotype by expressing GYP1_AIM1 in the gyp1 $\Delta$ cells might be difficult based on the weak phenotype that is caused by complete deletion of

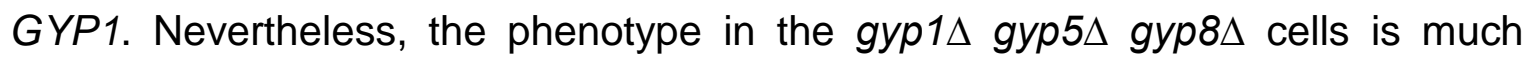
more severe (reduction to $28 \%$ mApe1) and is mainly caused by deletion of GYP1 as the gyp5 gyp8 cells show a pApe1 maturation rate of $99 \%$ (Fig. 4.5A and B). In these cells, a Gyp1_AIM1 phenotype was also not detectable. Possibly, Gyp1 possesses further AIMs that are used for the interaction with Atg8. For 
example, the Atg19-Atg8 interaction is mediated by several cryptic AIMs in Atg19 (Sawa-Makarska et al., 2014). Furthermore, the interaction might still occur also after only mutating the AIM and a deletion of the complete AIM might lead to an observable effect. This hypothesis needs further investigation. Based on the present data, the GAP activity of Gyp1 seems to be important for the Cvt-pathway under nutrient-rich conditions but not the interaction of Gyp1 and Atg8.

To verify a role of Gyp1 that is restricted to selective forms of autophagy, further assays should be performed. For example, the Pho8 $\Delta 60$ assay or measurement of PGK1 (3-phosphoglycerate-kinase)-GFP, a housekeeping protein, which is targeted to the vacuole and releases proteolytically resistant GFP that can be monitored by western blot analysis (Welter et al., 2010). A common method to monitor bulk autophagy is measurement of the degradation of GFP-Atg8 (Toggeler et al., 2017). As this protein is directly involved in the investigated process, the other two methods should be preferred. Kramer et al. (2017) further reported the interaction of Gyp1 with the Atg proteins Atg9, Atg18 (Atg9-recycling system) and Atg3, Atg5 and Atg7 (ubiquitin-like conjugation system). These interactions were monitored under starvation conditions and rapamycin treatment but not under nutrient-rich conditions. These findings highly support a role of Gyp1 also under starvation conditions and need further investigation.

Another selective form of autophagy is mitophagy, the degradation of mitochondria (Farre et al., 2009). The mitophagic rate was determined in the GYP1 deletion strain using Su9-DHFR fused to a proteolytic resistant GFP-tag. Upon mitophagy induction, this protein is transported to the vacuole and degraded, resulting in free GFP. Free GFP was detected on western blot. Deletion of GYP1 causes a reduction of the mitophagic rate to $51 \%$. Expression of the GYP1_AIM1 in gyp1D cells leads to a significant reduction to $80 \%$ compared to the Gyp1 wild type. The GYP1_R343K mutant also shows a reduced mitophagic rate of $62 \%$ (Fig. 4.6A and B). Thus, the GAP activity of Gyp1 and its interaction to Atg 8 seem to be needed for proper proceeding of mitophagy.

In the mammalian system, the GAP TBC1D15 has been reported to locate on mitochondria that are targeted for degradation and on the autophagosome 
(Yamano et al., 2014). TBC1D15 is thought to control the morphology of the autophagosome during mitophagy but not during unselective autophagy. TBC1D15 regulates the GTPase Rab7 and binds LC3 homologs in a LIRdependent manner. The authors concluded that the GAP activity of TBC1D15 forces the autophagosomal membrane to tightly enclose its cargo and furthermore inhibits Rab7 to release LC3 from microtubules and attach it to the cargo (Yamano et al., 2014). This demonstrates the function of a mammalian GAP in mitophagy and proposes a general role of GAP proteins in selective autophagy. Further studies of Gyp1 and its role during mitophagy might involve the investigation of Gyp1 localization at mitochondria by using the Delta Vision microscope.

The role of Gyp1 in the Cvt-pathway was formerly reported (Kramer et al., 2017) and could be confirmed in this study. Gyp1 was additionally identified as a possible regulator of mitophagy. An AIM-dependent role of Gyp1 was observed in mitophagy, but not for the Cvt-pathway. The GAP activity of Gyp1 seems to be needed for both pathways. The Ypt1-Q67L mutant that is restricted to the GTPbound form is also reported to be defective in pApe1 maturation (Lynch-Day et al., 2010). This supports the presumed role of Gyp1 in the Cvt-pathway. Investigation of other selective pathways like pexophagy might be useful to further define the role of Gyp1 in more detail.

\subsection{Gyp1 and Atg8 co-localize at the PAS}

GFP-Atg8 co-localizes with a sub-fraction of Cherry-Gyp1 dots in fluorescence microscopy (Fig. 4.7A and B). Atg8 is known to localize at the PAS and is commonly used as PAS marker (Delorme-Axford, 2016; Suzuki and Ohsumi, 2010). Gyp1 is reported to localize to the Golgi. Co-localization studies were performed with Sec7 (Sclafani et al., 2010) and Bet3 (Du and Novick, 2001). Bet3 is a core subunit of the TRAPP complexes (Sacher et al., 2000), which are reported to play important roles at the early and late Golgi and in autophagy (Kim et al., 2016). Besides the fact that Gyp1 co-localized with Bet3 only partially, this study supports a potential role of Gyp1 at the PAS, where also the TRAPPIII complex contributes to PAS formation (Noda et al., 2013; Lynch-Day et al., 2010). This result was confirmed by other studies. Kramer et al. (2017) co-localized the 
PAS marker Ape1 with Gyp1 under nutrient-rich conditions as well as after rapamycin treatment. Lynch-Day et al. (2010) showed that its GEF Trs85 targets Ypt1 to the PAS, and the requirement of Gyp1 as the GAP of Ypt1 also in this context is likely. Thus, the localization of Gyp1 at the PAS has been reported formerly, but not by co-localization with Atg8. The co-localization of both proteins at the PAS furthermore supports their predicted interaction and the role of Gyp1 in autophagy.

The co-localization of Gyp1 and Atg8 does not depend on their interaction (Fig. 4.7A and $B$ ), indicating that both proteins are recruited independently to the PAS. Mutating the R343 residue (R343K) in Gyp1 has also no significant effect on the co-localization rate of both proteins, indicating that the GAP activity of Gyp1 is also not needed for Atg8 and Gyp1 co-localization at the PAS (Fig. 4.7A and B). Cherry-Gyp1 was expressed under the MET25 promoter, which is repressed in the presence of methionine. Thus, Cherry-GYP1 was expressed in the presence of $0.3 \mathrm{mM}$ methionine to induce nearly endogenous protein levels. Overexpression of proteins or the Cherry tag might lead to their mislocalization in the cell and can cause side-effects, which should be considered. The functionality of the plasmid expressing Cherry-Gyp1 was therefore examined by measurement of the pApe1 maturation rate. Wild type levels of mature Ape1 indicate that the plasmid fulfills its function (Fig. 4.7C and D). However, the Delta Vision microscope might be more sensitive and the pApe1 maturation assay does not guarantee that Cherry-Gyp1 localization is completely wild type-like. Nevertheless, one could assume that the MET25 promoter affects all Gyp1 constructs similarly and that differences in their localization due to the mutations should be still observable. The Cherry tag of the protein might also cause similar effects. This could be tested in further studies. As Atg8 is commonly used as a PAS marker (Delorme-Axford et al., 2015), the co-localization of both proteins is predicted to occur at the PAS in this study. This assumption could be further investigated. If both proteins interact, an accumulation of Cherry-Gyp1 (due to the MET25 promoter) might lead to increased binding to GFP-Atg8 and thereby to accumulation of GFP-Atg8. These structures might be identified as false positive PAS. Further studies could include the expression of Gyp1 from its endogenous promoter or chromosomally tagged Gyp1 and co-localization of Atg8 with a PAS 
marker like Ape1 to exclude that Atg8 forms additional PAS-like dots upon overexpression of Gyp1. To further analyze the role of Gyp1 in autophagy, the Cvt-pathway was examined.

\subsection{The role of Gyp1 in the Cvt-pathway}

By using the Delta Vision microscope, the recruitment of Atg proteins responsible for the Cvt-pathway and PAS formation under nutrient-rich conditions were investigated. First, Atg11 assembles with the Cvt-complex by direct interaction with Atg19 (Shintani et al., 2002). Together they recruit the serine-threonine kinase Atg1 (Kamber et al., 2015), which has been reported to interact with Ypt1GTP (Lipatova et al., 2012). Formation of the PAS proceeds by recruitment of the class III PI3-kinase complex (Suzuki et al., 2015) by the Atg1 kinase complex and Atg9. The class III PI3-kinase complex is needed for the generation of PI3P (Kihara et al., 2001), which is then needed for proper formation of the phagophore. Depending on PI3P, Atg21 defines the lipidation site of Atg8 by linking Atg8 and the E3-like enzyme complex consisting of Atg12, Atg5 and Atg16 at the PAS (Juris et al., 2015). Atg8 is thereby linked to PE and localizes to the inner and outer side of the isolation membrane. On the outer surface, Atg8 is removed before fusion of the autophagosome with the vacuole (Kirisako et al., 2000).

\subsubsection{The Cvt-complex assembles properly in the absence of Gyp1}

Assembly of the Cvt-complex at the PAS was investigated by fluorescence microscopy of Atg11 and Ape1. Ape1-RFP and Cherry-Atg11 were expressed in gyp $1 \Delta$ cells and in gyp $1 \Delta$ gyp5 gyp8s cells (Fig. 4.8A and C). The PAS is detectable in yeast as one dot-like structure per cell, localized near the vacuole. Most Atg proteins localize at the PAS at least transiently. Therefore, Atg proteins are tagged with a fluorescent tag and visualized in fluorescence microscopy to investigate their proper localization to one dot-like structure close to the vacuole, representing the PAS.

The localization of both proteins to the presumed PAS is wild type-like (Fig. 4.8B and $\mathrm{D})$. After pApe1 is synthesized, it forms homodecamers and is assembled by its propeptide into dodecamers that build the Ape1 complex (Kim et al., 1997; Yamasaki and Noda, 2017). This propeptide also interacts with the coiled-coil 
domain of Atg19, the Cvt-receptor (Scott et al., 2001). Atg19 then recruits Atg11, resulting in formation of the Cvt-complex (Yorimitsu and Klionsky, 2005). As the two proteins, pApe1 and Atg11 are wild type-like localized to the PAS in this study, it could be assumed that the formation of the Cvt-complex and its assembly with the scaffold protein is not affected be deletion of GYP1. This could be confirmed by analyzing the co-localization of Atg11 and Ape1 with an additional

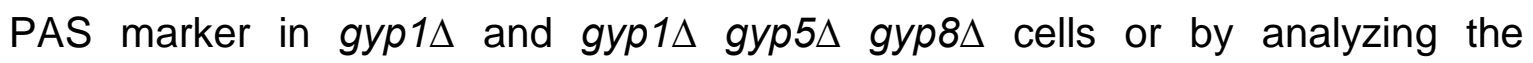
localization of Atg19 as additional marker for the Cvt-complex.

Atg11 is a downstream effector of Ypt1 and has been reported to interact with Ypt1 preferentially in its GTP-bound form. In the ypt1-1 (T40K) mutant, interaction of both proteins is disrupted, indicating that the respective residue is needed for the interaction of both proteins. This mutant leads to multiple dots of GFP-Atg11 compared to a single dot per cell in wild type. Thus, the Atg11-Ypt1 interaction is reported to be important for the localization of Atg11 to the PAS (Lynch-Day et al., 2010; Lipatova et al., 2012). Interaction of the activated Ypt1 with Atg11, leads to the recruitment of further proteins to the PAS e.g. Atg8 (Lipatova et al., 2012). In the ypt1-1 mutant, Cherry-Atg8 appears as multiple dots in the cell and the GFPAtg1 signal is diffuse (Lipatova et al., 2012). Thus, Ypt1 and its interaction with Atg11 seem to be important for the recruitment of Atg1 and Atg8 to the PAS.

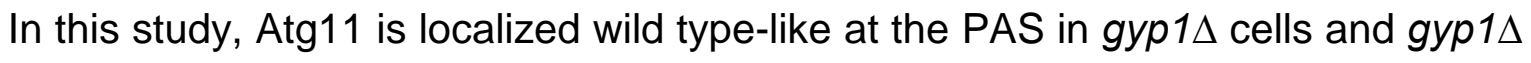
gyp5 $5 y$ g $8 \Delta$ cells. Atg11 preferentially interacts with the GTP-bound form of Ypt1 and Gyp1 induces the GTP hydrolysis on Ypt1. If Ypt1 and Atg11 interact preferentially in the GTP bound form of Ypt1, one could assume that Gyp1 might be important for the disassembly of the two proteins as it has been shown in this study for Atg1 (Chapter 4.4.2). The results of this study indicate that Gyp1induced GTP hydrolysis is not required to release Atg11 from Ypt1. Here, Ypt1 might be hydrolyzed by a different GAP. It is also possible that Atg11 and Atg1 compete for the same effector binding site on Ypt1 and that Atg1 replaces Atg11. To determine the interaction of Atg11 and Ypt1, Lipatova et al. (2012) used in vitro co-immunoprecipitation experiments and the BiFC (bimolecular fluorescence complementation) assay. For the latter, they used Atg1 as a negative control as they expected no interaction with Ypt1. In contrast to that, the interaction of Atg1 and Ypt1 was published later (Wang et al., 2013). Thus, the BiFC assay might not 
be a suitable method to determine the protein interactions at the PAS. Therefore, the interaction of Atg11 and Ypt1 might need further investigation.

The GEF of Ypt1, Trs85 seems to interact with Ypt1 at the PAS. Trs85 is reported to localize to Atg9-containing vesicles (Lipatova et al., 2012) and to be involved in the transport of Ypt1 -that also localizes on Atg9-containing vesicles- to the PAS (Kakuta et al., 2012). As Trs85 is transported to the PAS on Atg9 vesicles, one can speculate that also Gyp1 is transported on Atg9 vesicles. Du and Novick (2001) assume that Gyp1 is predominantly bound to membranes (presumably the Golgi) and also co-fractionated Gyp1 with Bet3, a TRAPP complex component. This indicates that Gyp1 might localize to the TRAPPIII complex. Although, proteomic analysis detected Gyp8 as a component of the Atg9 vesicles but not Gyp1 (Kakuta et al., 2012). Transport of Gyp1 to the PAS has not been examined in more detail in this study and could be further investigated e.g. by co-localization of Gyp1 and the TRAPPIII complex components/Atg9 vesicles using fluorescence microscopy.

\subsubsection{Gyp1 leads to efficient dissociation of the Ypt1-Atg1 complex}

Atg1 has been identified as effector of Ypt1 (Wang et al., 2013). Therefore, Atg1GFP was examined using fluorescence microscopy (Fig. 4.9A and B). In gyp $1 \Delta$ as well as in gyp1 $1 \Delta y p 5 \Delta$ gyp8 $\Delta$ cells, the number of Atg1-GFP dots/cell increased significantly compared to wild type cells, using the Delta Vision microscope (Fig. 4.9B). These results were further investigated by a GFP-TRAP approach (Fig. 4.9C and D). Here, Ypt1 co-precipitation with Atg1-GFP is increased in gyp1د cells compared to wild type cells (Fig. 4.9D).

Wang et al. (2013) formerly published the interaction of Ypt1 and Atg1. This interaction is preferred when Ypt1 is in its GTP-bound form. The interaction of Ypt1 and Atg1 is even conserved in mammals. The mammalian homolog of Atg1, ULK1 interacts with Rab1, the Ypt1 homolog (Wang et al., 2013). Analysis of Atg1-GFP via the Delta Vision microscope and the GFP-TRAP approach in this study lead to the assumption that Gyp1 acts as a GAP to induce hydrolysis of the Ypt1-bound GTP, leading to efficient disassembly of the Ypt1-Atg1 complex. Further studies on this part should include co-localization of Atg1-GFP with a PAS 
marker like Ape1 to determine if the observed dots indeed correspond to PAS structures or if they represent mislocalized Atg1-GFP.

The ypt1-2 mutant (A83E) is defective in binding to GTP and thus restricted to its inactive, GDP-bound form. In this mutant, localization of Atg1 occurs to wild type levels under nutrient-rich conditions but Atg1 mislocalizes after rapamycin treatment to multiple small dots (Wang et al., 2013). Thus, Ypt1-GTP seems to be required for Atg1 recruitment under starvation but not under nutrient-rich conditions. In this study, Atg1-GFP was analyzed only under nutrient-rich conditions, where an increase in the number of Atg1-GFP dots/cell was shown. These results, together with the observation made by Wang et al. (2013), indicate that the observed enrichment of Atg1-GFP is not caused by an enhanced recruitment of Atg1, but by a disrupted proceeding of autophagy in the following steps. The interaction of Atg1/Atg11 and Ypt1 should be further analyzed, e.g. by performing further interaction studies to identify the interaction sites of the proteins.

\subsubsection{Disassembly of the Ypt1-Atg1 complex is a prerequisite for Atg14 recruitment}

The Atg1 complex is important for early steps of autophagy and for the recruitment of Atg proteins to the PAS. To further investigate the Ypt1-Atg1 complex, Atg proteins that are recruited later to the PAS were examined. Proper localization of Atg14 to the PAS was investigated by analyzing chromosomally tagged Atg14-GFP cells in the Delta Vision microscope (Fig. 4.10A). Upon deletion of GYP1, Atg14-GFP dots/cell decrease. This effect is even stronger in gyp $1 \Delta$ gyp5 gyp8 8 cells (Fig. 4.10B).

The recruitment of Atg14 to the PAS is not fully understood and the observed defect in Atg14 recruitment in the GYP1 deletion strain might have different reasons. Atg14 might be recruited to the PAS by the HORMA domain of Atg13 (Jao et al., 2013). Enhanced recruitment of Atg14 to the PAS is observed in atg1ム cells, but impaired in atg $9 \Delta$ and atg13 $\Delta$ cells (after rapamycin treatment), indicating that Atg9 and Atg13 are needed for Atg14 recruitment under starvation conditions (Suzuki et al., 2007). Atg14 is furthermore not properly located to the 
PAS in trs85 cells (Kakuta et al., 2012). Trs85 serves as a GEF of Ypt1 (LynchDay et al., 2010). This mislocalization of Atg14 in trs85 cells was only observed under nutrient-rich conditions and absent after rapamycin treatment (Kakuta et al., 2012). This indicates that there might be a difference in Atg14 recruitment under nutrient-rich and under starvation conditions and that Ypt1 is involved in this process. Lipatova et al. (2012) assumed that interaction of Ypt1 and Trs85 occurs at Atg9 vesicles. Ypt1 regulates autophagy by interacting with its effector Atg1, which tethers Atg9 vesicles (Stanley et al., 2015). Disassembly of Atg1 and Ypt1 might be a prerequisite for proper nucleation of the phagophore due to Atg9 vesicle tethering by Atg1 (Rao et al., 2015). If the tethering of Atg9 vesicles is impaired, further recruitment of proteins like Atg14 to the PAS might be affected because Atg9 is involved in Atg14 recruitment. In a cell-free assay, Ypt1 has been shown to be important for vesicle fusion (Segev, 1991). Thus, the disassembly of the Ypt1-Atg1 complex might be needed for the proposed vesicle tethering or fusion function of Atg1 (Wang et al., 2013; Ragusa et al., 2013) or for the vesicle fusion function of Ypt1 (Segev, 1991). This might affect proper recruitment of Atg14 to the PAS.

The recruitment of Atg14 to the PAS was more closely investigated by expressing

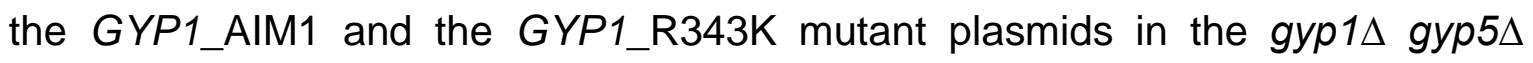
gyp8 $\Delta$ strain (Fig. 4.11A and B). The recruitment of Atg14-GFP is impaired in cells containing the Gyp1_R343K mutant and wild type-like in the Gyp1_AIM1 mutant containing cells (Fig. 4.11B). The impaired disassembly of the Ypt1-Atg1 complex might cause this mislocalization of Atg14-GFP in the Gyp1_R343K mutant. Wild type levels of Atg14-GFP in the Gyp1_AIM1 mutant indicate normal PI3P levels at the PAS and efficient recruitment of Atg8 should be possible.

\subsubsection{GAP activity of Gyp1 is needed for recruitment of Atg8 to the PAS}

The recruitment of GFP-Atg8 to the PAS was examined in gyp1 $1 \Delta$ cells and gyp1 $\Delta$ gyp5 gyp8s cells using the Delta Vision microscope (Fig. 4.12A). The recruitment of GFP-Atg8 was impaired in both strains (Fig. 4.12B). Again, the effect was stronger after deletion of all three GAPs. In Figure 4.11A, a clear mislocalization of Atg8 to the cytoplasm is visible in the gyp1 $\Delta$ strain, which is in

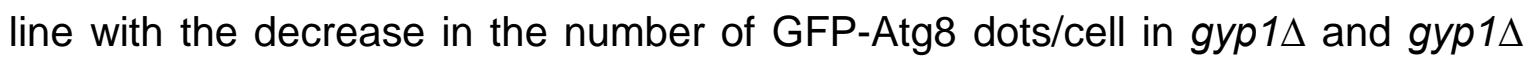


gyp5 gyp8s cells. Furthermore, a mislocalization of GFP-Atg8 upon GYP1 deletion under starvation conditions was published (Kramer et al., 2017).

The reason for the Atg8 recruitment defect was further analyzed by expressing

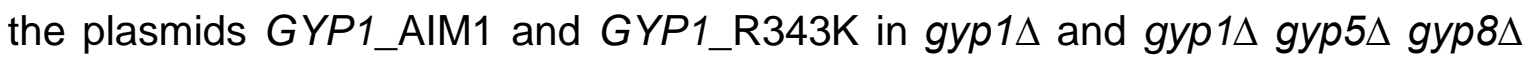
cells. In gyp $1 \Delta$ cells, containing the Gyp1_AIM1, a wild type like recruitment of Atg8 to the PAS was observed. Gyp1_R343K caused a decrease in recruitment of Atg8. Thus, Atg8 recruitment depends on the GAP activity of Gyp1 but Atg8 recruitment to the PAS is not mediated via the AIM of Gyp1. The defect in Atg8 recruitment is more likely due to the Ypt1-Atg1 disassembly defect that disrupts proper recruitment of Atg proteins to the PAS. Decreased Atg14 recruitment to the PAS in the GYP1 deletion strain supports this idea. Recruitment of Atg14 and the PI3P complex is a prerequisite for Atg8 recruitment to the PAS (Suzuki et al., 2007). Co-localization studies of Atg8 and the PAS marker Ape1 in gyp $1 \Delta$ and gyp1 gyp5 $5 y$ g $8 \Delta$ cells could further prove these data.

In gyp1s gyp5 gyp8 8 cells containing Gyp1_AIM1, Atg8 recruitment is impaired (Fig. 4.13C and D). For most experiments, the additional deletion of GYP5 and GYP8 increased the observed phenotype. For example, the Cvt-pathway is more severely impaired and Atg14-GFP and GFP-Atg8 recruitment to the PAS is more severely affected in gyp $1 \Delta$ gyp $5 \Delta$ gyp $8 \Delta$ cells. Thus, the recruitment of Atg8 might indeed depend on Gyp1, but the defect is not observable in single mutant cells. The three GAPs Gyp1, Gyp5 and Gyp8 have been reported to function as GAP for Ypt1. It is therefore likely, that Gyp5 and Gyp8 are required to disassemble the Ypt1-Atg1 complex in the absence of Gyp1.

Interestingly, the Atg1 phenotype, observed by the Delta Vision microscope (Fig. 4.9A and B), is not further increased in gyp1 $14 y p 5 \Delta$ gyp8 $\Delta$ cells compared to the gyp $1 \Delta$ cells. Thus, the proposed overlapping function of the three GAPs seems to be absent in context with the Ypt1-Atg1 complex. The further impaired recruitment of Atg14 to the PAS in gyp1s gyp5 gyp8s cells compared to gyp1s cells (Fig. 4.10) argues against this assumption and points to an overlapping function of the Gyp proteins in disassembly of the Ypt1-Atg1 complex.

In the localization of GFP-Atg8 at the PAS, expression of Cherry-GYP1_R343K leads to a decrease in the number of GFP-Atg8 to 0.14 dots/cell in gyp $1 \Delta$ cells 
and to 0.13 dots/cell in gyp1s gyp5s gyp8s cells expressing untagged GYP1_R343K (Fig. 4.12). Thus, additional deletion of GYP5 and GYP8 did not increase the effect of the Gyp1_R343K mutant. Interestingly, the effect only

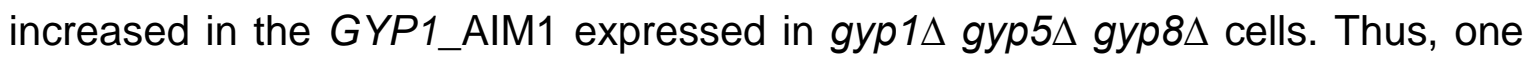
could speculate that the GAPs Gyp5 and Gyp8 might compensate the function of Gyp1 as a GAP and/or as Atg8 interaction partner. To test this hypothesis the interaction of Atg8 with Gyp5 and Gyp8 could be analyzed in a GYP1 deletion strain for example by the GFP-TRAP approach. Possibly the interaction of Atg8 and Gyp5/Gyp8 becomes detectable when Gyp1, and thus the main interaction partner of Atg8, is absent. Also a co-localization of Gyp5 and Gyp8 with a PAS marker in gyp1s cells should then be detected by using the Delta Vision microscope. As all GAPs are located differently in cell, deletion of GYP1 might lead to a partial recruitment of the proteins to the PAS to substitute the function of Gyp1.

For GFP-Atg8 recruitment experiments, two different GYP1 plasmids were used. One was tagged with Cherry, expressed form the MET25 promoter and used for

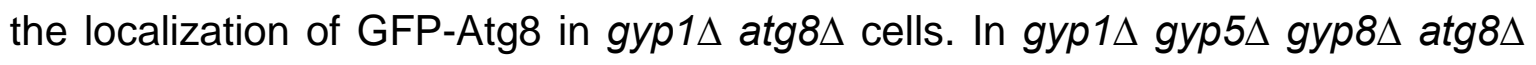
cells, GYP1 and the respective mutants were expressed untagged from their endogenous promoter (Fig. 4.13). Thus a direct comparison of the two experiments might be difficult. For example, the number of GFP-Atg8 dots/cell is equal in Gyp1_AIM1 containing cells in the two different strains. Thus, the direct comparison of the two experiments would lead to the assumption that the Gyp1_AIM1 effect on GFP-Atg8 localization is only observable due to a higher number of GFP-Atg8 dots/cell in the Gyp1_WT. This could also be an effect of the two different experimental setups. To make the two experiments better comparable, GYP1 and the respective mutants have to be expressed in gyp1 $\operatorname{atg} 8 \Delta$ cells from their endogenous promoters.

The GFP-Atg8 localization experiments lead to the suggestion that the interaction of Gyp1 and Atg8 is not important for proper Atg8 recruitment to the PAS in gyp1 cells. Proper recruitment of GFP-Atg8 is not affected in Gyp1_AIM1 containing cells but reduced in Gyp1_R343K containing cells, indicating a defect in GFPAtg8 recruitment due to improper Ypt1-Atg1 complex disassembly. The deletion of GYP1, GYP5 and GYP8 leads to a Gyp1_AIM1-dependent reduction in Atg8 
recruitment to the PAS (Fig. 4.13 A and B). The enhanced phenotype upon deletion of GYP5 and GYP8 might indicate overlapping functions and an interaction of the two proteins with Atg8. The defect in efficient disassembly of the Ypt1-Atg1 complex has an effect on Atg8 recruitment, detectable in the GYP1_R343K mutant expressing cells. Recruitment of Atg8 to the PAS could be further analyzed by co-localization of Atg8 with the PAS marker Ape1 or Atg19, the receptor for the Cvt-pathway. This would exclude that the observed GFP-Atg8 dots are not representing the PAS due to deletion of GYP1, GYP5 and GYP8.

\subsection{Gyp1 is involved in cargo recognition by Atg8}

Atg8 and Atg14 seem to be properly recruited to the PAS in the Gyp1_AIM1 mutant. This led to the assumption that later stages of phagophore formation might require the interaction of Atg8 and Gyp1. After conjugation of Atg8 to PE, Atg8-PE is transferred from the lipidation complex to the inner and outer side of the autophagosome. On the outer side, Atg8 is associated with the Atg12Atg5/Atg16 complex, which builds a coat-like structure (Kaufmann et al., 2015). Here, Atg8 is removed before the autophagosome is completed (Kirisako et al., 2000). On the inner side, Atg8 is part of the cargo complex (Yamasaki and Noda, 2017; Zaffagnini and Martens, 2016). Most of the cargo receptors in autophagy interact with Atg8 to direct their cargo to the autophagosomal membrane. During the Cvt-pathway, Atg11 targets the pApe1-Atg19 complex to the PAS (Shintani et al., 2002). At the PAS, Atg19 interacts with Atg8 in an AIM-dependent manner. Mutating the AIM in Atg19 leads to defects in the Cvt-pathway, but not in macroautophagy (Noda et al., 2008). Similar to that, Atg32 directly interacts with Atg8 via the WQAI (amino acids 86 to 89 ) sequence in its $\mathrm{N}$-terminal region to tether mitochondria to the autophagic membrane. Mutation of the Atg32-AIM leads only to partial defects in mitophagy, indicating that the AIM is not essential (Kondo-Okamoto et al., 2012). Furthermore, Atg32 interacts with Atg11, and the interaction is supposed to initiate mitophagy (Kondo-Okamoto et al., 2012). The ER-phagy receptor Atg39 binds to Atg8 during degradation of the perinuclear ER and Atg40 binds to Atg8 during degradation of cytoplasmic ER by autophagy. Both receptors interact with Atg8 in an AIM-dependent manner (Mochida et al., 2015). 
The proposed role of Gyp1 in the cargo complex with Atg8 was examined by the GFP-TRAP approach (Fig. $4.14 \mathrm{~A}$ and B). Atg32 is the specific receptor for

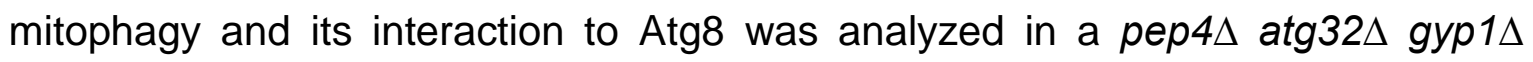
strain. Pep4 is a vacuolar aspartyl protease and its deletion should lead to the stabilization of the protein (Teichert et al., 1989). The Gyp1 mutants GYP1_AIM1 and GYP1_R343K were expressed untagged together with GFP-ATG32 and 2xFlag-ATG8. Binding of 2xFlag-Atg8 and GFP-Atg32 is significantly reduced in

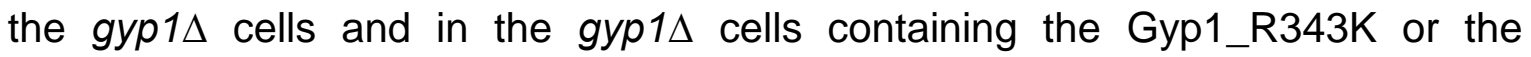
Gyp1_AIM1 mutant (Fig. 4.14B). For calculation of the 2xFlag-Atg8 binding rate to GFP-Atg32, the bound fractions of GFP-Atg32 were not considered because of the severe degradation of the protein even after PEP4 deletion, which was formerly reported (Levchenko et al., 2016). Therefore, the number of independent experiments was increased.

Thus, these experiments could be further optimized e.g. to avoid the degradation of Atg32. Nevertheless, these experiments reveal a defect in Atg8-Atg32 interaction upon GYP1 deletion (Fig. 4.14 A and B).

The role of Gyp1 in formation of the cargo complex was further investigated by using the Cvt-pathway receptor Atg19. For the split-ubiquitin assay, binding of Atg8 and Atg19 was analyzed in the gyp1s strain and compared to cells containing the Gyp1_WT. The interaction of Atg8 and Atg19 is reduced upon deletion of GYP1 (Fig. 4.15), indicating a role of Gyp1 in cargo complex formation. However, this effect might also be caused by the function of Gyp1 in disassembly of the Ypt1-Atg1 complex. To further investigate the role of Gyp1, this experiment could be repeated with the respective Gyp1 mutants impaired either in the GAP function or in binding to Atg8. A disadvantage of the split-ubiquitin assay is that the ubiquitin remains stable and keeps the proteins bound together upon interaction. Thus, small differences in binding might be difficult to detect. Furthermore, these experiments are evaluated by eye and not additionally by quantification, making a final statement of the results difficult when only small effects can be observed.

A co-immunoprecipitation experiment similar to the Atg32 experiment described above could be used to further investigate the binding of Atg8 and Atg19 in dependence on Gyp1. In addition, the receptor Atg36, important for pexophagy, 
might be analyzed for its binding to Atg8 in the absence of Gyp1. Thereby, it might be possible to elucidate if Gyp1 is needed for all types of selective autophagy or if its function is restricted to distinct sub-types.

At the outer, concave side of the phagophore, Atg8 tethers the cargo to the isolation membrane. Atg8 forms a complex with Atg12 Atg5/Atg16, building a scaffold-like structure. Atg8 links Atg12-Atg5 to the membrane by an AIMdependent interaction with Atg12. It has been shown that cargo receptors like Atg32 compete with the Atg12-Atg5 complex for interaction with Atg8-PE, thereby allowing the spatial segregation of Atg8-PE (Kaufmann et al., 2014). By using an in vitro reconstituted system it has been shown how Atg8 conjugation to PE is coupled to the presence of cargo (Fracchiolla et al., 2016). The cargo receptors Atg19 and Atg34 and also mammalian cargo receptors like p62 seem to interact with the Atg12 Atg5/Atg16 E3-like enzyme (Fracchiolla et al., 2016). The authors show that this interaction is mediated by the AIM in Atg19 and an AIM binding site in Atg5 (Fracchiolla et al., 2016). Thereby, Atg19 targets the Ape1 complex to the Atg12 Atg5/Atg16 complex. Atg8 then might replace Atg5 on Atg19 and is conjugated to PE. Gyp1 might be important for the spatial distribution of Atg8. The cargo receptors have a higher affinity for Atg8-PE than Atg12. Thus, Gyp1 might be required to support the localization of Atg8 on the convex side of the phagophore or to avoid mislocalization of the cargo on the convex side. If Atg8$\mathrm{PE}$ is not correctly targeted to the concave side of the phagophore, recruitment of the cargo and binding of Atg32/Atg19 might be reduced. This could be further investigated by comparing the Atg8 levels inside the mature autophagosome between wild type and GYP1 knockout cells. This predicted role of Gyp1 would be in line with the observation of this study that Gyp1 is only needed during selective forms of autophagy. Contrary to this, Kramer et al. (2017) postulate a function also during non-selective autophagy.

Taken together, these results lead to the assumption that Gyp1 might have a second function besides its GAP activity.

\subsection{Gyp1 has a dual function during selective autophagy}

The results of this study indicate that Gyp1 has two functions during autophagosome formation (Fig. 5.1). First, it acts as a GAP on Ypt1 and leads to 
efficient disassembly of the Ypt1-Atg1 complex. This is a prerequisite for further recruitment of Atg proteins, as shown for Atg14. Atg14 then directs the PI3P kinase complex to the PAS. Depending on PI3P, Atg21 organizes proper conjugation of Atg8 to PE. Then Atg8 is transferred to the outer complex with Atg12-Atg5/Atg16, where it contributes to the formation of a coat-like structure. On the inner side, Atg8 interacts with the cargo receptors. Here, Gyp1 is supposed to fulfill its second function in directing Atg8 to the inner side of the autophagosome, indicated by a reduced interaction of Atg8 and Atg32 upon GYP1 deletion.

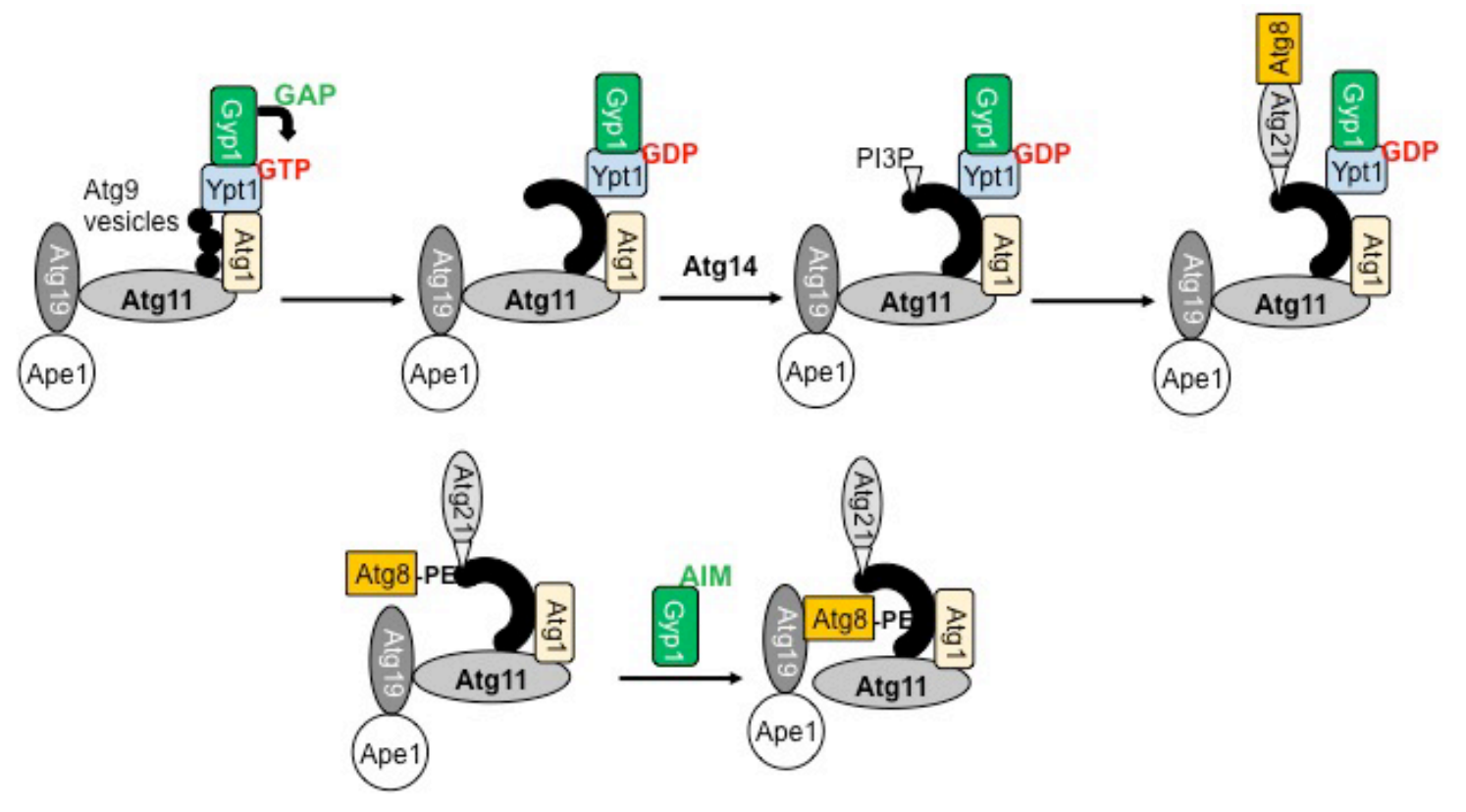

\section{Figure 5.1: Gyp1 has dual functions in autophagy}

During early steps of autophagy, Gyp1 functions as a GAP for Ypt1. It hydrolyzes the Ypt1-bound GTP, which is a prerequisite for the efficient disassembly of the Ypt1-Atg1 complex and for the recruitment of downstream Atg proteins like Atg14 to the PAS. Atg14 directs the PI3P kinase complex to the PAS. Atg21 then organizes, depending on PI3P, proper conjugation of Atg8 to PE. Atg8 is then transferred to the outer side of the phagophore, where it contributes to the formation of the Atg12-Atg5/Atg16 coat-like structure. On the inner side, Atg8 interacts with the selective cargo receptors like Atg19 and Atg32. Gyp1 might fulfill its second AIM-dependent function in facilitating the transfer of Atg8 from the lipidation complex or in facilitating the interaction between Atg8 and the cargo receptors.

The molecular mechanism by which Gyp1 directs Atg8 or supports its interaction with the cargo receptor remains elusive, but the obtained data considerably narrowed down its function. 
Additional functions have also been shown also for other GAP proteins, besides the regulation of Ypt/Rabs, their interaction with Atg8 homolouges has been reported. A screen for Rab GAPs, which co-localize with LC3-positive membranes and interact with Atg8 modifiers, identified 14 Rab GAPs (Popovic et a., 2012). A study from Longatti et al. (2012) screened for GAP proteins and identified 11 putative regulators of starvation-induced autophagy. They identified the GAP TBC1D14 as a negative regulator of Rab1. TBC1D14 interacts with the human Atg1 homolog ULK1 and partially co-localizes with LC3. It furthermore regulates Atg9 trafficking and interacts with the TRAPPIII-like complex, which acts as a GEF for Rab1 and recruits it to the membrane (Lamb et al., 2016). Thus, GAP proteins can have additional functions besides the regulation of Ypt/Rabs can interact with Atg8 homologs. These data support the results of this study that proposes an additional function of Gyp1 besides its GAP activity.

Several observations during this and other studies make it difficult to clearly define the role of Gyp1. There are overlapping functions of Gyp1 with other GAPs (De Antoni et al., 2002; Du and Novick, 2001) and GAPs are reported to have other functions besides their GAP activity (Longatti et al., 2012; Lamb et al., 2015). Furthermore, different functions during autophagy are also reported for Atg8 (Shpilka et al., 2011). Thus, the reduced levels in receptor binding to Atg8 can have different reasons: Gyp1 might be important for the interaction of Atg8 with the receptor or needed for sorting of Atg8 to the different complexes at the phagophore.

Gyp1 could also avoid premature cleavage of Atg8-PE. Atg4 has two functions in Atg8 processing. First, it cleaves the C-terminal arginine of Atg8 to allow its conjugation to PE. Later, it deconjugates Atg8 from PE at the outer side of the completed phagophore. Different mechanisms are under debate how Atg4 is regulated. For example, Atg4 preferentially interacts to Atg8-PE in an AIMdependent manner or Atg4 is phosphorylated by Atg1 (Skytte Rasmussen et al., 2017; Sánchez-Wandelmer et al., 2017). Gyp1 binds to the AIM binding site in Atg8 and thereby possibly prevents binding of Atg4 and premature cleavage of Atg8 from PE. Premature cleavage of Atg8-PE would lower the amount of Atg8 at the phagophore and cause a decreased ability of Atg8 to bind to the cargo receptors Atg32 and Atg19. Atg4 could also occupy the AIM binding site of Atg8 
that would be not accessible for cargo binding. But, the number of GFP-Atg8

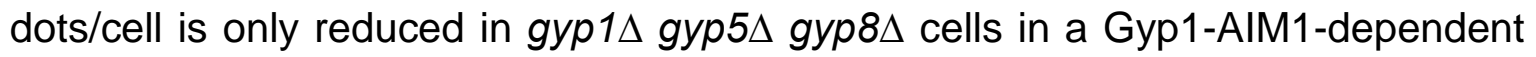
manner (Fig. 4.13D). This could indicate premature cleavage of Atg8 from PE. Contrary to this assumption, this effect is not observable in the GYP1_AIM1 mutant expressed in gyp1 $\Delta$ cells (Fig. 4.13B) but again, the two GAPs Gyp5 and Gyp8 might compensate the function of Gyp1 and the effect might be not

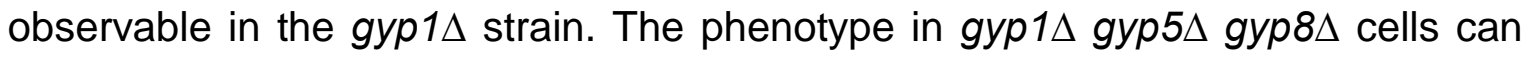
be furthermore explained by an initial defect in Atg8 recruitment to the PAS as the number of GFP-Atg8 dots/cell is reduced in this strain (expressing GYP1_AIM1 and GYP1_R343K). This needs further investigation.

Recently, it has been proposed that the Ypt1 GEF, TRAPPIII, and Gyp1 regulate the Ypt1 level at the Golgi (Thomas et al., 2017). Gyp1 also plays a role in compartment identity at the Golgi (Rivera-Molina and Novick, 2009). Furthermore, the gyp1 $1 \Delta$ strain has a defect in CPY secretion (Du and Novick, 2001), indicating a defect in protein transport from the Golgi to the vacuole. The localization of Gyp1 at the PAS, proper recruitment of Atg11 and its direct AIM-dependent interaction with Atg8 clearly argue against an indirect effect of Gyp1 function at the Golgi or in ER-Golgi transport on autophagy.

\subsection{Conclusion}

This study predicts a dual role of Gyp1 during autophagy. First, Gyp1 leads to efficient dissociation of the Ypt1-Atg1 complex, necessary for further recruitment of proteins to the PAS. In later steps of autophagy, Gyp1 promotes the formation of the Atg8-cargo complex. The precise role of Gyp1 in this context remains elusive and needs further investigations. 


\section{Bibliography}

Abreu, S., Kriegenburg, F., Gómez-Sánchez, R., Mari, M., Sánchez-

Wandelmer, J., Skytte Rasmussen, M., Soares Guimarães, R., Zens, B., Schuschnig, M., Hardenberg, R., Peter, M., Johansen, T., Kraft, C., Martens, S., Reggiori, F. (2017). Conserved Atg8 recognition sites mediate Atg4 association with autophagosomal membranes and Atg8 deconjugation. EMBO Reports, 18(5):765-780

Albert, S., Will, E., Gallwitz, D. (1999). Identification of the catalytic domains and their functionally critical arginine residues of two yeast GTPase-activating proteins specific for Ypt/Rab transport GTPases. The EMBO Journal, 18(19): 5216-5225

Alemu, E. A., Lamark, T., Torgersen, K. M., Birgisdottir, A. B., Larsen, K. B., Jain, A., Olsvik, H., Øvervatn, A., Kirkin, V., Johansen, T. (2012). Atg8 family proteins act as scaffolds for assembly of the ULK complex. Journal of Biological Chemistry, 287(47):39275-39290

Alvarez, C., Garcia-Mata, R., Brandon, E., Sztul, E. (2003). COPI Recruitment is modulated by a Rab1b-dependent mechanism. Molecular Biology of the Cell, 14(5):2116-2127

Aoki, Y., Kanki, T., Hirota, Y., Kurihara, T., Saigusa, T., Uchiumi, T., Kang, D. (2011). Phosphorylation of serine 114 on Atg32 mediates mitophagy. Molecular Biology of the Cell, 22(17):3206-3217

Araki, Y., Ku, W.-C., Akioka, M., May, A. I., Hayashi, Y., Arisaka, F., Ishihama, Y., Ohsumi, Y. (2013). Atg38 is required for autophagy-specific phosphatidylinositol 3-kinase complex integrity. The Journal of Cell Biology, 203(2):299-313

Baba, M., Osumi, M., Scott, S. V., Klionsky, D. J., Ohsumi, Y. (1997). Two distinct pathways for targeting proteins from the cytoplasm to the vacuole/lysosome. Journal of Cell Biology, 139(7):1687-1695

Babst, M. (2011). MVB vesicle formation: ESCRT-dependent, ESCRTindependent and everything in between. Current Opinion in Cell Biology, 23(4):452-457

Backues, S. K., Orban, D. P., Bernard, A., Singh, K., Cao, Y., Klionsky, D. J. (2015). Atg23 and Atg27 act at the early stages of Atg9 trafficking in S. cerevisiae. Traffic, 16(2):172-190

Barbet, N. C., Schneider, U., Helliwell, S. B., Stansfield, I., Tuite, M. F., Hall, M. N. (1996). TOR controls translation initiation and early G1 progression in yeast. Molecular Biology of the Cell, 7(1):25-42 
Barrowman, J., Bhandari D., Reinisch K., Ferro-Novick S. (2010). TRAPP complexes in membrane traffic: Convergence through a common Rab. Nature Reviews Molecular Cell Biology, 11(11): 759-763

Bhandari, D., Zhang, J., Menon, S., Lord, C., Chen, S., Helm, J. R., Thorsen, K., Corbett, K. D., Hay, J. C., Ferro-Novick, S. (2013). Sit4p/PP6 regulates ER-to-Golgi traffic by controlling the dephosphorylation of COPII coat subunits. Molecular Biology of the Cell, 24(17):2727-2738

Belhedi, N., Bena, F., Mrabet, A., Guipponi, M., Souissi, C. B., Mrabet, H. K., Elgaaied, A. B., Malafosse, A., Salzmann, A. (2013). A new locus on chromosome 22q13.31 linked to recessive genetic epilepsy with febrile seizures plus (GEFS+) in a Tunisian consanguineous family. BMC Genetics, 14, 93 http://doi.org/10.1186/1471-2156-14-93

Billant, O., Blondel, M., Voisset, C. (2017). p53, p63 and p73 in the wonderland of S. cerevisiae. Oncotarget, doi: 10.18632/oncotarget.18506.

Birgisdottir, Å. B., Lamark, T., Johansen, T. (2013). The LIR motif - crucial for selective autophagy. Journal of Cell Science, 126(15):3237-3247

Bonangelino, C. J., Chavez, E. M., \& Bonifacino, J. S. (2002). Genomic screen for vacuolar protein sorting genes in Saccharomyces cerevisiae. Molecular Biology of the Cell, 13(7):2486-2501

Bos, J. L., Rehmann, H., Wittinghofer. (2007). GEFs and GAPs: critical elements in the control of small G proteins. Cell, 129(5):865-877

Bowers, K., Stevens, T. H. (2005). Protein transport from the late Golgi to the vacuole in the yeast Saccharomyces cerevisiae. Biochimica et Biophysica Acta, 1744(3):438-454

Burri, L., Lithgow, T. (2004). A complete set of SNAREs in yeast. Traffic, $5(1): 45-52$

Cai, H., Yu, S., Menon, S., Cai, Y., Lazarova, D., Fu, C., Reinisch, K., Hay, J. C., Ferro-Novick, S. (2007). TRAPPI tethers COPII vesicles by binding the coat subunit Sec23. Nature, 445:941-944

Cao, X., Barlowe, C. (2000) Asymmetric requirements for a Rab GTPase and SNARE proteins in fusion of COPII vesicles with acceptor membranes. The Journal of Cell Biology, 149(1):55-66

Cao, Y., Cheong, H., Song, H., Klionsky, D. J. (2008). In vivo reconstitution of autophagy in Saccharomyces cerevisiae. The Journal of Cell Biology, 182(4):703-713

Chan, S. N., Tang, B. L. (2013). Location and membrane sources for autophagosome formation - from ER-mitochondria contact sites to Golgiendosome-derived carriers. Molecular Membrane Biology, 30(8):394-402 
Chang, C. - Y., Huang, W. - P. (2007). Atg19 mediates a dual interaction cargo sorting mechanism in selective autophagy. Molecular Biology of the Cell,18(3):919-929

Cheong, H., Nair, U., Geng, J., Klionsky, D. J. (2007). The Atg1 kinase complex is involved in the regulation of protein recruitment to initiate sequestering vesicle formation for nonspecific autophagy in Saccharomyces cerevisiae. Molecular Biology of the Cell, 19(2):668-681

Chiang, H., Terlecky, S., Plant, C., Dice, J. F. (1989). A role for a 70-kilodalton heat shock protein in lysosomal degradation of intracellular proteins. Science, 246(4928):382-385.

Choi, A. M. K., Ryter, S. W., Levine, B. (2013). Autophagy in human health and disease. The New England Journal of Medicine, 368(7):651-662

Davies, C. W., Stjepanovic, G., Hurley, J. H. (2015). How the Atg1 complex assembles to initiate autophagy. Autophagy, 11(1):185-186

De Antoni, A., Schmitzová J, Trepte, H. H., Gallwitz, D., Albert, S. (2002). Significance of GTP hydrolysis in Ypt1p-regulated endoplasmic reticulum to golgi transport revealed by the analysis of two novel Ypt1-GAPs. The Journal of Biological Chemistry, 277(43):41023-41031

Delorme-Axford, E., Guimaraes, R. S., Reggiori, F., Klionsky, D. J. (2015). The yeast Saccharomyces cerevisiae: an overview of methods to study autophagy progression. Methods, 75:3-12

Dice, J. F. (1990). Peptide sequences that target cytosolic proteins for lysosomal proteolysis. Trends in Biochemical Science,15(8):305-309

Dinkel, H., Van Roey, K., Michael, S., Davey, N. E., Weatheritt, R. J., Born, D., Speck, T., Krüger, D., Grebnev, G., Kuban, M., Strumillo, M., Uyar, B., Budd, A., Altenberg, B., Seiler, M., Chemes, L. B., Glavina, J., Sánchez, I. E., Diella, F., Gibson, T. J. (2014). The eukaryotic linear motif resource ELM: 10 years and counting. Nucleic Acids Research, 42(Database issue), D259-D266. http://doi.org/10.1093/nar/gkt1047

Du, L. L., Collins, R. N., Novick, P. J. (1998). Identification of a Sec4p GTPase activating protein (GAP) as a novel member of a Rab GAP family. Journal of Biological Chemistry, 273(6):3253-3256

Du, L. L. Novick, P. (2001). Yeast Rab GTPase-activating protein Gyp1 localizes to the golgi apparatus and is a negative regulator of Ypt1p. Molecular Biology of the Cell, 12(5):1215-1226

Duden, R. (2003). ER-to-Golgi transport: COPI and COPII function (Review). Molecular Membrane Biology, 20(3):197-207

Dunn Jr., W. A. (1990). Studies on the mechanisms of autophagy: formation of the autophagic vacuole. Journal of Cell Biology, 110(6):1923-1933. 
Diuna, A. A., Miller, M. E., Keeney, J. B. (2014). Budding yeast for budding geneticists: a primer on the Saccharomyces cerevisiae model system. Genetics, 197(1):33-48

Farré, J. C., Burkenroad, A., Burnett, S. F., Subramani, S. (2013). Phosphorylation of mitophagy and pexophagy receptors coordinates their interaction with Atg8 and Atg11. EMBO Reports, 14(5):441-449

Farré, J. C., Subramani, S. (2016). Mechanistic insights into selective autophagy pathways: lessons from yeast. Nature Reviews Molecular Cell Biology, 17(9):537-552

Feyder, S., De Craene, J.-O., Bär, S., Bertazzi, D. L., Friant, S. (2015). Membrane trafficking in the yeast Saccharomyces cerevisiae model. International Journal of Molecular Sciences, 16(1):1509-1525

Fîlfan, M., Sandu, R. E., Zăvăleanu, A. D., GreşiŢă, A., Glăvan, D. G., Olaru, D. G., Popa-Wagner, A. (2017). Autophagy in aging and disease. Romanian Journal of Morphology and Embryology, 58(1):27-31

Fracchiolla, D., Sawa-Makarska, J., Zens, B., de Ruiter, A., Zaffagnini, G., Brezovich, A., Romanov, J., Runggatscher, K., Kraft, C., Zagrovic, B., Martens, S. (2016). Mechanism of cargo-directed Atg8 conjugation during selective autophagy. eLife, 5, e18544. http://doi.org/10.7554/eLife.18544

Geng, J., Baba, M., Nair, U., Klionsky, D. J. (2008). Quantitative analysis of autophagy-related protein stoichiometry by fluorescence microscopy. Journal of Cell Biology, 182(1):129-140

Giaever, G., Chu, A. M., Ni, L., Connelly, C., Riles, L., Véronneau, S., Dow, S., Lucau-Danila, A., Anderson, K., André, B., Arkin, A. P., Astromoff, et al. (2009). Functional profiling of the Saccharomyces cerevisiae genome. Nature, 418(6896):387-391

Gillingham, A. K., Sinka, R., Torres, I. L., Lilley, K. S., Munro, S. (2014). Toward a comprehensive map of the effectors of rab GTPases. Develomental Cell, 31(3):358-373

Goffeau, A., Barrell, B., Bussey, H., Davis, R. W., Dujon, B., Feldmann, H., Galibert, F., Hoheisel, J., Jacq, C., Johnston, M., et al. (1996). Life with 6000 genes. Science, 274(5287):546-567

Graef, M., Friedman, J. R., Graham, C., Babu, M.. Nunnari, J. (2013). ER exit sites are physical and functional core autophagosome biogenesis components. Molecular Biology of the Cell, 24(18)2918-2931

Hanada, T. Noda, N. N., Satomi, Y. Ichimura, Y., Fujioka, Y., Takao, T., Inagaki, F., Ohsumi, Y. (2007). The Atg12-Atg5 conjugate has a novel E3-like activity for protein lipidation in autophagy. Journal of Biological Chemistry, 282(52):37298-37302 
Hanahan, D. (1983). Studies on transformation of Escherichia coli with plasmids. Journal of Molecular Biology, 166(4):557-580

Haubruck, H., Prange, R., Vorgias, C., Gallwitz, D. (1989). The ras-related mouse ypt1 protein can functionally replace the YPT1 gene product in yeast. The EMBO Journal, 8(5):1427-1432

Hayashi-Nishino, M., Fujita, N., Noda, T., Yamaguchi, A., Yoshimori, T., Yamamoto, A. A subdomain of the endoplasmic reticulum forms a cradle for autophagosome formation. Nature Cell Biology, 11(12):1433-1437

He, C., Song, H., Yorimitsu, T., Monastyrska, I., Yen, W.-L., Legakis, J. E., Klionsky, D. J. (2006). Recruitment of Atg9 to the preautophagosomal structure by Atg11 is essential for selective autophagy in budding yeast. Journal of Cell Biology, 175(6):925-935

Hutagalung, A. H., Novick, P. J. (2011). Role of Rab GTPases in membrane traffic and cell physiology. Physiological Reviews, 91(1):119-149

Ichimura, Y., Imamura, Y., Emoto, K., Umeda, M., Noda, T., Ohsumi, Y. (2004). In vivo and in vitro reconstitution of Atg8 conjugation essential for autophagy. Journal of Biological Chemistry, 279(39):40584-40592

Itoh, T., Kanno, E., Uemura, T., Waguri, S., \& Fukuda, M. (2011). OATL1, a novel autophagosome-resident Rab33B-GAP, regulates autophagosomal maturation. The Journal of Cell Biology, 192(5):839-853

Janke, C., Magiera, M., Rathfelder, N., Taxis, C., Reber, S., Maekawa, H., Moreno-Borchart, A., Doenges, G., Schwob, E., Schiebel, E. (2004). A versatile toolbox for PCR-based tagging of yeast genes: new fluorescent proteins, more markers and promoter substitution cassettes. Yeast, 21(11):947-962

Jao, C. C., Ragusa, M. J., Stanley, R. E., Hurley, J. H. (2013). A HORMA domain in Atg13 mediates PI3-kinase recruitment in autophagy. Proceedings of the National Academy of Science USA, 110(14):5486-5491

Jedd, G., Richardson, C., Litt, R., Segev, N. (1995). The Ypt1 GTPase is essential for the first two steps of the yeast secretory pathway. Journal of Cell Biology, 131(3):583-590

Jiang, P., Mizushima, N. (2014). Autophagy and human dieases. Cell Research, 24:69-79

Juris, L., Montino, M., Rube, P., Schlotterhose, P., Thumm, M., Krick, R. (2015). PI3P binding by Atg21 organizes Atg8 lipidation. The EMBO Journal, 34(7):955-973

Kakuta, S., Yamaguchi, Suzuki, C., Sasaki, M., Kazuno, S., Uchiyama, Y. (2017). Small GTPase Rab1B is associated with ATG9A vesicles and 
regulates autophagosome formation. The Official Journal of the Federation of American Societies for Experimental Biology, 31(9):3757-3773

Kakuta, S., Yamamoto, H., Negishi, L., Kondo-Kakuta, C., Hayashi, N., Ohsumi, Y. (2012). Atg9 vesicles recruit vesicle-tethering proteins Trs85 and Ypt1 to the autophagosome formation site. The Journal of Biological Chemistry, 287(53):44261-44269

Kalvari, I., Tsompanis, S., Mulakkal, N. C., Osgood, R., Johansen, T., Nezis. I. P., Promponas, V. J. (2014). iLIR: a web resource for prediction of Atg8family interacting proteins. Autophagy, 10(5):913-925

Kamada, Y., Yoshino, K., Kondo, C., Kawamata, T., Oshiro, N., Yonezawa, K., Ohsumi, Y. (2010). Tor directly controls the Atg1 kinase complex to regulate autophagy. Molecular and Cellular Biology, 30(4):1049-1058

Kamber, R. A. Shoemaker, C. J., Denic, V. (2015). Receptor-bound targets of selective autophagy use a scaffold protein to activate the Atg1 kinase. Molecular Cell, 59(3):372-381

Kanki, T., Furukawa, K., Yamashita, S. (2015). Mitophagy in yeast: molecular mechanisms and physiological role. Molecular Cell Research, 1853(10):2756-2765

Kanki, T., Wang, K., Cao, Y., Baba, M., Klionsky, D. J. (2009). Atg32 is a mitochondrial protein that confers selectivity during mitophagy. Developmental Cell, 17(1):98-109

Katzmann, D. J., Babst, M., Emr, S. D. (2001). Ubiquitin-dependent sorting into the multivesicular body pathway requires the function of a conserved endosomal protein sorting complex, ESCRT-I. Cell, 106(2):145-155

Kaufmann, A., Beier, V., Franquelim, H. G., Wollert, T. (2014). Molecular mechanism of autophagic membrane-scaffold assembly and disassembly. Cell, 156(3):469-481

Kaufmann, A., Wollert, T. (2014). Scaffolding the expansion of autophagosomes. Autophagy, 10(7):1343-1345

Kaushik, S., Cuervo, A. M. (2009). Methods to monitor chaperone-mediated autophagy. Methods in Enzymology, 452:297-324

Kern, A., Dikic, I. Behl, C. (2015). The integration of autophagy and cellular trafficking pathways via RAB GAPs. Autophagy, 11(12): 2393-2397

Knop, M., Siegers, K., Pereira, G., Zachariae, W., Winsor, B., Nasmyth, K., Schiebel, E. (1999). Epitope tagging of yeast genes using a PCR-based strategy: more tags and improved practical routines. Yeast, 15:963-972

Kihara, A., Noda, T., Ishihara, N., Ohsumi, Y. (2001). Two distinct Vps34 phosphatidylinositol 3-kinase complexes function in autophagy and 
carboxypeptidase $\mathrm{Y}$ sorting in Saccharomyces cerevisiae. The Journal of Cell Biology, 152(3):519-530

Kim, J. J., Lipatova, Z., Segev, N. (2016). TRAPP complexes in secretion and autophagy. Frontiers in Cell and Developmental Biology, 4:20 doi: 10.3389/fcell.2016.00020

Kim, J., Scott, S. V., Oda, M. N., Klionsky, D. J. (1997). Transport of a large oligomeric protein by the cytoplasm to vacuole protein targeting pathway. The Journal of Cell Biology, 137(3):609-618

Kirisako, T., Ichimura, Y., Okada, H., Kabeya, Y., Mizushima, N., Yoshimori, T., Ohsumi, M., Takao, T., Noda, T., Ohsumi, Y. (2000). The reversible modification regulates the membrane-binding state of Apg8/Aut7 essential for autophagy and the cytoplasm to vacuole targeting pathway. The Journal of Cell Biology, 151(2):263-276

Krick, R., Bremer, S., Welter, E., Schlotterhose, P., Muehe, Y., Eskelinen, E.L., Thumm, M. (2010). Cdc48/p97 and Shp1/p47 regulate autophagosome biogenesis in concert with ubiquitin-like Atg8. The Journal of Cell Biology, 190(6), 965-973

Krick, R., Muehe, Y., Prick, T., Bremer, S., Schlotterhose, P., Eskelinen, E.-L., Millen, J., Goldfarb, D. S., Thumm, M. (2008). Piecemeal microautophagy of the nucleus requires the core macroautophagy genes. Molecular Biology of the Cell, 19(10):4492-4505

Kirisako, T., Baba, M., Ishihara, N., Miyazawa, K., Ohsumi, M., Yoshimori, T., Noda, T., Ohsumi, Y. (1999). Formation process of autophagosomes is traced with Apg8/Aut7 in yeast. The Journal of Cell Biology, 147(2):435446

Klionsky, D. J., Cueva, R., Yaver, D. S. (1992). Aminopeptidase I of Saccharomyces cerevisiae is localized to the vacuole independent of the secretory pathway. The Journal of Cell Biology, 119(2):287-299

Klionsky, D. J., Cregg, J. M., Dunn, W. A. Jr, Emr, S. D., Sakai, Y., Sandoval, I. V., Sibirny, A., Subramani, S., Thumm, M., Veenhuis, M., Ohsumi, Y. (2003). A unified nomenclature for yeast autophagy-related genes. Developmental Cell, 5(4):539-545

Kondo-Okamoto, N., Noda, N. N., Suzuki, S. W., Nakatogawa, H., Takahashi, I., Matsunami, M., Hashimoto, A., Inagaki, F., Ohsumi, Y., Okamoto, K. (2012). Autophagy-related protein 32 acts as autophagic degron and directly initiates mitophagy. The Journal of Biological Chemistry, 287(13):10631-10638

Kraft, C., Kijanska, M., Kalie, E., Siergiejuk, E., Lee, S. S., Semplicio, G., Stoffel, I., Brezovich, A., Verma, M., Hansmann, I., Ammerer, G., Hofmann, K., Tooze, S., Peter, M. (2012). Binding of the Atg1/ULK1 
kinase to the ubiquitin-like protein Atg8 regulates autophagy. The EMBO Journal, 31(18):3691-3703

Kramer, M. H., Farré, J. C., Mitra, K. Yu, M. K., Ono, K., Demchak, B., Licon, K., Flagg, M., Balakrishnan, R., Cherry, J. M., Subramani, S., Ideker, T. (2017). Active interaction mapping reveals the hierarchical organization of autophagy. Molecular Cell, 65(4):761-774

Lafourcade, C., Galan, J-M., Gloor, Y., Haguenauer-Tsapis, R., Peter, M. (2004). The GTPase-activating enzyme Gyp1p is required for recycling of interanalized membrane material by inactivation of the Rab/Ypt GTPase Ypt1p. Molecular and Cellular Biology, 24(9):3815-3826

Lamb, C. A., Nühlen, S., Judith, D. Frith, D., Snijders, A. P., Behrends, S., Tooze, S. A. (2016). TBC1D14 regulates autophagy via the TRAPP complex and ATG9 traffic. The EMBO Journal, 35(1):281-301

Lamb, C. A., Yoshimori, T., Tooze, S. A. (2013). The autophagosome: origins unknown, biogenesis complex. Nature Reviews Molecular Cell Biology, 14(12):759-774

Lee, M. C., Miller, E. A., Goldberg, J., Orci, L., Schekman, R. (2004). Bidirectional protein transport between the ER and Golgi. Annual Review of Cell and Developmental Biology, 20:87-123

Leung, K. F., Baron, R., Seabra, M. C. (2006). Thematic review series: lipid posttranslational modifications. Geranylgeranylation of Rab GTPases. The Journal of Lipid Research, 47(3):467-475

Levchenko, M., Lorenzi, I., Dudek, J. (2016). The degradation pathway of the mitophagy receptor Atg32 is re-routed by a posttranslational modification. PLOS ONE, 11(12), e0168518. http://doi.org/10.1371/journal.pone.0168518

Li, W. W., Li, J., Bao, J. K. (2012). Microautophagy: lesser-known self-eating. Cellular and Molecular Life Sciences, 69(7):1125-1136

Li, P. A., Hou, X., Hao, S. (2017). Mitochondrial biogenesis in neurodegeneration. Journal of Neuroscience Research, 95(10):2025-2029

Lipatova, Z., Belogortseva, N., Zhang, X. O., Kim, J., Taussig, D., Segev, N. (2012). Regulation of selective autophagy onset by a Ypt/Rab GTPase module. Proceedings of the National Academy of Science, 109(18): 69816986

Lipatova, Z., Hain, A. U., Nazarko, V. Y., Segev, N. (2015). Critical reviews in biochemistry and molecular biology: Ypt/Rab GTPases: principles learned from yeast. Critical Reviews in Biochemistry and Molecular Biology, 50(3):203-211 
Lipatova, Z., Majumdar, U., Segev, N. (2016). Trs33-containing TRAPP IV: a novel autophagy-specific Ypt1 GEF. Genetics, 204(3):1117-1128

Lipatova, Z., Segev, N. (2012). A Ypt/Rab GTPase module makes a PAS. Autophagy, 8(8):1271-1272

Longatti, A., Lamb, C. A., Razi, M., Yoshimura, S., Barr, F. A., Tooze, S. A. (2012). TBC1D14 regulates autophagosome formation via Rab11- and ULK1-positive recycling endosomes. Journal of Cellular Biology, 197(5):659-675

Longtine, M. S., McKenzie, A. 3rd, Demarini, D. J., Shah, N. G., Wach, A., Brachat, A., Phillipsen, P., Pringle, J. R. (1998). Additional modules for versatile and economical PCR-based gene deletion and modification in Saccharomyces cerevisiae. Yeast, 14(10):953-961

Loewith, R., Hall, M. N. (2011). Target of rapamycin (TOR) in nutrient signaling and growth control. Genetics, 189(4):1177-1201

Lord, C., Bhandari, D., Menon, S., Ghassemian, M., Nycz, D., Hay, J., Ghosh, P., Ferro-Novick, S. (2011). Sequential interactions with Sec23 control the direction of vesicle traffic. Nature, 473(7346):181-186

Lynch-Day, M. A., Bhandari, D., Menon, S., Huang, J., Cai, H., Bartholomew, C. R., Brumell, J. H., Ferro-Novick, S., Klionsky. D. J. (2010). Trs85 directs a Ypt1 GEF, TRAPPIII, to the phagophore to promote autophagy. Proceedings of the National Academy of Science, 107(17):7811-7816

Lynch-Day, M. A., Klionsky, D. J. (2010). The Cvt pathway as a model for selective autophagy. FEBS Letters, 584(7):1359-1366

Mao, K., Chew, L. H., Inoue-Aono, Y., Cheong, H., Nair, U., Popelka, H. Yip, C. K., Klionsky, D. J. (2013). Atg29 phosphorylation regulates coordination of the Atg17-Atg31-Atg29 complex with the Atg11 scaffold during autophagy initiation. Proceedings of the National Academy of Science, 110(31):28752884

Mari, M., Griffith, J., Rieter, E., Krishnappa, L., Klionsky, D. J., Reggiori, F. (2010). An Atg9-containing compartment that funtions in the early steps of autophagosome biogenesis. Journal of Cell Biology, 190(6):1005-1022

Mari, M., Tooze, S. A., Reggiori, F. (2011). The puzzling origin of the autophagosomal membrane. F1000 Biology Reports, 3, 25. http://doi.org/10.3410/B3-25

Matsuoka, K., Orci, L., Amherdt, M., Bednarek, S. Y., Hamamoto, S. Schekman, R., Yeung, T. (1998). COPII-coated vesicle formation reconstituted with purified coat proteins and chemically defined liposomes. Cell, 93(2):263-275 
Matsuura, A., Tsukada, M., Wada, Y., Ohsumi, Y. (1997). Apg1p, a novel protein kinase required for the autophagic process in Saccharomyces cerevisiae. Gene, 192(2):245-250

Mizuno-Yamasaki, E., Rivera-Molina, F., Novick, P. (2012). GTPase networks in membrane traffic. Annual Review of Biochemistry, 81:637-659

Mizushima, N., Noda, T., Yoshimori, T., Tanaka, Y., Ishii, T., George, M. D., Klionsky, D. J., Ohsumi, M., Ohsumi, Y. (1998). A protein conjugation system essential for autophagy. Nature, 395(6700):395-398

Mochida, K., Oikawa, Y., Kimura, Y., Kirisako, H., Hirano, H., Ohsumi, Y., Nakatogawa, H. (2015). Receptor-mediated selective autophagy degrades the endoplasmic reticulum and the nucleus. Nature, 522(7556):359-362

Motley, A. M., Nuttall, J. M., Hettema, E. H. (2012). Pex3-anchored Atg36 tags peroxisomes for degradation in Saccharomyces cerevisiae. The EMBO Journal, 31(13): 2852-2868

Müller, J., Johnsson, N. (2008). Split-ubiquitin and the split-protein sensors: chessman for the endgame. ChemBioChem, 9(13):2029-2038

Müller, M., Schmidt, O., Angelova, M., Faserl, K., Weys, S., Kremser, L., Pfaffenwimmer, T., Dalik, T., Kraft, C., Trajanoski, Z., Lindner, H., Teis, D. (2015). The coordinated action of the MVB pathway and autophagy ensures cell survival during starvation. eLife, 4, e07736. http://doi.org/10.7554/eLife.07736

Nair, U., Yen, W-L., Mari, M., Cao, Y., Xie, Z., Baba, M., Reggiori, F., Klionsky, D. J. (2012). A role for Atg8-PE deconjugation in autophagosome formation. Autophagy, 8(5):780-793

Nakatogawa, H., Ichimura, Y., Ohsumi, Y. (2007). Atg8, a ubiquitin-like protein required for autophagosome formation, mediates membrane tethering and hemifusion. Cell, 130(1):165-178

Nakatogawa, H., Ishii, J., Asai, E., Ohsumi, Y. (2012a). Atg4 recycles inappropriately lipidated Atg8 to promote autophagosome biogenesis. Autophagy, 8(2):177-186

Nakatogawa, H., Ohbayashi, S., Sakoh-Nakatogawa, M., Kakuta, S., Suzuki, S. W., Kirisako, H., Kondo-Kakuta, C., Noda, N. N., Yamamoto, H., Ohsumi, Y. (2012b). The autophagy related protein kinase Atg1 interacts with the ubiquitin-like protein Atg8 via the Atg8 family interacting motif to facilitate autophagosome formation. Journal of Biological Chemistry, 287(34):28503-28507

Nakatogawa, H., Suzuki, K., Kamada, Y., Ohsumi, Y. (2009). Dynamics and diversity in autophagy mechanisms: lessons from yeast. Nature Reviews Molecular Cell Biology, 10(7):458-467 
Noda, N. N., Fujioka, Y., Hanada, T., Ohsumi, Y., Inagaki, F. (2013). Structure of the Atg12-Atg5 conjugate reveals a platform for stimulating Atg8-PE conjugation. EMBO Reports, 14(2):206-211

Noda, N. N., Kumeta, H., Nakatogawa, H., Satoo, K., Adachi, W., Ishii, J., Fujioka, Y., Ohsumi, Y., Inagaki, F. (2008). Structural basis of target recognition by Atg8/LC3 during selective autophagy. Genes to Cells, 13(12):1211-1218

Noda, N. N., Ohsumi, Y., Inagaki, F. (2010). Atg8-family interacting motif crucial for selective autophagy. FEBS Letters, 584(7):1379-1385

Noda, S. N., Kira, S., Yoshimori, T., Noda, T. (2013). TRAPPIII is responsible for vesicular transport from early endosomes to Golgi, facilitating Atg9 cycling in autophagy. Journal of Cell Science, 126(21):4963-4973

Noda, T., Kim, J., Huang, W. P. Baba, M., Tokunaga, C., Ohsumi, Y., Klionsky, D. J. (2000). Apg9p/Cvt7p is an integral membrane protein required for transport vesicle formation in the Cvt and autophagy pathways. Journal of Cell Biology, 148(3):465-480

Noda, T., Klionsky, D. J. (2008). The quantitative Pho8 $\Delta 60$ assay of nonspecific autophagy. Methods in Enzymology, 451:33-42

Nottingham, R. M., Pfeffer, S. R. (2009). Defining the boundaries: Rab GEFs and GAPs. Proceedings of the National Academy of Sciences USA, 106(34):14185-14186

Nuoffer, C., Davidson, H. W., Matteson, J., Meinkoth, J., Balch, W. E. (1994). A GDP-bound of rab1 inhibits protein export from the endoplasmic reticulum and transport between Golgi compartments. The Journal of Cell Biology, 125(2):225-237

Obara, K., Noda, T., Niimi, K., Ohsumi, Y. (2008). Transport of phosphatidylinositol 3-phosphate into the vacuole via autophagic membranes in Saccharomyces cerevisiae. Genes to Cells, 13(6):537-547

Obara, K., Ohsumi, Y. (2011). Ptdlns 3-kinase orchestrates autophagosome formation in yeast. Journal of Lipids, doi: 10.1155/2011/498768

Obara, K., Sekito, T., Ohsumi, Y. (2006). Assortment of phosphatidylinositol 3kinase complexes - Atg14p directs association of complex I to the preautophagosomal structure in Saccharomyces cerevisiae. Molecular Biology of the Cell, 17(4):1527-1539

Okamoto, K., Kondo-Okamoto, N., Oshumi, Y. (2009). Mitochondriaanchored receptor Atg32 mediates degradation of mitochondria via selective autophagy. Developmental Cell, 17(1):87-97

Obara, K., Sekito, T., Ohsumi, Y. (2006). Assortment of phosphatidylinositol 3kinase complexes-Atg14p directs association of complex I to the pre- 
autophagosomal structure in Saccharomyces cerevisiae. Molecular Biology of the Cell, 17(4):1527-1539

Pan, X., Eathiraj, S., Munson, M., Lambright, D. G. (2006). TBC-domain GAPs for Rab GTPases accelerate GTP hydrolysis by a dual-finger mechanism. Nature, 442:303-306

Papinski, D., Schuschnig, M., Reiter, W., Wilhelm, L., Barnes, C. A., Maiolica, A., Hansmann, I., Pfaffenwimmer, T., Kijanska, M., Stoffel, I., Lee, S. S., Brezovich, A., Lou, J. H., Turk, B. E., Aebersold, R., Ammerer, G., Peter, M., Kraft, C. (2014). Early steps in autophagy depend on direct phosphorylation of Atg9 by the Atg1 kinase. Molecular Cell, 53(3):471-483

Pengo, N., Agrotis, A., Prak, K., Jones, J., Ketteler, R. (2017). A reversible phospho-switch mediated by ULK1 regulates the activity of autophagy protease ATG4B. Nature Communications, 8(1):294 doi:10.1038/s41467017-00303-2

Pereira-Leal, J. B., Seabra, M. C. (2001). Evolution of the Rab family of small GTP-binding proteins. Journal of Molecular Biology, 313(4):889-901

Pfanner, N., Müller, H. K., Harmey, M. A. Neupert, W. (1987). Mitochondrial protein import: involvement of the mature part of a cleavable precursor protein in the binding to receptor sites. The EMBO Journal, 6(11):34493454

Pfeffer, S. R. (2013). Rab GTPase regulation of membrane identity. Current Opinion in Cell Biology, 25(4):414-419

Pfeffer, S. R. (2017). Rab GTPases: master regulators that establish the secretory and endocytic pathways. Molecular Biology of the Cell, 28(6): 712-715

Pind, S. N., Nuoffer, C., McCaffery, M., Plutner, H., Davidson, H. W., Farquar, M. G., Balch, W.E. (1994). Rab1 and Ca2+ are required for the fusion of carrier vesicles mediating endoplasmic reticulum to Golgi transport. The Journal of Cell Biology, 125(2):239-252

Plutner, H., Cox, A. D., Pind, S., Khosravi-Far, R., Bourne, J. R., Schwaninger, R., Der, C. J., Balch, W. E. (1991). Rab1b regulates vesicular transport between the endoplasmic reticulum and successive Golgi compartments. The Journal of Cell Biology, 115(1):31-43.

Popelka, H., Klionsky, D. J. (2015). Analysis of the native conformation of the LIR/AIM motif in the Atg8/LC3/GABARAP-binding proteins. Autophagy, 11(12):2153-2159

Popovic, D., Akutsu, M., Novak, I., Harper, J. W., Behrends, C., Dikic, I. (2012). Rab GTPase-activating proteins in autophagy: regulation of endocytic and autophagy pathways by direct binding to human ATG8 modifiers. Molecular and Cellular Biology, 32(9):1733-1744 
Pylypenko, O., Rak, A., Durek, T., Kushnir, S., Dursina, B. E., Thomae, N. H., Constantinescu, A. T., Brunsveld, L., Watzke, A., Waldmann, H., Goody, R. S., Alexandrov, K. (2006). Structure of doubly prenylated Ypt1: GDI complex and the mechanism of GDI-mediated Rab recycling. The EMBO Journal, 25(1):13-23

Pylypenko, O., Hammich, H., Yu, I.-M., Houdusse, A. (2017). Rab GTPases and their interacting protein partners: structural insights into Rab functional diversity. Small GTPases, 0(0):1-27 doi: 10.1080/21541248.2017.1336191

Ragusa, M. J., Stanley, R. E., Hurley, J. H. (2012). Architecture of the Atg17 complex as a scaffold for autophagosome biogenesis. Cell, 151(7):15011512

Rak, A., Ferodov, R., Alexandrov, K., Albert, S., Goody, R. S., Gallwitz, D., Scheidig, A. J. (2000). Crystal structure of the GAP domain of Gyp1p: first insights into interaction with Ypt/Rab proteins. The EMBO Journal, 19(19):5105-5113

Rao, Y., Perna, M. G., Hofmann, B., Beier, V., Wollert, T. (2016). The Atg1kinase complex tethers Atg9-vesicles to initiate autophagy. Nature Communications, 7:10338. doi:10.1038/ncomms10338

Reggiori, F., Klionsky, D. J. (2013). Autophagic processes in yeast: mechanism, machinery and regulation. Genetics, 194(2):341-361

Reggiori, F., Tucker, K. A., Stromhaug, P. E., Klionsky, D. J. (2004). The Atg1Atg13 complex regulates Atg9 and Atg23 retrieval transport from the preautophagosomal structure. Developmetal Cell, 6(1):79-90

Rivera-Molina, F. E., Novick, P. J. (2009). A Rab GAP cascade defines the boundary between two Rab GTPases on the secretory pathway.

Proceedings of the National Academy of Sciences USA, 106(34):1440814413

Rogov, V., Dötsch, V., Johansen, T., Kirkin, V. (2014). Interactions between autophagy receptors and ubiquitin-like proteins form the molecular basis for selective autophagy. Molecular Cell, 53(2):167-178

Ryter, S. W., Cloonan, S. M., Choi, A. M. K. (2013). Autophagy: a critical regulator of cellular metabolism and homeostasis. Molecules and Cells, 36(1):7-16

Sacher, M., Jiang, Y., Barrowman, J., Scarpa, A., Burston, J., Zhang, L., Schieltz, D., Yates, J. R., Abeliovich, H., Ferro-Novick, S. (1998). TRAPP, a highly conserved novel complex on the cis-Golgi that mediates vesicle docking and fusion. The EMBO Journal, 17(9):2494-2503. http://doi.org/10.1093/emboj/17.9.2494 
Sánchez-Wandelmer, J., Kriegenburg, F., Rohringer, S., Schuschnig, M., Gómez-Sánchez, R., Zens, B., Abreu, S., Hardenberg, R., Hollenstein, D., Gao, J., Ungermann, C., Martens, S., Kraft, C., Reggiori, F. (2017). Atg4 proteolytic activity can be inhibited by Atg1 phosphorylation. Nature Communications, 8(1):295. http://doi.org/10.1038/s41467-017-00302-3

Sanchez-Wandelmer, J., Ktistakis, N. T., Reggiori, F. (2015). ERES: sites for autophagosome biogenesis and maturation? Journal of Cell Science, 128(2):185-192

Saraste, J., Lahtinen, U., Goud, B. (1995). Localization of the small GTP-binding protein rab1p to early compartments of the secretory pathway. Journal of Cell Science, 108:1541-1552

Sawa-Makarska, J., Abert, C., Romanov, J., Zens, B., Ibiricu, I., Martens, S. (2014). Cargo binding to Atg19 unmasks further Atg8 binding sites to mediate membrane-cargo apposition during selective autophagy. Nature Cell Biology, 16(5):425-433

Sclafani, A., Chen, S., Rivera-Molina, F., Reinisch, K., Novick, P., FerroNovick, S. (2010). Establishing a role for the GTPase Ypt1p at the late golgi. Traffic, 11(4):520-532

Scherens, B., Goffeau, A. (2004). The uses of genome-wide yeast mutant collections. Genome Biology, 5(7):229. http://doi.org/10.1186/gb-2004-5-7229

Schwarz, D. S., Blower, M. D. (2016). The endoplasmic reticulum: structure, function and response to cellular signaling. Cellular and Molecular Life Sciences, 73:79-94

Scott, S. V., Guan, J., Hutchins, M. U., Kim, J., Klionsky, D. J. (2001). Cvt19 is a receptor for the cytoplasm-to-vacuole targeting pathway. Molecular Cell 7(6):1131-1141

Scott, S.V., Hefner-Gravink, A., Morano, K. A., Noda, T., Ohsumi, Y., Klionsky, D. J. (1996). Cytoplasm-to-vacuole targeting and autophagy employ the same machinery to deliver proteins to the yeast vacuole. Proceedings of the National Academy of Science USA, 93(22):12304-12308

Seabra, M. C., Mules, E. H., Hume, A. N. (2002). Rab GTPases, intracellular traffic and disease. Trends in Molecular Medicine, 8(1):23-30

Segev, N. (1991). Mediation of the attachment or fusion step in vesicular transport by the GTP-binding Ypt1 protein. Science, 252(5012):1553-1556

Segev, N. (2001). Ypt/Rab GTPases: regulators of protein trafficking. Science Signaling, 100:re11 DOI: 10.1126/stke.2001.100.re11

Shapshak, P., Duncan, R., Kangueane, P., Somboonwit, C., Sinnott, J., Commins, D., Singer, E., Levine, A. (2011). HIV associated dementia and 
HIV encephalitis II: genes on chromosome 22 expressed in individually microdissected Globus pallidus neurons (Preliminary analysis).

Bioinformation, 6(5):183-186

Shintani, T., Huang W.-P., Stromhaug, P. E., Klionsky, D. J.

(2002). Mechanism of cargo selection in the cytoplasm to vacuole targeting pathway. Developmental Cell, 3(6):825-837

Shpilka, T., Weidberg, H., Pietrokovski, S., Elazar, Z. (2011). Atg8: an autophagy-related ubiquitin-like protein family. Genome Biology, 12(7):226 http://doi.org/10.1186/gb-2011-12-7-226

Sikorski, R. S., Hieter, P. (1989). A system of shuttle vectors and yeast host strains designed for efficient manipulation of DNA in Saccharomyces Cerevisiae. Genetics, 122(1):19-27

Skytte Rasmussen, M., Mouilleron, S., Kumar Shrestha, B., Wirth, M., Lee, R., Bowitz Larsen, K., Princely, Y. A., O'Reilly, N., Sjøttem, E., Tooze, S. A., Lamark, T., Johansen, T. (2017). ATG4B contains a C-terminal LIR motif important for binding and efficient cleavage of ATG8 family proteins. Autophagy, 13(5):834-853

Stehling, O., Lill, R. (2013). The role of mitochondria in cellular iron-sulfur protein biogenesis: mechanisms, connected processes, and diseases. Cold Spring Harbor Perspectives in Biology, 5(8): a011312 doi:

10.1101/cshperspect.a011312

Stolz, A., Ernst, A., Dikic, I. (2014). Cargo recognition and trafficking in selective autophagy. Nature Cell Biology, 16(6):495-501

Stjepanovic, G., Davies, C. W., Stanley, R. E., Ragusa, M. J., Kim, D. J., Hurley, J. H. (2014). Assembly and dynamics of the autophagy-initiating Atg1 complex. Proceedings of the National Academy of Sciences USA, 111(35):12793-12798

Shintani, T., Suzuki, K., Kamada, Y., Noda, T., Ohsumi, Y. (2001). Apg2p functions in autophagosome formation on the perivacuolar structure. Journal of Biological Chemistry, 276(32):30452-30460

Shintani, T., W.-P. Huang, Stromhaug, P. E., Klionsky, D. J. (2002). Mechanism of cargo selection in the cytoplasm to vacuole targeting pathway. Developmental Cell, 3(6):825-837

Slobodkin, M. R., Elazar, Z. (2013). The Atg8 family: multifunctional ubiquitin-like key regulators of autophagy. Essays in Biochemistry, 55:51-64 doi: 10.1042/bse0550051

Stanley, R. E., Ragusa, M. J., Hurley, J. H. (2014). The beginning of the end: how scaffolds nucleate autophagosome biogenesis. Trends in Cell Biology, 24(1):73-81 
Stenmark, H. (2009). Rab GTPases as coordinators of vesicle traffic. Nature Reviews Molecular Cell Biology, 10(8):513-525

Stephan, J. S., Yeh, Y.-Y., Ramachandran, V., Deminoff, S. J., Herman, P. K. (2009). The Tor and PKA signaling pathways independently target the Atg1/Atg13 protein kinase complex to control autophagy. Proceedings of the National Academy of Sciences USA, 106(40):17049-17054

Suzuki, H., Osawa, T., Fujioka, Y., Noda, N. N. (2017). Structural biology of the core autophagy machinery. Current Opinion in Structural Biology, 43:10-17 doi: 10.1016/j.sbi.2016.09.010.

Suzuki, K., Kirisako, T., Kamada, Y., Mizushima, N., Noda, T., Ohsumi, Y. (2001). The pre-autophagosomal structure organized by concerted functions of APG genes is essential for autophagosome formation. The EMBO Journal, 20(21):5971-5981

Suzuki, K., Kubota, Y., Sekito, T., Ohsumi, Y. (2007). Hierarchy of Atg proteins in pre-autophagosomal structure organization. Genes to Cells, 12(2):209218

Suzuki, K., Ohsumi, Y. (2010). Current knowledge of the pre-autophagosomal structure (PAS). FEBS Letters, 584(7):1280-1286

Suzuki, S. W., Yamamoto, H., Oikawa, Y., Kondo-Kakuta, C., Kimura, Y., Hirano, H., Ohsumi, Y. (2015). Atg13 HORMA domain recruits Atg9 vesicles during autophagosome formation. Proceedings of the National Academy of Science USA, 112(11):3350-3355

Szatmári, Z., Sass, M. (2014). The autophagic roles of Rab small GTPases and their upstream regulators: a review. Autophagy, 10(7):1154-1166

Taherbhoy, A. M., Kaiser, S. E., Williams, A. H., Deng, A., Nourse, A., Hammel, M., Kurinov, I., Rock, C. O., Green, D. R., Schulman, B. A. (2011). Atg8 transfer from Atg7 to Atg3: a distinctive E1-E2 architecture and mechanism in the autophagy pathway. Molecular Cell, 44(3):451-461

Tan, D., Cai, Y., Wang, J., Zhang, J., Menon, S., Chou, H.-T., Ferro-Novick, S., Reinisch, K. M., Walz, T. (2013). The EM structure of the TRAPPIII complex leads to the identification of a requirement for COPII vesicles on the macroautophagy pathway. Proceedings of the National Academy of Science USA, 110(48):19432-19437

Teichert, U., Mechler, B., Müller, H., Wolf, D. H. (1989). Lysosomal (vacuolar) proteinases of yeast are essential catalysts for protein degradation, differentiation, and cell survival. Journal of Biological Chemistry, 264(27):16037-16045

Tempel, W., Tong, Y., Dimov, S., Bochkarev, A., Park, H. (2008). First crystallographic models of human TBC domains in the context of a familywide structural analysis. Proteins, 71(1):497-502 
Tenreiro, S., Franssens, V., Winderickx, J., Outeiro, T. F. (2017). Yeast models of Parkinson's disease-associated molecular pathologies. Current Opinion in Genetics and Development, 44:73-83

Thomas, L. L., Joiner, A. M. N., Fromme, J. C. (2017). The TRAPPIII complex activates the GTPase Ypt1 (Rab1) in the secretory pathway. The Journal of Cell Biology, DOI: 10.1083/jcb.201705214

Tooze, S. A. (2013). Current views on the source of the autophagosomal membrane. Essays in Biochemistry, 55:29-38

Urbé, S. (2005). Ubiquitin and endocytic protein sorting. Essays in Biochemistry, $41: 81-98$

Vézina, C., Kudelski, A., Sehgal, S. N. (1975). Rapamycin (AY-22,989), a new antifungal antibiotic. I. Taxonomy of the producing streptomycete and isolation of the active principle. The Journal of Antibiotics, 28(10):721-726.

Wandinger-Ness, A., Zerial, M. (2014). Rab proteins and the compartmentalization of the endosomal system. Cold Spring Harbor Perspectives in Biology, 6(11):a022616. doi:10.1101/cshperspect.a022616

Wach, A., Brachat, A., Alberti-Segui, C., Rebischung, C., Philippsen, P. (1997). Heterologous HIS3 marker and GFP reporter modules for PCRtargeting in Saccharomyces cerevisiae. Yeast, 13(11):1065-1075

Wang, J., Davis, S., Zhu, M., Miller, E. A., Ferro-Novick, S. (2017).

Autophagosome formation: where the secretory and autophagy pathways meet. Autophagy, 13(5):973-974

Wang, J., Menon, S., Yamasaki, A., Chou, H.-T., Walz, T., Jiang, Y., FerroNovick, S. (2013). Ypt1 recruits the Atg1 kinase to the preautophagosomal structure. Proceedings of the National Academy of Science USA, 110(24):24 9800-9805 doi: 10.1073/pnas.1302337110.

Weidberg, H., Shpilka, T., Shvets, E., Abada, A., Shimron, F., Elazar, Z. (2011). LC3 and GATE-16N termini mediate membrane fusion processes required for autophagosome biogenesis. Developmental Cell, 20(4):444454

Welter, E., Montino, M., Reinhold, R., Schlotterhose, P., Krick, R., Dudek, J., Rehling, P., Thumm, M. (2013). Uth1 is a mitochondrial inner membrane protein dispensable for post-log-phase and rapamycin-induced mitophagy. The FEBS Journal, 280(20):4970-4982

Wen, X., Klionsky, D. J. (2016). An overview of macroautophagy in yeast. Journal of Molecular Biology, 428(9):1681-1699

Winzeler, E. A, Shoemaker, D. D., Astromoff, A., Liang, H., Anderson, K., Andre, B., Bangham, R., Benito, R., Boeke, J. D., Bussey, H., et al. 
(1999). Functional characterization of the $S$. cerevisiae genome by gene deletion and parallel analysis. Science, 85(5429):901-906

Welter, E., Thumm, M., Krick, R. (2010). Quantification of nonselective bulk autophagy in S. cerevisiae using Pgk1-GFP. Autophagy, 6(6):794-797

Wu, H., Wei, H., Sehgal, S. A., Liu, L., Chen, Q. (2016). Mitophagy receptors sense stress signals and couple mitochondrial dynamic machinery for mitochondrial quality control. Free Radical Biology and Medicine, 100:199209

Xie, Z., Nair, U., Klionsky, D. J. (2008). Atg8 controls phagophore expansion during autophagosome formation. Molecular Biology of the Cell, 19(8): 3290-3298

Yamano, K., Fogel, A. I., Wang, C., van der Bliek, A. M., Youle, R. J. (2014). Mitochondrial Rab GAPs govern autophagosome biogenesis during mitophagy. eLife, 3, e01612. http://doi.org/10.7554/eLife.01612

Yamaguchi, M., Noda, N. N., Nakatogawa, H., Kumeta, H., Ohsumi, Y., Inagaki, F. (2010). Autophagy-related protein 8 (Atg8) family interacting motif in Atg3 mediates the Atg3-Atg8 interaction and is crucial for the cytoplasm-to-vacuole targeting pathway. Journal of Biological Chemistry, 285(38):29599-29607

Yamaguchi, O., Murakawa, T., Nishida, K., Otsu, K. (2016). Receptor-mediated mitophagy. Journal of Molecular and Cellular Cardiology, 95:50-56 doi: 10.1016/j.yjmcc.2016.03.010. Epub 2016 Mar 25

Yamamoto, H., Kakuta, S., Watanabe, T. M., Kitamura, A., Sekito, T., KondoKakuta, C., Ichikawa R., Kinjo, M., Ohsumi, Y. (2012). Atg9 vesicles are an important membrane source during early steps of autophagosome formation. Journal of Cell Biology, 198(2):219-233

Yamasaki, A., Noda, N. N. (2017). Structural biology of the Cvt pathway. Journal of Molecular Biology, 429(4):531-542

Yang, S., Rosenwald, A. G. (2016). Autophagy in Saccharomyces cerevisiae requires the monomeric GTP-binding proteins, Arl1 and Ypt6. Autophagy, 12(10):1721-1737

Yao, Z., Delorme-Axford, E., Backues, S. K., Klionsky, D. J. (2015). Atg41/lcy2 regulates autophagosome formation. Autophagy, 11(12):2288-2299

Yen, W-L., Klionsky, D. J. (2008). How to live long and prosper: autophagy, mitochondria and aging. Physiology, 23(5):248-262

Ylä-Anttila, P., Vihinen, H., Jokitalo, E., Eskelinen, E. L. (2009). 3D tomography reveals connections between the phagophore and endoplasmic reticulum. Autophagy, 5(8):1180-1185. 
Yorimitsu, T., Klionsky, D. J. (2005). Atg11 links cargo to the vesicle-forming machinery in the cytoplasm to vacuole targeting pathway. Molecular Biology of the Cell, 16(4):1593-1605

Zaffagnini, G., Martens, S. (2016). Mechanisms of selective autophagy. Journal of Molecular Biology, 428(9):1714-1724

Zhen, Y., Stenmark, H. (2015). Cellular functions of Rab GTPases at a glance. Journal of Cell Science, 128(17):3171-3176

Zoppino, F. C. M., Militello, R. D., Slavin, I., Alvarez, C., Colombo, M. I. (2010). Autophagosome formation depends on the small GTPase Rab1 and functional ER exit sites. Traffic, 11(9):1246-1261 


\section{Curriculum vitae}

\begin{tabular}{|c|c|}
\hline Personal Data & \\
\hline $\begin{array}{l}\text { Name } \\
\text { Born }\end{array}$ & $\begin{array}{l}\text { Anne Lisa Mitter } \\
\text { 27.07.1988 in Hanover, Germany }\end{array}$ \\
\hline Education & \\
\hline 06/2014 - present & $\begin{array}{l}\text { Dissertation at the Georg-August University of } \\
\text { Goettingen } \\
\text { Department for Biochemistry, } \\
\text { Center for Biochemistry and Molecular Cell Biology } \\
\text { Prof. Dr. Michael Thumm, } \\
\text { Dr. Roswitha Krick }\end{array}$ \\
\hline 10/2011 - 03/2014 & $\begin{array}{l}\text { Master Molecular Life Science, Friedrich Schiller } \\
\text { University Jena } \\
\text { Major subjects: molecular cell biology, molecular } \\
\text { genetics and molecular developmental biology } \\
\text { Master thesis: Hans-Knöll Institue, Jena } \\
\text { Department for Cell- and Molecularbiology, } \\
\text { Prof. Dr. Hans Peter Saluz } \\
\text { Thesis title: Four dimensional molecular imaging of } \\
\text { insulin-induced bone disturbances in chick embryos } \\
\text { via Positron Emission Tomography and Computed } \\
\text { Tomography }\end{array}$ \\
\hline $10 / 2008-10 / 2011$ & $\begin{array}{l}\text { Bachelor Biology, Leibnitz University Hanover } \\
\text { Bachelor thesis: University of veterinary medicine, } \\
\text { Hanover } \\
\text { Division of ecology and evolution } \\
\text { PD Dr. Heike Hadrys } \\
\text { Thesis title: Population genetic research on a } \\
\text { potential bioindicatior for climate change: Orthetrum } \\
\text { coerulescens (FABRICIUS, 1798) }\end{array}$ \\
\hline 08/2005 - 06/2008 & $\begin{array}{l}\text { Gymnasium: Berufliches Gymnasium Wirtschaft, } \\
\text { Springe }\end{array}$ \\
\hline
\end{tabular}

